\title{
Enantioselective Construction of Spiro Quaternary Carbon Stereocenters via Pd-Catalyzed Intramolecular $\alpha$-Arylation
}

Ting Wu, Xuehua Kang, Heng Bai, Wenrui Xiong, Guangqing Xu, Wenjun Tang ${ }^{*, a, b}$

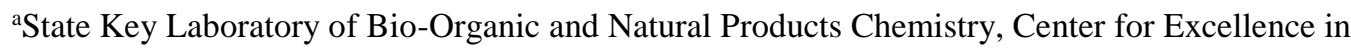
Molecular Synthesis, Shanghai Institute of Organic Chemistry, University of Chinese Academy of Sciences, 345 Ling Ling Rd, Shanghai 200032, China

${ }^{b}$ School of Chemistry and Material Sciences, Hangzhou Institute for Advanced Study, University of Chinese Academy of Sciences, 1 Sub-lane Xiangshan, Hangzhou 310024, China

tangwenjun@sioc.ac.cn

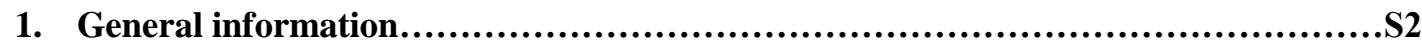

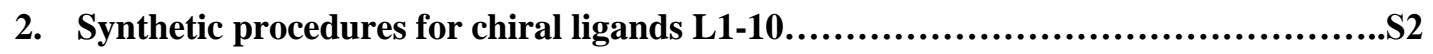

3. Preparation of substrates for Pd-catalyzed asymmetric intramolecular $\alpha$-arylation

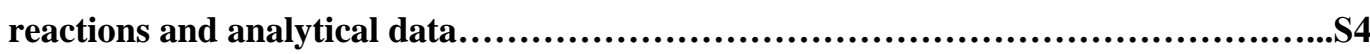

4. General procedures of Pd-catalyzed asymmetric intramolecular $\alpha$-arylation reactions.

5. Analytical data of the products of Pd-catalyzed asymmetric intramolecular $\alpha$-arylation reactions.

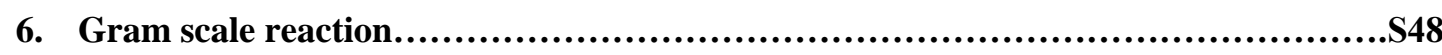

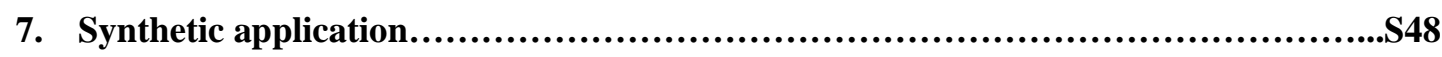

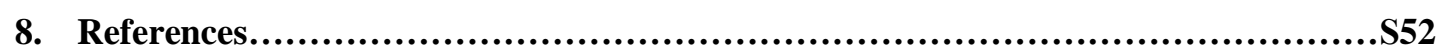

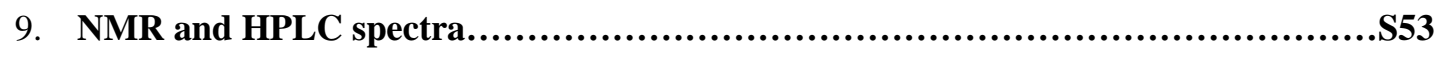

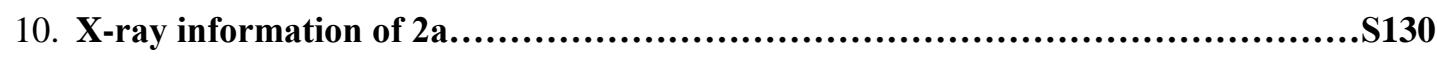




\section{General information}

Reactions were set-up on the bench top and carried out under nitrogen atmosphere in Schlenk tubes unless otherwise noted. All reagents were used as received from commercial sources, unless otherwise specified, or prepared as described in the literature. Solvent was purified according to the procedure from "Purification of Laboratory Chemicals".

Analytical thin layer chromatography (TLC) was performed on precoated silica gel 60 GF254 plates. Flash column chromatography was performed using Tsingdao silica gel (200-300 mesh). Visualization on TLC was achieved by use of UV light $(254 \mathrm{~nm})$, iodine or basic KMnO4 dip. ${ }^{31} \mathrm{P}$ NMR, ${ }^{19} \mathrm{~F}$ NMR and ${ }^{13} \mathrm{C}$ NMR data were recorded on a Bruker-Ultrashield PLUS 400, a $500 \mathrm{MHz}$ Agilent or a Bruker Avance $600 \mathrm{AV}$ spectrometer with $\mathrm{CDCl}_{3}$ as the solvent. ${ }^{1} \mathrm{H}$ shifts were referenced to $\mathrm{CDCl}_{3}$ at $7.26 \mathrm{ppm}$. ${ }^{13} \mathrm{C}$ shifts were referenced to $\mathrm{CDCl}_{3}$ at $77.16 \mathrm{ppm}$ and obtained with ${ }^{1} \mathrm{H}$ decoupling. ${ }^{31} \mathrm{P}$ shifts were referenced to $85 \% \mathrm{H}_{3} \mathrm{PO}_{4}$ in $\mathrm{D}_{2} \mathrm{O}$ at $0.0 \mathrm{ppm}$ as external standard and obtained with ${ }^{1} \mathrm{H}$ decoupling. ${ }^{19} \mathrm{~F}-\mathrm{NMR}$ spectra were recorded with complete proton decoupling using $\mathrm{BF}_{3} \cdot \mathrm{Et}_{2} \mathrm{O}$ (-153 ppm for ${ }^{19} \mathrm{~F}$ NMR spectra) as an external standard. Multiplicities are abbreviated as follows: singlet (s), doublet (d), triplet ( $\mathrm{t}$ ), quartet (q), doubletdoublet (dd), multiplet (m), and broad (br). MS was measured on Agilent 1100 Series LC/MSD mass spectrometer, or Shimadzu LCMS-2010EV (EI) or Brukerdaltonics APEX III (HR-EI) mass spectrometers or Bruker maXis 4G TOF mass spectrometers. All reagents were used as received

from commercial sources, unless otherwise specified, or prepared as described in the literature. All reagents were weighed and handled in air and refilled with an inert atmosphere of nitrogen, unless otherwise specified. All solvents were dried and distilled prior to use by usual laboratory methods. Anhydrous THF were distilled from sodium/benzophenone immediately prior to use. Heating of experiments was conducted on an oil bath. Abbreviations are as follows: dba, dibenzylideneacetone; LDA, lithium diisopropylamide; TEA, triethylamine; DCM, dichloromethane; EA, ethyl acetate; PE, petroleum ether.

\section{Synthetic procedures for ligands L1-10}

Ligands L1-7 and L10 were prepared according to procedures described in our previous reports $^{[1-4]}$. 


\section{Synthesis of L8:}

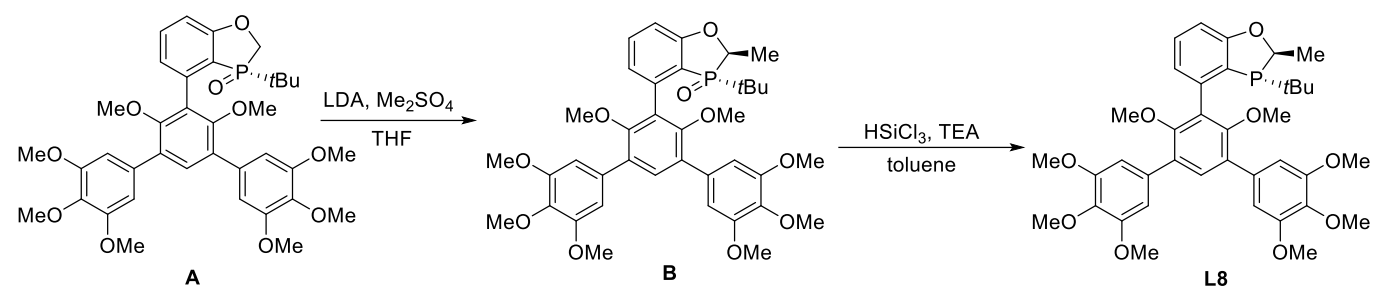

The synthesis of compound $\mathbf{A}$ was followed according to procedures described in previous reports ${ }^{4}$.

To a solution of compound $\mathbf{A}(0.8 \mathrm{~g}, 1.18 \mathrm{mmol}, 1$ equiv $)$ in THF $(9 \mathrm{~mL})$ at $-78{ }^{\circ} \mathrm{C}$ was added LDA $(1.36 \mathrm{~mL}, 2.0 \mathrm{M}$ in heptane/THF, $2.71 \mathrm{mmol}, 2.3$ equiv) over $5 \mathrm{~min}$. The resulting mixture was stirred at $-78^{\circ} \mathrm{C}$ for $1 \mathrm{~h}$ followed by the addition of $\mathrm{Me}_{2} \mathrm{SO}_{4}(0.26 \mathrm{~mL}, 2.71 \mathrm{mmol}, 2.3$ equiv). After stirring at $-78{ }^{\circ} \mathrm{C}$ for $1 \mathrm{~h}$, the mixture was allowed to warm to $\mathrm{rt}$ over $1 \mathrm{~h}$ and kept stirring at $\mathrm{rt}$ overnight. Saturated aqueous $\mathrm{NH}_{4} \mathrm{Cl}(20 \mathrm{~mL})$ and ethyl acetate $(20 \mathrm{~mL})$ were added. The organic layer was separated and the aqueous layer was further extracted with ethyl acetate $(20 \mathrm{~mL})$. The combined organic layers was washed with brine, dried over $\mathrm{Na}_{2} \mathrm{SO}_{4}$, concentrated, and purified by silica gel column chromatography (eluent: petroleum ether/EtOAc=1/1) to give product $\mathbf{B}(680 \mathrm{mg}$, $83 \%)$.

To a solution of compound $\mathbf{B}(90 \mathrm{mg}, 0.13 \mathrm{mmol}, 1$ equiv) in toluene $(4 \mathrm{~mL})$ at $\mathrm{rt}$ was added TEA ( $0.11 \mathrm{~mL}, 0.78 \mathrm{mmol}, 6$ equiv) and $\mathrm{HSiCl}_{3}(0.066 \mathrm{ml}, 0.65 \mathrm{mmol}, 5$ equiv). The mixture was stirred at $80{ }^{\circ} \mathrm{C}$ (oiling bath) for $12 \mathrm{~h}$, and then cooled to room temperature. To the mixture was added carefully $30 \%$ aqueous $\mathrm{NaOH}$ solution $(2.5 \mathrm{~mL})$. Gas was generated during the addition. The resulting mixture was further stirred at $80{ }^{\circ} \mathrm{C}$ for $0.5 \mathrm{~h}$. When the mixture was cooled to room temperature, ethyl acetate $(4 \mathrm{~mL})$ was added. The organic layer was separated and the aqueous layer was further washed with ethyl acetate for several times. The combined organic layer was washed with brine, dried over $\mathrm{Na}_{2} \mathrm{SO}_{4}$, concentrated, and purified by silica gel column chromatography (eluent: petroleum ether/EtOAc=5/1) to afford the desired product $\mathbf{L 8}(50 \mathrm{mg}, 0.46 \mathrm{mmol}, 57 \%)$ as white solid. L8: $[\alpha]_{\mathrm{D}}{ }^{25}=-46.3\left(\mathrm{c}=1.0, \mathrm{CHCl}_{3}\right) .{ }^{1} \mathrm{H} \mathrm{NMR}\left(500 \mathrm{MHz}, \mathrm{CDCl}_{3}\right) \delta 7.39-7.35(\mathrm{~m}, 1 \mathrm{H})$, $7.34(\mathrm{~s}, 1 \mathrm{H}), 6.96-6.91(\mathrm{~m}, 2 \mathrm{H}), 6.84(\mathrm{~s}, 2 \mathrm{H}), 6.78(\mathrm{~s}, 2 \mathrm{H}), 5.06(\mathrm{q}, J=7.0 \mathrm{~Hz}, 1 \mathrm{H}), 3.90(\mathrm{~d}, J=$ $0.6 \mathrm{~Hz}, 6 \mathrm{H}), 3.88$ (s, 6H), 3.86 (s, 6H), 3.40 (s, 3H), 3.36 (s, 3H), 1.42 (dd, $J=16.3,7.1 \mathrm{~Hz}, 3 \mathrm{H}$ ), $0.92(\mathrm{~d}, J=12.0 \mathrm{~Hz}, 9 \mathrm{H}) .{ }^{13} \mathrm{C} \mathrm{NMR}\left(126 \mathrm{MHz}, \mathrm{CDCl}_{3}\right) \delta 162.9,155.2,154.7,153.1(\mathrm{~d}, J=16.7$ $\mathrm{Hz}), 140.0(\mathrm{~d}, J=17.3 \mathrm{~Hz}), 137.1,134.3,134.1,132.1,130.5,130.1,129.5,123.7$ (d, $J=17.2 \mathrm{~Hz})$, 122.7, 110.3, 106.2, 105.7, 79.1, 79.0, 61.1, 60.9 (d, $J=4.7 \mathrm{~Hz}), 56.1$ (d, $J=11.5 \mathrm{~Hz}), 31.1(\mathrm{~d}, J=$ 
$18.9 \mathrm{~Hz}), 27.0(\mathrm{~d}, J=14.4 \mathrm{~Hz}), 21.5,21.3 .{ }^{31} \mathrm{P} \mathrm{NMR}\left(162 \mathrm{MHz}, \mathrm{CDCl}_{3}\right) \delta 8.21(\mathrm{~s})$. HRMS (ESI) calculated for $\left[\mathrm{M}+\mathrm{H}, \mathrm{C}_{38} \mathrm{H}_{46} \mathrm{O}_{9} \mathrm{P}\right]^{+}: 677.2874$; found: 677.2867. Melting point: $194{ }^{\circ} \mathrm{C}$.

\section{Synthesis of L9:}
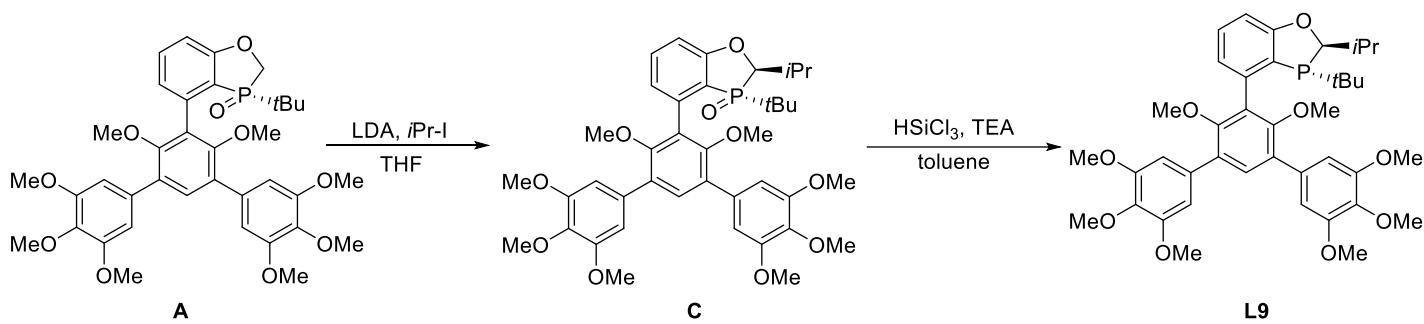

To a solution of compound $\mathbf{A}\left(200 \mathrm{mg}, 0.3 \mathrm{mmol}, 1\right.$ equiv.) in THF $(3 \mathrm{~mL})$ at $-78{ }^{\circ} \mathrm{C}$ was added LDA ( $0.44 \mathrm{~mL}, 2.0 \mathrm{M}$ in heptane/THF, $0.88 \mathrm{mmol}, 3$ equiv.) over $5 \mathrm{~min}$. The resulting mixture was stirred at $-78^{\circ} \mathrm{C}$ for $1 \mathrm{~h}$ followed by the addition of $i \operatorname{PrI}(0.09 \mathrm{~mL}, 0.88 \mathrm{mmol}, 3$ equiv.). The mixture was allowed to warm to rt over $1 \mathrm{~h}$ and kept stirring at rt overnight. Saturated aqueous $\mathrm{NH}_{4} \mathrm{Cl}$ (20 $\mathrm{mL})$ and ethyl acetate $(20 \mathrm{~mL})$ were added. The organic layer was separated and the aqueous layer was further extracted with ethyl acetate $(20 \mathrm{~mL})$. The combined organic layers was washed with brine, dried over $\mathrm{Na}_{2} \mathrm{SO}_{4}$, concentrated, and purified by silica gel column chromatography (eluent: petroleum ether/EtOAc=1/1) to give product $\mathbf{C}(126 \mathrm{mg}, 60 \%$ yield $)$.

To a solution of compound $\mathbf{C}(110 \mathrm{mg}, 0.146 \mathrm{mmol}$, lequiv) in toluene ( $4 \mathrm{~mL})$ at $\mathrm{rt}$ was added TEA (0.2 ml, $1.46 \mathrm{mmol}, 10$ equiv) and $\mathrm{HSiCl}_{3}(0.08 \mathrm{~mL}, 0.73 \mathrm{mmol}, 5$ equiv). The mixture was stirred at $80^{\circ} \mathrm{C}$ for $12 \mathrm{~h}$, and then cooled to room temperature. To the mixture was added carefully $30 \%$ aqueous $\mathrm{NaOH}$ solution $(2 \mathrm{~mL})$. Gas was generated during addition. The resulting mixture was further stirred at $80{ }^{\circ} \mathrm{C}$ (oiling bath) for $0.5 \mathrm{~h}$. When the mixture was cooled to rt, ethyl acetate (4 $\mathrm{mL}$ ) was added. The organic layer was separated and the aqueous layer was further washed with ethyl acetate for several times. The combined organic layer was washed with brine, dried over $\mathrm{Na}_{2} \mathrm{SO}_{4}$, concentrated, and purified by silica gel column chromatography (eluent: petroleum ether/EtOAc $=5 / 1)$ to afford the desired product $\mathbf{L 9}(80 \mathrm{mg}, 78 \%)$ as white solid. $\mathbf{L 9}:[\alpha]_{\mathrm{D}}^{25}=-44.1$ $\left(\mathrm{c}=1.0, \mathrm{CHCl}_{3}\right) .{ }^{1} \mathrm{H}$ NMR $\left(500 \mathrm{MHz}, \mathrm{CDCl}_{3}\right) \delta 7.34(\mathrm{dd}, J=9.1,6.5 \mathrm{~Hz}, 2 \mathrm{H}), 6.94-6.89(\mathrm{~m}, 4 \mathrm{H})$, $6.78(\mathrm{~s}, 2 \mathrm{H}), 4.66(\mathrm{~d}, J=6.5 \mathrm{~Hz}, 1 \mathrm{H}), 3.89$ (d, $J=3.3 \mathrm{~Hz}, 12 \mathrm{H}), 3.86$ (s, 6H), $3.40(\mathrm{~s}, 3 \mathrm{H}), 3.24$ (s, $3 \mathrm{H}), 1.01(\mathrm{~d}, J=6.7 \mathrm{~Hz}, 3 \mathrm{H}), 0.96(\mathrm{~d}, J=6.7 \mathrm{~Hz}, 3 \mathrm{H}), 0.88(\mathrm{~d}, J=12.0 \mathrm{~Hz}, 9 \mathrm{H}) .{ }^{13} \mathrm{C}$ NMR $(126$ $\left.\mathrm{MHz}, \mathrm{CDCl}_{3}\right) \delta 164.2,155.2,154.8,153.1,152.9,139.1(\mathrm{~d}, J=17.7 \mathrm{~Hz}), 137.1(\mathrm{~d}, J=8.6 \mathrm{~Hz})$, $134.2(\mathrm{~d}, J=13.1 \mathrm{~Hz}), 131.9(\mathrm{~s}, 130.6,130.3(\mathrm{~d}, J=5.7 \mathrm{~Hz}), 129.7,124.2(\mathrm{~d}, J=16.8 \mathrm{~Hz}), 109.4$, 106.3, 105.8, 89.7, 89.5, 61.23 - 60.88 (m), 60.6 (d, $J=4.3 \mathrm{~Hz}), 56.1$, 33.2, 33.1 , 30.7 (d, $J=18.6$ Hz), $26.9(\mathrm{~d}, J=14.7 \mathrm{~Hz}), 18.5(\mathrm{t}, J=10.2 \mathrm{~Hz}) .{ }^{31} \mathrm{P}$ NMR $\left(162 \mathrm{MHz}, \mathrm{CDCl}_{3}\right) \delta-2.14$ (s). HRMS (ESI) calculated for $\left[\mathrm{M}+\mathrm{H}, \mathrm{C}_{40} \mathrm{H}_{50} \mathrm{O}_{9} \mathrm{P}\right]^{+}:$705.3187; found: 705.3180. Melting point: $160{ }^{\circ} \mathrm{C}$. 


\section{Preparation of substrates for Pd-catalyzed asymmetric intramolecular $\alpha$-arylation reactions and analytical data}

\subsection{Synthesis of 1a-p}

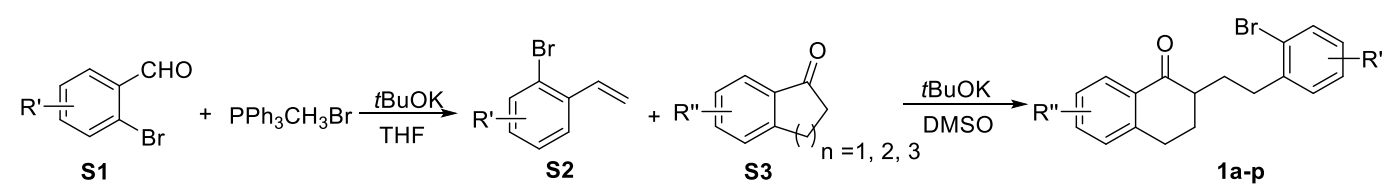

To a solution of the methyl triphenyphosphonium bromide ( $64.8 \mathrm{mmol}, 1.2$ equiv) in THF ( 80 $\mathrm{mL}$ ) at $0{ }^{\circ} \mathrm{C}$ under an $\mathrm{N}_{2}$ atmosphere, was added $t \mathrm{BuOK}$ (64.8 mmol, 1.2 equiv). The mixture was stirred at $0{ }^{\circ} \mathrm{C}$ for $1 \mathrm{~h}$ before the aldehyde $(54.0 \mathrm{mmol}, 1.0$ equiv) was added. The resulting mixture was allowed to warm to room temperature and stirred for hours until completion (monitored by TLC). The reaction mixture was diluted with petroleum ethers $(200 \mathrm{ml})$, filtered through a thin pad of silica gel, and washed with petroleum ether. The crude product was purified by silica gel column chromatography (petroleum ether) to afford pure product as yellow oil. ${ }^{1} \mathrm{H}$ NMR data of all compounds matched with those reports in the literature ${ }^{5}$.

The synthesis of 1a-p from $\mathbf{S 2}$ and $\mathbf{S 3}$ was according to procedures described in the literature ${ }^{6}$.

To a stirred solution of potassium tert-butoxide ( $6.5 \mathrm{mmol}, 0.4$ equiv.) in DMSO ( $0.5 \mathrm{M})$ were added $\mathbf{S 3}$ (49.2 mmol, 3.0 equiv.) followed by $\mathbf{S 2}$ (16.4 mmol, 1.0 equiv.). The reaction mixture was vigorously stirred for hours at room temperature. After complete consumption of $\mathbf{S 2}$ (monitored by TLC), the mixture was diluted with $\mathrm{H}_{2} \mathrm{O}(20 \mathrm{~mL})$ and extracted with ethyl acetate $(50 \mathrm{~mL} \times 3)$. The combined organic layer was washed with brine, dried over anhydrous $\mathrm{Na}_{2} \mathrm{SO}_{4}$ and filtrated. After the solvent was concentrated under reduced pressure, the crude product was purified by flash chromatography (silica gel, petroleum ether: ethyl acetate $=50: 1$ ) to afford the desired product.

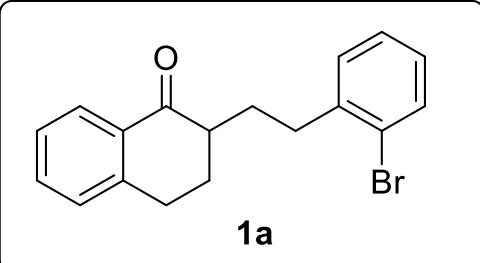

(2-Bromophenethyl)-3,4-dihydronaphthalen-1(2H)-one (1a): White solid. 60\% yield. (silica gel, petroleum ether: ethyl acetate $=50: 1) .{ }^{1} \mathrm{H}$ NMR $\left(500 \mathrm{MHz}, \mathrm{CDCl}_{3}\right) \delta 8.06-8.02(\mathrm{~m}, 1 \mathrm{H}), 7.52(\mathrm{dd}$, $J=8.0,1.0 \mathrm{~Hz}, 1 \mathrm{H}), 7.46(\mathrm{td}, J=7.5 \mathrm{~Hz}, J=1.3 \mathrm{~Hz}, 1 \mathrm{H}), 7.30(\mathrm{dd}, J=7.5 \mathrm{~Hz}, J=5.7 \mathrm{~Hz}, 2 \mathrm{H})$, $7.23(\mathrm{dd}, J=10.6 \mathrm{~Hz}, J=4.3 \mathrm{~Hz}, 2 \mathrm{H}), 7.05(\mathrm{td}, J=7.8 \mathrm{~Hz}, J=1.7 \mathrm{~Hz}, 1 \mathrm{H}), 3.03(\mathrm{dd}, J=9.7 \mathrm{~Hz}, J$ 
$=4.4 \mathrm{~Hz}, 2 \mathrm{H}), 2.92-2.86(\mathrm{~m}, 2 \mathrm{H}), 2.56(\mathrm{~m}, 1 \mathrm{H}), 2.37-2.31(\mathrm{~m}, 1 \mathrm{H}), 2.30-2.23(\mathrm{~m}, 1 \mathrm{H}), 2.05-$ $1.96(\mathrm{~m}, 1 \mathrm{H}), 1.86-1.78(\mathrm{~m}, 1 \mathrm{H}) .{ }^{13} \mathrm{C} \mathrm{NMR}\left(126 \mathrm{MHz}, \mathrm{CDCl}_{3}\right) \delta 200.0,143.9,141.4,133.2,132.8$, $132.5,130.5,128.7,127.6,127.5,127.4,126.6,124.4,47.0,33.5,29.9,28.5,28.5$. HRMS (ESI) calculated for [M+Na, $\left.\mathrm{C}_{18} \mathrm{H}_{17} \mathrm{BrO}\right]^{+}: 351.0355$; found: 351.0336 .

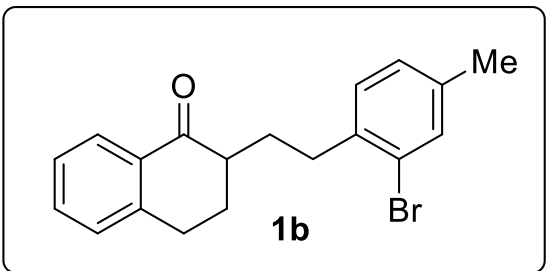

(2-Bromo-4-methylphenethyl)-3,4-dihydronaphthalen-1(2H)-one (1b): White solid. 58\% yield. (silica gel, petroleum ether: ethyl acetate $=50: 1) .{ }^{1} \mathrm{H} \mathrm{NMR}\left(500 \mathrm{MHz}, \mathrm{CDCl}_{3}\right) \delta 8.04(\mathrm{~d}, J=7.8$ Hz, 1H), $7.48-7.44$ (m, 1H), 7.35 (s, 1H), 7.30 (t, $J=7.5 \mathrm{~Hz}, 1 \mathrm{H}), 7.24$ (d, $J=7.6 \mathrm{~Hz}, 2 \mathrm{H}), 7.18$ $(\mathrm{d}, J=7.7 \mathrm{~Hz}, 1 \mathrm{H}), 7.04(\mathrm{~d}, J=7.8 \mathrm{~Hz}, 1 \mathrm{H}), 3.03(\mathrm{dd}, J=11.2 \mathrm{~Hz}, J=5.4 \mathrm{~Hz}, 2 \mathrm{H}), 2.88-2.79(\mathrm{~m}$, $2 \mathrm{H}), 2.58-2.51(\mathrm{~m}, 1 \mathrm{H}), 2.38-2.31(\mathrm{~m}, 1 \mathrm{H}), 2.29(\mathrm{~s}, 3 \mathrm{H}), 2.28-2.21(\mathrm{~m}, 1 \mathrm{H}), 1.99(\mathrm{~m}, 1 \mathrm{H}), 1.84$ $-1.75(\mathrm{~m}, 1 \mathrm{H}) .{ }^{13} \mathrm{C} \mathrm{NMR}\left(126 \mathrm{MHz}, \mathrm{CDCl}_{3}\right) \delta 200.0,143.9,138.2,137.6,133.2,133.1,132.5,130.2$, 128.7, 128.3, 127.4, 126.6, 124.1, 47.0, 33.0, 30.0, 28.5, 28.5, 20.6. HRMS (ESI) calculated for $\left[\mathrm{M}+\mathrm{Na}, \mathrm{C}_{19} \mathrm{H}_{19} \mathrm{BrNaO}_{2}\right]^{+}: 365.0511$; found: 365.0511 . Melting point: $72{ }^{\circ} \mathrm{C}$.

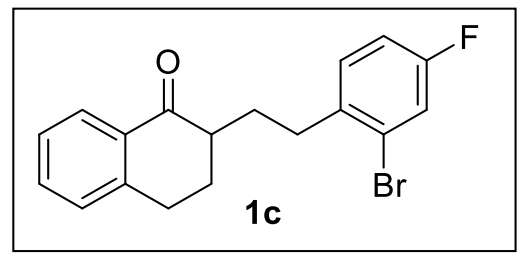

(2-Bromo-4-fluorophenethyl)-3,4-dihydronaphthalen-1(2H)-one(1c): White solid. 49\% yield. (silica gel, petroleum ether: ethyl acetate $=50: 1) .{ }^{1} \mathrm{H}$ NMR $\left(500 \mathrm{MHz}, \mathrm{CDCl}_{3}\right) \delta 8.04(\mathrm{~d}, J=7.8$ $\mathrm{Hz}, 1 \mathrm{H}), 7.47(\mathrm{t}, J=7.5 \mathrm{~Hz}, 1 \mathrm{H}), 7.32(\mathrm{~d}, J=7.6 \mathrm{~Hz}, 1 \mathrm{H}), 7.28(\mathrm{dd}, J=5.8 \mathrm{~Hz}, J=2.8 \mathrm{~Hz}, 2 \mathrm{H})$, $7.24(\mathrm{~d}, J=7.8 \mathrm{~Hz}, 1 \mathrm{H}), 6.96(\mathrm{td}, J=8.3 \mathrm{~Hz}, J=2.6 \mathrm{~Hz}, 1 \mathrm{H}), 3.03(\mathrm{dd}, J=9.1 \mathrm{~Hz}, J=4.4 \mathrm{~Hz}, 2 \mathrm{H})$, $2.87-2.82(\mathrm{~m}, 2 \mathrm{H}), 2.54(\mathrm{td}, J=11.3,5.7 \mathrm{~Hz}, 1 \mathrm{H}), 2.35-2.30(\mathrm{~m}, 1 \mathrm{H}), 2.27-2.19(\mathrm{~m}, 1 \mathrm{H}), 2.05$ $1.95(\mathrm{~m}, 1 \mathrm{H}), 1.79(\mathrm{~m}, 1 \mathrm{H}) .{ }^{13} \mathrm{C} \mathrm{NMR}\left(126 \mathrm{MHz}, \mathrm{CDCl}_{3}\right) \delta 199.87,160.8(J=239.4 \mathrm{~Hz}), 143.9$, 137.3, 133.2, 132.5, 131.0, 128.7, 127.4, 126.6, $124.1(J=12.6 \mathrm{~Hz}), 119.8(J=25.2 \mathrm{~Hz}), 114.6(J$ $=12.6 \mathrm{~Hz}), 47.0,32.7,30.0,28.6,28.5 .{ }^{19} \mathrm{~F}$ NMR $\left(376 \mathrm{MHz}, \mathrm{CDCl}_{3}\right) \delta-115.45$. HRMS (ESI) calculated for [M+H, $\left.\mathrm{C}_{18} \mathrm{H}_{17} \mathrm{BrFO}\right]^{+}$: 347.0441; found: 347.0436 . 


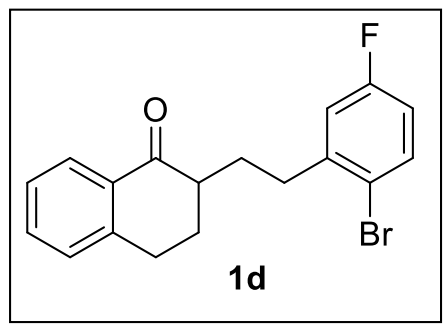

(2-Bromo-5-fluorophenethyl)-3,4-dihydronaphthalen-1(2H)-one(1d): White solid. 58\% yield. (silica gel, petroleum ether: ethyl acetate $=50: 1) .{ }^{1} \mathrm{H}$ NMR $\left(500 \mathrm{MHz}, \mathrm{CDCl}_{3}\right) \delta 8.04(\mathrm{~d}, J=7.8$ $\mathrm{Hz}, 1 \mathrm{H}), 7.47(\mathrm{dd}, J=8.7 \mathrm{~Hz}, J=5.5 \mathrm{~Hz}, 2 \mathrm{H}), 7.31(\mathrm{t}, J=7.5 \mathrm{~Hz}, 1 \mathrm{H}), 7.24(\mathrm{~d}, J=7.9 \mathrm{~Hz}, 1 \mathrm{H})$, $7.04(\mathrm{dd}, J=9.4,3.0 \mathrm{~Hz}, 1 \mathrm{H}), 6.79(\mathrm{td}, J=8.4 \mathrm{~Hz}, J=3.0 \mathrm{~Hz}, 1 \mathrm{H}), 3.07-3.01(\mathrm{~m}, 2 \mathrm{H}), 2.86(\mathrm{~m}$, $2 \mathrm{H}), 2.56(\mathrm{~m}, 1 \mathrm{H}), 2.37-2.22(\mathrm{~m}, 2 \mathrm{H}), 2.04-1.95(\mathrm{~m}, 1 \mathrm{H}), 1.81(\mathrm{~m}, 1 \mathrm{H}) .{ }^{13} \mathrm{C} \mathrm{NMR}(126 \mathrm{MHz}$, $\left.\mathrm{CDCl}_{3}\right) \delta 199.7,162.0(J=252.0 \mathrm{~Hz}), 143.8,143.6,137.8(\mathrm{~J}=12.6 \mathrm{~Hz}), 133.2,132.5,128.7,127.4$, $118.4,117.2(J=25.2 \mathrm{~Hz}), 114.8(J=12.6 \mathrm{~Hz}), 114.6(J=12.6 \mathrm{~Hz}), 47.0,33.6,29.7,28.6,28.5$. ${ }^{19} \mathrm{~F}$ NMR (376 MHz, $\left.\mathrm{CDCl}_{3}\right) \delta-115.16$ (s). HRMS (ESI) calculated for $\left[\mathrm{M}+\mathrm{H}, \mathrm{C}_{18} \mathrm{H}_{17} \mathrm{BrFO}\right]^{+}$: 347.0441; found:347.0444.

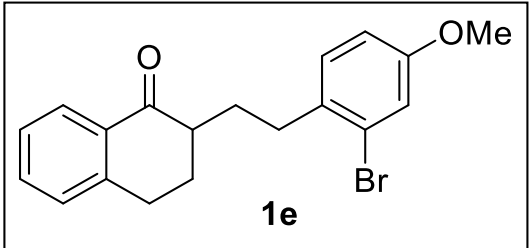

(2-Bromo-4-methoxyphenethyl)-3,4-dihydronaphthalen-1(2H)-one(1e): Brown solid. 50\% yield. (silica gel, petroleum ether: ethyl acetate $=50: 1) .{ }^{1} \mathrm{H}$ NMR $\left(500 \mathrm{MHz}, \mathrm{CDCl}_{3}\right) \delta 8.04(\mathrm{dd}, J$ $=7.8,1.0 \mathrm{~Hz}, 1 \mathrm{H}), 7.46(\mathrm{td}, J=7.5 \mathrm{~Hz}, J=1.3 \mathrm{~Hz}, 1 \mathrm{H}), 7.30(\mathrm{dd}, J=11.2 \mathrm{~Hz}, J=3.9 \mathrm{~Hz}, 1 \mathrm{H})$, $7.24(\mathrm{~d}, J=7.7 \mathrm{~Hz}, 1 \mathrm{H}), 7.20(\mathrm{~d}, J=8.5 \mathrm{~Hz}, 1 \mathrm{H}), 7.08(\mathrm{~d}, J=2.6 \mathrm{~Hz}, 1 \mathrm{H}), 6.80(\mathrm{dd}, J=8.5 \mathrm{~Hz}, J$ $=2.6 \mathrm{~Hz}, 1 \mathrm{H}), 3.77(\mathrm{~s}, 3 \mathrm{H}), 3.03(\mathrm{dd}, J=11.2 \mathrm{~Hz}, J=5.4 \mathrm{~Hz}, 2 \mathrm{H}), 2.85-2.78(\mathrm{~m}, 2 \mathrm{H}), 2.57-2.50$ $(\mathrm{m}, 1 \mathrm{H}), 2.33(\mathrm{~m}, 1 \mathrm{H}), 2.24(\mathrm{~m}, 1 \mathrm{H}), 2.03-1.94(\mathrm{~m}, 1 \mathrm{H}), 1.77(\mathrm{~m}, 1 \mathrm{H}) .{ }^{13} \mathrm{C} \mathrm{NMR}\left(126 \mathrm{MHz}, \mathrm{CDCl}_{3}\right)$ $\delta 200.0,158.3,143.9,133.3,133.1,132.5,130.7,128.7,127.4,126.6,124.4,117.9,113.7,55.5$, 46.9, 32.5, 30.1, 28.5, 28.5. HRMS (ESI) calculated for [M+Na, $\left.\mathrm{C}_{19} \mathrm{H}_{19} \mathrm{BrNaO}_{2}\right]^{+}: 381.0461$; found: 381.0465 .

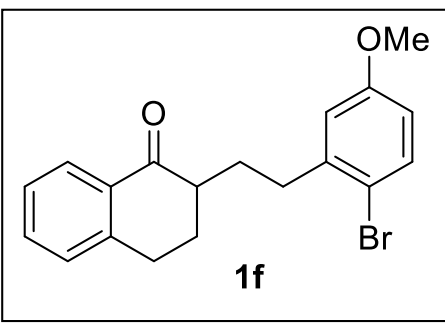


(2-Bromo-5-methoxyphenethyl)-3,4-dihydronaphthalen-1(2H)-one(1f): Brown solid. 53\% yield. (silica gel, petroleum ether: ethyl acetate $=50: 1) .{ }^{1} \mathrm{H} \mathrm{NMR}\left(500 \mathrm{MHz}, \mathrm{CDCl}_{3}\right) \delta 8.04(\mathrm{~d}, J=$ $7.7 \mathrm{~Hz}, 1 \mathrm{H}), 7.46(\mathrm{td}, J=7.5 \mathrm{~Hz}, J=1.1 \mathrm{~Hz}, 1 \mathrm{H}), 7.39$ (d, $J=8.7 \mathrm{~Hz}, 1 \mathrm{H}), 7.30$ (t, $J=7.5 \mathrm{~Hz}, 1 \mathrm{H})$, $7.24(\mathrm{~d}, J=7.7 \mathrm{~Hz}, 1 \mathrm{H}), 6.86(\mathrm{~d}, J=3.0 \mathrm{~Hz}, 1 \mathrm{H}), 6.63(\mathrm{dd}, J=8.7 \mathrm{~Hz}, J=3.0 \mathrm{~Hz}, 1 \mathrm{H}), 3.78(\mathrm{~s}$, $3 \mathrm{H}), 3.03(\mathrm{dd}, J=9.9,4.7 \mathrm{~Hz}, 2 \mathrm{H}), 2.87-2.80(\mathrm{~m}, 2 \mathrm{H}), 2.56(\mathrm{~m}, 1 \mathrm{H}), 2.34(\mathrm{~m}, 1 \mathrm{H}), 2.30-2.21$ $(\mathrm{m}, 1 \mathrm{H}), 2.04-1.96(\mathrm{~m}, 1 \mathrm{H}), 1.81(\mathrm{~m}, 1 \mathrm{H}) .{ }^{13} \mathrm{C} \mathrm{NMR}\left(126 \mathrm{MHz}, \mathrm{CDCl}_{3}\right) \delta 200.0,159.0,143.9$, 142.4, 133.2, 133.2, 132.5, 128.7, 127.4, 126.6, 115.9, 114.8, 113.5, 55.4, 47.0, 33.7, 29.9, 28.5, 28.5. HRMS (ESI) calculated for [M+H, $\left.\mathrm{C}_{19} \mathrm{H}_{20} \mathrm{BrO}_{2}\right]^{+}: 359.0641$; found: 359.0637 .

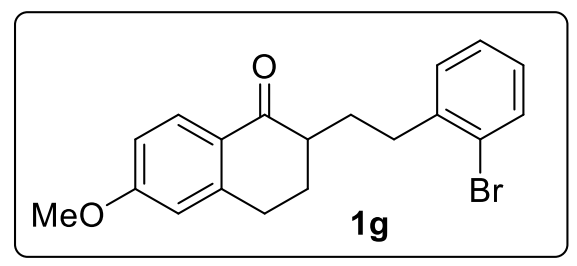

(2-Bromophenethyl)-6-methoxy-3,4-dihydronaphthalen-1(2H)-one(1g): Yellow oil. 60\% yield. (silica gel, petroleum ether: ethyl acetate $=50: 1) .{ }^{1} \mathrm{H}$ NMR $\left(500 \mathrm{MHz}, \mathrm{CDCl}_{3}\right) \delta 8.02(\mathrm{~d}, J=8.7$ $\mathrm{Hz}, 1 \mathrm{H}), 7.51(\mathrm{dd}, J=8.0 \mathrm{~Hz}, J=1.1 \mathrm{~Hz}, 1 \mathrm{H}), 7.31(\mathrm{dd}, J=7.6 \mathrm{~Hz}, J=1.7 \mathrm{~Hz}, 1 \mathrm{H}), 7.23(\operatorname{td}, J=$ $7.5 \mathrm{~Hz}, J=1.2 \mathrm{~Hz}, 1 \mathrm{H}), 7.06-7.02(\mathrm{~m}, 1 \mathrm{H}), 6.82(\mathrm{dd}, J=8.7 \mathrm{~Hz}, J=2.5 \mathrm{~Hz}, 1 \mathrm{H}), 6.69(\mathrm{~d}, J=2.5$ $\mathrm{Hz}, 1 \mathrm{H}), 3.85$ (s, 3H), $3.01-2.96(\mathrm{~m}, 2 \mathrm{H}), 2.87(\mathrm{~m}, 2 \mathrm{H}), 2.55-2.48(\mathrm{~m}, 1 \mathrm{H}), 2.35-2.20(\mathrm{~m}, 2 \mathrm{H})$, 2.02 - $1.95(\mathrm{~m}, 1 \mathrm{H}), 1.80(\mathrm{~m}, 1 \mathrm{H}) .{ }^{13} \mathrm{C} \mathrm{NMR}\left(126 \mathrm{MHz}, \mathrm{CDCl}_{3}\right) \delta 198.7,163.4,146.3,141.5,132.7$, 130.5, 129.9, 127.6, 127.5, 126.2, 124.4, 113.1,112.4, 55.4, 46.7, 33.6, 30.0, 28.8, 28.5. HRMS (ESI) calculated for $\left[\mathrm{M}+\mathrm{H}, \mathrm{C}_{19} \mathrm{H}_{20} \mathrm{BrO}_{2}\right]^{+}: 359.0641$; found: 359.0645 .

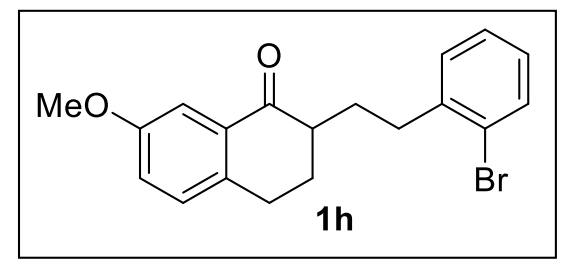

(2-Bromophenethyl)-7-methoxy-3,4-dihydronaphthalen-1(2H)-one(1h): White solid. 58\% yield. (silica gel, petroleum ether: ethyl acetate $=50: 1) .{ }^{1} 1 \mathrm{H} \mathrm{NMR}\left(500 \mathrm{MHz}, \mathrm{CDCl}_{3}\right) \delta 7.52(\mathrm{dd}, J=6.6$ $\mathrm{Hz}, J=2.0 \mathrm{~Hz}, 2 \mathrm{H}), 7.30(\mathrm{dd}, J=7.6 \mathrm{~Hz}, J=1.7 \mathrm{~Hz}, 1 \mathrm{H}), 7.25-7.22(\mathrm{~m}, 1 \mathrm{H}), 7.15(\mathrm{~d}, J=8.4 \mathrm{~Hz}$, 1H), 7.07 - $7.03(\mathrm{~m}, 2 \mathrm{H}), 3.84(\mathrm{~s}, 3 \mathrm{H}), 2.99-2.83(\mathrm{~m}, 4 \mathrm{H}), 2.54(\mathrm{~m}, 1 \mathrm{H}), 2.35-2.21(\mathrm{~m}, 2 \mathrm{H}), 2.03$ - $1.93(\mathrm{~m}, 1 \mathrm{H}), 1.85-1.77(\mathrm{~m}, 1 \mathrm{H}) .{ }^{13} \mathrm{C} \mathrm{NMR}\left(126 \mathrm{MHz}, \mathrm{CDCl}_{3}\right) \delta$ 199.9, 158.3, 141.4, 136.5, 133.3, $132.8,130.5,129.9,127.6,127.5,124.4,121.6,109.3,55.5,46.9,33.5,29.9,28.7,27.6$. HRMS (ESI) calculated for $\left[\mathrm{M}+\mathrm{H}, \mathrm{C}_{19} \mathrm{H}_{20} \mathrm{BrO}_{2}\right]^{+}: 359.0641$; found: 359.0637 . Melting point: $80{ }^{\circ} \mathrm{C}$. 


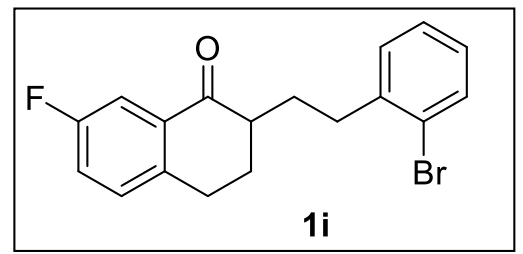

(2-Bromophenethyl)-7-fluoro-3,4-dihydronaphthalen-1(2H)-one(1i): Colorless oil. 58\%yield. (silica gel, petroleum ether: ethyl acetate $=50: 1) .{ }^{1} \mathrm{H}$ NMR $\left(500 \mathrm{MHz}, \mathrm{CDCl}_{3}\right) \delta 7.69(\mathrm{dd}, J=9.2$ $\mathrm{Hz}, J=2.7 \mathrm{~Hz}, 1 \mathrm{H}), 7.52(\mathrm{dd}, J=8.0 \mathrm{~Hz}, J=0.8 \mathrm{~Hz}, 1 \mathrm{H}), 7.30(\mathrm{dd}, J=7.6 \mathrm{~Hz}, J=1.6 \mathrm{~Hz}, 1 \mathrm{H})$, $7.25(\mathrm{~d}, J=0.9 \mathrm{~Hz}, 1 \mathrm{H}), 7.23-7.19(\mathrm{~m}, 1 \mathrm{H}), 7.17(\mathrm{dd}, J=8.2,2.8 \mathrm{~Hz}, 1 \mathrm{H}), 7.06(\mathrm{td}, J=7.8 \mathrm{~Hz}, J$ $=1.6 \mathrm{~Hz}, 1 \mathrm{H}), 3.04-2.94(\mathrm{~m}, 2 \mathrm{H}), 2.93-2.82(\mathrm{~m}, 2 \mathrm{H}), 2.56-2.50(\mathrm{~m}, 1 \mathrm{H}), 2.39-2.31(\mathrm{~m}, 1 \mathrm{H})$, $2.29-2.22(\mathrm{~m}, 1 \mathrm{H}), 1.98(\mathrm{~m}, 1 \mathrm{H}), 1.86-1.74(\mathrm{~m}, 1 \mathrm{H}) .{ }^{13} \mathrm{C} \mathrm{NMR}\left(126 \mathrm{MHz}, \mathrm{CDCl}_{3}\right) \delta 198.9,161.5$ $(J=247.0 \mathrm{~Hz}), 141.3,139.5,134.1,132.8,130.5,130.4,127.7,127.6,124.0,120.5(J=22.7 \mathrm{~Hz})$, $113.3(J=22.7 \mathrm{~Hz}), 46.7,33.4,29.7,28.5,27.7 .{ }^{19} \mathrm{~F} \mathrm{NMR}\left(376 \mathrm{MHz}, \mathrm{CDCl}_{3}\right) \delta-115.34$. HRMS (ESI) calculated for [M+Na, $\left.\mathrm{C}_{18} \mathrm{H}_{16} \mathrm{BrFNaO}\right]^{+}: 369.0261$; found: 369.0259 .

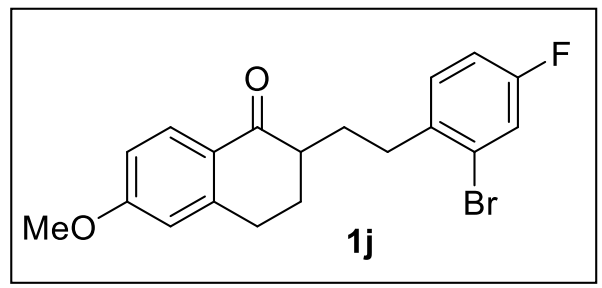

(2-Bromo-4-fluorophenethyl)-6-methoxy-3,4-dihydronaphthalen-1(2H)-one(1j): Yellow oil. $50 \%$ yield. (silica gel, petroleum ether: ethyl acetate $=50: 1) .{ }^{1} \mathrm{H} \mathrm{NMR}\left(500 \mathrm{MHz}, \mathrm{CDCl}_{3}\right) \delta 8.01$ $(\mathrm{d}, J=8.7 \mathrm{~Hz}, 1 \mathrm{H}), 7.28(\mathrm{dd}, J=5.4 \mathrm{~Hz}, J=3.1 \mathrm{~Hz}, 1 \mathrm{H}), 7.26(\mathrm{~s}, 1 \mathrm{H}), 6.98-6.94(\mathrm{~m}, 1 \mathrm{H}), 6.83$ $(\mathrm{dd}, J=8.7 \mathrm{~Hz}, J=2.5 \mathrm{~Hz}, 1 \mathrm{H}), 6.69(\mathrm{~d}, J=2.4 \mathrm{~Hz}, 1 \mathrm{H}), 3.85(\mathrm{~s}, 3 \mathrm{H}), 2.99(\mathrm{~m}, 2 \mathrm{H}), 2.84(\mathrm{~m}, 2 \mathrm{H})$, $2.49(\mathrm{~m}, 1 \mathrm{H}), 2.33-2.17(\mathrm{~m}, 2 \mathrm{H}), 2.01-1.93(\mathrm{~m}, 1 \mathrm{H}), 1.77(\mathrm{~m}, 1 \mathrm{H}) .{ }^{13} \mathrm{C} \mathrm{NMR}\left(126 \mathrm{MHz}, \mathrm{CDCl}_{3}\right)$ $\delta 198.6,163.4,160.8(J=239.4 \mathrm{~Hz}), 146.3,137.4(J=12.6 \mathrm{~Hz}), 131.0,129.9,126.1,124.1(J=$ $12.6 \mathrm{~Hz}), 119.8(J=12.6 \mathrm{~Hz}), 114.5(J=25.2 \mathrm{~Hz}), 113.1,112.4,55.39,46.63,32.76,30.12,28.85$, 28.58. ${ }^{19} \mathrm{~F}$ NMR $\left(376 \mathrm{MHz}, \mathrm{CDCl}_{3}\right) \delta$-115.30. HRMS (ESI) calculated for $\left[\mathrm{M}+\mathrm{H}, \mathrm{C}_{19} \mathrm{H}_{19} \mathrm{BrFO}_{2}\right]^{+}$: 377.0547; found: 377.0540 . Melting point: $90^{\circ} \mathrm{C}$.

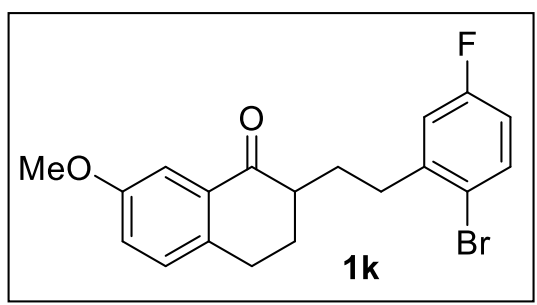


(2-Bromo-5-fluorophenethyl)-7-methoxy-3,4-dihydronaphthalen-1(2H)-one(1k): White solid. $70 \%$ yield. (silica gel, petroleum ether: ethyl acetate $=50: 1) .{ }^{1} \mathrm{H} \mathrm{NMR}\left(500 \mathrm{MHz}, \mathrm{CDCl}_{3}\right) \delta 7.52$ $(\mathrm{d}, J=2.8 \mathrm{~Hz}, 1 \mathrm{H}), 7.47(\mathrm{dd}, J=8.7 \mathrm{~Hz}, J=5.4 \mathrm{~Hz}, 1 \mathrm{H}), 7.15(\mathrm{~d}, J=8.4 \mathrm{~Hz}, 1 \mathrm{H}), 7.05(\mathrm{~m}, 2 \mathrm{H})$, $6.79(\mathrm{td}, J=8.3 \mathrm{~Hz}, J=3.0 \mathrm{~Hz}, 1 \mathrm{H}), 3.84(\mathrm{~s}, 3 \mathrm{H}), 2.97(\mathrm{~m}, 2 \mathrm{H}), 2.85(\mathrm{~m}, 2 \mathrm{H}), 2.53(\mathrm{~m}, 1 \mathrm{H}), 2.34$ $2.19(\mathrm{~m}, 2 \mathrm{H}), 2.02-1.92(\mathrm{~m}, 1 \mathrm{H}), 1.85-1.76(\mathrm{~m}, 1 \mathrm{H}) .{ }^{13} \mathrm{C} \mathrm{NMR}\left(126 \mathrm{MHz}, \mathrm{CDCl}_{3}\right) \delta 199.7,162.0$ $(J=252 \mathrm{~Hz}), 158.3,143.6,136.4,133.8(J=12.6 \mathrm{~Hz}), 133.2,129.9,121.6,118.4,117.2(J=25.2$ $\mathrm{Hz}), 114.8(J=12.6 \mathrm{~Hz}), 109.3,55.5,46.8,33.7,29.7,28.8,27.7 .{ }^{19} \mathrm{~F}$ NMR $\left(376 \mathrm{MHz}, \mathrm{CDCl}_{3}\right) \delta-$ 115.36. HRMS (ESI) calculated for $\left[\mathrm{M}+\mathrm{H}, \mathrm{C}_{19} \mathrm{H}_{18} \mathrm{BrFNaO}_{2}\right]^{+}$: 399.0366; found: 399.0362 . Melting point: $70^{\circ} \mathrm{C}$.

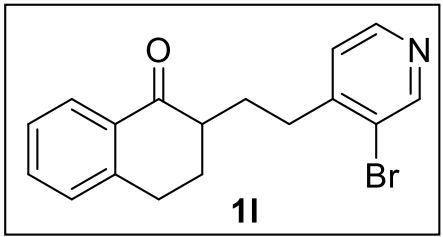

(2-(3-Bromopyridin-4-yl)ethyl)-3,4-dihydronaphthalen-1(2H)-one(11): Brown solid. 30\% yield. ( silica gel, petroleum ether: ethyl acetate $=50: 1) .{ }^{1} \mathrm{H}$ NMR $\left(500 \mathrm{MHz}, \mathrm{CDCl}_{3}\right) \delta 8.62(\mathrm{~s}, 1 \mathrm{H}), 8.38$ $(\mathrm{d}, J=4.9 \mathrm{~Hz}, 1 \mathrm{H}), 8.01(\mathrm{dd}, J=7.9 \mathrm{~Hz}, J=1.1 \mathrm{~Hz}, 1 \mathrm{H}), 7.47-7.41(\mathrm{~m}, 1 \mathrm{H}), 7.31-7.26(\mathrm{~m}, 1 \mathrm{H})$, $7.22(\mathrm{t}, J=5.8 \mathrm{~Hz}, 2 \mathrm{H}), 3.01(\mathrm{~m}, 2 \mathrm{H}), 2.87-2.82(\mathrm{~m}, 2 \mathrm{H}), 2.52(\mathrm{~m}, 1 \mathrm{H}), 2.25(\mathrm{~m}, 2 \mathrm{H}), 2.01-1.92$ (m, 1H), $1.85-1.76(\mathrm{~m}, 1 \mathrm{H}) .{ }^{13} \mathrm{C}$ NMR $\left(126 \mathrm{MHz}, \mathrm{cdcl}_{3}\right) \delta 199.5,151.8,150.3,148.3,143.8,133.3$, 132.4, 128.7, 127.4, 126.7, 125.2, 123.1, 46.9, 32.7, 29.0, 28.7, 28.6. HRMS (ESI) calculated for $\left[\mathrm{M}+\mathrm{H}, \mathrm{C}_{17} \mathrm{H}_{17} \mathrm{BrNO}\right]^{+}: 330.0488$; found: 330.0490 . Melting point: $85^{\circ} \mathrm{C}$.

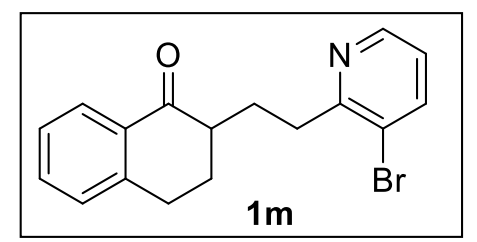

(2-(3-Bromopyridin-2-yl)ethyl)-3,4-dihydronaphthalen-1(2H)-one(1m): Yellow solid. $23 \%$ yield. (silica gel, petroleum ether: ethyl acetate $=50: 1) .{ }^{1} \mathrm{H} \mathrm{NMR}\left(500 \mathrm{MHz}, \mathrm{CDCl}_{3}\right) \delta$ $8.46(\mathrm{dd}, J=4.7 \mathrm{~Hz}, J=1.2 \mathrm{~Hz}, 1 \mathrm{H}), 8.03(\mathrm{~d}, J=7.8 \mathrm{~Hz}, 1 \mathrm{H}), 7.82$ (d, $J=8.0 \mathrm{~Hz}, 1 \mathrm{H}), 7.45$ (td, $J$ $=7.5 \mathrm{~Hz}, J=1.2 \mathrm{~Hz}, 1 \mathrm{H}), 7.29(\mathrm{t}, J=7.6 \mathrm{~Hz}, 1 \mathrm{H}), 7.23(\mathrm{~d}, J=7.6 \mathrm{~Hz}, 1 \mathrm{H}), 7.01(\mathrm{dd}, J=8.0 \mathrm{~Hz}, J$ $=4.7 \mathrm{~Hz}, 1 \mathrm{H}), 3.15-3.09(\mathrm{~m}, 2 \mathrm{H}), 3.03(\mathrm{~m}, 2 \mathrm{H}), 2.61(\mathrm{~m}, 1 \mathrm{H}), 2.47-2.33(\mathrm{~m}, 2 \mathrm{H}), 2.05-1.98(\mathrm{~m}$, 1H), $1.96-1.89(\mathrm{~m}, 1 \mathrm{H}) .{ }^{13} \mathrm{C} \mathrm{NMR}\left(126 \mathrm{MHz}, \mathrm{CDCl}_{3}\right) \delta 199.8,160.1,147.6,143.9,140.3,133.1$, 132.5, 128.6, 127.5, 126.5, 122.5, 121.4, 47.1, 34.9, 28.5, 28.5, 28.2. HRMS (ESI) calculated for [M+H, $\left.\mathrm{C}_{17} \mathrm{H}_{17} \mathrm{BrNO}\right]^{+}:$330.0488; found: 330.0491. Melting point: $85^{\circ} \mathrm{C}$. 


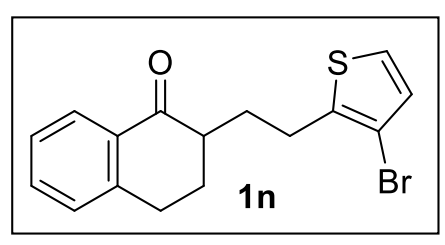

(2-(3-Bromothiophen-2-yl)ethyl)-3,4-dihydronaphthalen-1(2H)-one(1n):White solid.41\% yield. (silica gel, petroleum ether: ethyl acetate $=50: 1) .{ }^{1} \mathrm{H} \mathrm{NMR}\left(500 \mathrm{MHz}, \mathrm{CDCl}_{3}\right) \delta 8.04(\mathrm{~d}, J=7.8$ $\mathrm{Hz}, 1 \mathrm{H}), 7.46(\mathrm{t}, J=7.5 \mathrm{~Hz}, 1 \mathrm{H}), 7.30(\mathrm{t}, J=7.5 \mathrm{~Hz}, 1 \mathrm{H}), 7.23(\mathrm{~d}, J=7.6 \mathrm{~Hz}, 1 \mathrm{H}), 7.12(\mathrm{~d}, J=5.3$ $\mathrm{Hz}, 1 \mathrm{H}), 6.90(\mathrm{~d}, J=5.3 \mathrm{~Hz}, 1 \mathrm{H}), 3.02(\mathrm{dd}, J=7.3 \mathrm{~Hz}, J=4.7 \mathrm{~Hz}, 2 \mathrm{H}), 2.99-2.94(\mathrm{~m}, 2 \mathrm{H}), 2.54$ (m, 1H), $2.41-2.28(\mathrm{~m}, 2 \mathrm{H}), 1.95(\mathrm{~m}, 1 \mathrm{H}), 1.82(\mathrm{~m}, 1 \mathrm{H}) .{ }^{13} \mathrm{C} \mathrm{NMR}\left(126 \mathrm{MHz}, \mathrm{CDCl}_{3}\right) \delta$ 199.6, 143.8, 139.1, 133.2, 132.5, 129.9, 128.7, 127.4, 126.6, 123.3, 108.8, 46.6, 30.6, 28.7, 28.6, 26.7. HRMS (ESI) calculated for [M+Na, $\left.\mathrm{C}_{16} \mathrm{H}_{15} \mathrm{BrNaOS}\right]^{+}$: 356.9919; found: 356.9920. Melting point: $64{ }^{\circ} \mathrm{C}$.

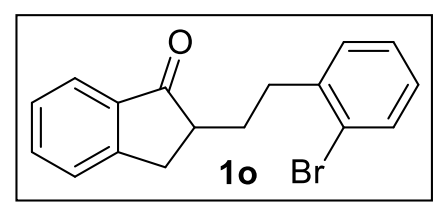

(2-Bromophenethyl)-2,3-dihydro-1H-inden-1-one(10): Yellow oil. 37\%yield. (silica gel, petroleum ether: ethyl acetate $=50: 1) .{ }^{1} \mathrm{H}$ NMR $\left(500 \mathrm{MHz}, \mathrm{CDCl}_{3}\right) \delta 7.76(\mathrm{~d}, J=7.6 \mathrm{~Hz}, 1 \mathrm{H}), 7.59$ $(\mathrm{td}, J=7.5 \mathrm{~Hz}, J=1.2 \mathrm{~Hz}, 1 \mathrm{H}), 7.53(\mathrm{dd}, J=8.0 \mathrm{~Hz}, J=1.2 \mathrm{~Hz}, 1 \mathrm{H}), 7.49-7.47(\mathrm{~m}, 1 \mathrm{H}), 7.39$ $7.36(\mathrm{~m}, 1 \mathrm{H}), 7.29(\mathrm{dd}, J=7.6 \mathrm{~Hz}, J=1.8 \mathrm{~Hz}, 1 \mathrm{H}), 7.24(\mathrm{td}, J=7.4 \mathrm{~Hz}, J=1.2 \mathrm{~Hz}, 1 \mathrm{H}), 7.08$ $7.04(\mathrm{~m}, 1 \mathrm{H}), 3.40$ (dd, $J=17.2 \mathrm{~Hz}, J=7.9 \mathrm{~Hz}, 1 \mathrm{H}), 2.97-2.85$ (m, 4H), $2.72(\mathrm{~m}, 1 \mathrm{H}), 2.30-2.22$ (m, 1H), $1.79(\mathrm{~m}, 1 \mathrm{H}) .{ }^{13} \mathrm{C} \mathrm{NMR}\left(126 \mathrm{MHz}, \mathrm{CDCl}_{3}\right) \delta 208.3,153.6,140.9,136.7,134.7,132.8$, 130.5, 127.8, 127.5, 127.4, 126.5, 124.4, 123.9, 46.9, 33.9, 33.0, 31.7. HRMS (ESI) calculated for $\left[\mathrm{M}+\mathrm{Na}, \mathrm{C}_{17} \mathrm{H}_{15} \mathrm{BrNaO}\right]^{+}: 337.0198$; found:337.0197.

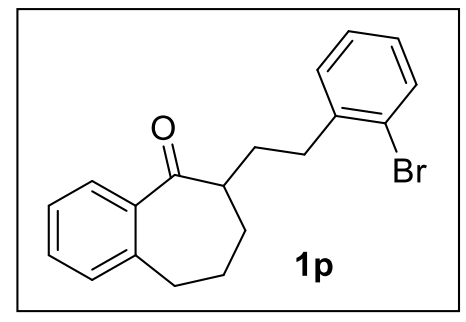

(2-Bromophenethyl)-6,7,8,9-tetrahydro-5H-benzo[7] annulen-5-one(1p): Yellow oil. 65\% yield. Colorless oil. (silica gel, petroleum ether: ethyl acetate $=50: 1) .{ }^{1} \mathrm{H} \mathrm{NMR}\left(500 \mathrm{MHz}, \mathrm{CDCl}_{3}\right) \delta 7.67$ $(\mathrm{dd}, J=7.7 \mathrm{~Hz}, J=1.3 \mathrm{~Hz}, 1 \mathrm{H}), 7.51-7.48(\mathrm{~m}, 1 \mathrm{H}), 7.38(\mathrm{td}, J=7.5 \mathrm{~Hz}, J=1.4 \mathrm{~Hz}, 1 \mathrm{H}), 7.29$ (t, $J=7.4 \mathrm{~Hz}, 1 \mathrm{H}), 7.24-7.18(\mathrm{~m}, 3 \mathrm{H}), 7.03(\mathrm{ddd}, J=8.0 \mathrm{~Hz}, J=6.8 \mathrm{~Hz}, J=2.3 \mathrm{~Hz}, 1 \mathrm{H}), 3.05-2.87$ $(\mathrm{m}, 4 \mathrm{H}), 2.76-2.71(\mathrm{~m}, 2 \mathrm{H}), 2.24(\mathrm{~m}, 1 \mathrm{H}), 2.14-2.00(\mathrm{~m}, 2 \mathrm{H}), 1.86-1.76(\mathrm{~m}, 1 \mathrm{H}), 1.75-1.63(\mathrm{~m}$, 
2H). ${ }^{13} \mathrm{C}$ NMR (126 MHz, $\left.\mathrm{CDCl}_{3}\right) \delta 207.0,141.9,141.4,140.1,132.7,131.2,130.4,129.8,128.3$, 127.5, 127.4, 126.3, 124.4, 49.4, 33.8, 33.7, 31.4, 30.4, 25.4. HRMS (ESI) calculated for $[\mathrm{M}+\mathrm{H}$, $\mathrm{C}_{19} \mathrm{H}_{20} \mathrm{BrO}^{+}:$343.0692; found: 343.0690 .

\subsection{Synthesis of 1q-z}

1q-z were prepared as follows.
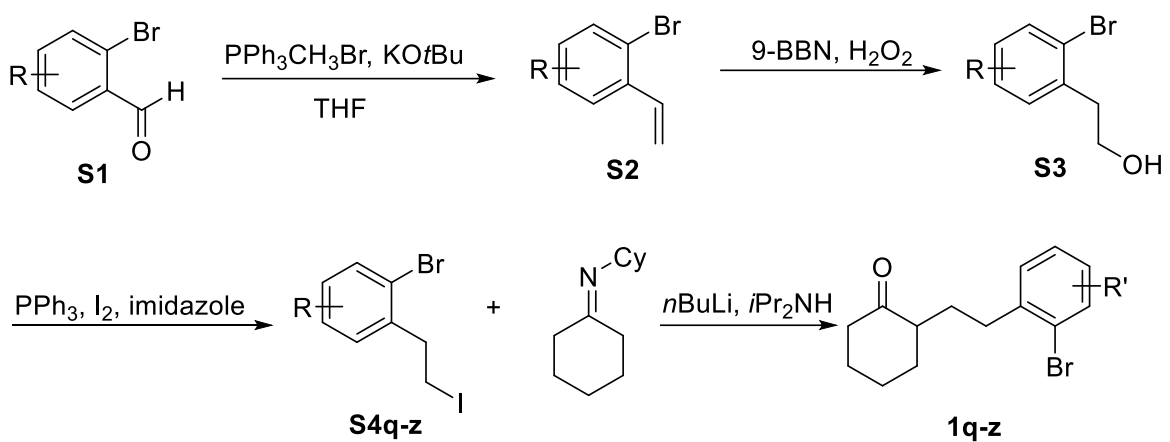

To a solution of 9-BBN (35.2 mmol,1.5 equiv.) in dry THF was added dropwise $\mathbf{S 2}$ (23.5 mmol, 1.0 equiv) under $\mathrm{N}_{2}$ and the mixture was stirred at room temperature for $12 \mathrm{~h}$. Then aqueous $\mathrm{NaOH}$ solution $(6 \mathrm{M})$ was added to the reaction mixture slowly under the ice-bath temperature. After stirring for 1 hour, $\mathrm{H}_{2} \mathrm{O}_{2}(30 \%, 5 \mathrm{~mL})$ was added at the same temperature. To the solvent was added saturated aqueous $\mathrm{Na}_{2} \mathrm{~S}_{2} \mathrm{O}_{3}(20 \mathrm{~mL})$, and then extracted with $\mathrm{EA}(50 \mathrm{~mL} \times 3)$. The combined organic layer was washed with brine, dried over anhydrous $\mathrm{Na}_{2} \mathrm{SO}_{4}$ and filtrated. After the solvent was concentrated under reduced pressure, the crude product was purified by silica gel column chromatography (petroleum ether: ethyl acetate $=20: 1-4: 1$ ) to afford the desired product as pale yellow oil.

To a solution of $\mathrm{PPh}_{3}$ (28.3 mmol,1.5 equiv) in DCM was added $\mathrm{I}_{2}$ under $\mathrm{N}_{2}$ and the mixture was stirred at room temperature for $10 \mathrm{~min}$. Then imidazole ( $47.0 \mathrm{mmol}, 2.5$ equiv) was added to the reaction mixture and the mixture was stirred at room temperature for another $10 \mathrm{~min}$. Then add $\mathbf{S 3}$ (18.8 mmol, 1 equiv) to the reaction mixture. After completion (monitored by TLC), the reaction mixture was quenched with saturated aqueous $\mathrm{Na}_{2} \mathrm{~S}_{2} \mathrm{O}_{3}$ solution and diluted with $\mathrm{H}_{2} \mathrm{O}(20 \mathrm{~mL})$ and extracted with DCM (50 mL x 3). The combined organic layer was washed with brine, dried over anhydrous $\mathrm{Na}_{2} \mathrm{SO}_{4}$ and filtrated. After the solvent was concentrated under reduced pressure, the 
crude product was purified by flash chromatography (silica gel, petroleum ether) to afford the desired product $\mathbf{S 4 q - z .}$. S4y was matched with reported data?

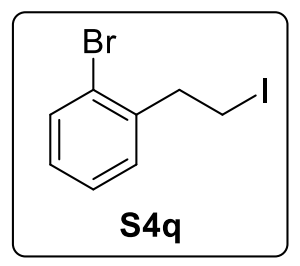

1-Bromo-2-(2-iodoethyl)benzene (S4q): Brown oil. 80\% yield. (silica gel, eluent, petroleum ether). ${ }^{1} \mathrm{H}$ NMR $\left(500 \mathrm{MHz}, \mathrm{CDCl}_{3}\right) \delta 7.55(\mathrm{dd}, J=8.0, J=0.9 \mathrm{~Hz}, 1 \mathrm{H}), 7.30-7.23(\mathrm{~m}, 2 \mathrm{H}), 7.17-7.11$ (m, 1H), $3.41-3.34(\mathrm{~m}, 2 \mathrm{H}), 3.33-3.28(\mathrm{~m}, 2 \mathrm{H}) .{ }^{13} \mathrm{C} \mathrm{NMR}\left(126 \mathrm{MHz}, \mathrm{CDCl}_{3}\right) \delta$ 139.8, 133.0, 130.7, 128.6, 127.6, 124.0, 40.5, 3.2. HRMS (EI): $\mathrm{m} / \mathrm{z}$ calculated for $\left[\mathrm{M}, \mathrm{C}_{8} \mathrm{H}_{8} \mathrm{BrI}\right]^{+}:$: 309.8854; found: 309.8846 .

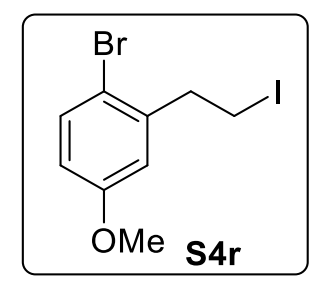

1-Bromo-2-(2-iodoethyl)-4-methoxybenzene (S4r): Yellow oil. 80\% yield. (silica gel, eluent, petroleum ether). ${ }^{1} \mathrm{H}$ NMR $\left(500 \mathrm{MHz}, \mathrm{CDCl}_{3}\right) \delta 7.42(\mathrm{~d}, J=8.8 \mathrm{~Hz}, 1 \mathrm{H}), 6.79(\mathrm{~d}, J=3.0 \mathrm{~Hz}, 1 \mathrm{H})$, $6.70(\mathrm{dd}, J=8.8, J=3.0 \mathrm{~Hz}, 1 \mathrm{H}), 3.79(\mathrm{~s}, 3 \mathrm{H}), 3.36(\mathrm{t}, J=7.7 \mathrm{~Hz}, 2 \mathrm{H}), 3.25(\mathrm{t}, J=7.8 \mathrm{~Hz}, 2 \mathrm{H}) .{ }^{13} \mathrm{C}$ NMR $\left(126 \mathrm{MHz}, \mathrm{CDCl}_{3}\right) \delta 158.9,140.7,133.5,116.3,114.3,114.1,55.4,40.7,3.0$. HRMS (EI): $\mathrm{m} / \mathrm{z}$ calculated for $\left[\mathrm{M}, \mathrm{C}_{9} \mathrm{H}_{10} \mathrm{BrIO}\right]^{+}: 339.8960$; found: 339.8956 .

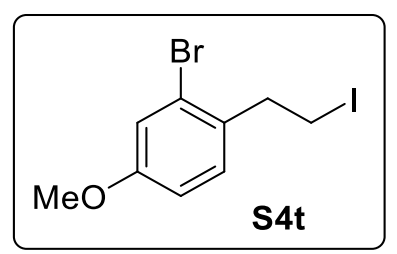

2-Bromo-1-(2-iodoethyl)-4-methoxybenzene (S4t): Yellow oil. 85\% yield. (silica gel, eluent, petroleum ether) ${ }^{1} \mathrm{H}$ NMR $\left(500 \mathrm{MHz}, \mathrm{CDCl}_{3}\right) \delta 7.07(\mathrm{~d}, J=8.5 \mathrm{~Hz}, 1 \mathrm{H}), 7.02(\mathrm{~d}, J=2.5 \mathrm{~Hz}, 1 \mathrm{H})$, $6.74(\mathrm{dd}, J=8.4, J=2.5 \mathrm{~Hz}, 1 \mathrm{H}), 3.71(\mathrm{~s}, 3 \mathrm{H}), 3.26(\mathrm{t}, J=7.7 \mathrm{~Hz}, 2 \mathrm{H}), 3.15(\mathrm{t}, J=7.7 \mathrm{~Hz}, 2 \mathrm{H}){ }^{13} \mathrm{C}$ $\operatorname{NMR}\left(126 \mathrm{MHz}, \mathrm{CDCl}_{3}\right) \delta 159.0,131.9,131.0,124.2,118.2,113.6,55.5,39.7,4.1 . H R M S(E I)$ : $\mathrm{m} / \mathrm{z}$ calculated for $\left[\mathrm{M}, \mathrm{C}_{9} \mathrm{H}_{10} \mathrm{BrIO}\right]^{+}: 339.8960$; found: 339.8958 . 


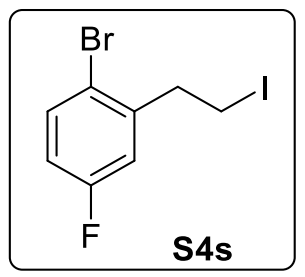

1-Bromo-4-fluoro-2-(2-iodoethyl)benzene (S4s): Yellow oil. $42 \%$ yield. (silica gel, eluent, petroleum ether). ${ }^{1} \mathrm{H} \mathrm{NMR}\left(500 \mathrm{MHz}, \mathrm{CDCl}_{3}\right) \delta 7.50(\mathrm{dd}, J=9.0,1 \mathrm{H}), 6.99(\mathrm{dd}, J=9.0, J=3.0 \mathrm{~Hz}$, $1 \mathrm{H}), 6.93-6.82(\mathrm{~m}, 1 \mathrm{H}), 3.39-3.34(\mathrm{~m}, 2 \mathrm{H}), 3.30-3.24(\mathrm{~m}, 2 \mathrm{H}) .{ }^{13} \mathrm{C} \mathrm{NMR}\left(126 \mathrm{MHz}, \mathrm{CDCl}_{3}\right) \delta$ $161.9(J=239.4 \mathrm{~Hz}), 141.8,134.2,118.1,117.6,115.8,40.4,2.2 .{ }^{19} \mathrm{~F} \mathrm{NMR}\left(376 \mathrm{MHz}, \mathrm{CDCl}_{3}\right) \delta-$ 115.52. HRMS (EI): $\mathrm{m} / \mathrm{z}$ calculated for $\left[\mathrm{M}, \mathrm{C}_{8} \mathrm{H}_{7} \mathrm{FBrI}\right]^{+}: 327.8760$; found: 327.8758 .

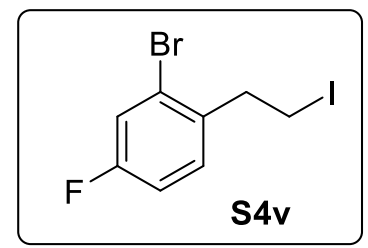

2-Bromo-4-fluoro-1-(2-iodoethyl)benzene (S4v):Yellow oil. 70\% yield. (silica gel, eluent, petroleum ether) ${ }^{1} \mathrm{H} \mathrm{NMR}\left(500 \mathrm{MHz}, \mathrm{CDCl}_{3}\right) \delta 7.30(\mathrm{dd}, J=8.2, J=2.6 \mathrm{~Hz}, 1 \mathrm{H}), 7.22(\mathrm{dd}, J=$ $8.5, J=6.0 \mathrm{~Hz}, 1 \mathrm{H}), 7.04-6.97(\mathrm{~m}, 1 \mathrm{H}), 3.41-3.30(\mathrm{~m}, 2 \mathrm{H}), 3.32-3.21(\mathrm{~m}, 2 \mathrm{H}) .{ }^{13} \mathrm{C}$ NMR $(151$ $\left.\mathrm{MHz}, \mathrm{CDCl}_{3}\right) \delta 160.3(J=209.2 \mathrm{~Hz}), 134.7,130.4,122.9,119.2,113.7,38.5,2.2 .{ }^{19} \mathrm{~F}$ NMR $(376$ $\left.\mathrm{MHz}, \mathrm{CDCl}_{3}\right) \delta-115.74$. HRMS (EI): $\mathrm{m} / \mathrm{z}$ calculated for $\left[\mathrm{M}, \mathrm{C}_{8} \mathrm{H}_{7} \mathrm{FBrI}\right]^{+}:$327.8760; found: 327.8764 .

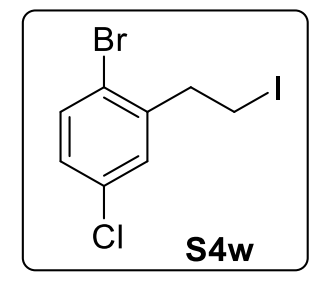

1-Bromo-4-chloro-2-(2-iodoethyl)benzene (S4w): Yellow oil. 45\% yield. (silica gel, eluent, petroleum ether) ${ }^{1} \mathrm{H} \mathrm{NMR}\left(500 \mathrm{MHz}, \mathrm{CDCl}_{3}\right) \delta 7.46(\mathrm{~d}, J=8.5 \mathrm{~Hz}, 1 \mathrm{H}), 7.23(\mathrm{~d}, J=2.5 \mathrm{~Hz}, 1 \mathrm{H})$, $7.12(\mathrm{dd}, J=8.5, J=2.5 \mathrm{~Hz}, 1 \mathrm{H}), 3.34(\mathrm{dd}, J=11.6 \mathrm{~Hz}, J=4.5 \mathrm{~Hz}, 2 \mathrm{H}), 3.25(\mathrm{dd}, J=11.5 \mathrm{~Hz}, J=$ $4.1 \mathrm{~Hz}, 2 \mathrm{H}) .{ }^{13} \mathrm{C} \mathrm{NMR}\left(126 \mathrm{MHz}, \mathrm{CDCl}_{3}\right) \delta 141.4,134.0,133.4,130.5,128.7,121.9,40.2,2.2$. HRMS (EI): $\mathrm{m} / \mathrm{z}$ calculated for $\left[\mathrm{M}, \mathrm{C}_{8} \mathrm{H}_{7} \mathrm{ClBrI}\right]^{+}: 343.8464$; found: 343.8466 . 


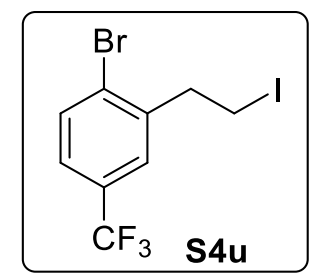

1-Bromo-2-(2-iodoethyl)-4-(trifluoromethyl)benzene (S4u): Yellow oil. 52\% yield. (silica gel, eluent, petroleum ether). ${ }^{1} \mathrm{H}$ NMR $\left(500 \mathrm{MHz}, \mathrm{CDCl}_{3}\right) \delta 7.68(\mathrm{~d}, J=8.3 \mathrm{~Hz}, 1 \mathrm{H}), 7.50(\mathrm{~d}, J=1.8$ $\mathrm{Hz}, 1 \mathrm{H}), 7.40(\mathrm{dd}, J=8.3 \mathrm{~Hz}, J=2.0 \mathrm{~Hz}, 1 \mathrm{H}), 3.41-3.37(\mathrm{~m}, 2 \mathrm{H}), 3.37-3.33(\mathrm{~m}, 2 \mathrm{H}) .{ }^{13} \mathrm{C}$ NMR $\left(151 \mathrm{MHz}, \mathrm{CDCl}_{3}\right) \delta 140.9,133.8,130.2(J=33 \mathrm{~Hz}), 128.1,127.5,125.4,123.8(J=270 \mathrm{~Hz}), 40.4$, 2.1. ${ }^{19} \mathrm{~F}$ NMR (376 MHz, $\left.\mathrm{CDCl}_{3}\right) \delta-62.24$. HRMS (EI): $\mathrm{m} / \mathrm{z}$ calculated for $\left[\mathrm{M}, \mathrm{C}_{9} \mathrm{H}_{7} \mathrm{~F}_{3} \mathrm{BrI}\right]^{+}$: 377.8728; found: 377.8752 .

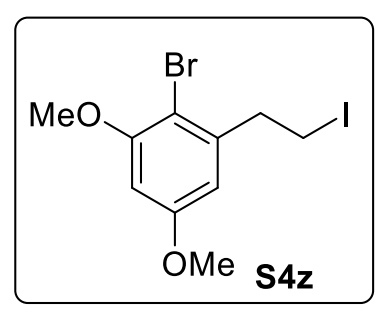

1-Bromo-2-(2-iodoethyl)-4,5-dimethoxybenzene (S4z): White solid. 62\% yield. (silica gel, eluent, petroleum ether/ethyl acetate $=20: 1) .{ }^{1} \mathrm{H}$ NMR $\left(500 \mathrm{MHz}, \mathrm{CDCl}_{3}\right) \delta 6.42(\mathrm{~m}, 2 \mathrm{H}), 3.86(\mathrm{~s}$, $3 \mathrm{H}), 3.81(\mathrm{~s}, 3 \mathrm{H}), 3.39-3.33(\mathrm{~m}, 2 \mathrm{H}), 3.32-3.27(\mathrm{~m}, 2 \mathrm{H}) .{ }^{13} \mathrm{C} \mathrm{NMR}\left(126 \mathrm{MHz}, \mathrm{CDCl}_{3}\right) \delta 159.6$, 156.9, 141.7, 106.9, 104.3, 98.3, 56.3, 55.5, 41.1, 3.0. HRMS (EI): $\mathrm{m} / \mathrm{z}$ calculated for [M, $\left.\mathrm{C}_{10} \mathrm{H}_{12} \mathrm{BrIO}_{2}\right]^{+}:$369.9065; found: 369.9061 . Melting point: $76^{\circ} \mathrm{C}$.

n-BuLi (9.0 mmol, 1.4 equiv) was added to the solution of $i \operatorname{Pr}_{2} \mathrm{NH}$ (11.5 mmol,1.79 equiv) in THF $(15 \mathrm{~mL})$ at $0^{\circ} \mathrm{C}$ under $\mathrm{N}_{2}$ and the mixture was stirred for $30 \mathrm{~min}$. The solution of $\mathrm{N}$ cyclohexylcyclohexanimine (9.0 mmol, 1.4 equiv) in THF $(6 \mathrm{~mL})$ was added dropwise to this mixture and the mixture was stirred at $0^{\circ} \mathrm{C}$ for $1 \mathrm{~h}$. The mixture was cooled to $-78^{\circ} \mathrm{C}$ and the THF $(6 \mathrm{~mL})$ solution of $\mathbf{S 4}\left(6.4 \mathrm{mmol}, 1.0\right.$ equiv.) was added dropwise. After stirring at $-78^{\circ} \mathrm{C}$ for $1 \mathrm{~h}$, saturated aqueous $\mathrm{NH}_{4} \mathrm{Cl}$ solution was added and the mixture was extracted with EtOAc. The combined organic layers were washed with water, brine, dried over anhydrous $\mathrm{Na}_{2} \mathrm{SO}_{4}$, filtered and evaporated under vacuum. Oxalic acid in $\mathrm{H}_{2} \mathrm{O}(1 \mathrm{M}, 9.6 \mathrm{~mL}, 1.5$ equiv.) was added to a solution of the residue in THF $(15 \mathrm{~mL})$ and the mixture was stirred at room temperature overnight. Water was added and the whole was extracted with EtOAc. The combined organic layers were washed with water, brine, dried over anhydrous $\mathrm{Na}_{2} \mathrm{SO}_{4}$, filtered and evaporated under vacuum. The crude product was purified by silica column chromatography to give the desired product 1q-z. 


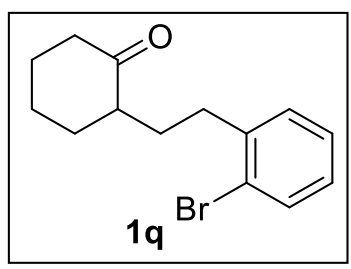

2-Bromophenethyl)cyclohexan-1-one(1q): Yellow oil. (silica gel, eluent, petroleum ether/ethyl acetate $=20: 1) .{ }^{1} \mathrm{H}$ NMR $\left(500 \mathrm{MHz}, \mathrm{CDCl}_{3}\right) \delta 7.52-7.45(\mathrm{~m}, 1 \mathrm{H}), 7.24-7.18(\mathrm{~m}, 2 \mathrm{H}), 7.02(\mathrm{ddd}$, $J=8.1, J=6.6, J=2.6 \mathrm{~Hz}, 1 \mathrm{H}), 2.76-2.71(\mathrm{~m}, 2 \mathrm{H}), 2.44-2.37(\mathrm{~m}, 1 \mathrm{H}), 2.36-2.26(\mathrm{~m}, 2 \mathrm{H}), 2.21-$ $2.13(\mathrm{~m}, 1 \mathrm{H}), 2.12-2.00(\mathrm{~m}, 2 \mathrm{H}), 1.85(\mathrm{~m}, 1 \mathrm{H}), 1.74-1.59(\mathrm{~m}, 2 \mathrm{H}), 1.56-1.39(\mathrm{~m}, 2 \mathrm{H}) .{ }^{13} \mathrm{C}$ NMR $\left(126 \mathrm{MHz}, \mathrm{CDCl}_{3}\right) \delta 213.0,141.6,132.7,130.4,127.5,127.5,124.4,50.0,42.0,34.0,33.5,29.8$, 28.0, 24.9. HRMS (ESI) calculated for $\left[\mathrm{M}+\mathrm{H}, \mathrm{C}_{14} \mathrm{H}_{18} \mathrm{BrO}\right]^{+}:$281.0536; found:281.0537.

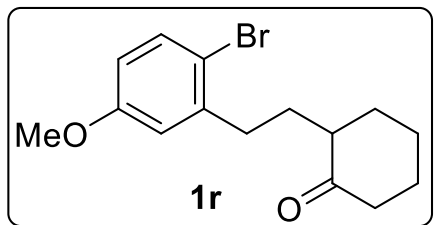

2-Bromo-5-methoxyphenethyl)cyclohexan-1-one(1r): Yellow oil. 82\% yield. (silica gel, eluent, petroleum ether/ethyl acetate $=5: 1) .{ }^{1} \mathrm{H} \mathrm{NMR}\left(500 \mathrm{MHz}, \mathrm{CDCl}_{3}\right) \delta 7.35(\mathrm{~d}, J=8.7 \mathrm{~Hz}, 1 \mathrm{H}), 6.76$ $(\mathrm{d}, J=3.1 \mathrm{~Hz}, 1 \mathrm{H}), 6.59(\mathrm{dd}, J=8.7 \mathrm{~Hz}, J=3.1 \mathrm{~Hz}, 1 \mathrm{H}), 2.71-2.63(\mathrm{~m}, 2 \mathrm{H}), 2.43-2.24(\mathrm{~m}, 3 \mathrm{H})$, $2.20-1.99(\mathrm{~m}, 3 \mathrm{H}), 1.89-1.80(\mathrm{~m}, 1 \mathrm{H}), 1.73-1.60(\mathrm{~m}, 2 \mathrm{H}), 1.55-1.37(\mathrm{~m}, 2 \mathrm{H}) .{ }^{13} \mathrm{C} \mathrm{NMR}(126 \mathrm{MHz}$, $\left.\mathrm{CDCl}_{3}\right) \delta 212.9,158.9,142.5,133.1,115.8,114.8,113.2,55.3,50.0,42.0,34.0,33.7,29.8,28.0$, 24.9. HRMS (ESI) calculated for $\left[\mathrm{M}+\mathrm{H}, \mathrm{C}_{15} \mathrm{H}_{20} \mathrm{BrO}_{2}\right]^{+}: 311.0641$; found: 311.0641 .

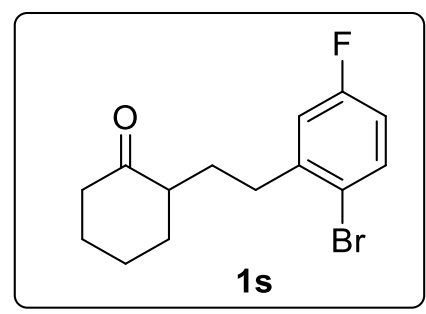

2-Bromo-5-fluorophenethyl)cyclohexan-1-one(1s): Yellow oil. 68\% yield. (silica gel, eluent, petroleum ether/ethyl acetate $=5: 1) .{ }^{1} \mathrm{H} \mathrm{NMR}\left(500 \mathrm{MHz}, \mathrm{CDCl}_{3}\right) \delta 7.45(\mathrm{dd}, J=8.8 \mathrm{~Hz}, J=5.4 \mathrm{~Hz}$, $1 \mathrm{H}), 6.97(\mathrm{dd}, J=9.4 \mathrm{~Hz}, J=3.0 \mathrm{~Hz}, 1 \mathrm{H}), 6.78(\mathrm{td}, J=8.4 \mathrm{~Hz}, J=3.1 \mathrm{~Hz}, 1 \mathrm{H}), 2.72(\mathrm{t}, J=8.1 \mathrm{~Hz}$, $2 \mathrm{H}), 2.45-2.39(\mathrm{~m}, 1 \mathrm{H}), 2.37-2.29(\mathrm{~m}, 2 \mathrm{H}), 2.18(\mathrm{~m}, 1 \mathrm{H}), 2.12-2.03(\mathrm{~m}, 2 \mathrm{H}), 1.92-1.85(\mathrm{~m}$, 1H), $1.73-1.65(\mathrm{~m}, 2 \mathrm{H}), 1.54-1.42(\mathrm{~m}, 2 \mathrm{H}) .{ }^{13} \mathrm{C} \mathrm{NMR}\left(126 \mathrm{MHz}, \mathrm{CDCl}_{3}\right) \delta 212.7,161.5(\mathrm{~d}, J=$ $247.0 \mathrm{~Hz}), 143.8,133.7,118.4,117.1,114.6,50.0,42.1,34.1,33.7,29.6,28.0,25.0 .{ }^{19} \mathrm{~F}$ NMR $(376$ $\left.\mathrm{MHz}, \mathrm{CDCl}_{3}\right) \delta$-115.28. HRMS (ESI) calculated for [M+H, $\left.\mathrm{C}_{14} \mathrm{H}_{17} \mathrm{BrFO}\right]^{+}:$299.0441; found: 299.0442 . 


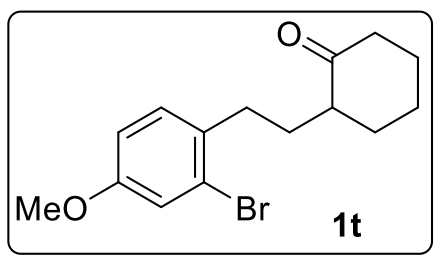

2-Bromo-4-methoxyphenethyl)cyclohexan-1-one(1t): Yellow oil. 54\% yield. (silica gel, eluent, petroleum ether/ethyl acetate $=5: 1) .{ }^{1} \mathrm{H} \mathrm{NMR}\left(500 \mathrm{MHz}, \mathrm{CDCl}_{3}\right) \delta 7.10(\mathrm{~d}, J=8.5 \mathrm{~Hz}, 1 \mathrm{H}), 7.05$ $(\mathrm{d}, J=2.6 \mathrm{~Hz}, 1 \mathrm{H}), 6.76(\mathrm{dd}, J=8.5 \mathrm{~Hz}, J=2.6 \mathrm{~Hz}, 1 \mathrm{H}), 3.74(\mathrm{~s}, 3 \mathrm{H}), 2.65(\mathrm{~m}, 2 \mathrm{H}), 2.38(\mathrm{~m}, 1 \mathrm{H})$, $2.30(\mathrm{~m}, 2 \mathrm{H}), 2.14(\mathrm{~m}, 1 \mathrm{H}), 2.03(\mathrm{~m}, 2 \mathrm{H}), 1.84(\mathrm{~m}, 1 \mathrm{H}), 1.70-1.61(\mathrm{~m}, 2 \mathrm{H}), 1.50-1.39(\mathrm{~m}, 2 \mathrm{H}) .{ }^{13} \mathrm{C}$ $\operatorname{NMR}\left(126 \mathrm{MHz}, \mathrm{CDCl}_{3}\right) \delta 213.0,158.3,133.5,130.6,124.4,117.8,113.6,55.5,50.0,42.1,34.1$, 32.6, 30.1, 28.1, 24.9. HRMS (ESI) calculated for [M+H, $\left.\mathrm{C}_{15} \mathrm{H}_{20} \mathrm{BrO}_{2}\right]^{+}$: 311.0641 ; found: 311.0640 .

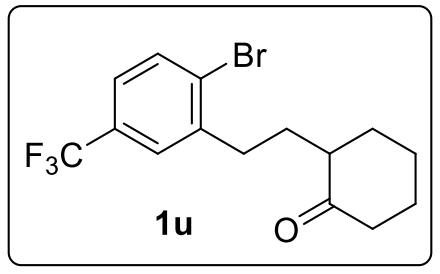

2-Bromo-5-(trifluoromethyl)phenethyl)cyclohexan-1-one(1u): Yellow oil. 62\% yield. (silica gel, eluent, petroleum ether/ethyl acetate $=5: 1) .{ }^{1} \mathrm{H}$ NMR $\left(500 \mathrm{MHz}, \mathrm{CDCl}_{3}\right) \delta 7.61(\mathrm{~d}, J=8.3 \mathrm{~Hz}, 1 \mathrm{H})$, $7.47(\mathrm{~d}, J=2.0 \mathrm{~Hz}, 1 \mathrm{H}), 7.28\left(\mathrm{dd}, J_{1}=8.3, J_{2}=1.9 \mathrm{~Hz}, 1 \mathrm{H}\right), 2.81-2.76(\mathrm{~m}, 2 \mathrm{H}), 2.44-2.38(\mathrm{~m}$, $1 \mathrm{H}), 2.32(\mathrm{~m}, 2 \mathrm{H}), 2.21-2.14(\mathrm{~m}, 1 \mathrm{H}), 2.12-2.03(\mathrm{~m}, 2 \mathrm{H}), 1.92-1.84(\mathrm{~m}, 1 \mathrm{H}), 1.74-1.62(\mathrm{~m}$, 2H), $1.49(\mathrm{~m}, 2 \mathrm{H}) .{ }^{13} \mathrm{C}$ NMR $\left(126 \mathrm{MHz}, \mathrm{CDCl}_{3}\right) \delta 212.6,142.7,133.2,130.0$ (q, $\left.J=32.8 \mathrm{~Hz}\right), 126.8$ $(\mathrm{d}, J=3.8 \mathrm{~Hz}), 124.1$ (d, $J=3.7 \mathrm{~Hz}), 123.8$ (q, $J=277.2 \mathrm{~Hz}), 50.1,42.1,34.1,33.6,29.6,28.0$, 25.0. ${ }^{19} \mathrm{~F}$ NMR $\left(376 \mathrm{MHz}, \mathrm{CDCl}_{3}\right) \delta$-62.66. HRMS (ESI) calculated for $\left[\mathrm{M}+\mathrm{H}, \mathrm{C}_{15} \mathrm{H}_{17} \mathrm{BrF}_{3} \mathrm{O}\right]^{+}$: 349.0409; found: 349.0409 .

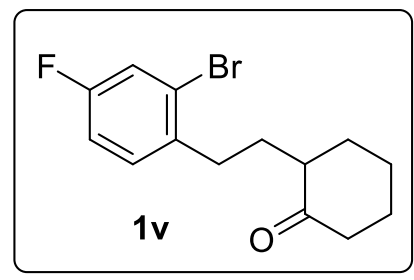

2-(2-Bromo-4-fluorophenethyl)cyclohexan-1-one(1v): Yellow oil. 80\% yield. (silica gel, eluent, petroleum ether/ethyl acetate $=5: 1) .{ }^{1} \mathrm{H}$ NMR $\left(500 \mathrm{MHz}, \mathrm{cdcl}_{3}\right) \delta$ 7.25-7.27 (m, 1H), $7.20(\mathrm{dd}, J=$ $8.5 \mathrm{~Hz}, J=6.1 \mathrm{~Hz}, 1 \mathrm{H}), 6.95(\mathrm{td}, J=8.3 \mathrm{~Hz}, J=2.6 \mathrm{~Hz}, 1 \mathrm{H}), 2.71(\mathrm{t}, J=8.1 \mathrm{~Hz}, 2 \mathrm{H}), 2.42(\mathrm{~m}, 1 \mathrm{H})$, $2.37-2.28(\mathrm{~m}, 2 \mathrm{H}), 2.21-2.14(\mathrm{~m}, 1 \mathrm{H}), 2.06(\mathrm{~m}, 2 \mathrm{H}), 1.90-1.85(\mathrm{~m}, 1 \mathrm{H}), 1.74-1.64(\mathrm{~m}, 2 \mathrm{H})$, $1.52-1.43(\mathrm{~m}, 2 \mathrm{H}) .{ }^{13} \mathrm{C} \mathrm{NMR}\left(126 \mathrm{MHz}, \mathrm{CDCl}_{3}\right) \delta 212.9,160.7(\mathrm{~d}, J=250 \mathrm{~Hz}), 137.4,130.9$, 124.0, 119.7, 114.5, 50.0, 42.1, 34.1, 32.8, 30.0, 28.0, 24.5. HRMS (ESI) calculated for $[\mathrm{M}+\mathrm{H}$, $\left.\mathrm{C}_{14} \mathrm{H}_{17} \mathrm{BrFO}\right]^{+}:$299.0441; found: 299.0441 . 


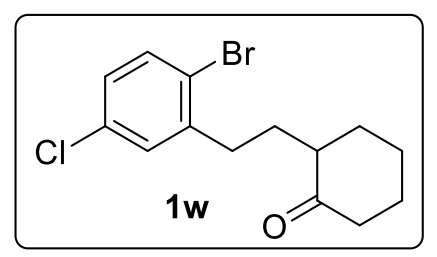

2-Bromo-5-chlorophenethyl)cyclohexan-1-one(1w):Yellow oil. 53\% yield. (silica gel, eluent, petroleum ether/ethyl acetate $=5: 1) .{ }^{1} \mathrm{H}$ NMR $\left(500 \mathrm{MHz}, \mathrm{CDCl}_{3}\right) \delta 7.42(\mathrm{~d}, J=8.5 \mathrm{~Hz}, 1 \mathrm{H}), 7.22$ $(\mathrm{d}, J=2.6 \mathrm{~Hz}, 1 \mathrm{H}), 7.02(\mathrm{dd}, J=8.5 \mathrm{~Hz}, J=2.6 \mathrm{~Hz}, 1 \mathrm{H}), 2.72-2.68(\mathrm{~m}, 2 \mathrm{H}), 2.44-2.39(\mathrm{~m}, 1 \mathrm{H})$, $2.33(\mathrm{~m}, 2 \mathrm{H}), 2.20-2.14(\mathrm{~m}, 1 \mathrm{H}), 2.10-2.02(\mathrm{~m}, 2 \mathrm{H}), 1.91-1.84(\mathrm{~m}, 1 \mathrm{H}), 1.74-1.68(\mathrm{~m}, 1 \mathrm{H})$, $1.66(\mathrm{~m}, 1 \mathrm{H}), 1.53-1.40(\mathrm{~m}, 2 \mathrm{H}) .{ }^{13} \mathrm{C} \mathrm{NMR}\left(126 \mathrm{MHz}, \mathrm{CDCl}_{3}\right) \delta 212.7,143.4,133.6,133.3,130.1$, $127.5,122.1,50.0,42.1,34.1,33.5,29.6,28.0,24.9$. HRMS (ESI) calculated for $[\mathrm{M}+\mathrm{H}$, $\left.\mathrm{C}_{14} \mathrm{H}_{17} \mathrm{BrClO}\right]^{+}:$315.0146; found: 315.0146 .

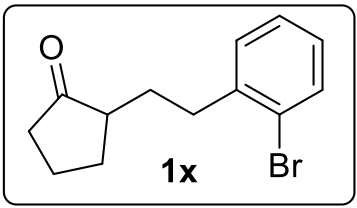

2-Bromophenethyl)cyclopentan-1-one(1x): Yellow oil.58\% yield. (silica gel, eluent, petroleum ether/ethyl acetate $=5: 1) .{ }^{1} \mathrm{H}$ NMR $\left(500 \mathrm{MHz}, \mathrm{CDCl}_{3}\right) \delta 7.51(\mathrm{~d}, J=7.7 \mathrm{~Hz}, 1 \mathrm{H}), 7.25-7.19(\mathrm{~m}$, 2H), $7.04(\mathrm{~m}, 1 \mathrm{H}), 2.86-2.74(\mathrm{~m}, 2 \mathrm{H}), 2.34$ - 2.26 (m, 2H), 2.15 (m, 1H), 2.09 (m, 2H), 2.06 - 1.99 (m, 1H), 1.78-1.66 (m, 1H), 1.66 - $1.56(\mathrm{~m}, 2 \mathrm{H}) .{ }^{13} \mathrm{C} \mathrm{NMR}\left(126 \mathrm{MHz}, \mathrm{CDCl}_{3}\right) \delta 220.9,141.1,132.7$, 130.3, 127.6, 127.4, 124.3, 48.5, 38.1, 33.8,30.0, 29.7, 20.7. HRMS (ESI) calculated for [M+Na, $\left.\mathrm{C}_{13} \mathrm{H}_{15} \mathrm{BrONa}\right]^{+}:$289.0198; found: 289.0198 .

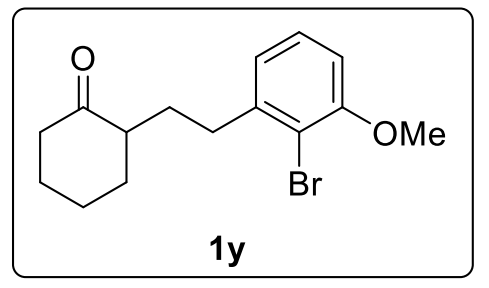

2-Bromo-3-methoxyphenethyl)cyclohexan-1-one(1y): White solid. 94\% yield. (silica gel, eluent, petroleum ether/ethyl acetate $=5: 1) .{ }^{1} \mathrm{H}$ NMR $\left(500 \mathrm{MHz}, \mathrm{CDCl}_{3}\right) \delta 7.18(\mathrm{t}, J=7.9 \mathrm{~Hz}, 1 \mathrm{H}), 6.86$ $(\mathrm{d}, J=7.6 \mathrm{~Hz}, 1 \mathrm{H}), 6.74(\mathrm{~d}, J=8.0 \mathrm{~Hz}, 1 \mathrm{H}), 3.87(\mathrm{~s}, 3 \mathrm{H}), 2.78(\mathrm{~m}, 2 \mathrm{H}), 2.40(\mathrm{~m}, 1 \mathrm{H}), 2.32(\mathrm{~m}, 2 \mathrm{H})$, 2.21 - $2.14(\mathrm{~m}, 1 \mathrm{H}), 2.13$ - $2.00(\mathrm{~m}, 2 \mathrm{H}), 1.92$ - $1.82(\mathrm{~m}, 1 \mathrm{H}), 1.77$ - $1.61(\mathrm{~m}, 2 \mathrm{H}), 1.59$ - $1.39(\mathrm{~m}$, 2H). ${ }^{13} \mathrm{C}$ NMR (126 MHz, $\left.\mathrm{CDCl}_{3}\right) \delta 213.1,155.9,143.4,127.7,122.4,113.8,109.3,56.3,50.0$, 42.0, 34.0, 33.7, 29.7, 28.0, 24.8. HRMS (ESI) calculated for $\left[\mathrm{M}+\mathrm{H}, \mathrm{C}_{15} \mathrm{H}_{20} \mathrm{BrO}_{2}\right]^{+}: 311.0641$; found: 311.0644 . 


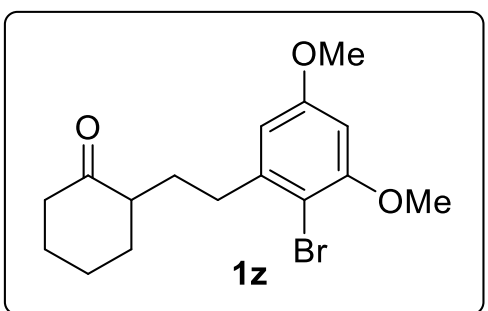

2-Bromo-3,5-dimethoxyphenethyl)cyclohexan-1-one(1z):White solid. 96\% yield. (silica gel, eluent, petroleum ether/ethyl acetate $=5: 1) .{ }^{1} \mathrm{H} \mathrm{NMR}\left(500 \mathrm{MHz}, \mathrm{CDCl}_{3}\right) \delta 6.42(\mathrm{~d}, J=2.7 \mathrm{~Hz}, 1 \mathrm{H})$, $6.34(\mathrm{~d}, J=2.7 \mathrm{~Hz}, 1 \mathrm{H}), 3.85$ (s, 3H), 3.79 (s, 3H), 2.75 (m, 2H), $2.44-2.38(\mathrm{~m}, 1 \mathrm{H}), 2.37-2.28$ (m, 2H), 2.20 - $2.14(\mathrm{~m}, 1 \mathrm{H}), 2.10$ - $2.02(\mathrm{~m}, 2 \mathrm{H}), 1.90$ - $1.83(\mathrm{~m}, 1 \mathrm{H}), 1.72$ - $1.64(\mathrm{~m}, 2 \mathrm{H}), 1.54$ $1.44(\mathrm{~m}, 2 \mathrm{H}) .{ }^{13} \mathrm{C}$ NMR $\left(126 \mathrm{MHz}, \mathrm{CDCl}_{3}\right) \delta$ 213.2, $159.5,156.6,143.6,106.4,104.7,97.5,56.2$, 55.4, 50.1, 42.0, 34.1,34.1, 29.8, 28.1, 24.8. HRMS (ESI) calculated for $\left[\mathrm{M}+\mathrm{H}, \mathrm{C}_{16} \mathrm{H}_{22} \mathrm{BrO}_{3}\right]^{+}$: 341.0747; found: 341.0749 .

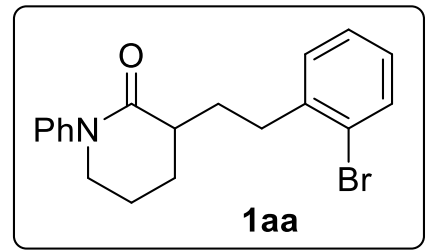

2-Bromophenethyl)-1-phenylpiperidin-2-one(1aa): Brown oil. 51\% yield. (silica gel, eluent, petroleum ether/ethyl acetate $=5: 1) .{ }^{1} \mathrm{H}$ NMR $\left(500 \mathrm{MHz}, \mathrm{CDCl}_{3}\right) \delta 7.52(\mathrm{dd}, J=8.0,1.1 \mathrm{~Hz}, 1 \mathrm{H})$, $7.39(\mathrm{dd}, J=10.4,5.3 \mathrm{~Hz}, 2 \mathrm{H}), 7.32(\mathrm{dd}, J=7.6,1.6 \mathrm{~Hz}, 1 \mathrm{H}), 7.23$ (m, 4H), $7.05(\mathrm{td}, J=7.7,1.7$ $\mathrm{Hz}, 1 \mathrm{H}), 3.73-3.68(\mathrm{~m}, 1 \mathrm{H}), 3.67-3.63(\mathrm{~m}, 1 \mathrm{H}), 2.90(\mathrm{~m}, 2 \mathrm{H}), 2.56(\mathrm{~m}, 1 \mathrm{H}), 2.33-2.24(\mathrm{~m}, 1 \mathrm{H})$, $2.17(\mathrm{~m}, 1 \mathrm{H}), 2.06(\mathrm{~m}, 1 \mathrm{H}), 1.97-1.87(\mathrm{~m}, 2 \mathrm{H}), 1.83(\mathrm{~m}, 1 \mathrm{H}) .{ }^{13} \mathrm{C} \mathrm{NMR}\left(126 \mathrm{MHz}, \mathrm{CDCl}_{3}\right) \delta 172.53$, $143.57,141.45,132.68,130.56,129.07,127.58,127.52$, 126.54, 126.17, 124.41, 51.46, 41.57, 33.74, 32.27, 26.78, 22.20. HRMS (EI) calculated for $\mathrm{C}_{19} \mathrm{H}_{20} \mathrm{NOBr}$, [M] ${ }^{+}$: 357.0728; found: 357.0722 .

\section{General procedures of Pd-catalyzed asymmetric intramolecular $\alpha$-arylation reactions}

To a mixture of compound 1a-z ( $0.2 \mathrm{mmol}, 1.0$ equiv), $\mathrm{NaO} t \mathrm{Bu}\left(0.3 \mathrm{mmol}, 1.5\right.$ equiv), $\mathrm{H}_{2} \mathrm{O}(0.6$ mmol, 3.0 equiv) in THF was charged with $\mathrm{Pd}_{2}(\mathrm{dba})_{3}(0.005 \mathrm{mmol}, 2.5 \mathrm{~mol} \%)$, L9 $(0.012 \mathrm{mmol}$, $6.0 \mathrm{~mol} \%$ ) under $\mathrm{N}_{2}$ atmosphere. The mixture was stirred at $70{ }^{\circ} \mathrm{C}$ (oiling bath) for $24 \mathrm{~h}$ and then cooled to room temperature. The mixture was directly purified by flash chromatography on silica gel to afford the desired product 2a-z. Its enantiomeric excess was determined by chiral HPLC.

\section{Analytical data of the products of Pd-catalyzed asymmetric intramolecular $\alpha$-arylation} reactions 


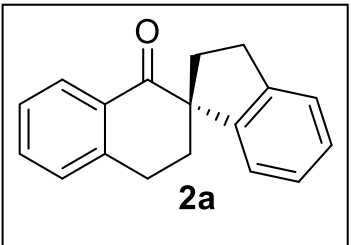

(S)-2,3,3',4'-Tetrahydro-1'H-spiro[indene-1,2'-naphthalen]-1'-one(2a): White solid. 98\% yield, 92\% ee. (silica gel, eluent, petroleum ether/ethyl acetate $=20: 1$ ). Chiral HPLC conditions: chiralcel IC column, $\mathrm{n}$-hexane/ isopropanol $=95: 5$, flow rate $1.0 \mathrm{~mL} / \mathrm{min}, 290 \mathrm{~nm}, t_{1}=10.72 \mathrm{~min}$ (minor), $t_{2}=11.93 \mathrm{~min}$ (major). $[\alpha]_{\mathrm{D}}{ }^{25}=+49.7\left(\mathrm{c}=0.99, \mathrm{CHCl}_{3}\right) .{ }^{1} \mathrm{H} \mathrm{NMR}\left(500 \mathrm{MHz}, \mathrm{CDCl}_{3}\right) \delta 8.13(\mathrm{~d}, J=$ $7.8 \mathrm{~Hz}, 1 \mathrm{H}), 7.54(\mathrm{td}, J=7.5 \mathrm{~Hz}, J=0.9 \mathrm{~Hz}, 1 \mathrm{H}), 7.37$ (t, $J=7.5 \mathrm{~Hz}, 1 \mathrm{H}), 7.34-7.30(\mathrm{~m}, 2 \mathrm{H}), 7.24$ (t, $J=7.8 \mathrm{~Hz}, 1 \mathrm{H}), 7.15(\mathrm{t}, J=7.4 \mathrm{~Hz}, 1 \mathrm{H}), 6.99(\mathrm{~d}, J=7.6 \mathrm{~Hz}, 1 \mathrm{H}), 3.16-3.03(\mathrm{~m}, 4 \mathrm{H}), 2.78$ $2.70(\mathrm{~m}, 1 \mathrm{H}), 2.48(\mathrm{~m}, 1 \mathrm{H}), 2.21(\mathrm{~m}, 1 \mathrm{H}), 2.16-2.10(\mathrm{~m}, 1 \mathrm{H}) .{ }^{13} \mathrm{C} \mathrm{NMR}\left(126 \mathrm{MHz}, \mathrm{CDCl}_{3}\right) \delta 199.5$, 145.4, 144.3, 143.8, 133.3, 132.2, 128.8, 128.3, 127.5, 126.8, 126.3, 124.9, 123.6, 58.9, 35.1, 34.0, 30.2, 26.2. HRMS (ESI) calculated for $\left[\mathrm{M}+\mathrm{H}, \mathrm{C}_{18} \mathrm{H}_{17} \mathrm{O}\right]^{+}: 249.1274$; found: 249.1277 . Melting point: $78^{\circ} \mathrm{C}$.
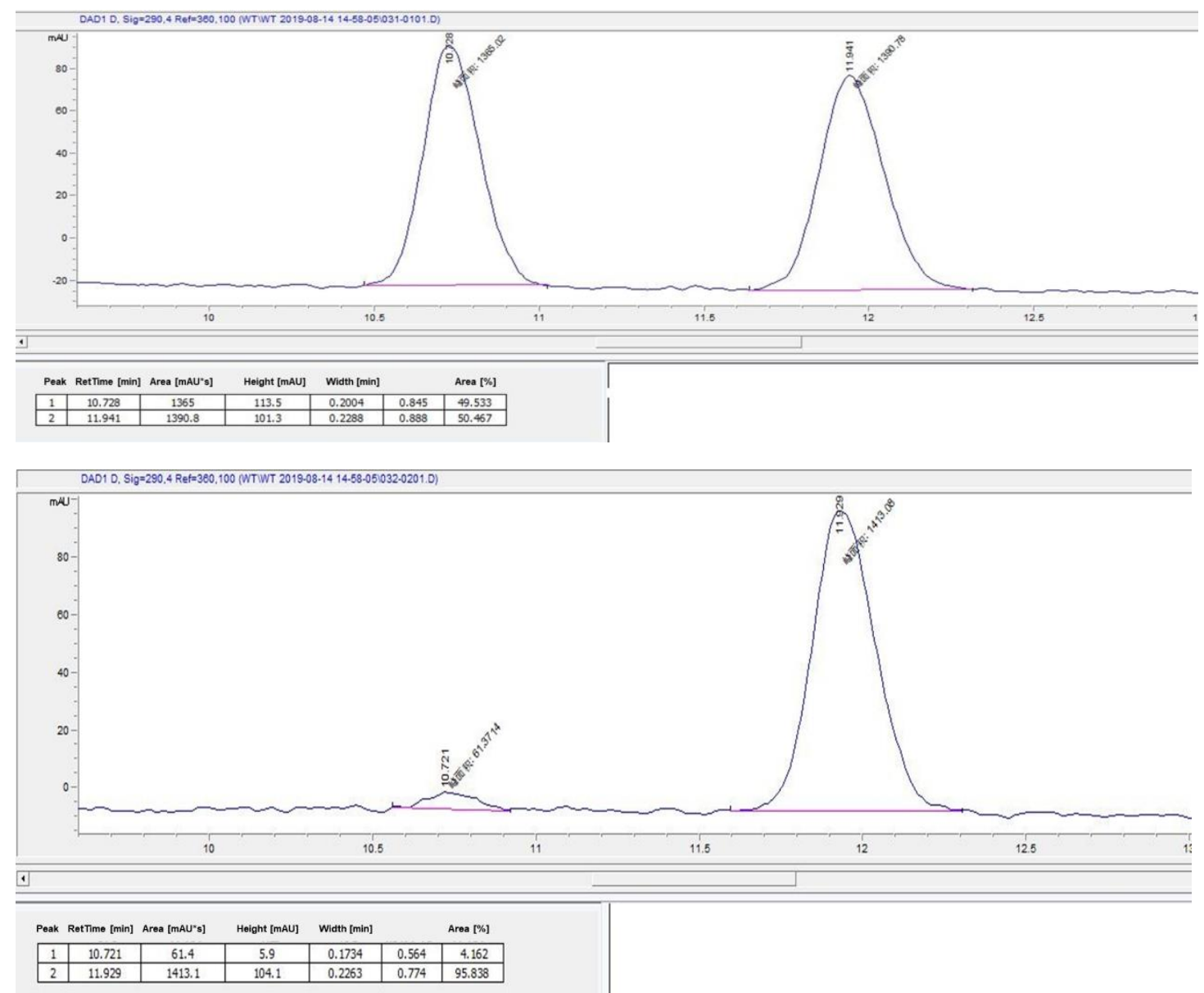


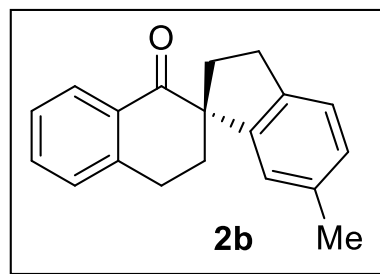

(S)-6-Methyl-2,3,3',4'-tetrahydro-1'H-spiro[indene-1,2'-naphthalen]-1'-one(2b): White solid. ( silica gel, eluent, petroleum ether/ethyl acetate $=20: 1$ ). $77 \%$ yield, $84 \%$ ee. Chiral HPLC conditions: chiralcel IC column, $\mathrm{n}$-hexane/ isopropanol $=95: 5$, flow rate $1.0 \mathrm{~mL} / \mathrm{min}, 290 \mathrm{~nm}, t_{1}=9.03 \mathrm{~min}$ (minor), $t_{2}=10.81 \mathrm{~min}$ (major). $[\alpha]_{\mathrm{D}}{ }^{25}=+7.6\left(\mathrm{c}=1.00, \mathrm{CHCl}_{3}\right) .{ }^{1} \mathrm{H} \mathrm{NMR}\left(500 \mathrm{MHz}, \mathrm{CDCl}_{3}\right) \delta 8.10$ $(\mathrm{dd}, J=7.9 \mathrm{~Hz}, J=1.0 \mathrm{~Hz}, 1 \mathrm{H}), 7.52(\mathrm{td}, J=7.5 \mathrm{~Hz}, J=1.4 \mathrm{~Hz}, 1 \mathrm{H}), 7.38-7.33(\mathrm{~m}, 1 \mathrm{H}), 7.31(\mathrm{~s}$, 1H), $7.17(\mathrm{~d}, J=7.7 \mathrm{~Hz}, 1 \mathrm{H}), 7.03(\mathrm{dd}, J=7.7 \mathrm{~Hz}, J=0.7 \mathrm{~Hz}, 1 \mathrm{H}), 6.79(\mathrm{~s}, 1 \mathrm{H}), 3.13-3.05(\mathrm{~m}$, 2H), $2.98(\mathrm{~m}, 2 \mathrm{H}), 2.65(\mathrm{~m}, 1 \mathrm{H}), 2.51-2.44(\mathrm{~m}, 1 \mathrm{H}), 2.27(\mathrm{~s}, 3 \mathrm{H}), 2.20-2.09(\mathrm{~m}, 2 \mathrm{H}) .{ }^{13} \mathrm{C}$ NMR $\left(126 \mathrm{MHz}, \mathrm{CDCl}_{3}\right) \delta 199.9,145.7,143.8,141.3,135.9,133.3,132.1,128.8,128.4,128.2,126.8$, 124.5, 124.1, 58.9, 35.4, 34.1, 29.8,26.3, 21.4. HRMS (ESI) calculated for $\left[\mathrm{M}+\mathrm{H}, \mathrm{C}_{19} \mathrm{H}_{19} \mathrm{O}\right]^{+}$: 263.1430; found: 263.1433 .
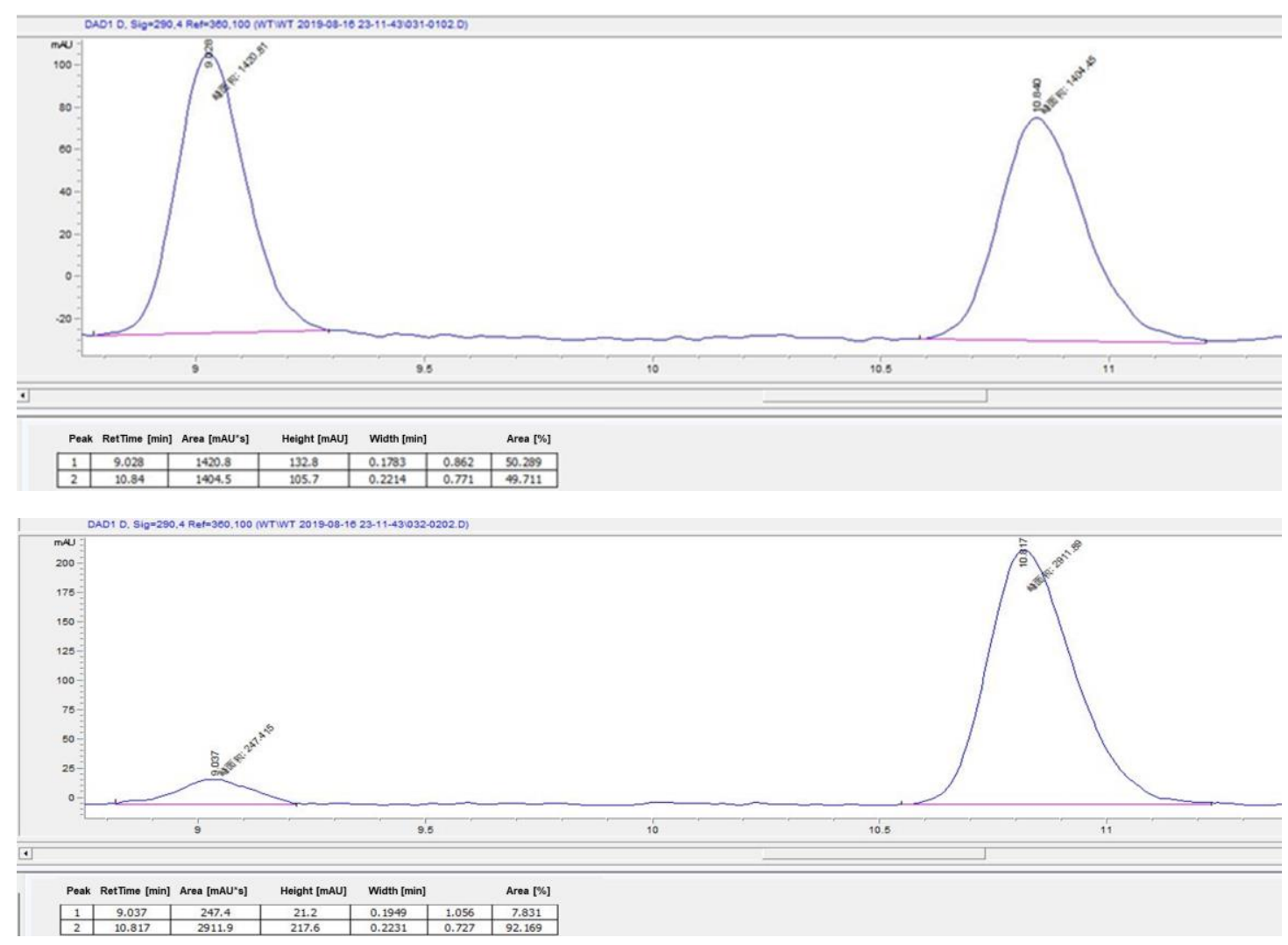


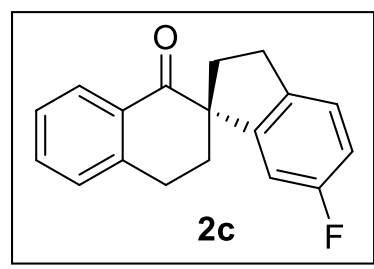

(S)-6-Fluoro-2,3,3',4'-tetrahydro-1'H-spiro[indene-1,2'-naphthalen]-1'-one(2c): White solid. $90 \%$ yield, $86 \%$ ee. (silica gel, eluent, petroleum ether/ethyl acetate $=20: 1$ ). Chiral HPLC conditions: chiralcel IC column, $\mathrm{n}$-hexane/ isopropanol $=95: 5$, flow rate $1.0 \mathrm{~mL} / \mathrm{min}, 290 \mathrm{~nm}, t_{1}=$ $8.26 \min$ (minor), $t_{2}=9.15 \min$ (major). $[\alpha]_{\mathrm{D}}{ }^{25}=+38.5\left(\mathrm{c}=1.06, \mathrm{CHCl}_{3}\right){ }^{1} \mathrm{H}$ NMR $(500 \mathrm{MHz}$, $\left.\mathrm{CDCl}_{3}\right) \delta 8.09(\mathrm{~d}, J=7.9 \mathrm{~Hz}, 1 \mathrm{H}), 7.53(\mathrm{td}, J=7.5, J=1.4 \mathrm{~Hz}, 1 \mathrm{H}), 7.36(\mathrm{t}, J=7.6 \mathrm{~Hz}, 1 \mathrm{H}), 7.30(\mathrm{~d}$, $J=7.7 \mathrm{~Hz}, 1 \mathrm{H}), 7.21(\mathrm{dd}, J=8.2, J=5.2 \mathrm{~Hz}, 1 \mathrm{H}), 6.91(\mathrm{td}, J=8.7, J=2.5 \mathrm{~Hz}, 1 \mathrm{H}), 6.67(\mathrm{dd}, J=$ 9.0, $J=2.3 \mathrm{~Hz}, 1 \mathrm{H}), 3.14-3.05(\mathrm{~m}, 2 \mathrm{H}), 2.97$ (dd, $J=13.8, J=6.7 \mathrm{~Hz}, 2 \mathrm{H}), 2.69(\mathrm{~m}, 1 \mathrm{H}), 2.45(\mathrm{~m}$, 1H), 2.24 - $2.12(\mathrm{~m}, 2 \mathrm{H}) ;{ }^{13} \mathrm{C} \mathrm{NMR}\left(126 \mathrm{MHz}, \mathrm{CDCl}_{3}\right) \delta 198.9,177.4,161.9(\mathrm{~J}=247.0 \mathrm{~Hz}), 143.5$, 139.6, 133.5, 131.8, 128.8, 128.2, 126.9, 125.6, 114.3, 110.7, 58.9, 35.5, 33.8, 29.4, 26.0. ${ }^{19} \mathrm{~F}$ NMR $\left(376 \mathrm{MHz}, \mathrm{CDCl}_{3}\right) \delta-116.96$. HRMS (ESI) calculated for $\left[\mathrm{M}+\mathrm{H}, \mathrm{C}_{18} \mathrm{H}_{16} \mathrm{FO}\right]^{+}: 267.1180$; found: 267.1180 . 

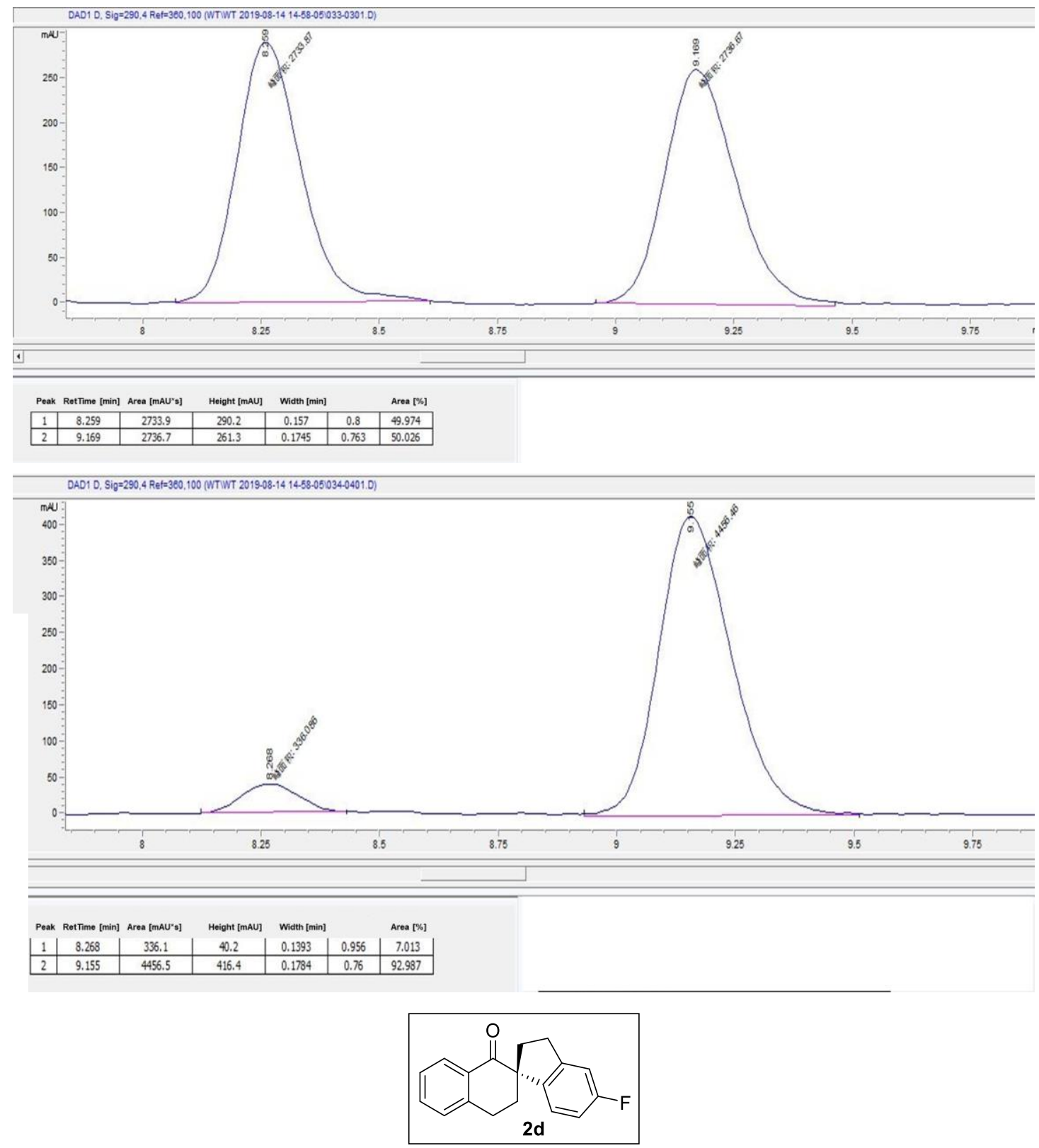

(S)-5-Fluoro-2,3,3',4'-tetrahydro-1'H-spiro[indene-1,2'-naphthalen]-1'-one(2d): White solid. $75 \%$ yield, $90 \%$ ee. (silica gel, eluent, petroleum ether/ethyl acetate $=20: 1$ ). Chiral HPLC conditions chiralcel IC column, $\mathrm{n}$-hexane/ isopropanol $=97: 3$, flow rate $1.0 \mathrm{~mL} / \mathrm{min}, 290 \mathrm{~nm}, t_{1}=11.08$ $\min$ (major), $t_{2}=11.72 \min$ (minor). $[\alpha]_{\mathrm{D}}{ }^{25}=+54.6\left(\mathrm{c}=0.47, \mathrm{CHCl}_{3}\right) .{ }^{1} \mathrm{H} \mathrm{NMR}\left(500 \mathrm{MHz}, \mathrm{CDCl}_{3}\right) \delta$ 8.09 (d, $J=7.8 \mathrm{~Hz}, 1 \mathrm{H}), 7.52$ (td, $J=7.5 \mathrm{~Hz}, J=1.3 \mathrm{~Hz}, 1 \mathrm{H}), 7.35(\mathrm{t}, J=7.5 \mathrm{~Hz}, 1 \mathrm{H}), 7.30$ (d, $J=$ $7.7 \mathrm{~Hz}, 1 \mathrm{H}), 6.97(\mathrm{dd}, J=8.9 \mathrm{~Hz}, J=2.2 \mathrm{~Hz}, 1 \mathrm{H}), 6.90(\mathrm{dd}, J=8.4 \mathrm{~Hz}, J=5.2 \mathrm{~Hz}, 1 \mathrm{H}), 6.82(\mathrm{td}, J=$ $8.7 \mathrm{~Hz}, J=2.4 \mathrm{~Hz}, 1 \mathrm{H}), 3.10(\mathrm{~m}, 2 \mathrm{H}), 3.01(\mathrm{~m}, 2 \mathrm{H}), 2.69(\mathrm{~m}, 1 \mathrm{H}), 2.45(\mathrm{~m}, 1 \mathrm{H}), 2.20-2.12(\mathrm{~m}, 2 \mathrm{H})$. ${ }^{13} \mathrm{C}$ NMR $\left(126 \mathrm{MHz}, \mathrm{CDCl}_{3}\right) \delta 199.3,163.6(\mathrm{~J}=244 \mathrm{~Hz}), 146.6,143.6,141.0,133.4,132.0,128.8$, $128.3,126.9,124.5,113.3,112.0,58.1,35.3,34.1,30.2,26.2 .{ }^{19} \mathrm{~F} \mathrm{NMR}\left(376 \mathrm{MHz}, \mathrm{CDCl}_{3}\right) \delta-$ 115.98. HRMS (ESI) calculated for [M+H, $\left.\mathrm{C}_{18} \mathrm{H}_{16} \mathrm{FO}\right]^{+}: 267.1180$; found: 267.1181. 

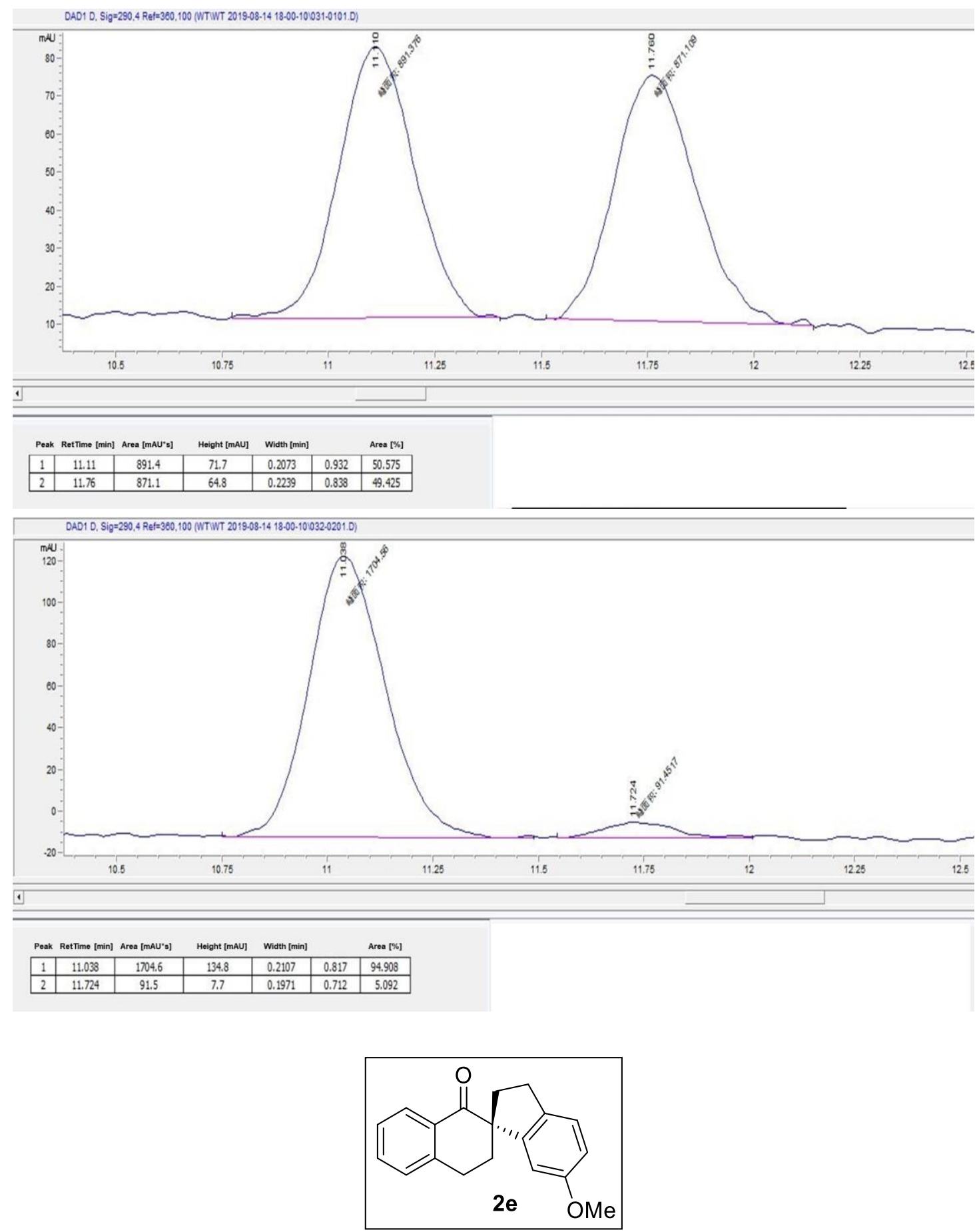

(S)-6-Methoxy-2,3,3',4'-tetrahydro-1'H-spiro[indene-1,2'-naphthalen]-1'-one(2e): $\quad$ Yellow solid. $89 \%$ yield, $88 \%$ ee. (silica gel, eluent, petroleum ether/ethyl acetate $=20: 1$ ). Chiral HPLC conditions: chiralcel IC column, $\mathrm{n}$-hexane/ isopropanol $=95: 5$, flow rate $1.0 \mathrm{~mL} / \mathrm{min}, 290 \mathrm{~nm}, t_{1}=$ $9.95 \mathrm{~min}$ (minor), $t_{2}=11.70 \mathrm{~min}$ (major). $[\alpha]_{\mathrm{D}}{ }^{25}=+28.6\left(\mathrm{c}=1.24, \mathrm{CHCl}_{3}\right) .{ }^{1} \mathrm{H} \mathrm{NMR}(500 \mathrm{MHz}$, $\left.\mathrm{CDCl}_{3}\right) \delta 8.10(\mathrm{~d}, J=7.8 \mathrm{~Hz}, 1 \mathrm{H}), 7.51(\mathrm{t}, J=7.3 \mathrm{~Hz}, 1 \mathrm{H}), 7.35(\mathrm{t}, J=7.5 \mathrm{~Hz}, 1 \mathrm{H}), 7.30(\mathrm{~d}, J=7.7$ 
$\mathrm{Hz}, 1 \mathrm{H}), 7.18(\mathrm{~d}, J=8.3 \mathrm{~Hz}, 1 \mathrm{H}), 6.79-6.75(\mathrm{~m}, 1 \mathrm{H}), 6.54(\mathrm{~d}, J=2.2 \mathrm{~Hz}, 1 \mathrm{H}), 3.71(\mathrm{~s}, 3 \mathrm{H}), 3.10$ (m, 2H), $2.98-2.93(\mathrm{~m}, 2 \mathrm{H}), 2.71-2.65(\mathrm{~m}, 1 \mathrm{H}), 2.47(\mathrm{~m}, 1 \mathrm{H}), 2.21-2.10(\mathrm{~m}, 2 \mathrm{H}) .{ }^{13} \mathrm{C}$ NMR $(126$ $\left.\mathrm{MHz}, \mathrm{CDCl}_{3}\right) \delta 199.4,158.5,146.9,143.7,136.3,133.4,132.1,128.8,128.3,126.8,125.2,113.0$, 109.6, 59.0, 55.4, 35.6, 34.0, 29.4, 26.2. HRMS (ESI) calculated for $\left[\mathrm{M}+\mathrm{H}, \mathrm{C}_{19} \mathrm{H}_{19} \mathrm{O}_{2}\right]^{+}$: 279.1380; found: 279.1382 .
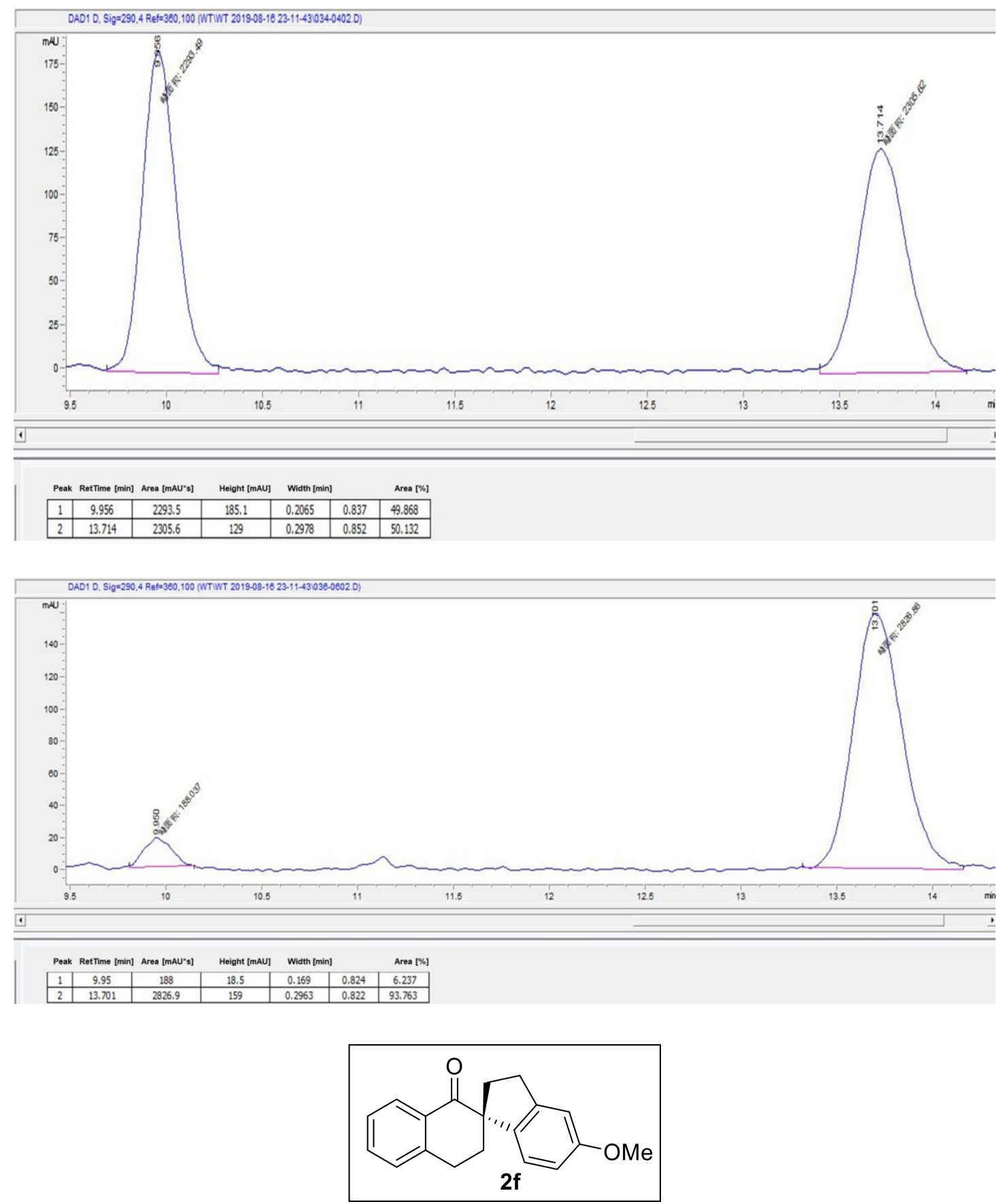
(S)-5-Methoxy-2,3,3',4'-tetrahydro-1'H-spiro[indene-1,2'-naphthalen]-1'-one (2f): White solid. $97 \%$ yield, $90 \%$ ee. ( silica gel, eluent, petroleum ether/ethyl acetate $=20: 1$ ). Chiral HPLC conditions: chiralcel IC column, $\mathrm{n}$-hexane/ isopropanol $=95: 5$, flow rate $1.0 \mathrm{~mL} / \mathrm{min}, 290 \mathrm{~nm}, t_{1}=13.42 \mathrm{~min}$ (major), $t_{2}=18.45 \mathrm{~min}$ (minor). $[\alpha]_{\mathrm{D}}{ }^{25}=+49.0\left(\mathrm{c}=1.08, \mathrm{CHCl}_{3}\right){ }^{1} \mathrm{H} \mathrm{NMR}\left(500 \mathrm{MHz}, \mathrm{CDCl}_{3}\right) \delta$ $8.09(\mathrm{dd}, J=7.8 \mathrm{~Hz}, J=1.0 \mathrm{~Hz}, 1 \mathrm{H}), 7.51(\mathrm{td}, J=7.5 \mathrm{~Hz}, J=1.4 \mathrm{~Hz}, 1 \mathrm{H}), 7.35(\mathrm{dd}, J=11.2 \mathrm{~Hz}, J$ $=3.9 \mathrm{~Hz}, 1 \mathrm{H}), 7.30(\mathrm{~d}, J=7.6 \mathrm{~Hz}, 1 \mathrm{H}), 6.85(\mathrm{dd}, J=13.5 \mathrm{~Hz}, J=5.3 \mathrm{~Hz}, 2 \mathrm{H}), 6.67(\mathrm{dd}, J=8.4$, $2.5 \mathrm{~Hz}, 1 \mathrm{H}), 3.78$ (s, 3H), $3.08(\mathrm{t}, J=6.2 \mathrm{~Hz}, 2 \mathrm{H}), 3.03-2.97(\mathrm{~m}, 2 \mathrm{H}), 2.71(\mathrm{~m}, 1 \mathrm{H}), 2.46-2.40$ (m, 1H), $2.17(\mathrm{~m}, 1 \mathrm{H}), 2.12-2.07(\mathrm{~m}, 1 \mathrm{H}) .{ }^{13} \mathrm{C} \mathrm{NMR}\left(126 \mathrm{MHz}, \mathrm{CDCl}_{3}\right) \delta$ 199.7, 159.5, 146.0, $143.8,137.5,133.3,132.2,128.8,128.2,126.7,124.1,112.3,110.2,58.1,55.3,35.4,34.2,30.4$, 26.3. HRMS (ESI) calculated for $\left[\mathrm{M}+\mathrm{H}, \mathrm{C}_{19} \mathrm{H}_{19} \mathrm{O}_{2}\right]^{+}: 279.1380$; found: 279.1380 .
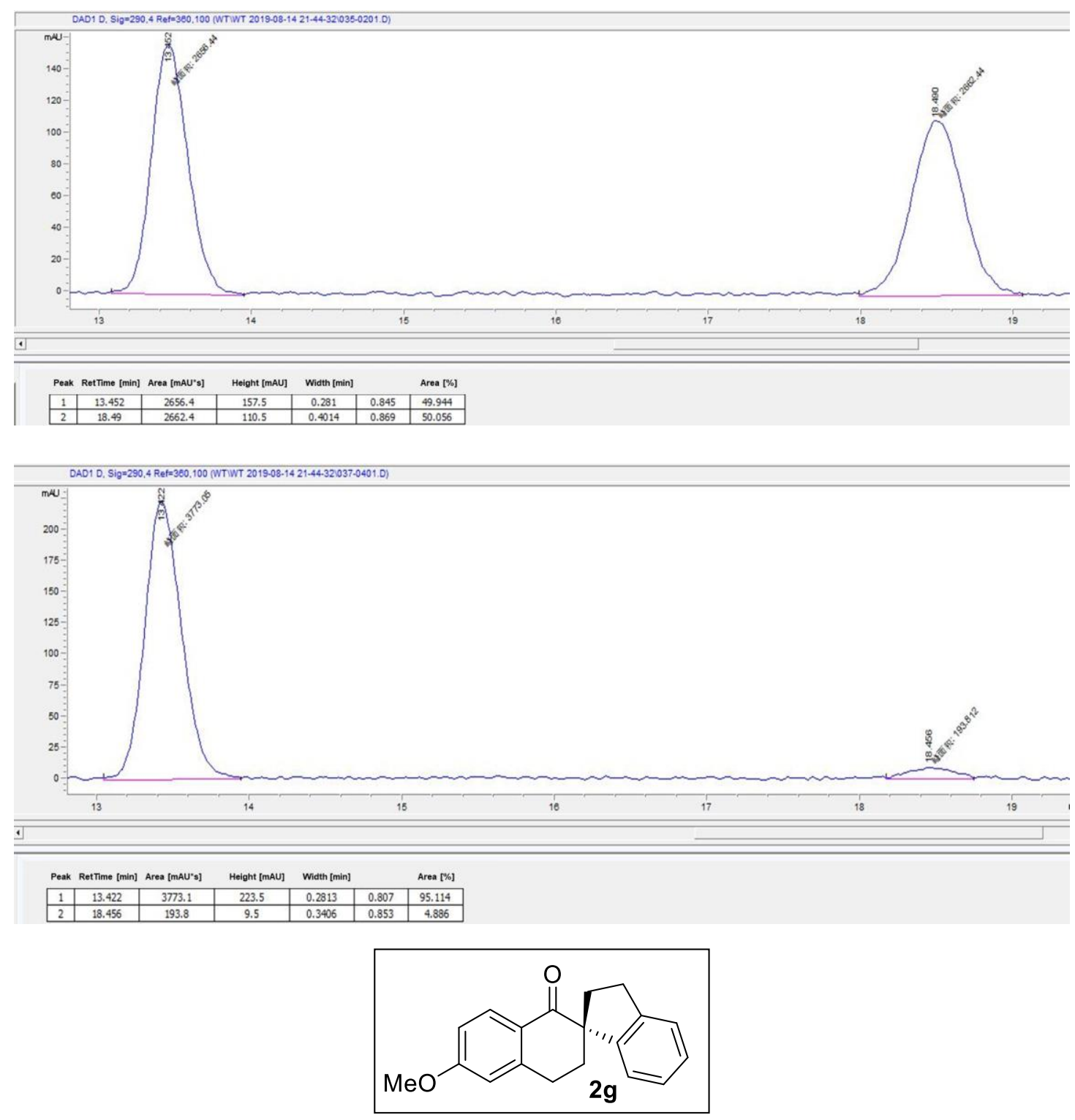
(S)-6'-Methoxy-2,3,3',4'-tetrahydro-1'H-spiro[indene-1,2'-naphthalen]-1'-one(2g): $\quad$ White solid. $98 \%$ yield, $92 \%$ ee. (silica gel, eluent, petroleum ether/ethyl acetate $=20: 1$ ). Chiral HPLC conditions: chiralcel IC column, n-hexane/ isopropanol = 95: 5, flow rate $1.0 \mathrm{~mL} / \mathrm{min}, 290 \mathrm{~nm}, t_{1}=$ 24.66min (minor), $t_{2}=30.27 \mathrm{~min}$ (major). $[\alpha]_{\mathrm{D}}{ }^{25}=+79.5\left(\mathrm{c}=1.07, \mathrm{CHCl}_{3}\right) .{ }^{1} \mathrm{H} \mathrm{NMR}(500 \mathrm{MHz}$, $\left.\mathrm{CDCl}_{3}\right) \delta 7.58(\mathrm{~d}, J=2.8 \mathrm{~Hz}, 1 \mathrm{H}), 7.29(\mathrm{~d}, J=7.5 \mathrm{~Hz}, 1 \mathrm{H}), 7.23-7.19(\mathrm{~m}, 2 \mathrm{H}), 7.15-7.09(\mathrm{~m}$, 2H), $6.96(\mathrm{~d}, J=7.6 \mathrm{~Hz}, 1 \mathrm{H}), 3.85$ (s, 3H), $3.05-2.98(\mathrm{~m}, 4 \mathrm{H}), 2.72(\mathrm{~m}, 1 \mathrm{H}), 2.43(\mathrm{~m}, 1 \mathrm{H}), 2.17$ (m, 1H), $2.10(\mathrm{~m}, 1 \mathrm{H}) .{ }^{13} \mathrm{C}$ NMR $\left(126 \mathrm{MHz}, \mathrm{CDCl}_{3}\right) \delta 199.5,158.4,145.3,144.2,136.41,132.9$, 130.0, 127.4, 126.3, 124.8, 123.6, 121.8, 110.0, 58.7, 55.4, 35.0, 34.2, 30.2, 25.4.HRMS (ESI) calculated for $\left[\mathrm{M}+\mathrm{H}, \mathrm{C}_{19} \mathrm{H}_{19} \mathrm{O}_{2}\right]^{+}: 279.1380$; found: 279.1384 .

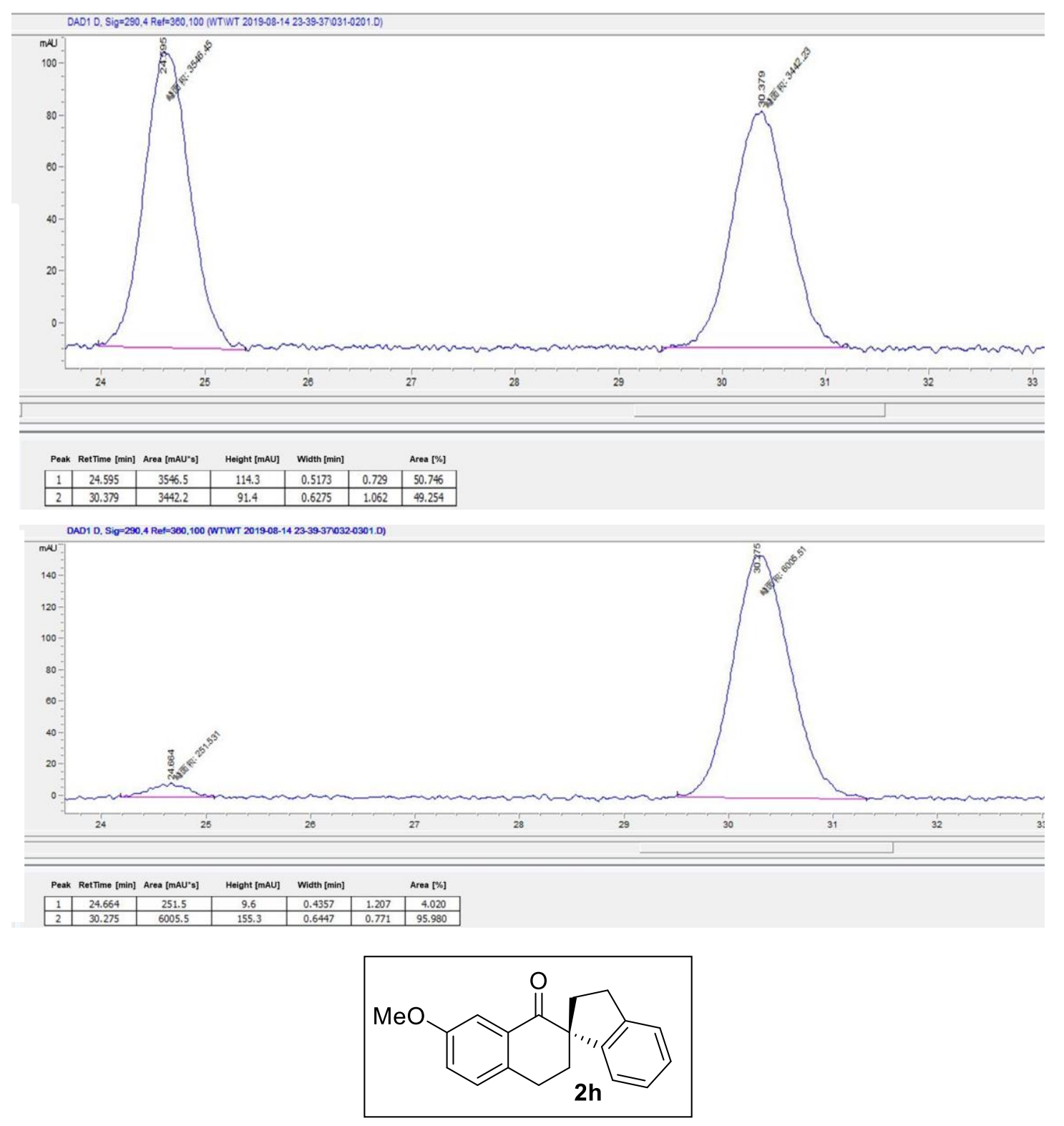

(S)-7'-Methoxy-2,3,3',4'-tetrahydro-1'H-spiro[indene-1,2'-naphthalen]-1'-one(2h): $\quad$ White solid. 94\% yield, 93\% ee. (silica gel, eluent, petroleum ether/ethyl acetate $=20: 1$ ). Chiral HPLC 
conditions: chiralcel S-Chiral B column, $\mathrm{n}$-hexane/ isopropanol = 95: 5, flow rate $1.0 \mathrm{~mL} / \mathrm{min}, 290$ $\mathrm{nm}, t_{1}=10.16 \mathrm{~min}$ (minor), $t_{2}=15.00 \mathrm{~min}$ (major). $[\alpha]_{\mathrm{D}}{ }^{25}=+22.3\left(\mathrm{c}=1.00, \mathrm{CHCl}_{3}\right) .{ }^{1} \mathrm{H}$ NMR $(500$ $\left.\mathrm{MHz}, \mathrm{CDCl}_{3}\right) \delta 8.08(\mathrm{~d}, J=8.8 \mathrm{~Hz}, 1 \mathrm{H}), 7.30-7.25(\mathrm{~m}, 1 \mathrm{H}), 7.20(\mathrm{td}, J=7.4 \mathrm{~Hz}, J=1.0 \mathrm{~Hz}, 1 \mathrm{H})$, $7.11(\mathrm{t}, J=7.4 \mathrm{~Hz}, 1 \mathrm{H}), 6.96(\mathrm{~d}, J=7.6 \mathrm{~Hz}, 1 \mathrm{H}), 6.88(\mathrm{dd}, J=8.8 \mathrm{~Hz}, J=2.5 \mathrm{~Hz}, 1 \mathrm{H}), 6.75(\mathrm{~d}, J=$ $2.4 \mathrm{~Hz}, 1 \mathrm{H}), 3.89$ (s, 3H), $3.04(\mathrm{dd}, J=11.2 \mathrm{~Hz}, J=5.3 \mathrm{~Hz}, 4 \mathrm{H}), 2.77-2.70(\mathrm{~m}, 1 \mathrm{H}), 2.42(\mathrm{~m}, 1 \mathrm{H})$, $2.17(\mathrm{~m}, 1 \mathrm{H}), 2.10-2.05(\mathrm{~m}, 1 \mathrm{H}) .{ }^{13} \mathrm{C} \mathrm{NMR}\left(126 \mathrm{MHz}, \mathrm{CDCl}_{3}\right) \delta$ 199.5, 158.4, 145.5, 144.3, 136.4, 133.0, 130.0, 127.5, 126.3, 124.9, 123.6, 121.8, 110.0, 58.7, 55.5, 35.0, 34.2, 30.2, 25.4. HRMS ESI) calculated for $\left[\mathrm{M}+\mathrm{H}, \mathrm{C}_{19} \mathrm{H}_{19} \mathrm{O}_{2}\right]^{+}:$279.1380; found: 279.1381 .

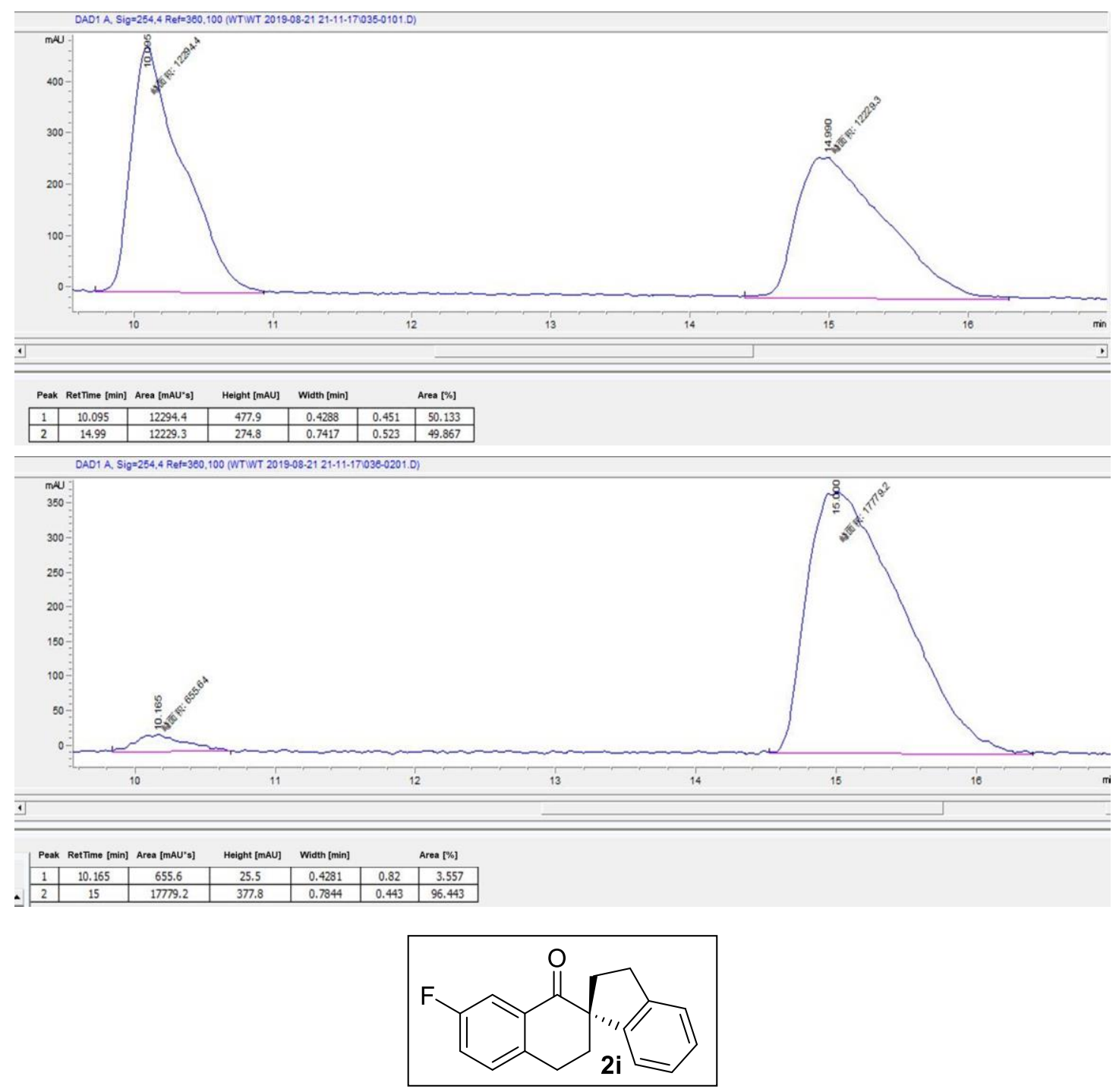

(S)-7'-Fluoro-2,3,3',4'-tetrahydro-1'H-spiro[indene-1,2'-naphthalen]-1'-one(2i): White solid. $89 \%$ yield, $94 \%$ ee. (silica gel, eluent, petroleum ether/ethyl acetate $=20: 1$ ). Chiral HPLC conditions: chiralcel IC column, $\mathrm{n}$-hexane/ isopropanol $=95: 5$, flow rate $1.0 \mathrm{~mL} / \mathrm{min}, 254 \mathrm{~nm}, t_{1}=8.52 \mathrm{~min}$ 
(major), $t_{2}=9.03 \mathrm{~min}$ (minor). $[\alpha]_{\mathrm{D}}{ }^{25}=+110.7\left(\mathrm{c}=0.40, \mathrm{CHCl}_{3}\right) .{ }^{1} \mathrm{H} \mathrm{NMR}\left(500 \mathrm{MHz}, \mathrm{cdcl}_{3}\right) \delta 7.76$ $(\mathrm{dd}, J=9.2 \mathrm{~Hz}, J=2.8 \mathrm{~Hz}, 1 \mathrm{H}), 7.29(\mathrm{dd}, J=7.8 \mathrm{~Hz}, J=3.4 \mathrm{~Hz}, 2 \mathrm{H}), 7.25(\mathrm{dd}, J=7.9 \mathrm{~Hz}, J=4.5$ $\mathrm{Hz}, 1 \mathrm{H}), 7.22(\mathrm{~d}, J=2.8 \mathrm{~Hz}, 1 \mathrm{H}), 7.13(\mathrm{t}, J=7.4 \mathrm{~Hz}, 1 \mathrm{H}), 6.96(\mathrm{~d}, J=7.6 \mathrm{~Hz}, 1 \mathrm{H}), 3.04(\mathrm{dt}, J=$ $7.5, J=4.5 \mathrm{~Hz}, 5 \mathrm{H}), 2.74-2.66(\mathrm{~m}, 1 \mathrm{H}), 2.50-2.42(\mathrm{~m}, 1 \mathrm{H}), 2.19(\mathrm{~m}, 1 \mathrm{H}), 2.13-2.07(\mathrm{~m}, 1 \mathrm{H})$. ${ }^{13} \mathrm{C} \mathrm{NMR}\left(126 \mathrm{MHz}, \mathrm{CDCl}_{3}\right) \delta 198.5,161.7(\mathrm{~J}=252 \mathrm{~Hz}), 145.0,144.3,139.5,138.8,130.6,127.6$, 126.4, 124.9, 123.5, 120.8, 114.0, 58.6, 35.0, 34.0, 30.2, 25.5. ${ }^{19} \mathrm{~F}$ NMR $\left(376 \mathrm{MHz}, \mathrm{CDCl}_{3}\right) \delta-$ 115.88. HRMS (ESI) calculated for $\left[\mathrm{M}+\mathrm{H}, \mathrm{C}_{18} \mathrm{H}_{16} \mathrm{FO}\right]^{+}: 267.1180$; found: 267.1178.
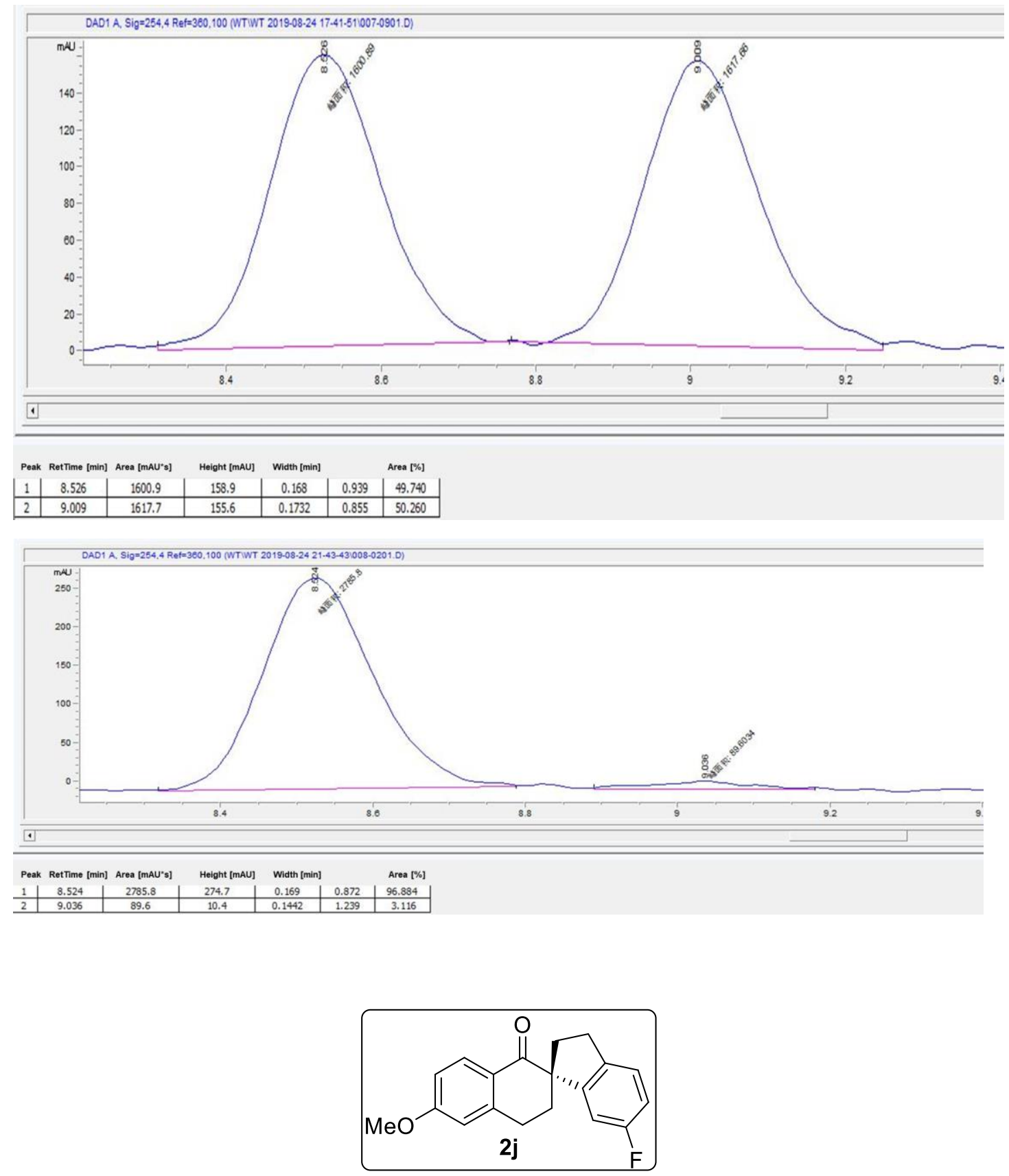


\section{(S)-6-Fluoro-6'-methoxy-2,3,3',4'-tetrahydro-1'H-spiro[indene-1,2'-naphthalen]-1'-one (2j):}

Yellow solid. $67 \%$ yield, $84 \%$ ee. (silica gel, eluent, petroleum ether/ethyl acetate $=20: 1$ ). Chiral HPLC conditions: chiralcel IC column, $\mathrm{n}$-hexane/ isopropanol $=95: 5$, flow rate $1.0 \mathrm{~mL} / \mathrm{min}, 290$ $\mathrm{nm}, t_{1}=16.02 \mathrm{~min}$ (minor), $t_{2}=18.83 \mathrm{~min}$ (major). $[\alpha]_{\mathrm{D}}{ }^{25}=+56.9\left(\mathrm{c}=1.02, \mathrm{CHCl}_{3}\right) .{ }^{1} \mathrm{H} \mathrm{NMR}(500$ $\left.\mathrm{MHz}, \mathrm{CDCl}_{3}\right) \delta 7.56(\mathrm{~d}, J=2.8 \mathrm{~Hz}, 1 \mathrm{H}), 7.21(\mathrm{~d}, J=8.5 \mathrm{~Hz}, 1 \mathrm{H}), 7.11(\mathrm{dd}, J=8.4 \mathrm{~Hz}, J=2.8 \mathrm{~Hz}$, $1 \mathrm{H}), 6.98-6.95(\mathrm{~m}, 1 \mathrm{H}), 6.90(\mathrm{dd}, J=8.4 \mathrm{~Hz}, J=5.2 \mathrm{~Hz}, 1 \mathrm{H}), 6.81(\mathrm{td}, J=8.7 \mathrm{~Hz}, \quad J=2.4 \mathrm{~Hz}$, 1H), $3.84(\mathrm{~s}, 3 \mathrm{H}), 3.05-2.96(\mathrm{~m}, 4 \mathrm{H}), 2.70(\mathrm{~m}, 1 \mathrm{H}), 2.44-2.38(\mathrm{~m}, 1 \mathrm{H}), 2.17-2.12(\mathrm{~m}, 2 \mathrm{H}) .{ }^{13} \mathrm{C}$ $\operatorname{NMR}\left(126 \mathrm{MHz}, \mathrm{CDCl}_{3}\right) \delta 197.8,163.7,161.9(J=243.2 \mathrm{~Hz}), 147.7,146.0,139.7,130.8,125.6$, 125.5, 114.3, 113.6, 112.5, 110.7, 58.8, 55.5, 35.6, 34.0, 30.0, 26.5. ${ }^{19} \mathrm{~F}$ NMR $\left(376 \mathrm{MHz}, \mathrm{CDCl}_{3}\right) \delta$ -117.15. HRMS (ESI) calculated for [M+H, $\left.\mathrm{C}_{19} \mathrm{H}_{18} \mathrm{FO}_{2}\right]^{+}$: 297.1285; found: 297.1285.
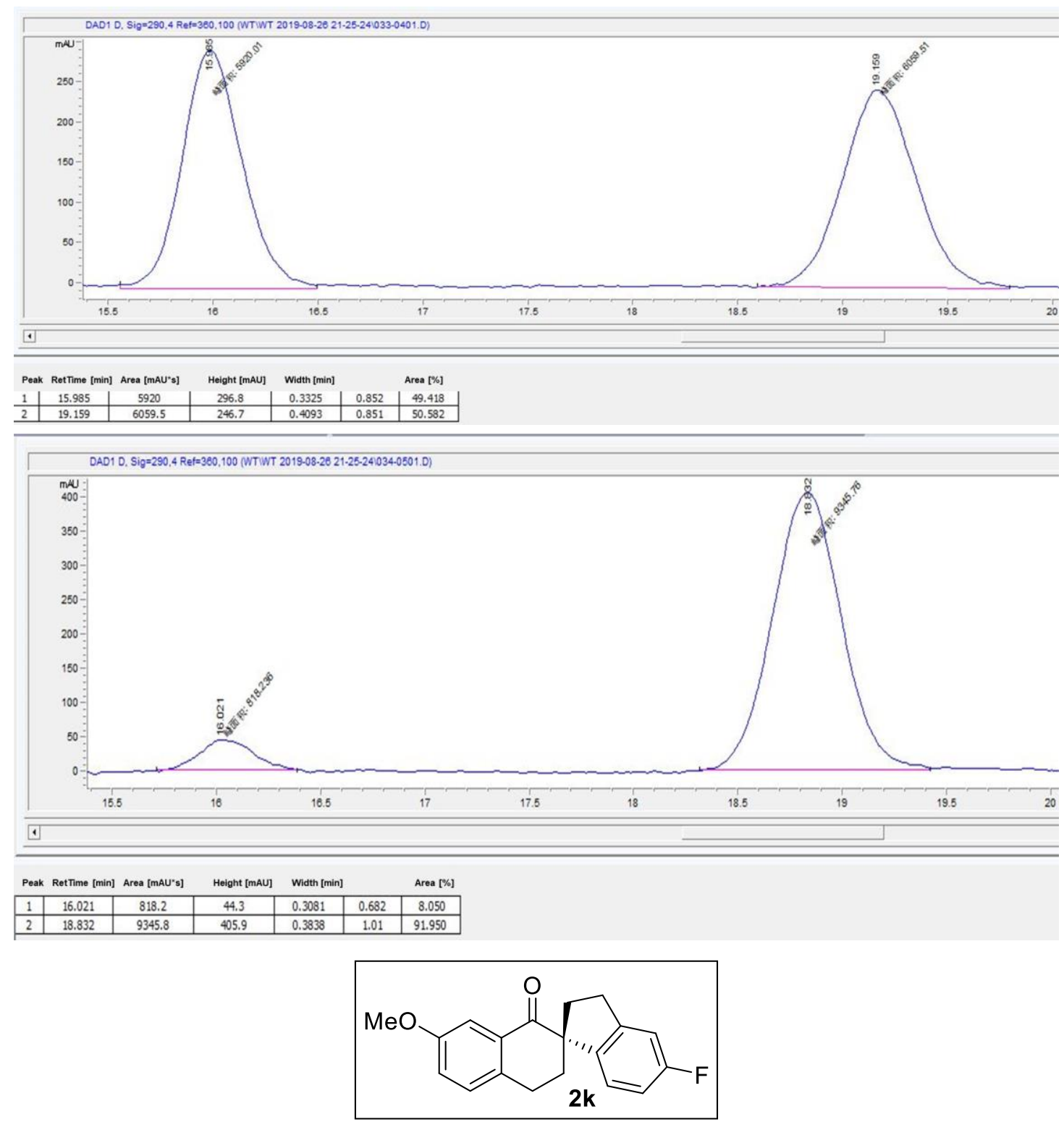


\section{(S)-5-Fluoro-7'-methoxy-2,3,3',4'-tetrahydro-1'H-spiro[indene-1,2'-naphthalen]-1'-one (2k):}

White solid. $94 \%$ yield, $90 \%$ ee. ( silica gel, eluent, petroleum ether/ethyl acetate $=20: 1$ ). Chiral HPLC conditions: chiralcel IC column, $\mathrm{n}$-hexane/ isopropanol = 95: 5, flow rate $1.0 \mathrm{~mL} / \mathrm{min}, 290$ $\mathrm{nm}, t_{1}=11.85 \mathrm{~min}$ (major), $t_{2}=13.15 \mathrm{~min}$ (minor). $[\alpha]_{\mathrm{D}}{ }^{25}=+23.8\left(\mathrm{c}=1.18, \mathrm{CHCl}_{3}\right) .{ }^{1} \mathrm{H} \mathrm{NMR}(500$ $\left.\mathrm{MHz}, \mathrm{CDCl}_{3}\right) \delta 8.09(\mathrm{~d}, J=7.8 \mathrm{~Hz}, 1 \mathrm{H}), 7.52(\mathrm{t}, J=7.4 \mathrm{~Hz}, 1 \mathrm{H}), 7.35(\mathrm{t}, J=7.5 \mathrm{~Hz}, 1 \mathrm{H}), 7.30(\mathrm{~d}$, $J=7.7 \mathrm{~Hz}, 1 \mathrm{H}), 6.97(\mathrm{dd}, J=8.9 \mathrm{~Hz}, J=1.2 \mathrm{~Hz}, 1 \mathrm{H}), 6.91(\mathrm{dd}, J=8.3 \mathrm{~Hz}, J=5.2 \mathrm{~Hz}, 1 \mathrm{H}), 6.82$ $(\mathrm{td}, J=8.5 \mathrm{~Hz}, J=1.9 \mathrm{~Hz}, 1 \mathrm{H}), 3.16-3.05(\mathrm{~m}, 2 \mathrm{H}), 3.05-2.95(\mathrm{~m}, 2 \mathrm{H}), 2.77-2.62(\mathrm{~m}, 1 \mathrm{H}), 2.45$ (m, 1H), $2.22-2.07(\mathrm{~m}, 2 \mathrm{H}) .{ }^{13} \mathrm{C} \mathrm{NMR}\left(126 \mathrm{MHz}, \mathrm{CDCl}_{3}\right) \delta 199.3,162.7(\mathrm{~J}=239.4 \mathrm{~Hz}), 158.5$, 146.6, 141.0, 136.2, 132.7, 130.0, 124.6, 121.9, 113.3, 111.9, 110.1, 58.0, 55.5, 35.3, 34.3, 30.2, 25.4. ${ }^{19} \mathrm{~F}$ NMR (376 MHz, $\left.\mathrm{CDCl}_{3}\right) \delta-115.88$ (s). HRMS (ESI) calculated for $\left[\mathrm{M}+\mathrm{H}, \mathrm{C}_{19} \mathrm{H}_{18} \mathrm{FO}_{2}\right]^{+}$: 297.1285; found: 297.1286 .
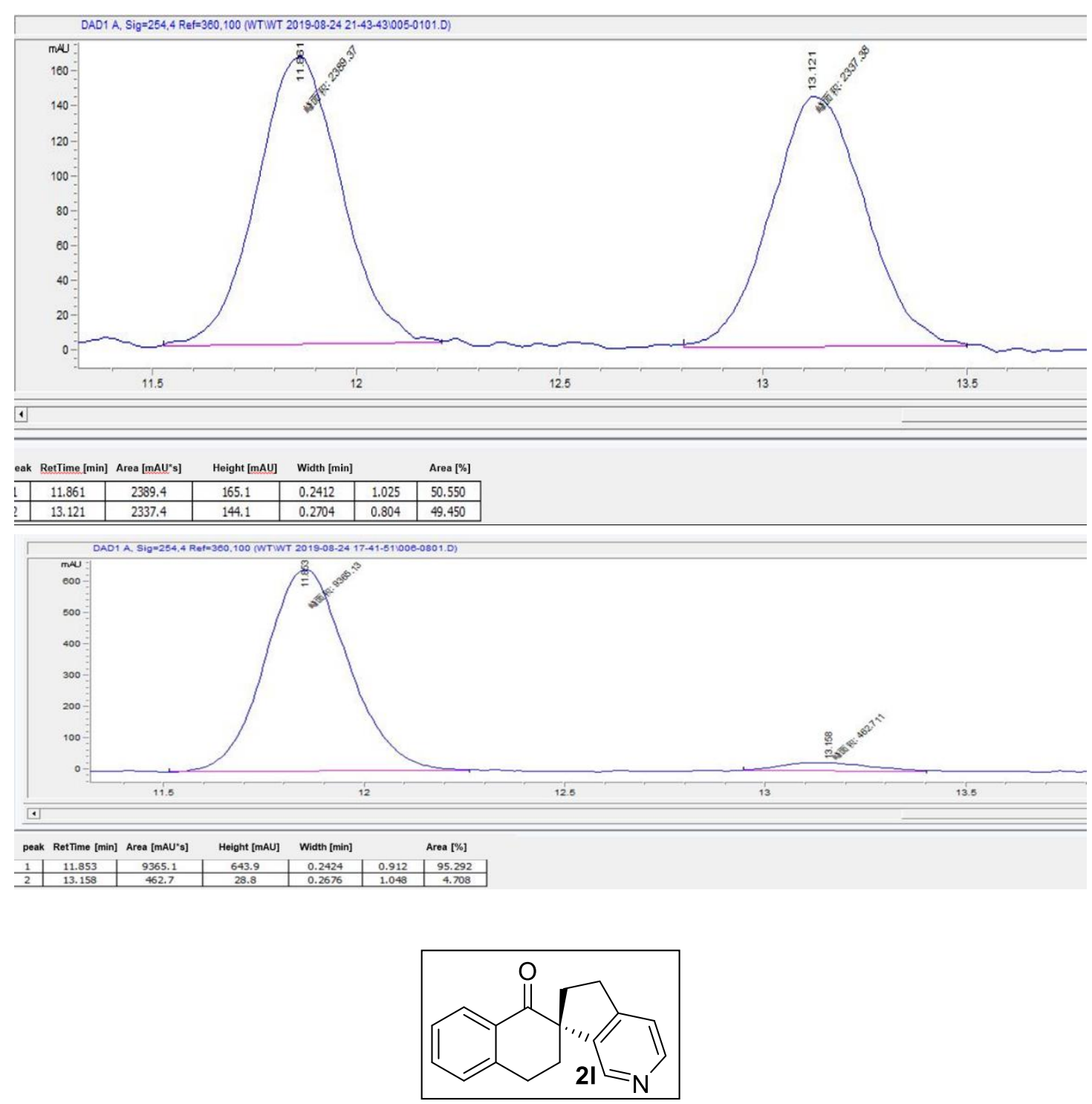
(S)-3',4',5,6-Tetrahydro-1'H-spiro[cyclopenta[c]pyridine-7,2'-naphthalen]-1'-one(2I): Brown solid. $68 \%$ yield, $80 \%$ ee. (silica gel, eluent, petroleum ether/ethyl acetate $=20: 1$ ). Chiral HPLC conditions: chiralcel IC column, $\mathrm{n}$-hexane/ isopropanol $=60: 40$, flow rate $1.0 \mathrm{~mL} / \mathrm{min}, 254 \mathrm{~nm}, t_{1}$ $=13.78 \mathrm{~min}$ (major), $t_{2}=21.0 \mathrm{~min}$ (minor). $[\alpha]_{\mathrm{D}}{ }^{25}=+55.7\left(\mathrm{c}=0.64, \mathrm{CHCl}_{3}\right) .{ }^{1} \mathrm{H} \mathrm{NMR}(500 \mathrm{MHz}$, $\left.\mathrm{CDCl}_{3}\right) \delta 8.45(\mathrm{~d}, J=5.0 \mathrm{~Hz}, 1 \mathrm{H}), 8.26(\mathrm{~s}, 1 \mathrm{H}), 8.07(\mathrm{dd}, J=7.9 \mathrm{~Hz}, J=1.0 \mathrm{~Hz}, 1 \mathrm{H}), 7.54(\mathrm{td}, J=$ $7.5 \mathrm{~Hz}, J=1.4 \mathrm{~Hz}, 1 \mathrm{H}), 7.36(\mathrm{t}, J=7.5 \mathrm{~Hz}, 1 \mathrm{H}), 7.31(\mathrm{~d}, J=7.7 \mathrm{~Hz}, 1 \mathrm{H}), 7.26(\mathrm{~d}, J=3.1 \mathrm{~Hz}, 1 \mathrm{H})$, 3.16 - $2.99(\mathrm{~m}, 4 \mathrm{H}), 2.69(\mathrm{~m}, 1 \mathrm{H}), 2.56-2.50(\mathrm{~m}, 1 \mathrm{H}), 2.23(\mathrm{~m}, 1 \mathrm{H}), 2.15(\mathrm{~m}, 1 \mathrm{H}) .{ }^{13} \mathrm{C}$ NMR $(126$ $\left.\mathrm{MHz}, \mathrm{CDCl}_{3}\right) \delta 198.3,154.4,148.3,145.2,143.4,141.9,133.7,131.6,128.9,128.3,127.0,120.5$, 57.9, 34.7, 33.9, 30.3, 26.0. HRMS (ESI) calculated for $\left[\mathrm{M}+\mathrm{H}, \mathrm{C}_{17} \mathrm{H}_{16} \mathrm{NO}\right]^{+}: 250.1226$; found: 250.1229 .

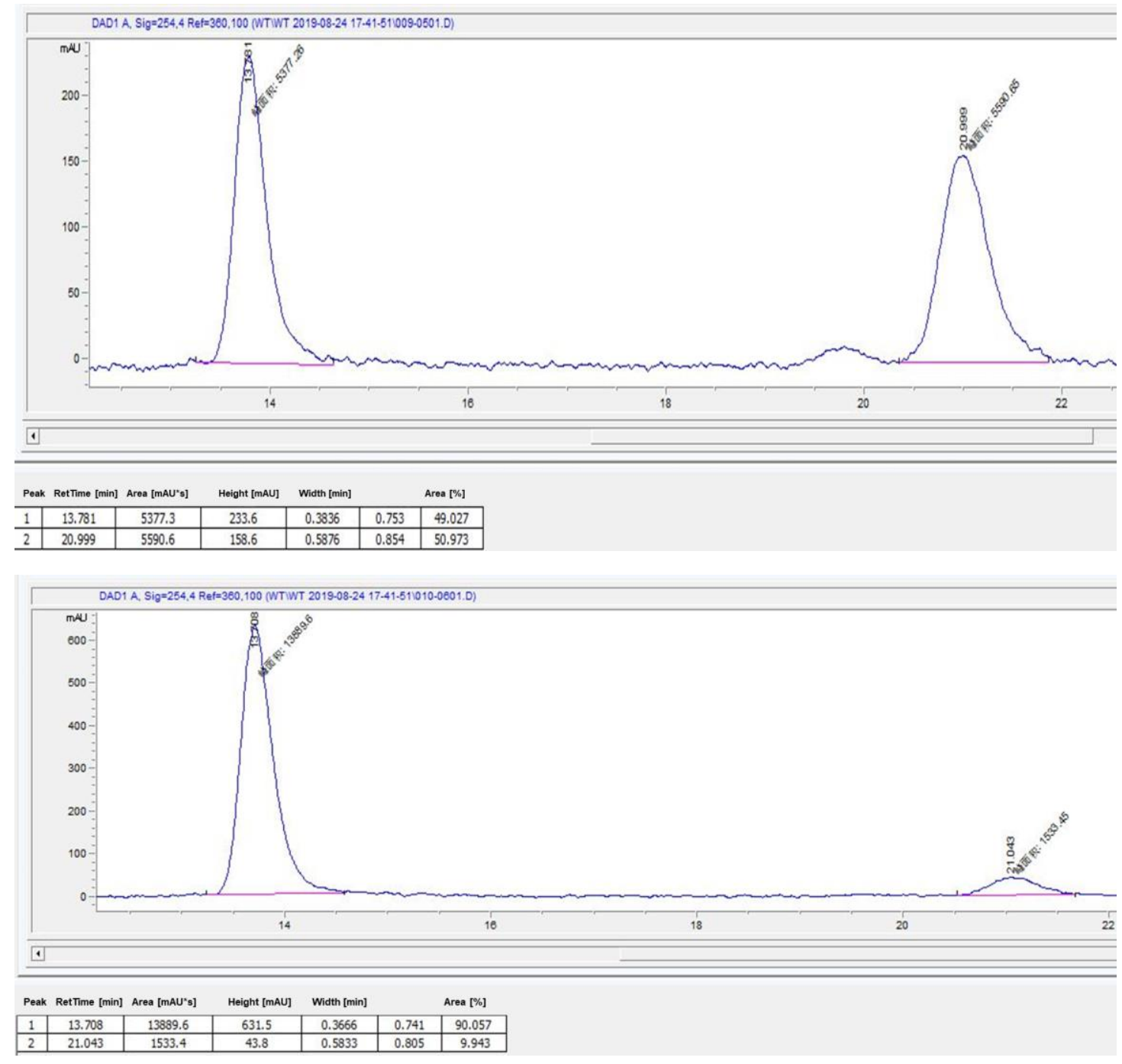




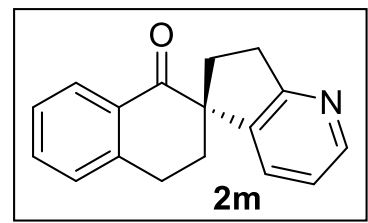

(R)-3',4',6,7-Tetrahydro-1'H-spiro[cyclopenta[b]pyridine-5,2'-naphthalen]-1'-one(2m):

Yellow solid. 79\% yield, $82 \%$ ee. (silica gel, eluent, petroleum ether/ethyl acetate $=10: 1$ ). Chiral HPLC conditions: chiralcel IC column, $n$-hexane/ isopropanol = 70: 30, flow rate $1.0 \mathrm{~mL} / \mathrm{min}, 290$ $\mathrm{nm}, t_{1}=17.90 \mathrm{~min}$ (minor), $t_{2}=20.98 \min$ (major). $[\alpha]_{\mathrm{D}}{ }^{25}=+25.2\left(\mathrm{c}=1.05, \mathrm{CHCl}_{3}\right) .{ }^{1} \mathrm{H}$ NMR $(500$ $\left.\mathrm{MHz}, \mathrm{CDCl}_{3}\right) \delta 8.43(\mathrm{~d}, J=4.7 \mathrm{~Hz}, 1 \mathrm{H}), 8.08(\mathrm{~d}, J=7.8 \mathrm{~Hz}, 1 \mathrm{H}), 7.53(\mathrm{t}, J=7.5 \mathrm{~Hz}, 1 \mathrm{H}), 7.36(\mathrm{t}$, $J=7.5 \mathrm{~Hz}, 1 \mathrm{H}), 7.30(\mathrm{dd}, J=11.5, \mathrm{~Hz}, J=7.7 \mathrm{~Hz}, 2 \mathrm{H}), 7.06(\mathrm{dd}, J=7.4, \mathrm{~Hz}, J=5.1 \mathrm{~Hz}, 1 \mathrm{H}), 3.20$ - $3.12(\mathrm{~m}, 3 \mathrm{H}), 3.04(\mathrm{~m}, 1 \mathrm{H}), 2.67(\mathrm{~m}, 1 \mathrm{H}), 2.45(\mathrm{~m}, 1 \mathrm{H}), 2.24-2.14(\mathrm{~m}, 2 \mathrm{H}) .{ }^{13} \mathrm{C} \mathrm{NMR}(126 \mathrm{MHz}$, $\left.\mathrm{CDCl}_{3}\right) \delta$ 198.7, 165.1, 148.6, 143.4, 138.9, 133.7, 131.8, 131.7, 128.9, 128.3, 127.0, 121.3, 57.2, 33.9, 32.4, 31.9, 25.8. HRMS (ESI) calculated for [M+H, $\left.\mathrm{C}_{17} \mathrm{H}_{16} \mathrm{NO}\right]^{+}: 250.1226$; found: 250.1230 . 

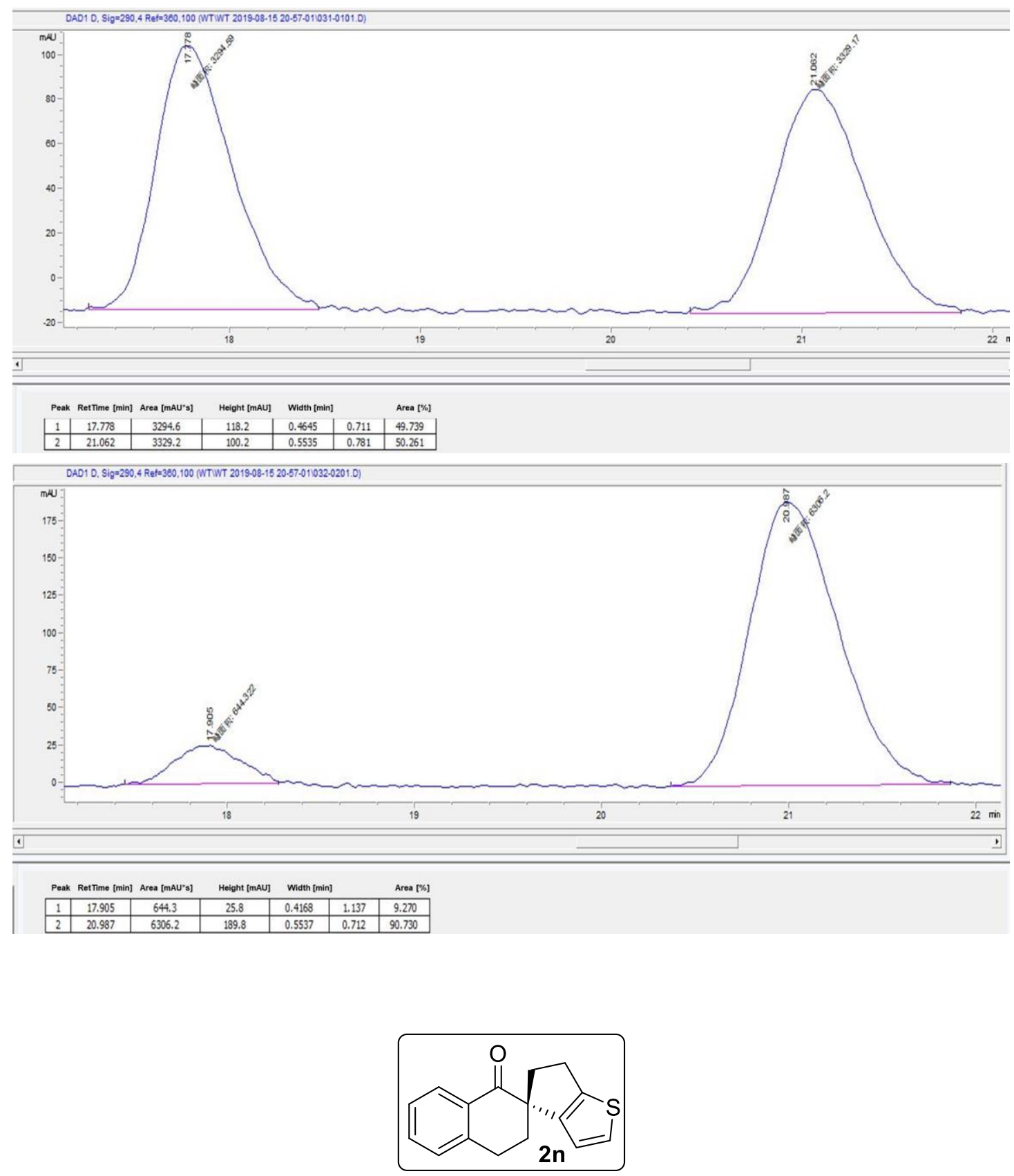

(R)-3',4',5,6-tetrahydro-1'H-spiro[cyclopenta[b]thiophene-4,2'-naphthalen]-1'-one(2n):

White solid. 98\% yield, 58\% ee. ( silica gel, eluent, petroleum ether/ethyl acetate $=20: 1$ ). Chiral HPLC conditions: chiralcel IC column, $\mathrm{n}$-hexane/ isopropanol $=97: 3$, flow rate $1.0 \mathrm{~mL} / \mathrm{min}, 254 \mathrm{~nm}$, $t_{1}=9.44 \min$ (minor), $t_{2}=9.82 \mathrm{~min}$ (major). $[\alpha]_{\mathrm{D}}{ }^{25}=+95.3\left(\mathrm{c}=1.23, \mathrm{CHCl}_{3}\right) .{ }^{1} \mathrm{H} \mathrm{NMR}(500 \mathrm{MHz}$, $\left.\mathrm{CDCl}_{3}\right) \delta 8.06(\mathrm{~d}, J=7.8 \mathrm{~Hz}, 1 \mathrm{H}), 7.51(\mathrm{t}, J=7.5 \mathrm{~Hz}, 1 \mathrm{H}), 7.36-7.28(\mathrm{~m}, 2 \mathrm{H}), 7.09(\mathrm{~d}, J=5.0 \mathrm{~Hz}$, 1H), $6.52(\mathrm{~d}, J=5.0 \mathrm{~Hz}, 1 \mathrm{H}), 3.29-3.18(\mathrm{~m}, 2 \mathrm{H}), 3.12-3.00(\mathrm{~m}, 3 \mathrm{H}), 2.39-2.31(\mathrm{~m}, 2 \mathrm{H}), 2.30$ $-2.23(\mathrm{~m}, 1 \mathrm{H}) .{ }^{13} \mathrm{C}$ NMR $\left(126 \mathrm{MHz}, \mathrm{CDCl}_{3}\right) \delta 198.7,146.8,143.7,143.6,133.3,131.9,128.8$, 128.3, 128.0, 126.8, 121.2, 56.2, 39.5, 34.7, 27.3, 26.5. HRMS (ESI) calculated for $[\mathrm{M}+\mathrm{H}$, $\left.\mathrm{C}_{16} \mathrm{H}_{15} \mathrm{OS}\right]^{+}:$255.0838; found: 255.0840 . 

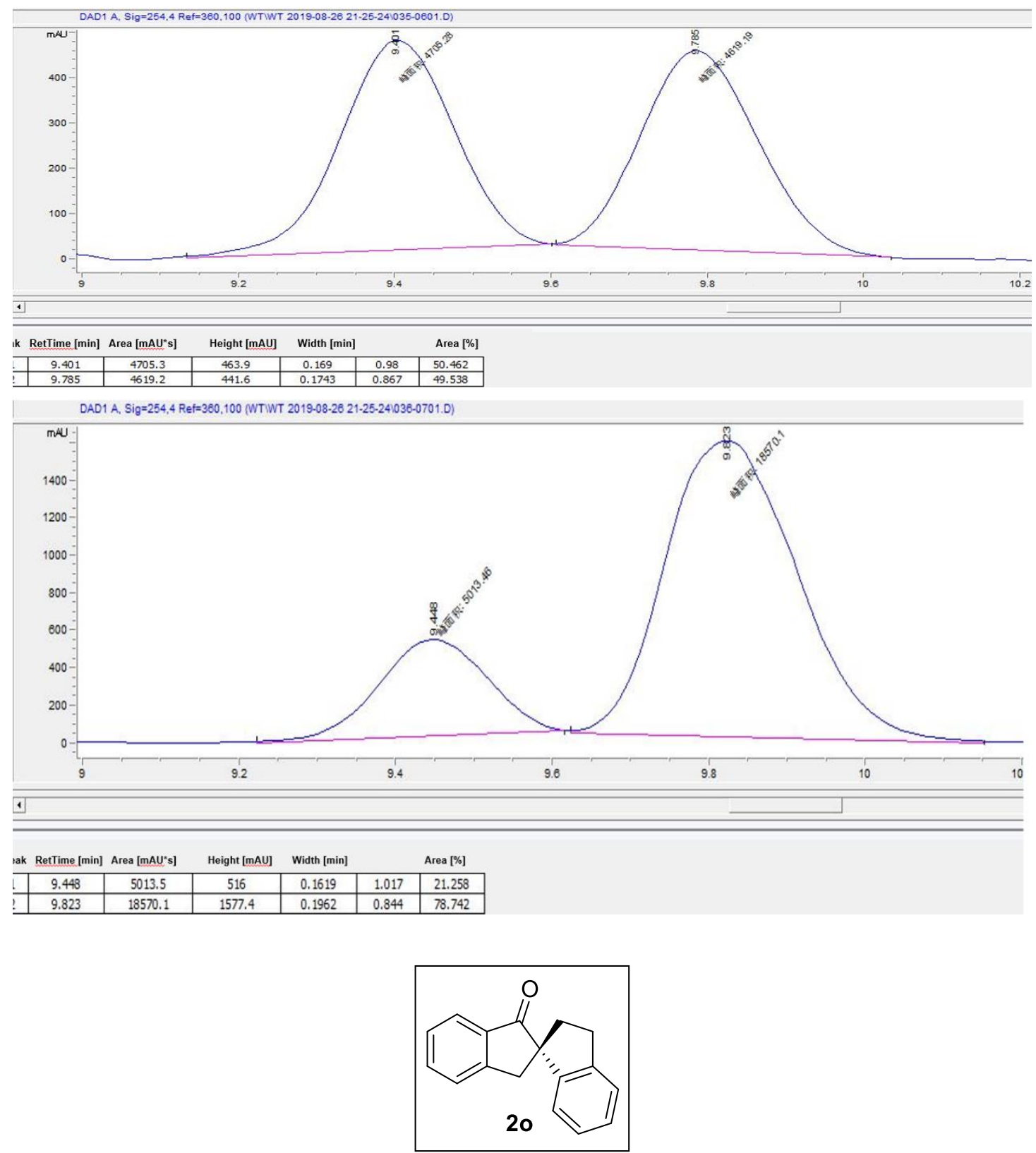

(S)-2,3-Dihydro-1,2'-spirobi[inden]-1'(3'H)-one(2o): Yellow oil. 98\% yield, 84\% ee. (silica gel, eluent, petroleum ether/ethyl acetate $=20: 1$ ). Chiral HPLC conditions: chiralcel IC column, nhexane/ isopropanol $=95: 5$, flow rate $1.0 \mathrm{~mL} / \mathrm{min}, 290 \mathrm{~nm}, t_{1}=11.86 \mathrm{~min}$ (minor), $t_{2}=15.84 \mathrm{~min}$ (major). $[\alpha]_{\mathrm{D}}{ }^{25}=+108.5\left(\mathrm{c}=1.15, \mathrm{CHCl}_{3}\right) .{ }^{1} \mathrm{H} \mathrm{NMR}\left(500 \mathrm{MHz}, \mathrm{CDCl}_{3}\right) \delta 7.80(\mathrm{~d}, J=7.6 \mathrm{~Hz}, 1 \mathrm{H})$, $7.67-7.63(\mathrm{~m}, 1 \mathrm{H}), 7.53-7.50(\mathrm{~m}, 1 \mathrm{H}), 7.43$ (t, $J=7.5 \mathrm{~Hz}, 1 \mathrm{H}), 7.30$ (d, $J=7.6 \mathrm{~Hz}, 1 \mathrm{H}), 7.19$ (dd, $J=10.8 \mathrm{~Hz}, \mathrm{~J}=4.1 \mathrm{~Hz}, 1 \mathrm{H}), 7.08(\mathrm{t}, J=7.5 \mathrm{~Hz}, 1 \mathrm{H}), 6.78(\mathrm{~d}, J=7.6 \mathrm{~Hz}, 1 \mathrm{H}), 3.47-3.37(\mathrm{~m}, 2 \mathrm{H})$, $3.33(\mathrm{~m}, 1 \mathrm{H}), 3.11-3.03(\mathrm{~m}, 1 \mathrm{H}), 2.65(\mathrm{~m}, 1 \mathrm{H}), 2.18(\mathrm{~m}, 1 \mathrm{H}) .{ }^{13} \mathrm{C} \mathrm{NMR}\left(126 \mathrm{MHz}, \mathrm{CDCl}_{3}\right) \delta 208.3$, 153.0, 146.2, 144.6, 135.8, 134.9, 127.7, 127.4, 126.7, 126.3, 124.8, 124.7, 122.1, 61.9, 42.7, 37.6, 31.6. HRMS (ESI) calculated for $\left[\mathrm{M}+\mathrm{H}, \mathrm{C}_{17} \mathrm{H}_{15} \mathrm{O}\right]^{+}: 235.1117$; found: 235.1120 . 

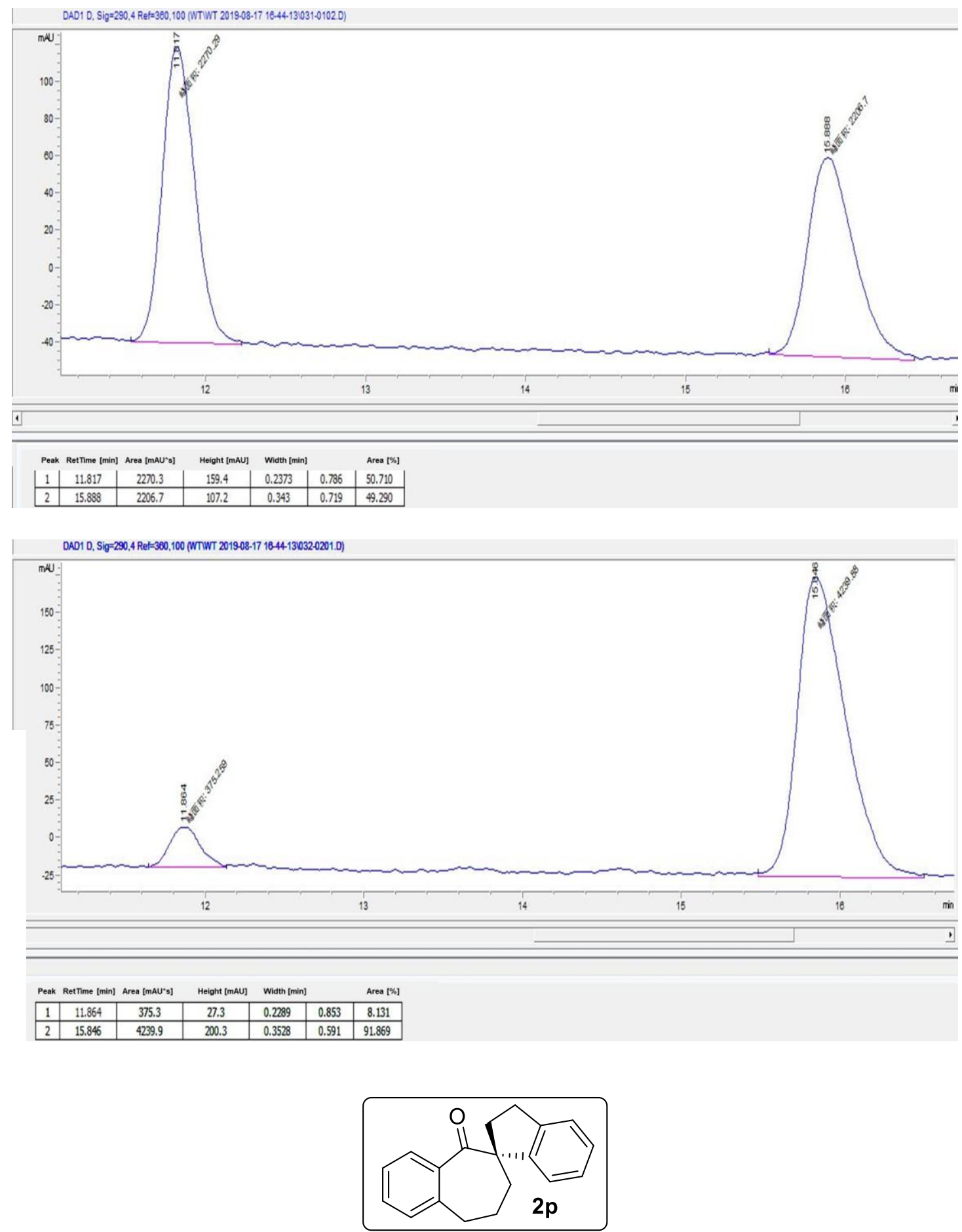

(R)-2',3',8,9-Tetrahydrospiro[benzo[7] annulene-6,1'-inden]-5(7H)-one(2p): Yellow oil. 98\% yield, $90 \%$ ee. ( silica gel, eluent, petroleum ether/ethyl acetate $=20: 1$ ). Chiral HPLC conditions: chiralcel IC column, $\mathrm{n}$-hexane/ isopropanol $=95: 5$, flow rate $1.0 \mathrm{~mL} / \mathrm{min}, 290 \mathrm{~nm}, t_{1}=11.08 \mathrm{~min}$ (major), $t_{2}=11.63 \min$ (minor). $[\alpha]_{\mathrm{D}}^{25}=+15.3\left(\mathrm{c}=0.96, \mathrm{CHCl}_{3}\right) .{ }^{1} \mathrm{H}$ NMR $\left(500 \mathrm{MHz}, \mathrm{CDCl}_{3}\right) \delta$ 
$7.40(\mathrm{td}, J=7.3 \mathrm{~Hz}, J=1.9 \mathrm{~Hz}, 1 \mathrm{H}), 7.32-7.27(\mathrm{~m}, 2 \mathrm{H}), 7.25(\mathrm{~s}, 1 \mathrm{H}), 7.22-7.15(\mathrm{~m}, 2 \mathrm{H}), 7.13(\mathrm{t}$, $J=7.4 \mathrm{~Hz}, 1 \mathrm{H}), 6.98(\mathrm{~d}, J=7.6 \mathrm{~Hz}, 1 \mathrm{H}), 3.20-3.12(\mathrm{~m}, 1 \mathrm{H}), 3.02-2.85(\mathrm{~m}, 3 \mathrm{H}), 2.66-2.57(\mathrm{~m}$, 1H), $2.25-2.18(\mathrm{~m}, 1 \mathrm{H}), 2.14(\mathrm{~m}, 1 \mathrm{H}), 2.08-2.00(\mathrm{~m}, 1 \mathrm{H}), 1.94(\mathrm{~m}, 1 \mathrm{H}), 1.84(\mathrm{dt}, J=14.2,4.6 \mathrm{~Hz}$, 1H). ${ }^{13} \mathrm{C}$ NMR (126 MHz, $\left.\mathrm{cdcl}_{3}\right) \delta 213.0,147.8,143.4,141.5,137.4,131.0,128.5,127.4,127.0$, $126.8,126.4,124.8,123.7,62.8,35.4,34.8,32.7,31.1,23.2$. HRMS (ESI) calculated for $[\mathrm{M}+\mathrm{H}$, $\left.\mathrm{C}_{19} \mathrm{H}_{19} \mathrm{O}\right]^{+}: 263.1430$; found: 263.1432 .
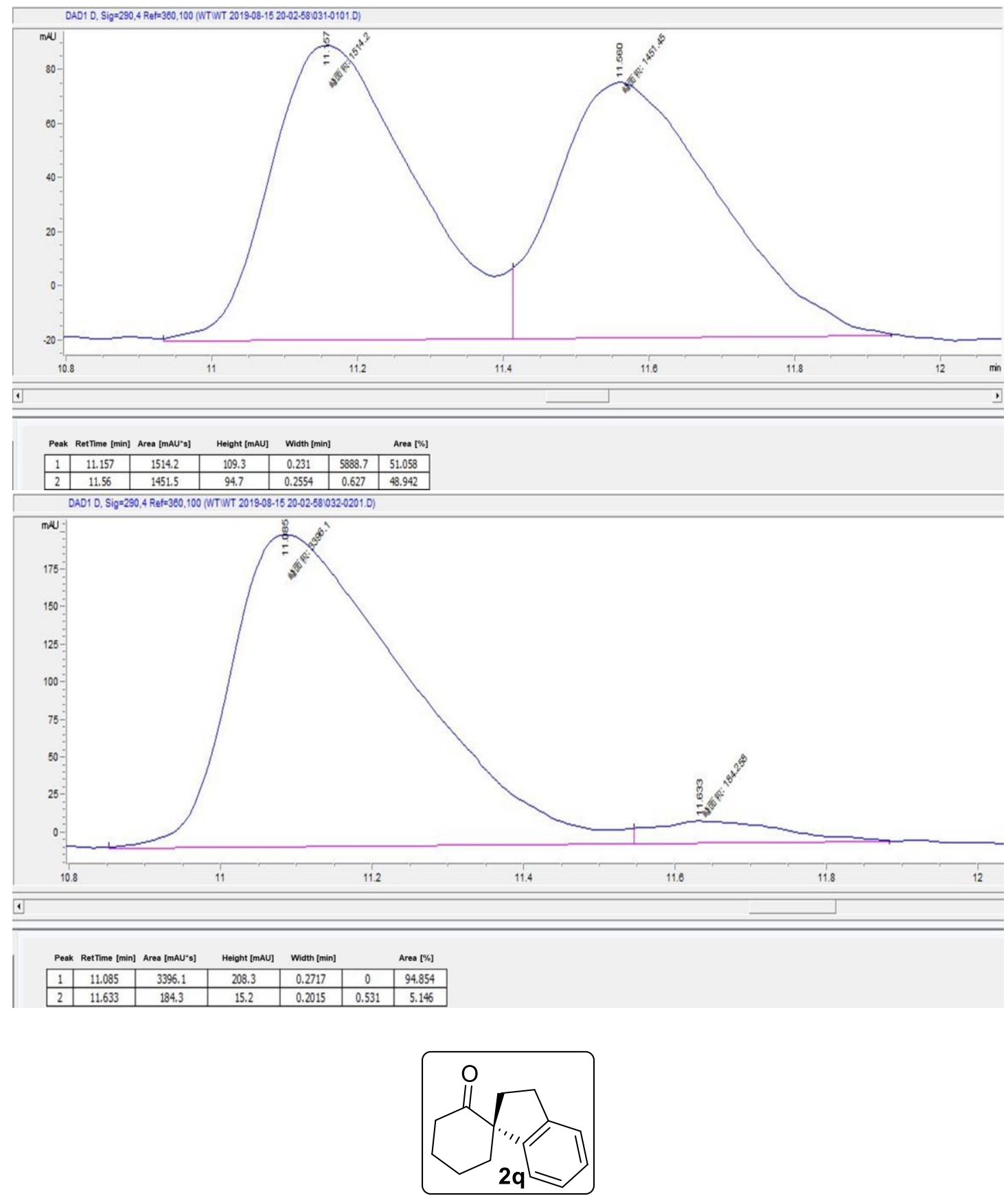
(R)-2',3'-Dihydrospiro[cyclohexane-1,1'-inden]-2-one(2q): Yellow oil. 75\% yield, $85 \%$ ee. ( silica gel, eluent, petroleum ether/ethyl acetate $=5: 1$ ). Chiral HPLC conditions: chiralcel IC column, n-hexane/ isopropanol = 88: 2 , flow rate $1.0 \mathrm{~mL} / \mathrm{min}, 290 \mathrm{~nm}, t_{1}=8.34 \mathrm{~min}$ (major), $t_{2}=9.06 \mathrm{~min}$ (minor). $[\alpha]_{\mathrm{D}}{ }^{25}=-64.9\left(\mathrm{c}=1.03, \mathrm{CHCl}_{3}\right) .{ }^{1} \mathrm{H} \mathrm{NMR}\left(500 \mathrm{MHz}, \mathrm{CDCl}_{3}\right) \delta 7.25(\mathrm{~d}, J=4.2 \mathrm{~Hz}, 2 \mathrm{H})$, $7.22(\mathrm{t}, J=3.9 \mathrm{~Hz}, 2 \mathrm{H}), 2.92(\mathrm{t}, J=7.2 \mathrm{~Hz}, 2 \mathrm{H}), 2.60-2.53(\mathrm{~m}, 2 \mathrm{H}), 2.38(\mathrm{~m}, 1 \mathrm{H}), 2.16(\mathrm{~m}, 1 \mathrm{H})$, 2.11 - 2.02 (m, 2H), 1.99 - 1.82 (m, 4H). ${ }^{13} \mathrm{C}$ NMR (126 MHz, $\left.\mathrm{CDCl}_{3}\right)$ \& 212.2, 145.1, 143.7, 127.4, 126.3, 124.7, 124.6, 62.6, 39.7, 39.1, 36.5, 30.3, 27.1, 22.5. HRMS (ESI) calculated for $[\mathrm{M}+\mathrm{H}$, $\left.\mathrm{C}_{14} \mathrm{H}_{17} \mathrm{O}\right]^{+}:$201.1274; found: 201.1278.
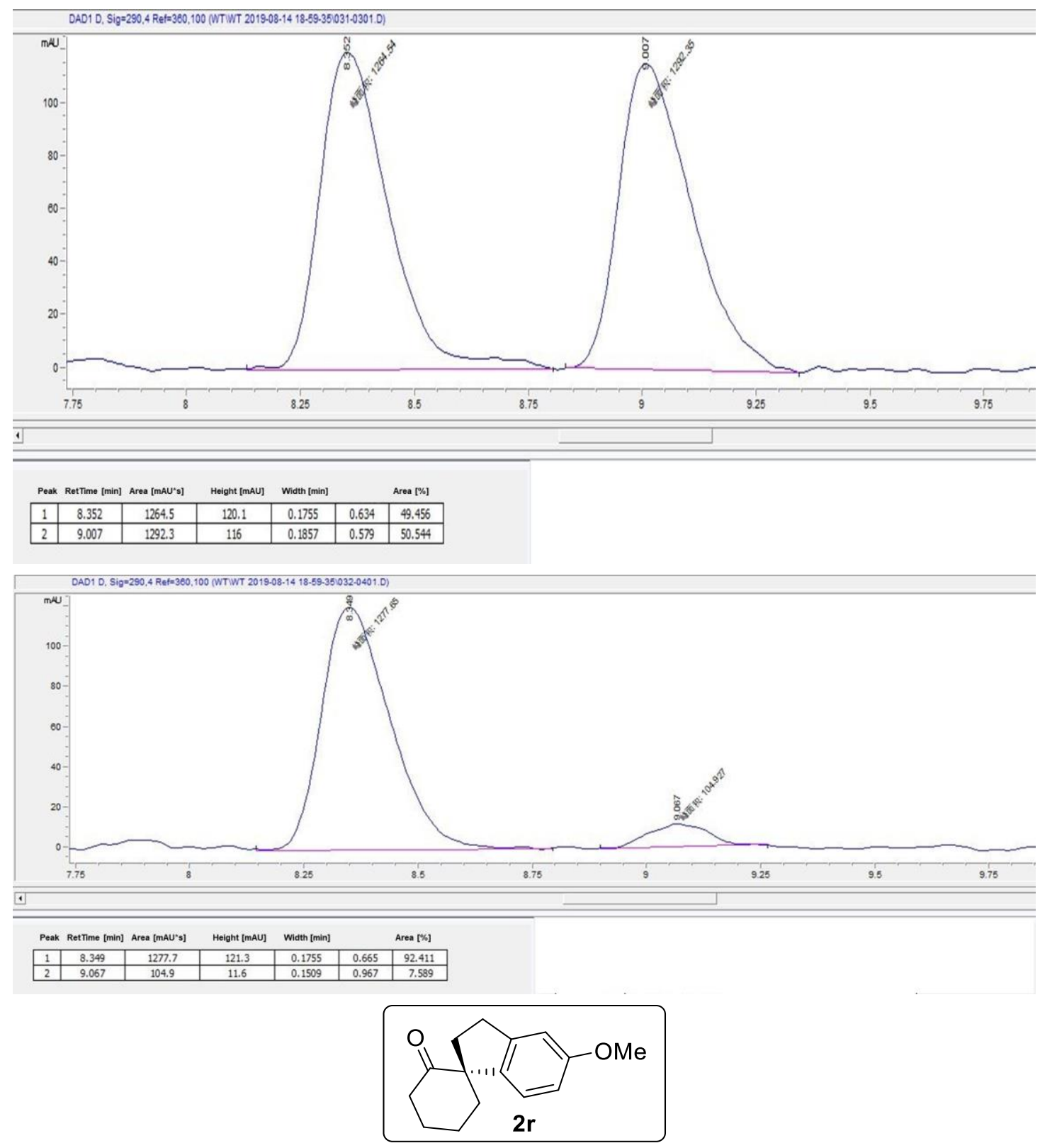

(R)-5'-Methoxy-2',3'-dihydrospiro[cyclohexane-1,1'-inden]-2-one(2r): Yellow oil. $63 \%$ yield, $89 \%$ ee. (silica gel, eluent, petroleum ether/ethyl acetate $=5: 1$ ). Chiral HPLC conditions: chiralcel 
IC column, $\mathrm{n}$-hexane/isopropanol $=85: 15$, flow rate $1.0 \mathrm{~mL} / \mathrm{min}, 290 \mathrm{~nm}, t_{1}=13.57 \mathrm{~min}$ (major), $t_{2}=16.44 \min$ (minor). $[\alpha]_{\mathrm{D}}{ }^{25}=-64.3\left(\mathrm{c}=1.0, \mathrm{CHCl}_{3}\right) .{ }^{1} \mathrm{H} \mathrm{NMR}\left(500 \mathrm{MHz}, \mathrm{CDCl}_{3}\right) \delta 7.16(\mathrm{~d}, J=$ $8.4 \mathrm{~Hz}, 1 \mathrm{H}), 6.79-6.75(\mathrm{~m}, 2 \mathrm{H}), 3.78(\mathrm{~s}, 3 \mathrm{H}), 2.88(\mathrm{t}, J=7.3 \mathrm{~Hz}, 2 \mathrm{H}), 2.55(\mathrm{dd}, J=9.8, J=5.6 \mathrm{~Hz}$, 2H), $2.41-2.36(\mathrm{~m}, 1 \mathrm{H}), 2.14(\mathrm{~m}, 1 \mathrm{H}), 2.07-1.98(\mathrm{~m}, 2 \mathrm{H}), 1.96-1.82(\mathrm{~m}, 4 \mathrm{H}) .{ }^{13} \mathrm{C}$ NMR $(126$ $\left.\mathrm{MHz}, \mathrm{CDCl}_{3}\right) \delta 212.6,159.3,145.3,137.3,125.3,112.4,109.8,61.9,55.3,39.6,39.4,36.8,30.5$, 27.2, 22.5. HRMS (ESI) calculated for $\left[\mathrm{M}+\mathrm{H}, \mathrm{C}_{15} \mathrm{H}_{19} \mathrm{O}_{2}\right]^{+}: 231.1380$; found: 231.1381 .
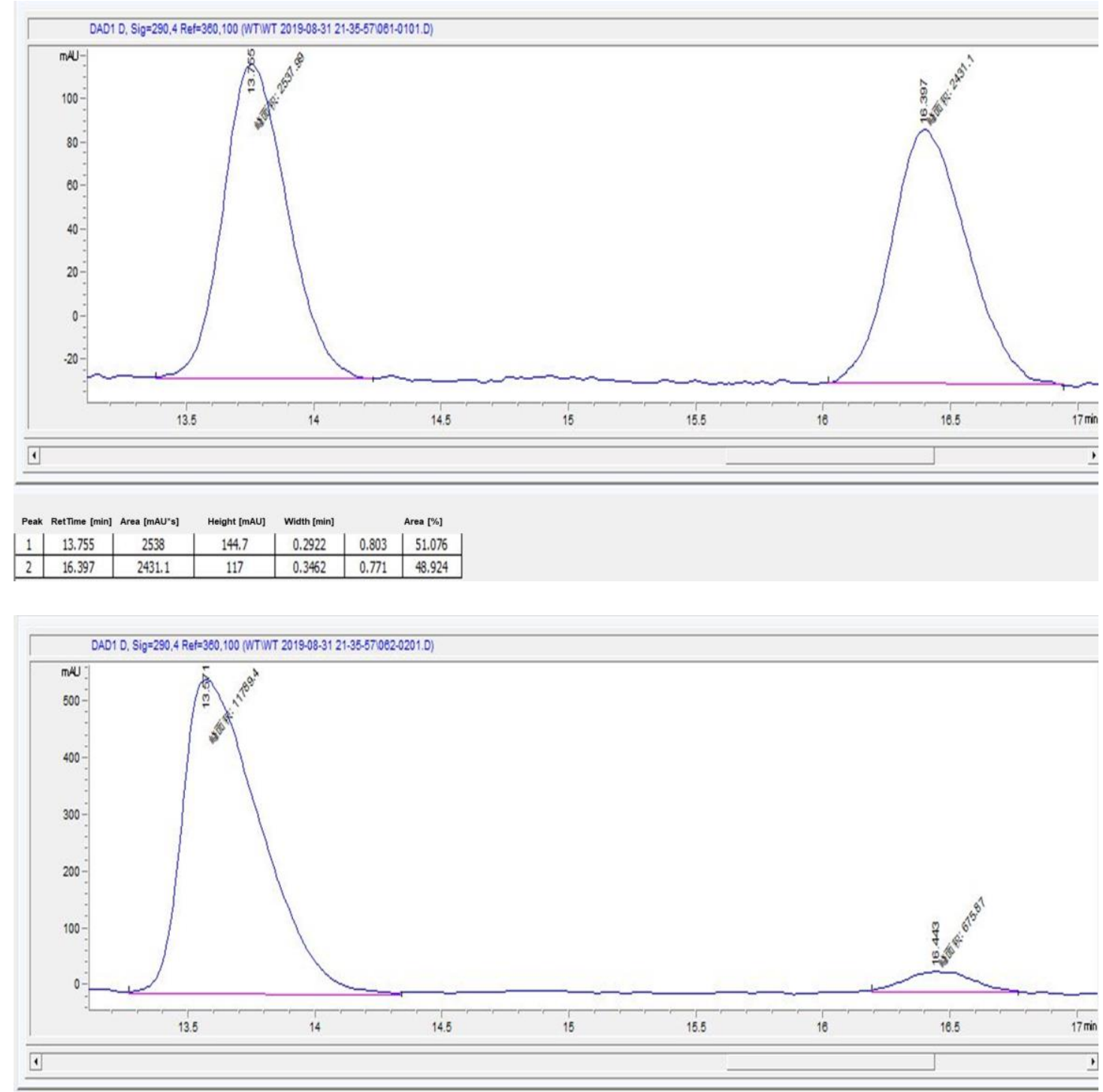

\begin{tabular}{|c|c|c|c|c|c|c|}
\hline \multicolumn{2}{|c|}{ Peak RetTime [min] Area [mAU's] } & Height [mAU] & Width [min] & \multicolumn{2}{c|}{ Area [\%] } \\
\hline 1 & 13.571 & 11799.4 & 552.9 & 0.3554 & 0.446 & 94.578 \\
\hline 2 & 16.443 & 675.9 & 35.9 & 0.3136 & 0.84 & 5.422 \\
\hline
\end{tabular}




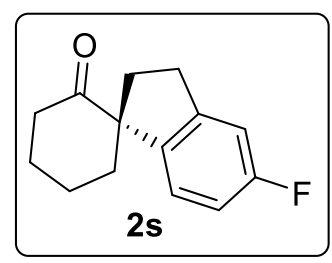

(R)-5'-Fluoro-2',3'-dihydrospiro[cyclohexane-1,1'-inden]-2-one(2s): Yellow oil. 46\% yield, 86\% ee. (silica gel, eluent, petroleum ether/ethyl acetate $=5: 1$ ). Chiral HPLC conditions: chiralcel IC column, $\mathrm{n}$-hexane/ isopropanol $=85: 15$, flow rate $1.0 \mathrm{~mL} / \mathrm{min}, 254 \mathrm{~nm}, t_{1}=6.33 \mathrm{~min}$ (major), $t_{2}=$ $6.80 \mathrm{~min}$ (minor). $[\alpha]_{\mathrm{D}}{ }^{25}=-18.8\left(\mathrm{c}=1.0, \mathrm{CHCl}_{3}\right){ }^{1} \mathrm{H} \mathrm{NMR}\left(500 \mathrm{MHz}, \mathrm{CDCl}_{3}\right) \delta 7.19-7.14(\mathrm{~m}$, $1 \mathrm{H}), 6.91(\mathrm{dd}, J=12.8 \mathrm{~Hz}, J=5.7 \mathrm{~Hz}, 2 \mathrm{H}), 2.89(\mathrm{t}, J=7.3 \mathrm{~Hz}, 2 \mathrm{H}), 2.61-2.50(\mathrm{~m}, 2 \mathrm{H}), 2.41-2.34$ (m, 1H), $2.21(\mathrm{~m}, 1 \mathrm{H}), 2.09$ (dd, $J=9.2 \mathrm{~Hz}, J=3.3 \mathrm{~Hz}, 1 \mathrm{H}), 2.00-1.81(\mathrm{~m}, 5 \mathrm{H}) .{ }^{13} \mathrm{C}$ NMR $(126$ $\left.\mathrm{MHz}, \mathrm{CDCl}_{3}\right) \delta 212.2,162.5(\mathrm{~J}=244.4 \mathrm{~Hz}), 145.8,140.6,125.7,113.4,111.5,61.9,39.6,39.3$, 36.7, 30.3, 27.1, 22.5. ${ }^{19} \mathrm{~F}$ NMR (376 MHz, $\left.\mathrm{CDCl}_{3}\right) \delta-116.21$. HRMS (ESI) calculated for $[\mathrm{M}+\mathrm{H}$, $\left.\mathrm{C}_{14} \mathrm{H}_{17} \mathrm{O}\right]^{+}:$219.1180; found: 219.1183 .
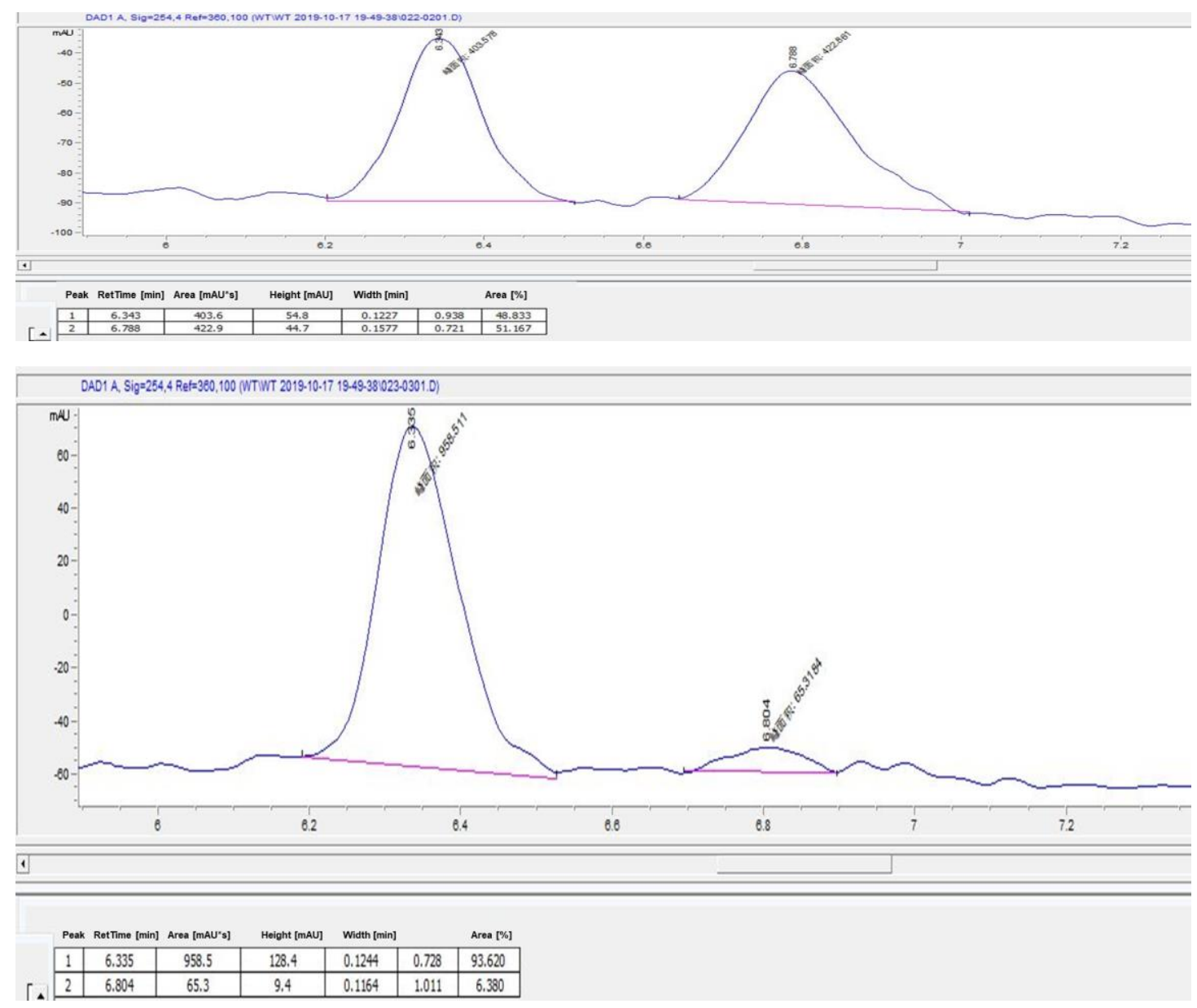


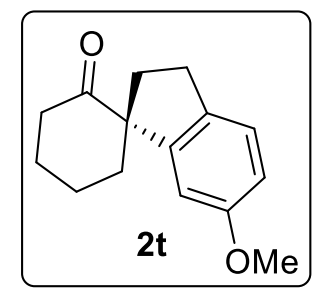

(R)-6'-Methoxy-2',3'-dihydrospiro[cyclohexane-1,1'-inden]-2-one(2t): Yellow oil. 60\% yield, $78 \%$ ee. (silica gel, eluent, petroleum ether/ethyl acetate $=5: 1$ ). Chiral HPLC conditions: chiralcel IC column, $\mathrm{n}$-hexane/ isopropanol $=85: 15$, flow rate $1.0 \mathrm{~mL} / \mathrm{min}, 290 \mathrm{~nm}, t_{1}=10.91 \mathrm{~min}$ (minor), $t_{2}=13.50 \mathrm{~min}$ (major). $[\alpha]_{\mathrm{D}^{25}}=-46.6\left(\mathrm{c}=1.0, \mathrm{CHCl}_{3}\right) .{ }^{1} \mathrm{H} \mathrm{NMR}\left(500 \mathrm{MHz}, \mathrm{CDCl}_{3}\right) \delta 7.11(\mathrm{~d}, J=$ $8.1 \mathrm{~Hz}, 1 \mathrm{H}), 6.76-6.79(\mathrm{~m}, 2 \mathrm{H}), 3.79(\mathrm{~s}, 3 \mathrm{H}), 2.84(\mathrm{t}, J=7.2 \mathrm{~Hz}, 2 \mathrm{H}), 2.56(\mathrm{dt}, J=9.0 \mathrm{~Hz}, J=5.4$ $\mathrm{Hz}, 2 \mathrm{H}), 2.40$ - $2.34(\mathrm{~m}, 1 \mathrm{H}), 2.17$ (m, 1H), 2.08 - 1.99 (m, 2H), $1.94-1.84(\mathrm{~m}, 4 \mathrm{H}) . \mathrm{HRMS}$ (ESI) calculated for $\left[\mathrm{M}+\mathrm{H}, \mathrm{C}_{15} \mathrm{H}_{19} \mathrm{O}_{2}\right]^{+}: 231.1380$; found: 231.1382 .
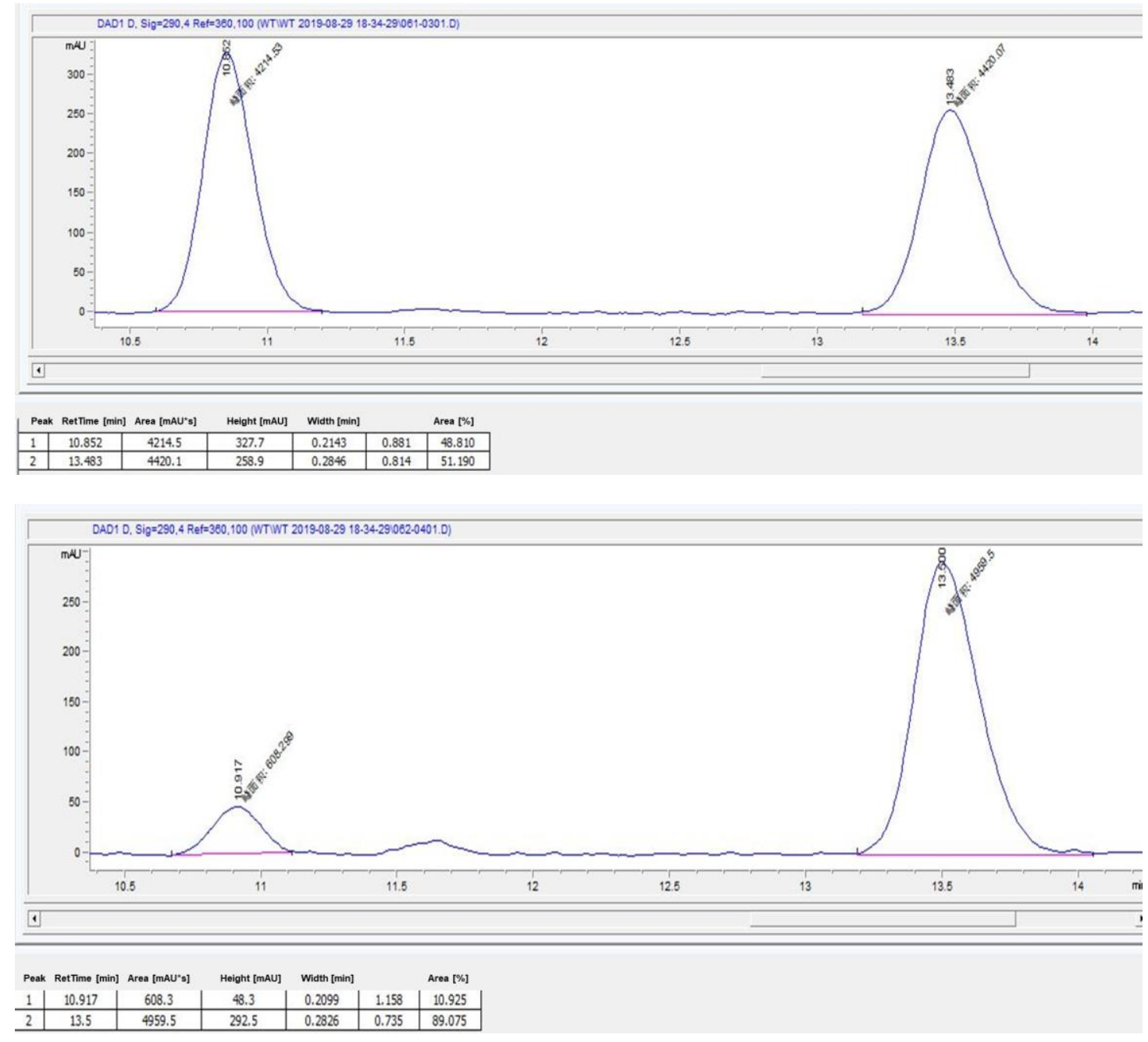


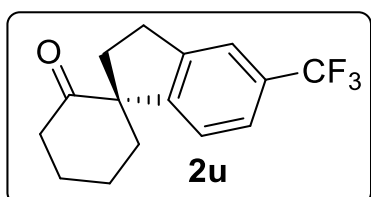

(R)-5'-(Trifluoromethyl)-2',3'-dihydrospiro[cyclohexane-1,1'-inden]-2-one(2u): Yellow oil. 60\% yield, 77\% ee. (silica gel, eluent, petroleum ether/ethyl acetate $=5: 1$ ). Chiral HPLC conditions: chiralcel IC column, $\mathrm{n}$-hexane/ isopropanol $=85: 15$, flow rate $1.0 \mathrm{~mL} / \mathrm{min}, 254 \mathrm{~nm}, t_{1}=5.36 \mathrm{~min}$ (major), $t_{2}=5.90 \mathrm{~min}$ (minor). $[\alpha]_{\mathrm{D}}{ }^{25}=-44.6\left(\mathrm{c}=0.50, \mathrm{CHCl}_{3}\right){ }^{1} \mathrm{H} \mathrm{NMR}\left(500 \mathrm{MHz}, \mathrm{CDCl}_{3}\right) \delta 7.48$ $(\mathrm{d}, J=9.2 \mathrm{~Hz}, 2 \mathrm{H}), 7.32(\mathrm{~d}, J=7.8 \mathrm{~Hz}, 1 \mathrm{H}), 2.96(\mathrm{t}, J=7.3 \mathrm{~Hz}, 2 \mathrm{H}), 2.64-2.57(\mathrm{~m}, 1 \mathrm{H}), 2.55-$ $2.50(\mathrm{~m}, 1 \mathrm{H}), 2.41-2.35(\mathrm{~m}, 1 \mathrm{H}), 2.29-2.24(\mathrm{~m}, 1 \mathrm{H}), 2.15-2.10(\mathrm{~m}, 1 \mathrm{H}), 2.04-1.87(\mathrm{~m}, 5 \mathrm{H}) .{ }^{13} \mathrm{C}$ $\operatorname{NMR}\left(151 \mathrm{MHz}, \mathrm{CDCl}_{3}\right) \delta 210.6,148.1,143.3,128.7(\mathrm{q}, J=273.3 \mathrm{~Hz}), 124.2,122.6,123.4(\mathrm{q}, J=$ $273.3 \mathrm{~Hz}), 120.5,61.6,38.5,37.8,35.2,29.0,26.0,21.2 .{ }^{19} \mathrm{~F}$ NMR $\left(376 \mathrm{MHz}, \mathrm{CDCl}_{3}\right) \delta-62.16$. HRMS (ESI) calculated for [M+Na, $\left.\mathrm{C}_{15} \mathrm{H}_{15} \mathrm{~F}_{3} \mathrm{NaO}\right]^{+}: 291.0967$; found: 291.0968.
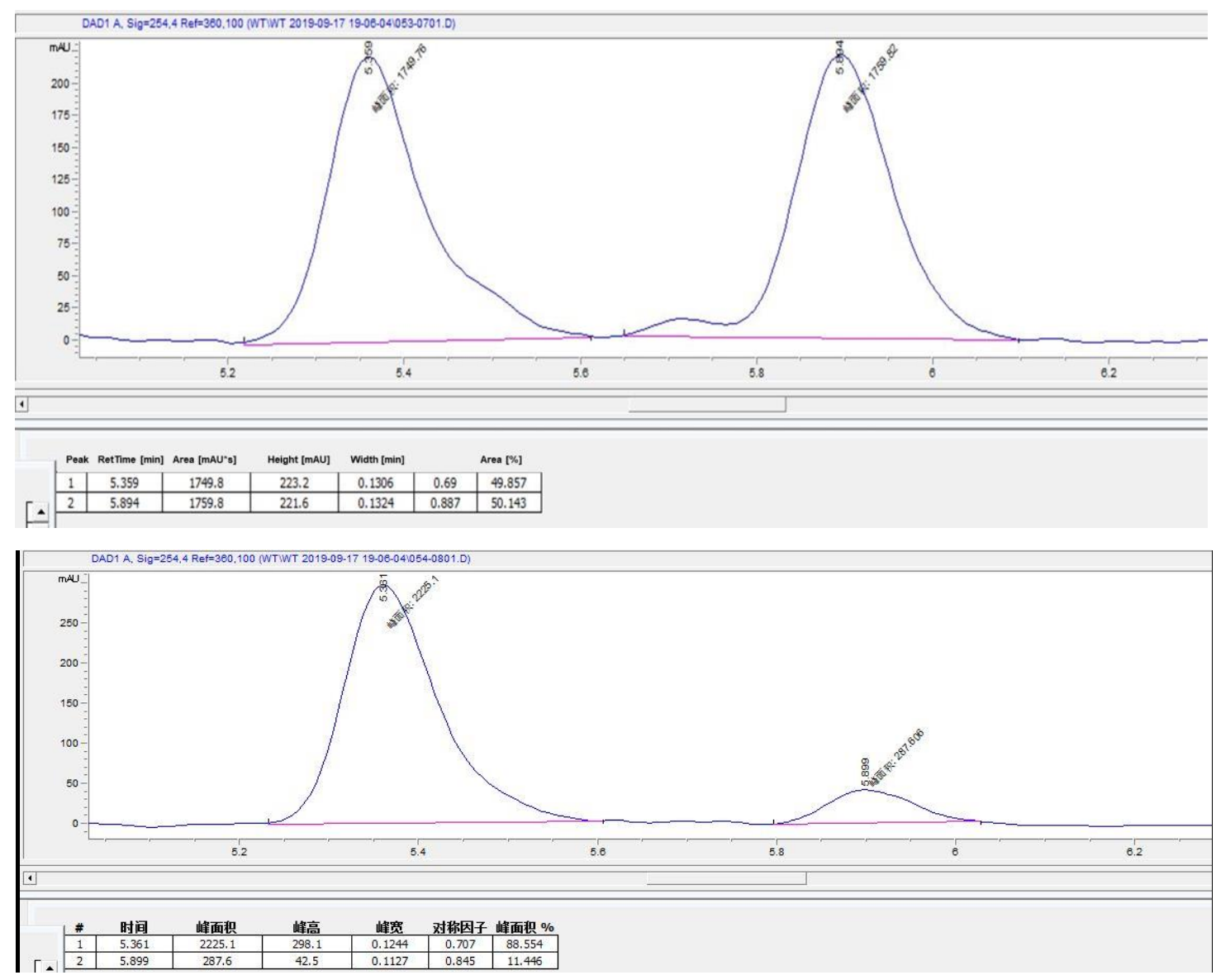


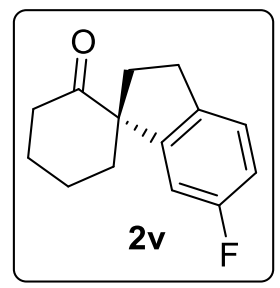

(R)-6'-Fluoro-2',3'-dihydrospiro[cyclohexane-1,1'-inden]-2-one(2v): Yellow oil. 47\% yield, 51\% ee. (silica gel, eluent, petroleum ether/ethyl acetate $=5: 1$ ). Chiral HPLC conditions: chiralcel AD$\mathrm{H}$ column, $\mathrm{n}$-hexane/ isopropanol $=90: 10$, flow rate $1.0 \mathrm{~mL} / \mathrm{min}, 254 \mathrm{~nm}, t_{1}=4.75 \mathrm{~min}$ (minor), $t_{2}$ $=5.55 \mathrm{~min}$ (major). $[\alpha]_{\mathrm{D}}{ }^{25}=-76.0964\left(\mathrm{c}=0.50, \mathrm{CHCl}_{3}\right) .{ }^{1} \mathrm{H} \mathrm{NMR}\left(500 \mathrm{MHz}, \mathrm{CDCl}_{3}\right) \delta 7.13(\mathrm{dd}, J$ $=8.2 \mathrm{~Hz}, J=5.4 \mathrm{~Hz}, 1 \mathrm{H}), 6.93-6.87(\mathrm{~m}, 2 \mathrm{H}), 2.86(\mathrm{t}, J=7.3 \mathrm{~Hz}, 2 \mathrm{H}), 2.61-2.50(\mathrm{~m}, 2 \mathrm{H}), 2.41-$ $2.36(\mathrm{~m}, 1 \mathrm{H}), 2.24-2.19(\mathrm{~m}, 1 \mathrm{H}), 2.11-2.07(\mathrm{~m}, 1 \mathrm{H}), 2.01-1.95(\mathrm{~m}, 2 \mathrm{H}), 1.90-1.81(\mathrm{~m}, 3 \mathrm{H}) .{ }^{13} \mathrm{C}$ $\operatorname{NMR}\left(151 \mathrm{MHz}, \mathrm{CDCl}_{3}\right) \delta 211.8,161.9(J=243.1 \mathrm{~Hz}), 147.2,138.9,125.3,114.4,111.8,62.7$, 39.6, 39.0, 37.0, 29.5, 27.1, 22.3. $[\alpha]_{\mathrm{D}}{ }^{25}=-76.0\left(\mathrm{c}=0.50, \mathrm{CHCl}_{3}\right) .{ }^{19} \mathrm{~F} \mathrm{NMR}\left(376 \mathrm{MHz}, \mathrm{CDCl}_{3}\right) \delta$

-117.02. HRMS (ESI) calculated for [M+H, $\left.\mathrm{C}_{14} \mathrm{H}_{16} \mathrm{FO}\right]^{+}$: 219.1180; found: 219.1182 .
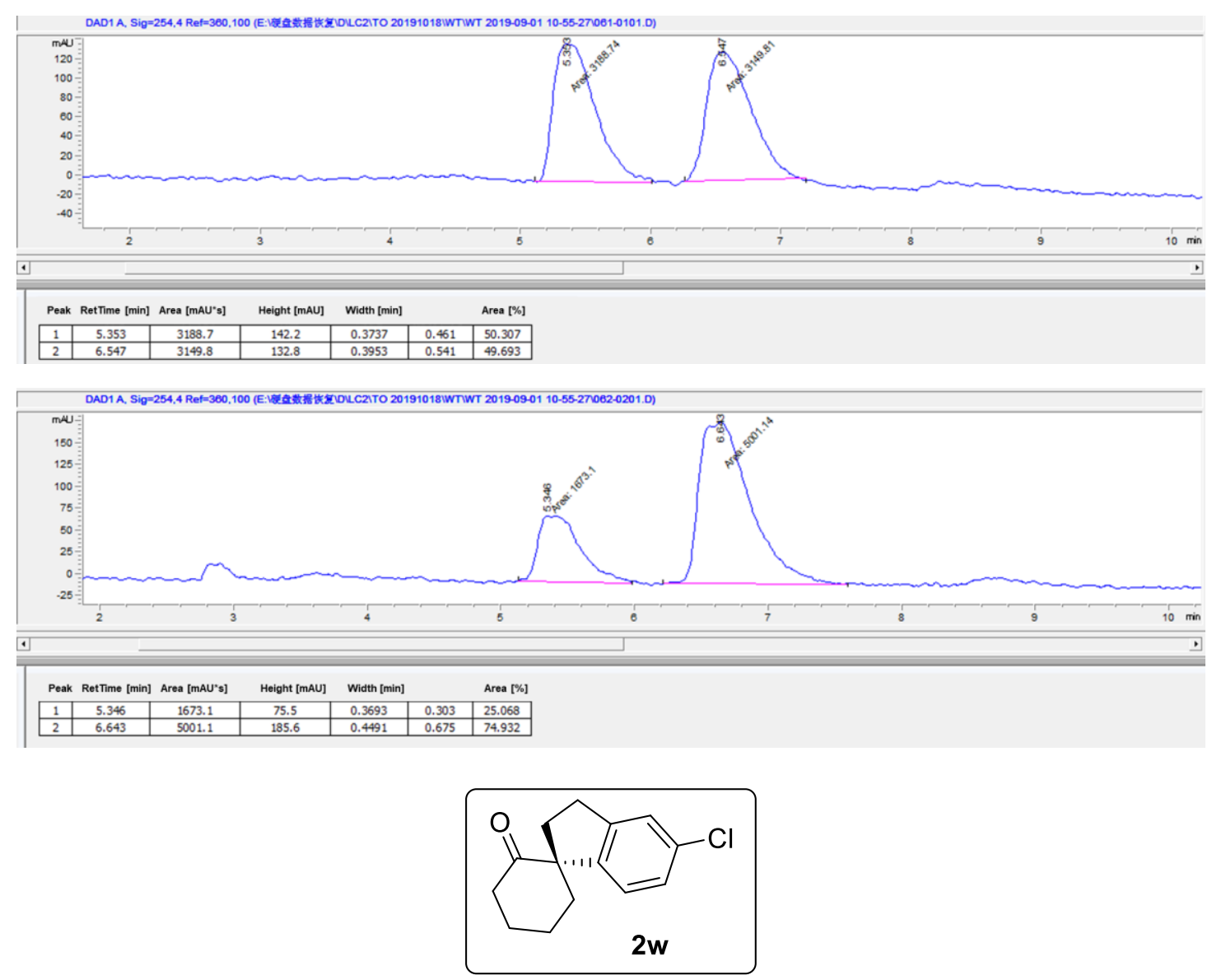

(R)-5'-Chloro-2',3'-dihydrospiro[cyclohexane-1,1'-inden]-2-one(2w): Yellow oil. 64\% yield, 74\% ee. (silica gel, eluent, petroleum ether/ethyl acetate $=5: 1$ ). Chiral HPLC conditions: chiralcel IC column, $\mathrm{n}$-hexane/ isopropanol $=85: 15$, flow rate $1.0 \mathrm{~mL} / \mathrm{min}, 290 \mathrm{~nm}, t_{1}=7.23 \mathrm{~min}$ (major), $t_{2}=$ 
$8.13 \min$ (minor). $[\alpha]_{\mathrm{D}}{ }^{25}=-88.3\left(\mathrm{c}=0.25, \mathrm{CHCl}_{3}\right) .{ }^{1} \mathrm{H} \mathrm{NMR}\left(500 \mathrm{MHz}, \mathrm{CDCl}_{3}\right) \delta 7.19(\mathrm{~d}, J=6.4$ $\mathrm{Hz}, 2 \mathrm{H}), 7.15(\mathrm{~d}, J=8.7 \mathrm{~Hz}, 1 \mathrm{H}), 2.89(\mathrm{t}, J=7.3 \mathrm{~Hz}, 2 \mathrm{H}), 2.62-2.49(\mathrm{~m}, 2 \mathrm{H}), 2.36(\mathrm{~m}, 1 \mathrm{H}), 2.20$ (m, 1H), 2.14 - 2.07 (m, 1H), 2.01 - $1.94(\mathrm{~m}, 2 \mathrm{H}), 1.93$ - $1.83(\mathrm{~m}, 3 \mathrm{H}) .{ }^{13} \mathrm{C} \mathrm{NMR}\left(126 \mathrm{MHz}, \mathrm{CDCl}_{3}\right)$ $\delta 211.8,145.5,143.6,133.0,126.5,125.8,124.7,62.1,39.5,39.0,36.4,30.1,27.0,22.3$. HRMS (ESI) calculated for $\left[\mathrm{M}+\mathrm{H}, \mathrm{C}_{14} \mathrm{H}_{16} \mathrm{ClO}\right]^{+}: 235.0884$; found: 235.0887.
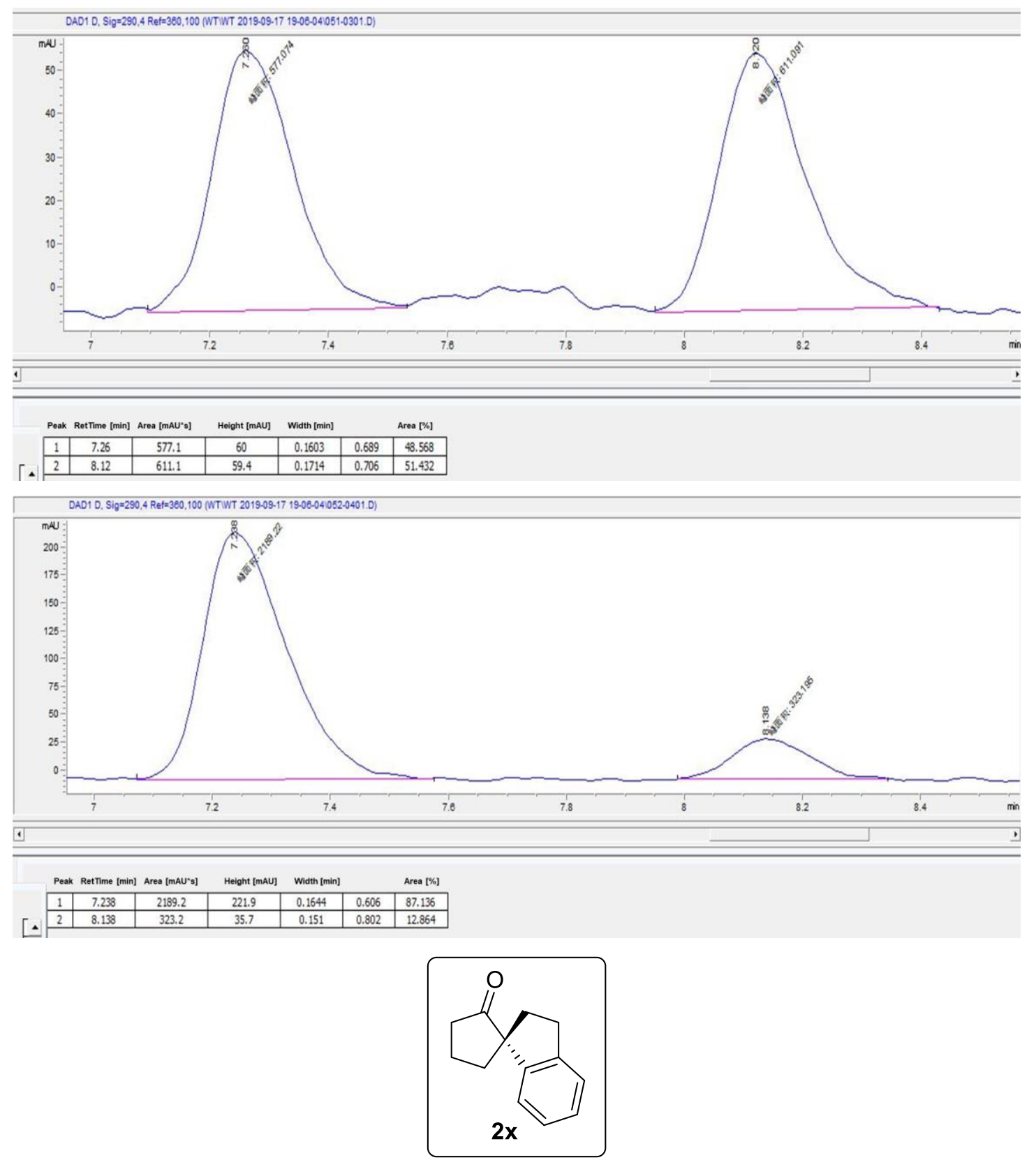

(R)-2',3'-Dihydrospiro[cyclopentane-1,1'-inden]-2-one(2x): Yellow oil. 61\% yield, 67\% ee. (silica gel, eluent, petroleum ether/ethyl acetate $=5: 1$ ). Chiral HPLC conditions: chiralcel AD-H column, $\mathrm{n}$-hexane/ isopropanol $=95: 5$, flow rate $1.0 \mathrm{~mL} / \mathrm{min}, 254 \mathrm{~nm}, t_{1}=5.80 \mathrm{~min}$ (minor), $t_{2}=$ 7.75 min (major). $[\alpha]_{\mathrm{D}^{25}}=+2.3\left(\mathrm{c}=0.70, \mathrm{CHCl}_{3}\right) .{ }^{1} \mathrm{H} \mathrm{NMR}\left(500 \mathrm{MHz}, \mathrm{CDCl}_{3}\right) \delta 7.24(\mathrm{~d}, J=0.6$ 
$\mathrm{Hz}, 1 \mathrm{H}), 7.22$ - $7.15(\mathrm{~m}, 2 \mathrm{H}), 7.04-7.01(\mathrm{~m}, 1 \mathrm{H}), 3.11-3.03(\mathrm{~m}, 1 \mathrm{H}), 2.99-2.92(\mathrm{~m}, 1 \mathrm{H}), 2.52$ $2.38(\mathrm{~m}, 2 \mathrm{H}), 2.38-2.22(\mathrm{~m}, 2 \mathrm{H}), 2.20-2.12(\mathrm{~m}, 2 \mathrm{H}), 2.07-1.94(\mathrm{~m}, 2 \mathrm{H}) ;{ }^{13} \mathrm{C} \mathrm{NMR}(126 \mathrm{MHz}$, $\left.\mathrm{CDCl}_{3}\right) \delta 221.0,145.5,144.7,127.3,126.6,124.6,122.8,61.8,37.7,37.5,37.0,30.8,19.8$. HRMS (ESI) calculated for $\left[\mathrm{M}+\mathrm{H}, \mathrm{C}_{13} \mathrm{H}_{15} \mathrm{O}\right]^{+}:$187.1117; found: 187.1121 .
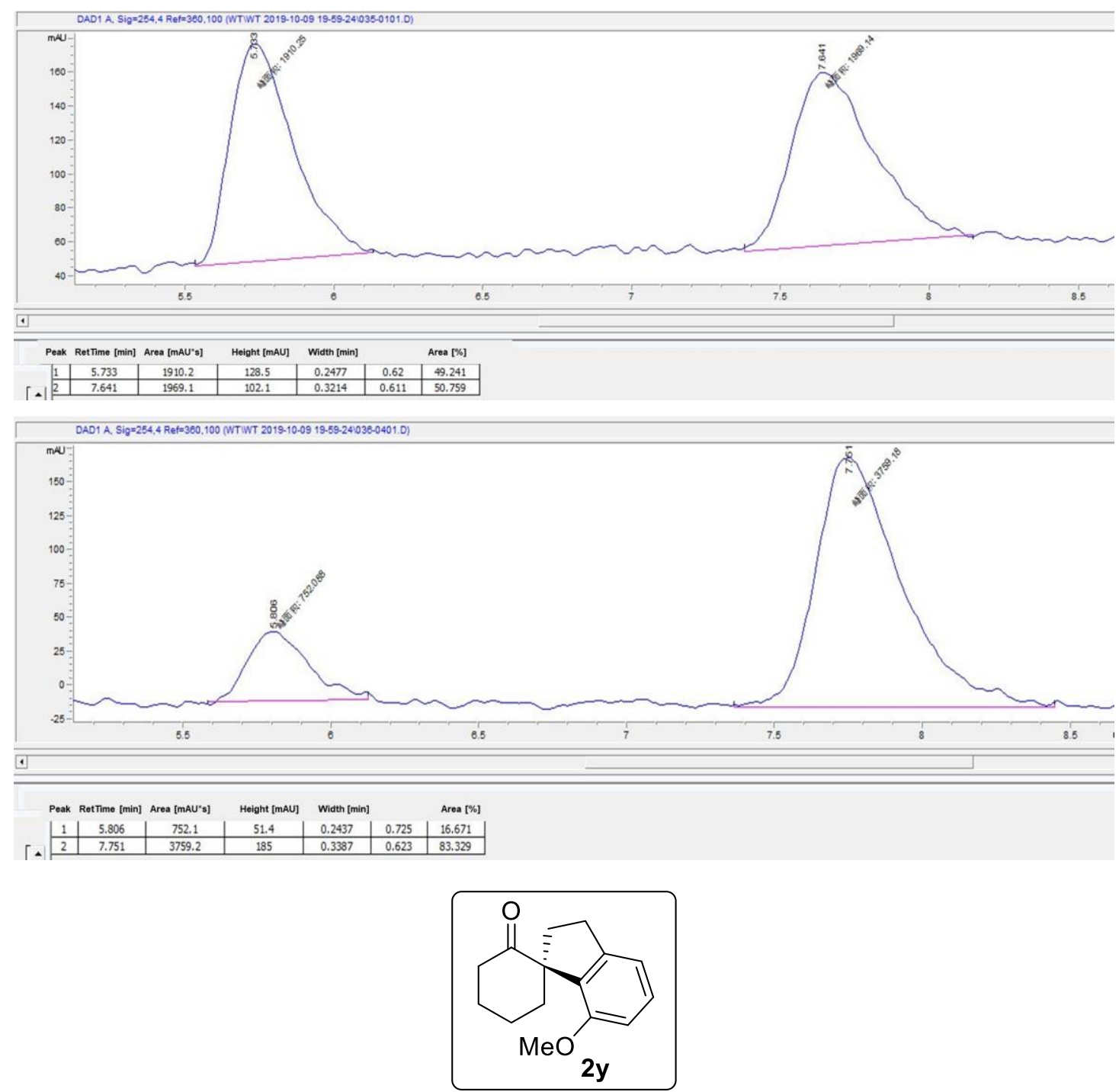

(R)-7'-Methoxy-2',3'-dihydrospiro[cyclohexane-1,1'-inden]-2-one(2y): White solid. 57\% yield, $74 \%$ ee. (silica gel, eluent, petroleum ether/ethyl acetate $=5: 1$ ). Chiral HPLC conditions: chiralcel IC column, $\mathrm{n}$-hexane/ isopropanol $=85: 5$, flow rate $1.0 \mathrm{~mL} / \mathrm{min}, 230 \mathrm{~nm}, t_{1}=9.34 \mathrm{~min}(\operatorname{minor}), t_{2}=$ 11.49 min (major). $[\alpha]_{\mathrm{D}}{ }^{25}=+34.4\left(\mathrm{c}=1.0, \mathrm{CHCl}_{3}\right){ }^{1} \mathrm{H} \mathrm{NMR}\left(500 \mathrm{MHz}, \mathrm{CDCl}_{3}\right) \delta 7.18(\mathrm{t}, J=7.8$ $\mathrm{Hz}, 1 \mathrm{H}), 6.82(\mathrm{dd}, J=7.5, J=0.7 \mathrm{~Hz}, 1 \mathrm{H}), 6.72(\mathrm{~d}, J=8.1 \mathrm{~Hz}, 1 \mathrm{H}), 3.78(\mathrm{~s}, 3 \mathrm{H}), 2.99-2.91(\mathrm{~m}$, 2H), 2.59 - $2.54(\mathrm{~m}, 1 \mathrm{H}), 2.45(\mathrm{~m}, 1 \mathrm{H}), 2.37$ - $2.30(\mathrm{~m}, 1 \mathrm{H}), 2.26(\mathrm{~m}, 1 \mathrm{H}), 2.15$ - $2.02(\mathrm{~m}, 2 \mathrm{H}), 1.88$ - $1.74(\mathrm{~m}, 4 \mathrm{H}) .{ }^{13} \mathrm{C} \mathrm{NMR}\left(126 \mathrm{MHz}, \mathrm{CDCl}_{3}\right) \delta 212.0,156.0,145.2,133.5,128.9,116.9,108.9$, 
61.8, 55.3, 39.7, 36.4, 35.8, 30.9, 25.4, 22.2. HRMS (ESI) calculated for $\left[\mathrm{M}+\mathrm{H}, \mathrm{C}_{15} \mathrm{H}_{19} \mathrm{O}_{2}\right]^{+}$: 231.1380; found: 231.1381 .

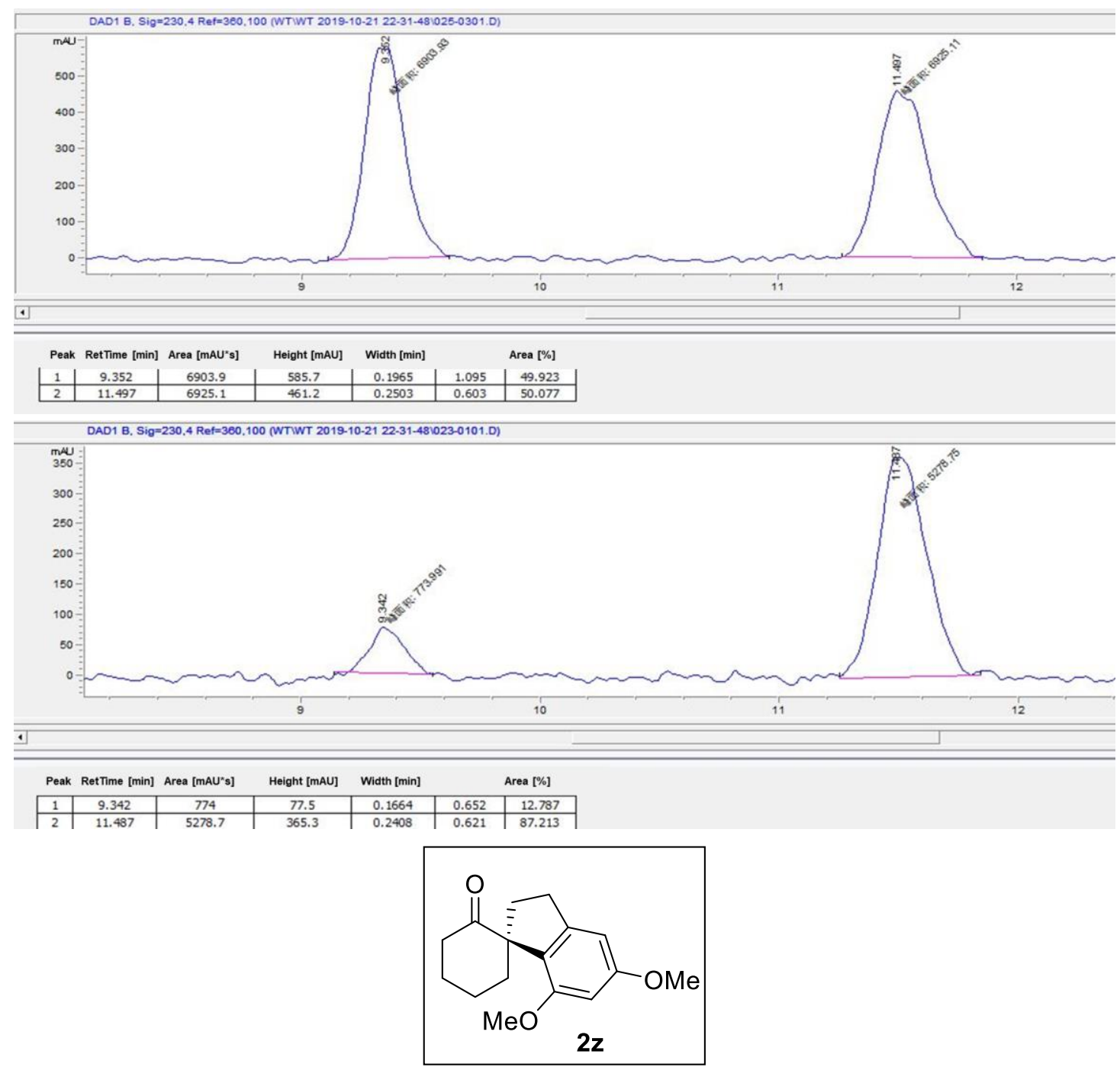

(R)-5',7'-Dimethoxy-2',3'-dihydrospiro[cyclohexane-1,1'-inden]-2-one(2z): White solid. 64\% yield, $83 \%$ ee. (silica gel, eluent, petroleum ether/ethyl acetate $=5: 1$ ). Chiral HPLC conditions: chiralcel IC column, $\mathrm{n}$-hexane/ isopropanol $=85: 15$, flow rate $1.0 \mathrm{~mL} / \mathrm{min}, 230 \mathrm{~nm}, t_{1}=15.54 \mathrm{~min}$ (major), $t_{2}=17.00 \mathrm{~min}$ (minor). $[\alpha]_{\mathrm{D}}{ }^{25}=-22.7\left(\mathrm{c}=1.0, \mathrm{CHCl}_{3}\right){ }^{1} \mathrm{H} \mathrm{NMR}\left(500 \mathrm{MHz}, \mathrm{CDCl}_{3}\right) \delta 6.35$ (s, 1H), $6.31(\mathrm{~d}, J=1.9 \mathrm{~Hz}, 1 \mathrm{H}), 3.78(\mathrm{~s}, 3 \mathrm{H}), 3.75(\mathrm{~s}, 3 \mathrm{H}), 2.90(\mathrm{~m}, 2 \mathrm{H}), 2.58-2.52(\mathrm{~m}, 1 \mathrm{H}), 2.45$ (m, 1H), 2.38 - $2.30(\mathrm{~m}, 1 \mathrm{H}), 2.25(\mathrm{~m}, 1 \mathrm{H}), 2.17$ - $2.09(\mathrm{~m}, 1 \mathrm{H}), 2.07$-2.01 (m, 1H), 1.87 - $1.71(\mathrm{~m}$, 4H). ${ }^{13} \mathrm{C}$ NMR $\left(126 \mathrm{MHz}, \mathrm{CDCl}_{3}\right) \delta 212.1,161.0,156.6,145.8,125.9,100.5,97.4,61.46,55.39$, 55.30, 39.78, 36.78, 35.57, 31.25, 25.59, 22.36. HRMS (EI) calculated for $\mathrm{C}_{16} \mathrm{H}_{20} \mathrm{O}_{3}[\mathrm{M}]^{+}: 260.1412$ found 260.1416 . 

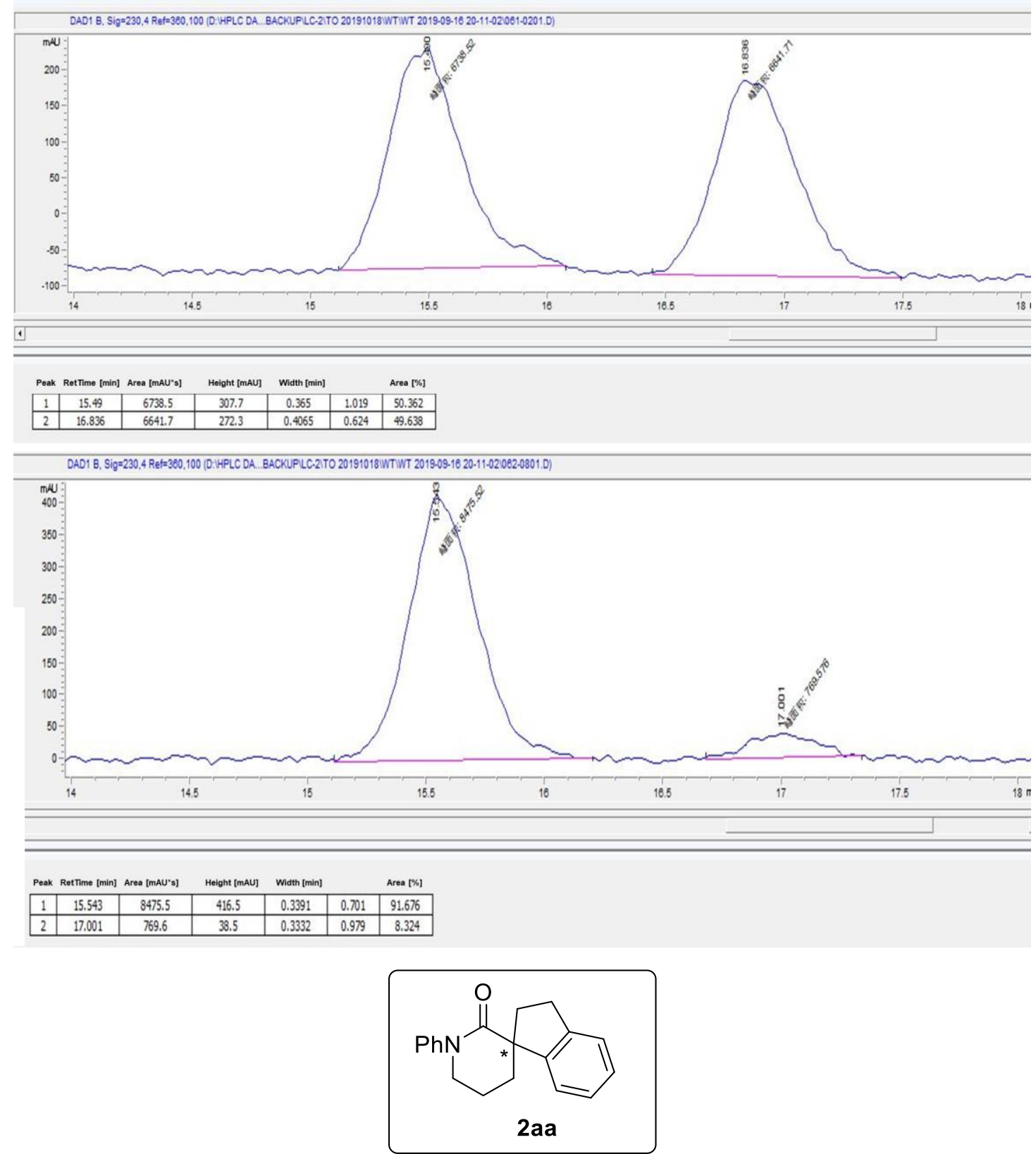

1'-Phenyl-2,3-dihydrospiro[indene-1,3'-piperidin]-2'-one(2aa): Yellow oil. 61\% yield, 68\% ee. (silica gel, eluent, petroleum ether/ethyl acetate $=5: 1$ ). Chiral HPLC conditions: chiralcel IC column, n-hexane/ isopropanol $=60: 40$, flow rate $1.0 \mathrm{~mL} / \mathrm{min}, 254 \mathrm{~nm}, t_{1}=22.62 \mathrm{~min}$ (minor), $t_{2}=27.15$ $\min$ (major). $[\alpha]_{\mathrm{D}}{ }^{25}=+6.8\left(\mathrm{c}=1.0, \mathrm{CHCl}_{3}\right) .{ }^{1} \mathrm{H}$ NMR $\left(500 \mathrm{MHz}, \mathrm{CDCl}_{3}\right) \delta 7.40-7.36(\mathrm{~m}, 2 \mathrm{H}), 7.32$ - $7.27(\mathrm{~m}, 3 \mathrm{H}), 7.26-7.21(\mathrm{~m}, 3 \mathrm{H}), 7.20-7.19(\mathrm{~m}, 1 \mathrm{H}), 3.83(\mathrm{t}, J=5.9 \mathrm{~Hz}, 2 \mathrm{H}), 3.73-3.61(\mathrm{~m}$, 1H), $3.10(\mathrm{~m}, 1 \mathrm{H}), 3.05-2.98(\mathrm{~m}, 1 \mathrm{H}), 2.91-2.86(\mathrm{~m}, 1 \mathrm{H}), 2.20-2.13(\mathrm{~m}, 2 \mathrm{H}), 2.11-2.05(\mathrm{~m}$, $2 \mathrm{H}) .{ }^{13} \mathrm{C} \mathrm{NMR}\left(126 \mathrm{MHz}, \mathrm{CDCl}_{3}\right) \delta 173.78,148.00,143.88,143.77,128.96,127.28,126.48,126.32$, 
$126.14,124.78,123.37,55.75,52.10,38.70,34.49,30.72,20.57$. HRMS (ESI) calculated for $\left[\mathrm{M}+\mathrm{Na}, \mathrm{C}_{19} \mathrm{H}_{19} \mathrm{NNaO}\right]^{+}: 300.1364$; found: 300.1359 .
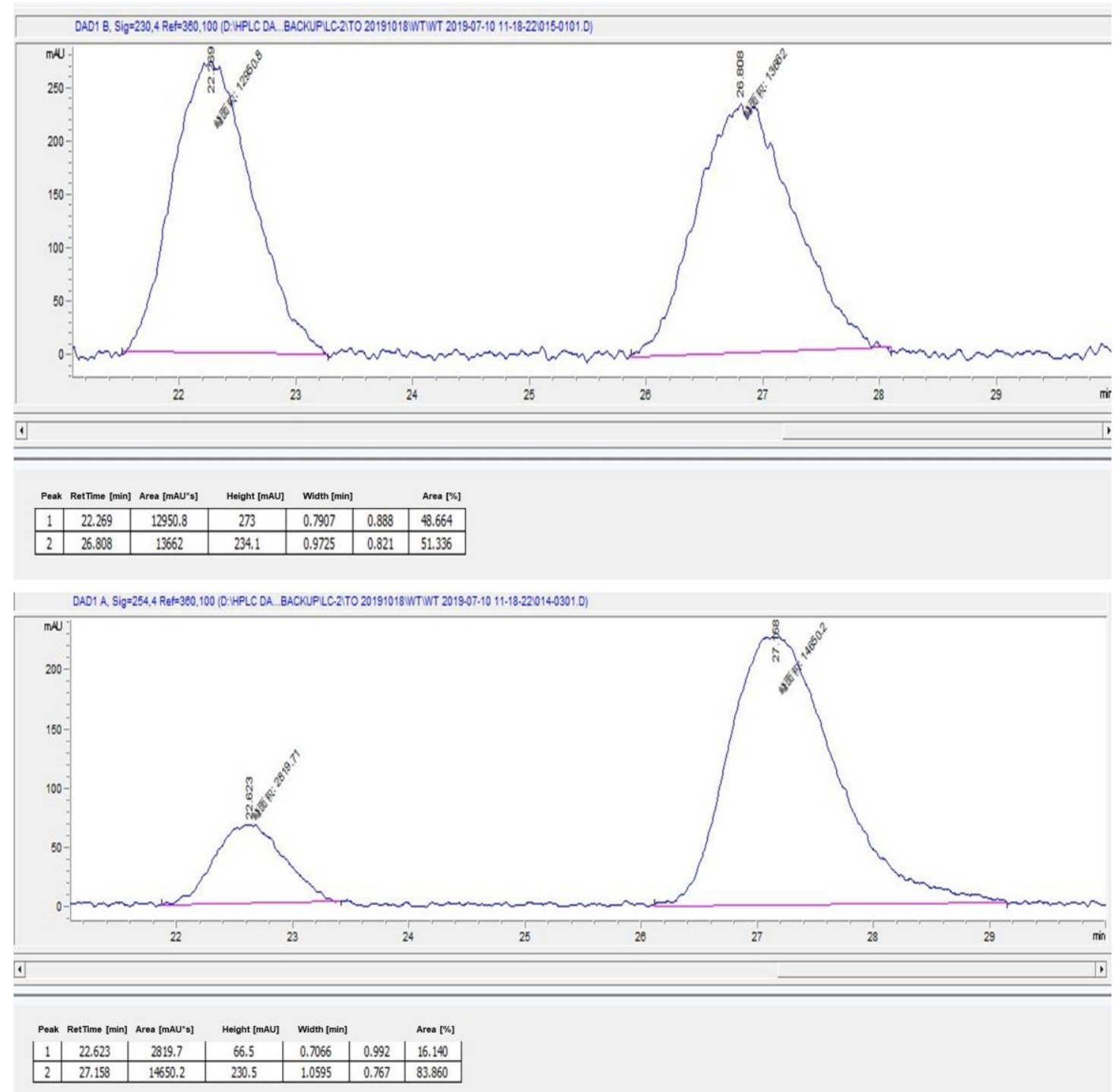

\section{Gram-scale reaction}

To a mixture of compound 1a (1g, 3.05 mmol, 1.0 equiv.), $\mathrm{NaO} t \mathrm{Bu}$ (440 mg, $4.58 \mathrm{mmol}, 1.5$ equiv.), $\mathrm{H}_{2} \mathrm{O}(0.17 \mathrm{ml}, 9.15 \mathrm{mmol}, 3.0$ equiv. $)$ in THF $(5 \mathrm{ml})$ was charged with $\mathrm{Pd}_{2}(\mathrm{dba})_{3}(0.015 \mathrm{mmol}, 14$ $\mathrm{mg}, 0.5 \mathrm{~mol} \%)$, $\mathbf{L 9}(0.036 \mathrm{mmol}, 26 \mathrm{mg}, 1.2 \mathrm{~mol} \%)$ under $\mathrm{N}_{2}$ atmosphere. The mixture was stirred at $70{ }^{\circ} \mathrm{C}$ for $48 \mathrm{~h}$ and then cooled to room temperature. The mixture was directly purified by flash chromatography on silica gel to afford the desired product $\mathbf{2 a}$ ( $745 \mathrm{mg}, 98 \%$ yield, $92 \%$ ee).

\section{Synthetic application}




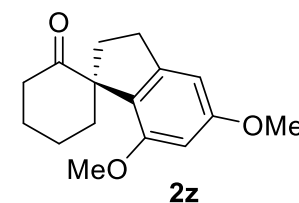

$2 z$

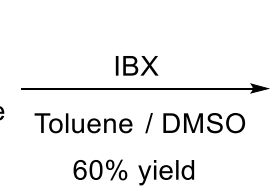

$60 \%$ yield

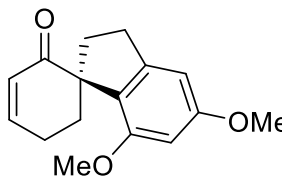

(1) $\mathrm{H}_{2} \mathrm{O}_{2}$ $\mathrm{NaOH}, \mathrm{MeOH}$

(2) $\mathrm{NH}_{2} \mathrm{NH}_{2} \cdot \mathrm{H}_{2} \mathrm{O}$

(3) DMP $60 \%$ yield

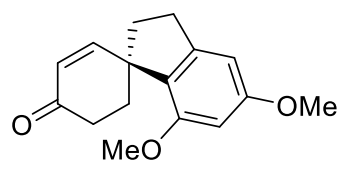

4

Synthesis of 3: To a solution of ketone (2z) (40 mg, $0.15 \mathrm{mmol})$ in DMSO-toluene (3:1, $3 \mathrm{ml}, 0.2$ $\mathrm{M}$ with respect to IBX) was added 2-iodoxybenzoic acid (IBX, $65 \mathrm{mg}, 0.23 \mathrm{mmol}, 1.5$ equiv.). The solution was heated to $80{ }^{\circ} \mathrm{C}$ (oiling bath) for $12 \mathrm{~h}$. The reaction mixture was cooled to room temperature and poured into a mixture of $\mathrm{Et}_{2} \mathrm{O}(20 \mathrm{~mL})$ and water $(30 \mathrm{~mL})$. The white solid residue was collected by filtration and washed with $\mathrm{Et}_{2} \mathrm{O}(50 \mathrm{~mL})$. The organic layer was washed with water $(3 \times 20 \mathrm{~mL})$, an aqueous saturated $\mathrm{NaHCO}_{3}$ solution $(3 \times 20 \mathrm{~mL})$, an aqueous $1 \mathrm{~N} \mathrm{NaOH}$ solution $(10 \mathrm{~mL})$, brine $(20 \mathrm{~mL})$, dried over $\mathrm{Na}_{2} \mathrm{SO}_{4}$, and filtered followed by removal of solvent in vacuo. The residue was purified using flash column chromatography (silica gel, petroleum ether /ethyl acetate, 10:1) to give the enone $\mathbf{3}$ (24 $\mathrm{mg}, 60 \%$ yield) as a white solid.

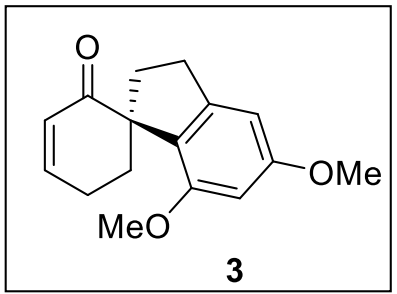

(S)-5',7'-Dimethoxy-2',3'-dihydrospiro[cyclohexane-1,1'-inden]-3-en-2-one(3): White solid. 60\% yield, $80 \%$ ee. ( silica gel, eluent, petroleum ether/ethyl acetate $=3: 1$ ). Chiral HPLC conditions: chiralcel AD-H column, $\mathrm{n}$-hexane/ isopropanol $=80: 20$, flow rate $1.0 \mathrm{~mL} / \mathrm{min}, 254 \mathrm{~nm}, t_{1}=8.11$ $\min \left(\right.$ major), $t_{2}=11.19 \min$ (minor). $[\alpha]_{\mathrm{D}}{ }^{25}=-10.4\left(\mathrm{c}=0.936, \mathrm{CHCl}_{3}\right) .{ }^{1} \mathrm{H} \mathrm{NMR}\left(400 \mathrm{MHz}, \mathrm{CDCl}_{3}\right)$ $\delta 6.89(\mathrm{dd}, J=10.1,1.6 \mathrm{~Hz}, 1 \mathrm{H}), 6.39(\mathrm{~s}, 1 \mathrm{H}), 6.29(\mathrm{~d}, J=1.8 \mathrm{~Hz}, 1 \mathrm{H}), 5.95(\mathrm{~d}, J=10.1 \mathrm{~Hz}, 1 \mathrm{H}), 3.80$ (s, 3H), $3.72(\mathrm{~s}, 3 \mathrm{H}), 3.03-2.88(\mathrm{~m}, 2 \mathrm{H}), 2.29-2.62(\mathrm{~m}, 4 \mathrm{H}), 2.07-1.98(\mathrm{~m}, 1 \mathrm{H}), 1.90$ - $1.95(\mathrm{~m}$, 1H). ${ }^{13} \mathrm{C}$ NMR $\left(126 \mathrm{MHz}, \mathrm{CDCl}_{3}\right) \delta 201.56,161.17,156.38,149.06,146.47,129.29,126.18,100.73$, 97.40, 57.87, 55.42, 55.25, 34.10, 31.47, 31.25, 23.84. HRMS (ESI) calculated for $\left[\mathrm{M}+\mathrm{Na}, \mathrm{C}_{16} \mathrm{H}_{18} \mathrm{NaO}_{3}\right]^{+}:$281.1148; found: 281.1153 . 

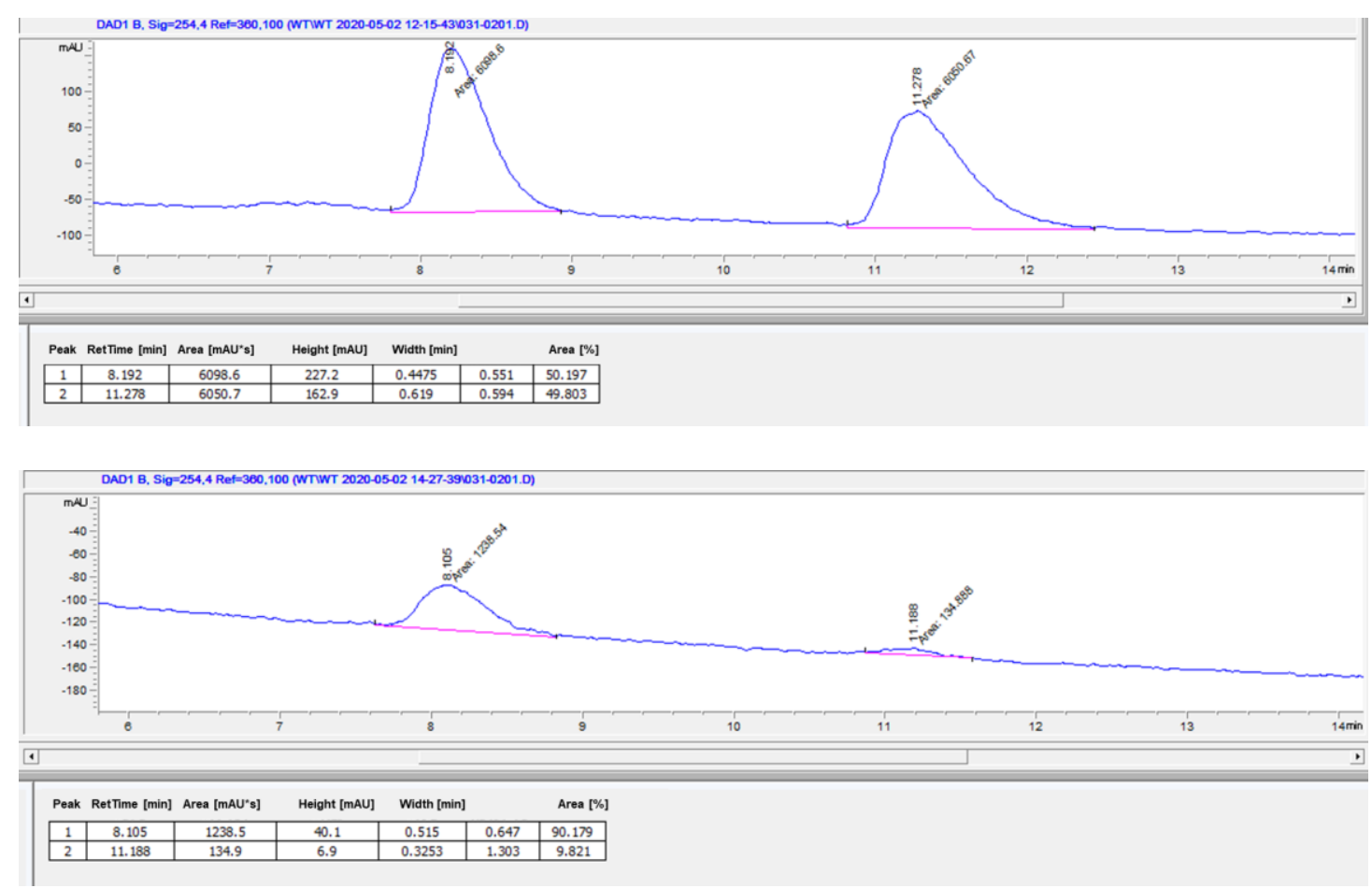

Synthesis of 4: In a dry, nitrogen flushed, $25-\mathrm{mL}$, round-bottomed flask containing a magnetic stirring bar was placed enone 3 ( $60 \mathrm{mg}, 0.23 \mathrm{mmol}$ ) and $6 \mathrm{~mL}$ of methanol. The solution was cooled to $0{ }^{\circ} \mathrm{C}$ and then stirred while $1 \mathrm{M} \mathrm{NaOH}$ solution $(0.3 \mathrm{ml}, 0.3 \mathrm{mmol})$ was added. After slowly adding $30 \% \mathrm{H}_{2} \mathrm{O}_{2}$ solution $\left(0.3 \mathrm{ml}, 1.98 \mathrm{mmol}, 3\right.$ equiv.), the reaction mixture was stirred at $0{ }^{\circ} \mathrm{C}$ for $15 \mathrm{~min}$ and then stirred at room temperature for hours. The reaction mixture was directly extracted with $3 \times 20 \mathrm{~mL}$ of ether. The ether layers were combined, washed with water $(3 \times 20 \mathrm{~mL})$ and brine $(1 \times 20 \mathrm{~mL})$, and then dried over $\mathrm{Na}_{2} \mathrm{SO}_{4}$. Removal of solvent left a residue of the epoxy ketone sufficiently pure for use in the next step.

In a 25-mL flask (nitrogen atmosphere) was placed epoxy ketone and a magnetic stirring bar. Upon adding hydrazine hydrate $(3 \mathrm{ml})$ at room temperature, the reaction was heated slowly to reflux $\left(120{ }^{\circ} \mathrm{C}\right.$ oiling bath) and kept at reflux for $15 \mathrm{~min}$. After cooling, the reaction mixture was diluted with $15 \mathrm{~mL}$ of ether, and then the solution was washed with water $(3 \times 20 \mathrm{~mL})$ and brine $(1 \times 20$ $\mathrm{mL}$ ) and then dried over $\mathrm{Na}_{2} \mathrm{SO}_{4}$. Filtration and removal of solvent yielded allyl alcohol.

1,1,1-Triacetoxy-1,1-dihydro-1,2-benziodoxol-3(1H)-one (DMP, $216 \mathrm{mg}, 0.51 \mathrm{mmol}, 2.14$ equiv.) was added in one portion to a stirred solution of the alcohol in dichloromethane $(3.50 \mathrm{~mL})$ at room temperature. The reaction solution was stirred at $\mathrm{rt}$ for $40 \mathrm{~min}$, then was diluted with ethyl acetate $(10 \mathrm{~mL})$, water $(3 \mathrm{~mL})$, saturated aqueous sodium thiosulfate solution $(3 \mathrm{~mL})$, and saturated aqueous sodium bicarbonate solution $(2 \mathrm{~mL})$. The resulting biphasic mixture was stirred vigorously until 
both layers were clear and colorless. The layers were separated and the aqueous layer was extracted with two $20 \mathrm{~mL}$ portions of ethyl acetate. The combined organic layers were dried over $\mathrm{Na}_{2} \mathrm{SO}_{4}$ and filtered. The filtrate was concentrated. The residue obtained was purified by flash-column chromatography on silica gel (eluent, petroleum ether/ethyl acetate $=5: 1$ ). to give the enone $\mathbf{4}$ as white solid (35 mg, 60\%).

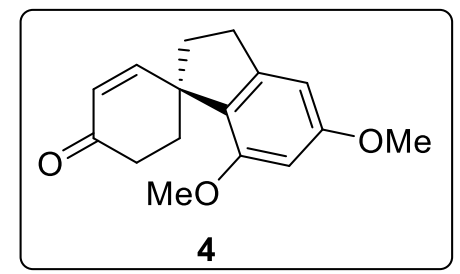

(S)-5',7'-Dimethoxy-2',3'-dihydrospiro[cyclohexane-1,1'-inden]-2-en-4-one(4): White solid. (silica gel, eluent, petroleum ether/ethyl acetate $=5: 1$ ), 60\% yield. $78 \%$ ee. Chiral HPLC conditions: chiralcel IA column, $\mathrm{n}$-hexane/ isopropanol = 90: 10, flow rate $1.0 \mathrm{~mL} / \mathrm{min}, 280 \mathrm{~nm}, t_{1}=10.32 \mathrm{~min}$ (minor), $t_{2}=11.06 \min$ (major). $[\alpha]_{\mathrm{D}}{ }^{25}=-19.2\left(\mathrm{c}=0.25, \mathrm{CHCl}_{3}\right) .{ }^{1} \mathrm{H} \mathrm{NMR}\left(500 \mathrm{MHz}, \mathrm{CDCl}_{3}\right) \delta$ $7.00-6.94(\mathrm{~m}, 1 \mathrm{H}), 6.38(\mathrm{~s}, 1 \mathrm{H}), 6.30(\mathrm{~d}, J=1.6 \mathrm{~Hz}, 1 \mathrm{H}), 6.10(\mathrm{dd}, J=10.1,1.6 \mathrm{~Hz}, 1 \mathrm{H}), 3.78(\mathrm{~s}$, $3 \mathrm{H}), 3.71(\mathrm{~s}, 3 \mathrm{H}), 2.97-2.91(\mathrm{~m}, 2 \mathrm{H}), 2.69-2.60(\mathrm{~m}, 1 \mathrm{H}), 2.46-2.54(\mathrm{~m}, 1 \mathrm{H}), 2.41-2.36(\mathrm{~m}, 1 \mathrm{H})$, 2.23- $2.10(\mathrm{~m}, 1 \mathrm{H}), 2.07-2.13(\mathrm{~m}, 1 \mathrm{H}), 187-1.83(\mathrm{~m}, 1 \mathrm{H}) .{ }^{13} \mathrm{C} \mathrm{NMR}\left(151 \mathrm{MHz}, \mathrm{CDCl}_{3}\right) \delta 200.1$, 161.2, 158.7, 156.9, 146.0, 127.6, 126.4, 100.8, 97.1, 55.5, 55.1, 48.4, 35.6, 35.2, 31.3, 30.9. HRMS (ESI) calculated for $\left[\mathrm{M}+\mathrm{H}, \mathrm{C}_{16} \mathrm{H}_{19} \mathrm{O}_{3}\right]^{+}: 259.1329$; found: 259.1333 .

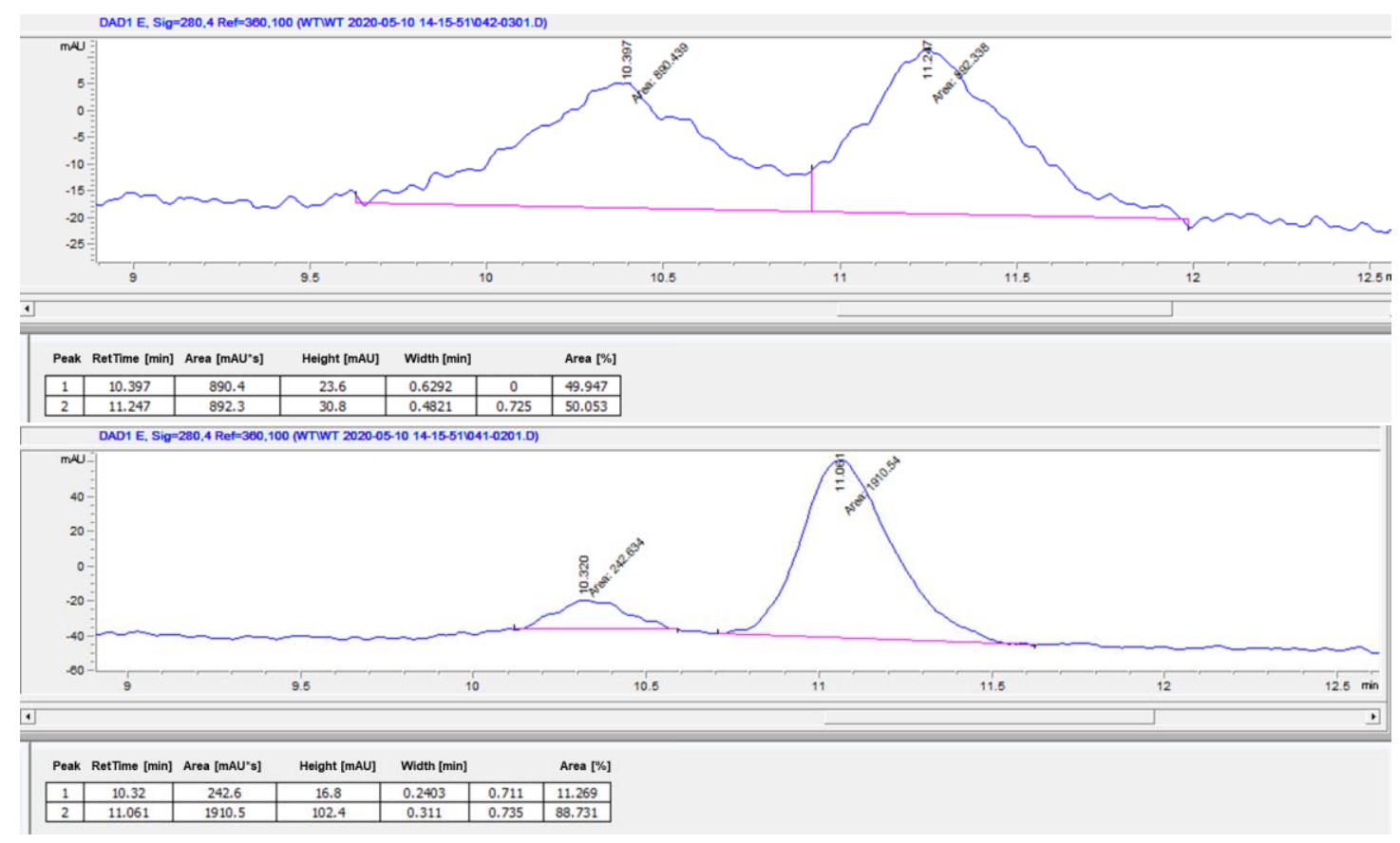




\section{References:}

(1) Tang, W.; Capacci, A. G.; Wei, X.; Li, W.; White, A.; Patel, N. D.; Savoie, J.; Gao, J. J.; Rodriguez, S.; Haddad, B. N.; Lu, B. Z.; Krishnamurthy, D.; Yee, N. K.; Senanayake, C. H. A General and Special Catalyst for Suzuki-Miyaura Coupling Processes. Angew. Chem., Int. Ed. 2010, 49, 5879-5883.

(2) Tang, W.; Patel, N. D.; Xu, G.; Xu, X.; Savoie, J.; Ma, S.; Hao, M. H.; Kehipeddy, S.; Capacci, A. G.; Wei, X.; Zhang, Y.; Gao, J. J.; Li, W.; Rodriguez, S.; Lu, B. Z.; Yee, N. K.; Senanayake, C. H. Efficient Chiral Monophosphorus Ligands for Asymmetric Suzuki-Miyaura Coupling Reactions. Org. Lett. 2012, 14, 2258-2261.

(3) Xu, G.; Fu, W.; Liu, G.; Senanayake, C. H.; Tang, W. Efficient Syntheses of Korupensamines A, B and Michellamine B by Asymmetric Suzuki-Miyaura Coupling Reactions. J. Am. Chem. Soc. 2014, 136, 570-573.

(4) Xiong, W.; Xu, G.; Yu, X.; Tang, W. P-Chiral Monophosphorus Ligands for Asymmetric Copper-Catalyzed Allylic Alkylation. Organometallics 2019, 38, 4003-4013.

(5) (a) Grigg, R. D.; Hovein, R. V.; Schomaker, J. M. Copper-Catalyzed Recycling of Halogen Activating Groups via 1,3-Halogen Migration. J. Am. Chem. Soc. 2012, 134, 16131-16134. (b) Hu, Y.; Xie, Y.; Shen, Z.; Huang, H. Palladium - Catalyzed Ring - Forming Aminoalkenylation of Alkenes with Aldehydes Initiated by Intramolecular Aminopalladation. Angew. Chem., Int. Ed. 2017, 56, 2473-2477. (c) Percy, J. M.; H. Emerson, Fyfe, J. W. B.; Kennedy, A. R.; Maciuk, S.; Orr, D.; Rathouska, L.; Redmond, J. M.; Wilson, P. G. Modular Construction of Fluoroarenes from a New Difluorinated Building Block by Cross - Coupling/Electrocyclisation/Dehydrofluorination Reactions. Chemistry - A European Journal. 2016, 22, 12166-12175.

(6) Rodriguez, A. L.; Bunlaksananusorn, T.; Knochel, P. Potassium tert-Butoxide Catalyzed Addition of Carbonyl Derivatives to Styrenes. Org. Lett. 2000, 2, 3285-3287.

(7) Fan, L.; Takizawa, S.; Takeuchi, Y.; Takenaka, K.; Sasai, H. Pd-catalyzed enantioselective intramolecular $\alpha$-arylation of $\alpha$-substituted cyclic ketones: facile synthesis of functionalized chiral spirobicycles. Org. Biomol. Chem. 2015, 13, 4837-4840. 
9.NMR and HPLC spectra

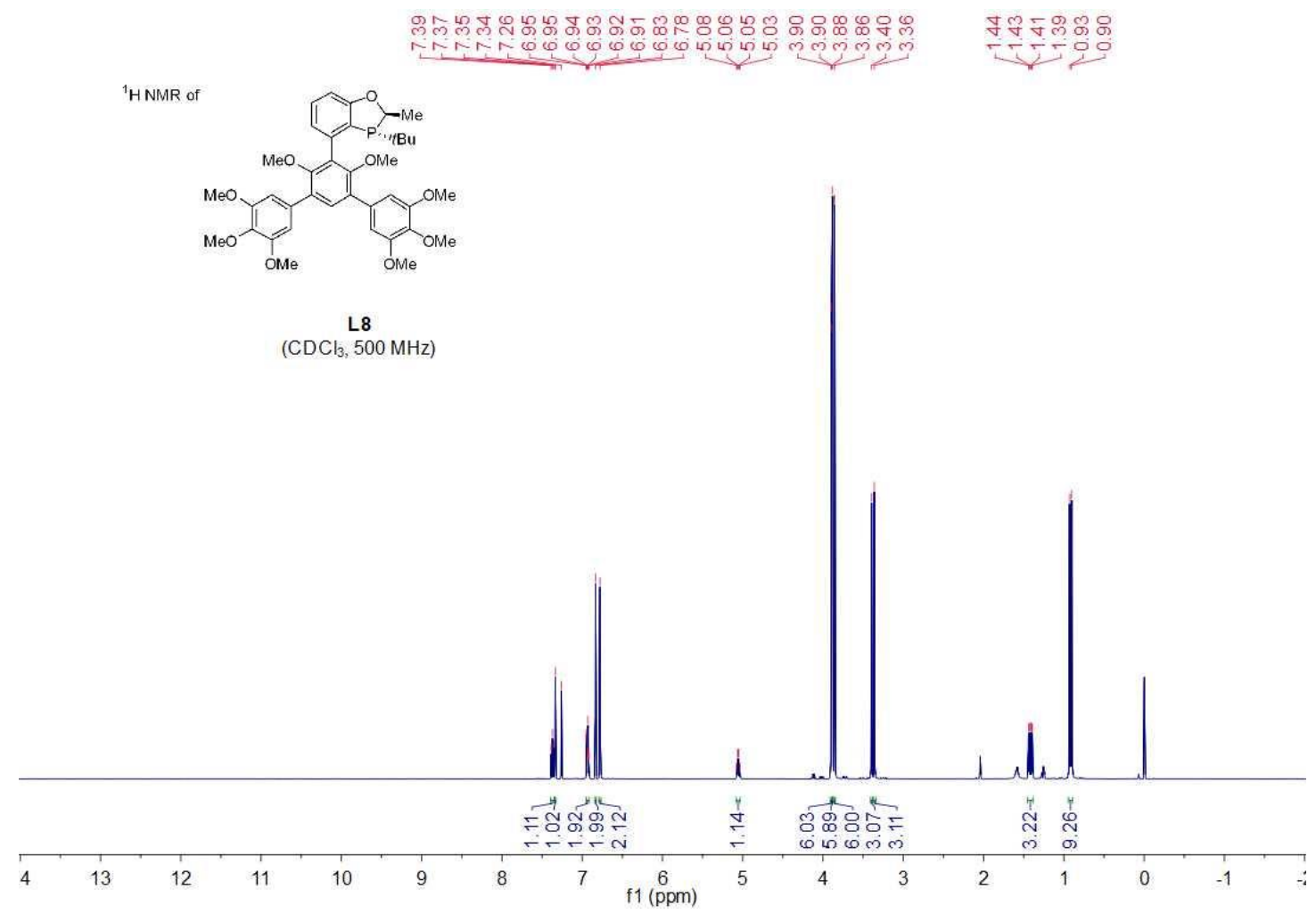




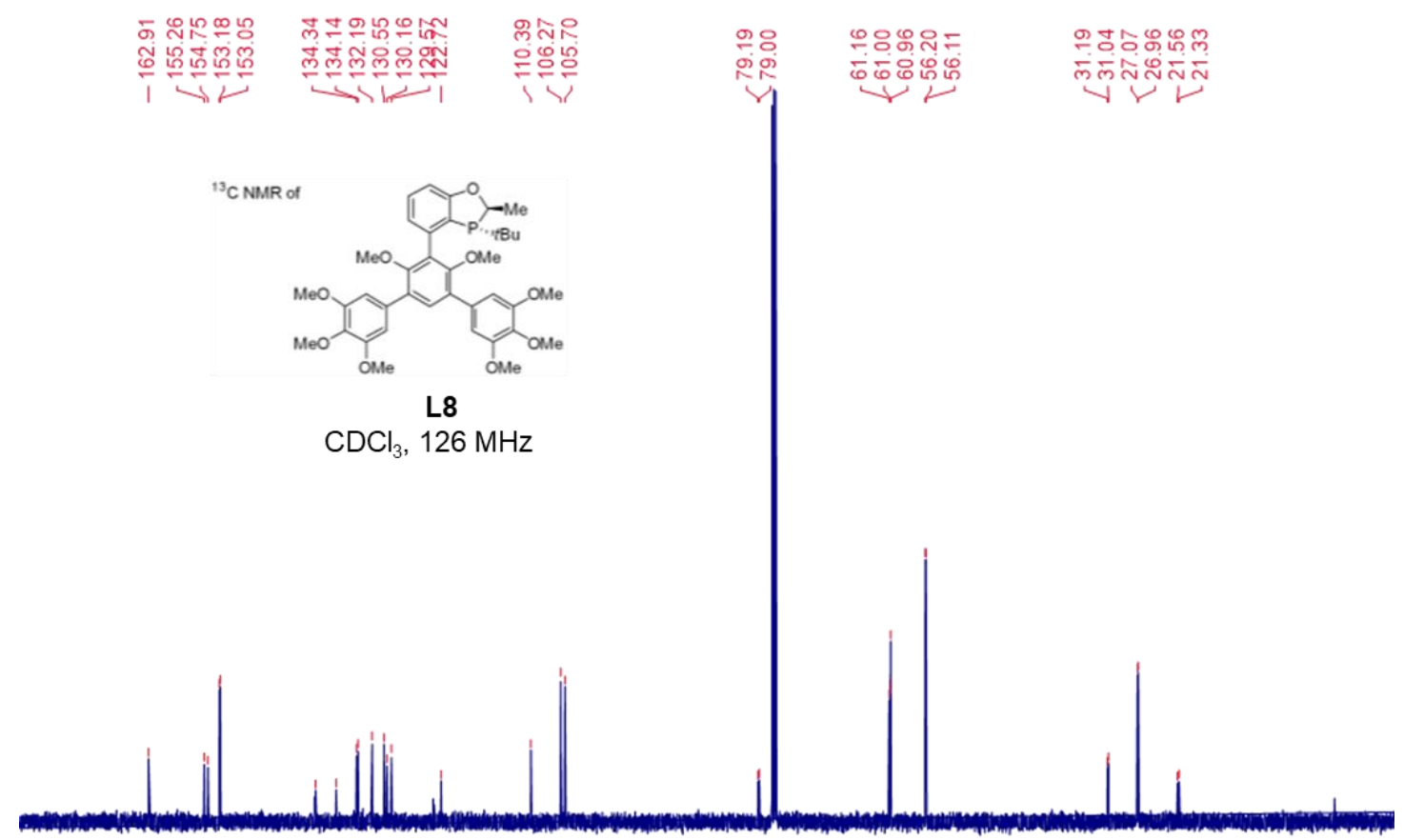

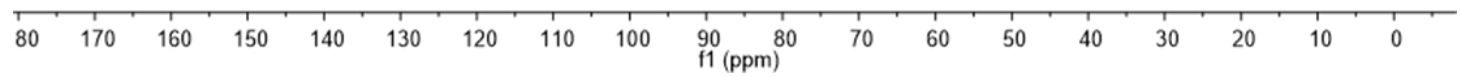

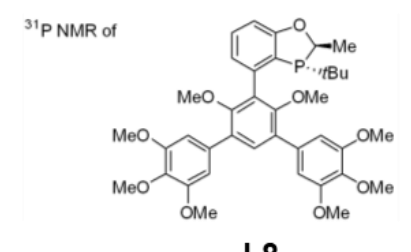

L8

$162 \mathrm{MHz}, \mathrm{CDCl}_{3}$

\begin{tabular}{|c|c|c|c|c|c|c|c|c|c|c|c|c|c|c|c|c|c|}
\hline 190 & 170 & 150 & 130 & 110 & 90 & $\begin{array}{l}8070 \\
\text { f1 (ppm) }\end{array}$ & 60 & 50 & 40 & 30 & 20 & 10 & 0 & -10 & -20 & -30 & -40 \\
\hline
\end{tabular}


${ }^{1} \mathrm{H}$ NMR of

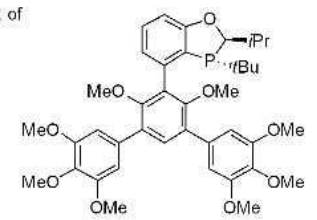

L9

$\left(\mathrm{CDCl}_{3}, 500 \mathrm{MHz}\right)$

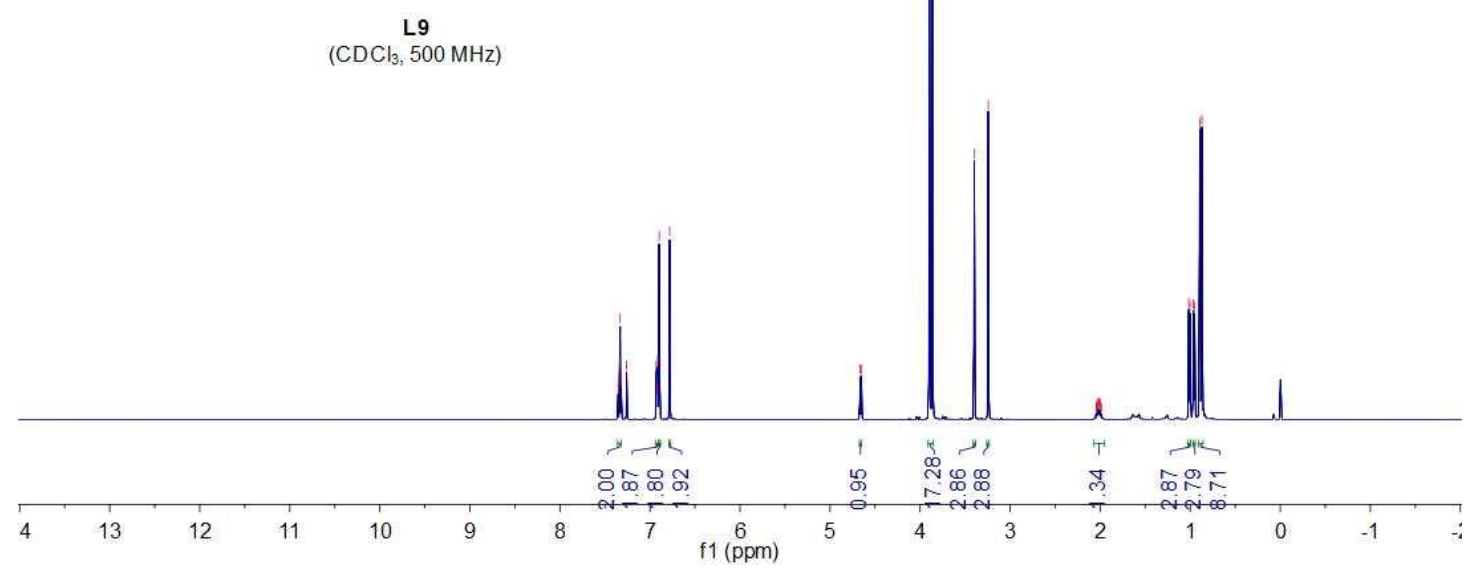

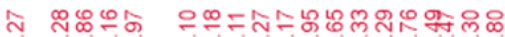

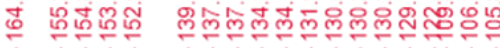

年

${ }^{13} \mathrm{C}$ NMR of

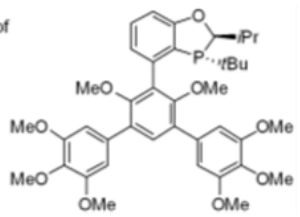

L9

$126 \mathrm{MHz} \mathrm{CDCl}_{3}$

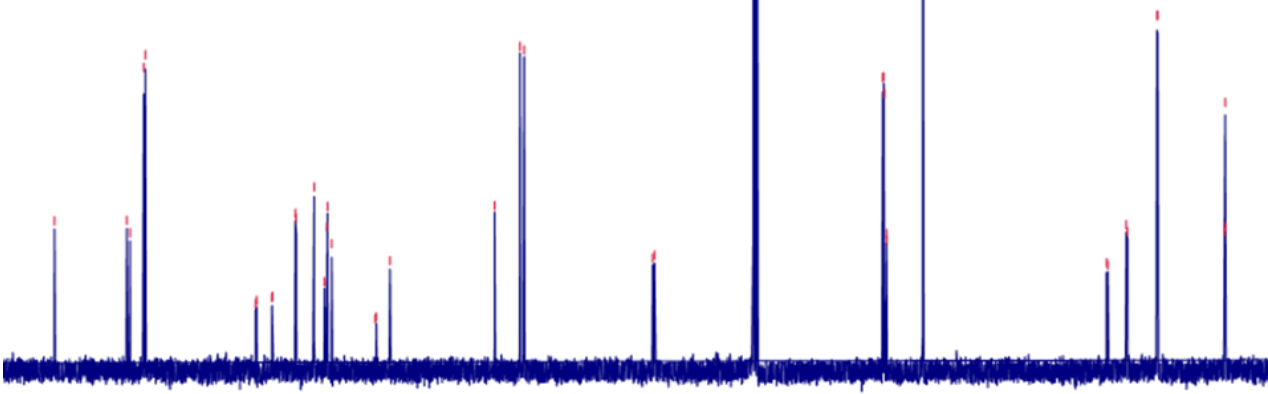

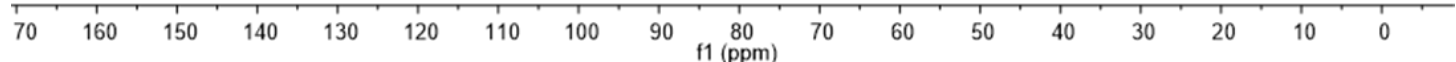




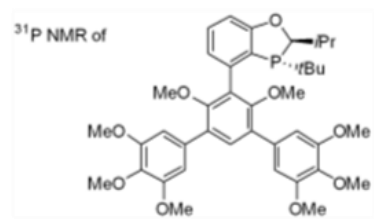

L9

$162 \mathrm{MHz}, \mathrm{CDCl}_{3}$

\begin{tabular}{|c|c|c|c|c|c|c|c|c|c|c|c|c|c|c|c|c|}
\hline 190 & 170 & 150 & 130 & 110 & 90 & $\begin{array}{l}8070 \\
11(\mathrm{ppm})\end{array}$ & 60 & 50 & 40 & 30 & 20 & 10 & 0 & -10 & -2 & $\begin{array}{lll}0 & -30 & -40\end{array}$ \\
\hline
\end{tabular}

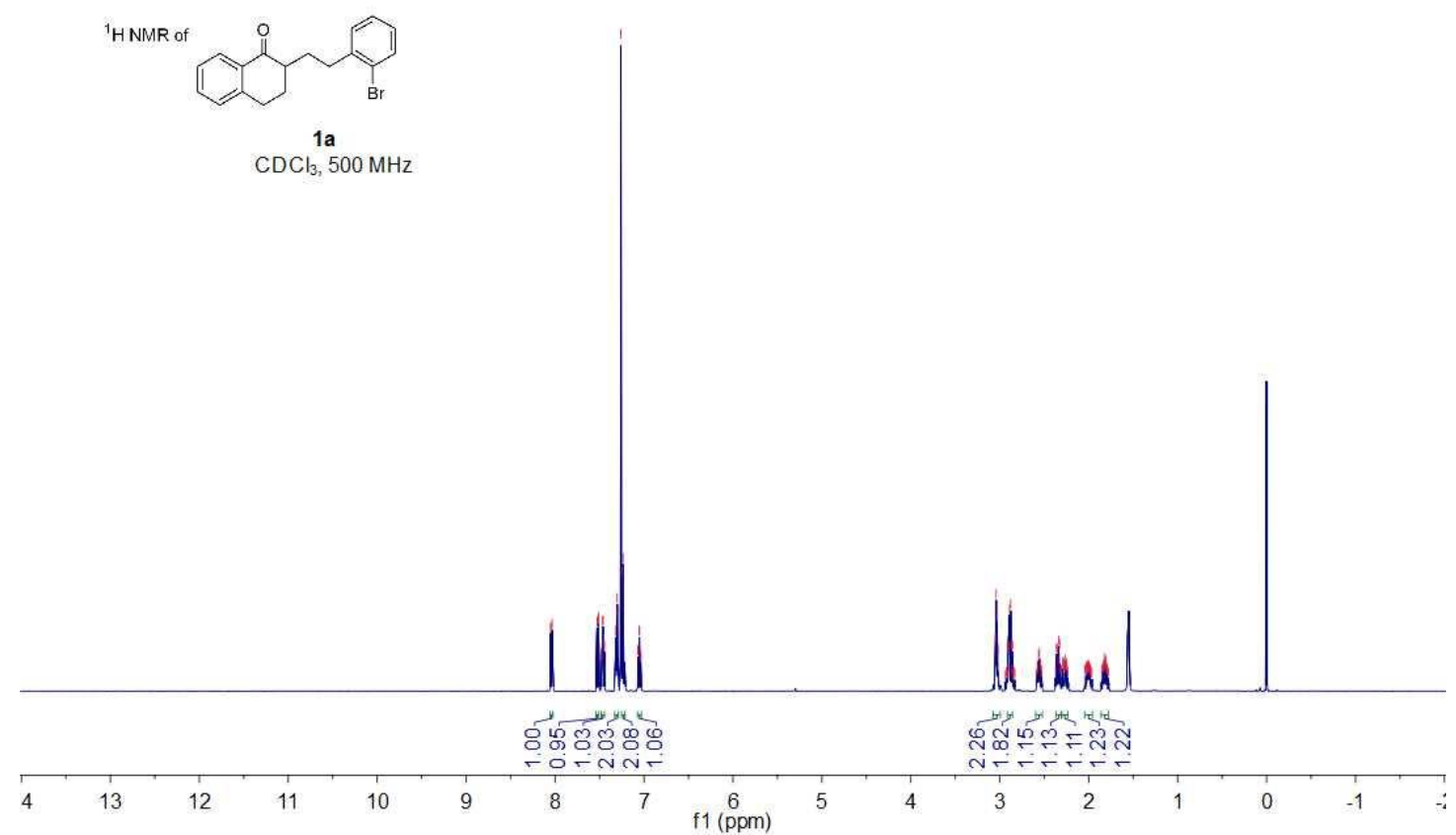


${ }^{13} \mathrm{C} \mathrm{NMR}$ of

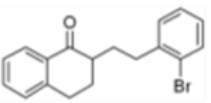

1a

$126 \mathrm{MHz}, \mathrm{CDCl}_{3}$

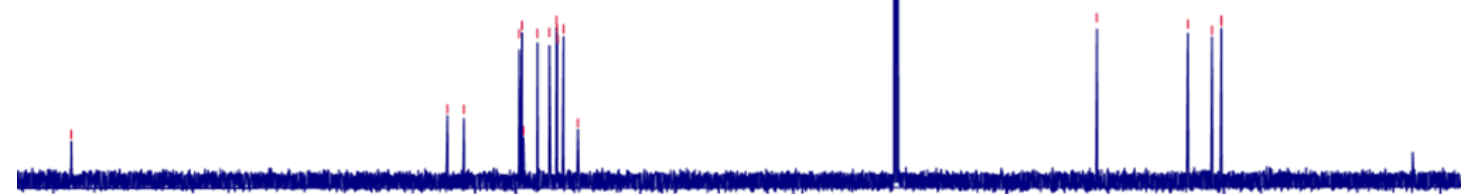

$\begin{array}{lllllllllllllllllllllllllll}1 & 1 & 190 & 180 & 170 & 160 & 150 & 140 & 130 & 120 & 110 & 100 & 90 & 80 & 70 & 60 & 50 & 40 & 30 & 20 & 10 & 0\end{array}$

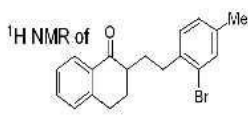

16

$\mathrm{CDCl}_{3}, 500 \mathrm{MHz}$

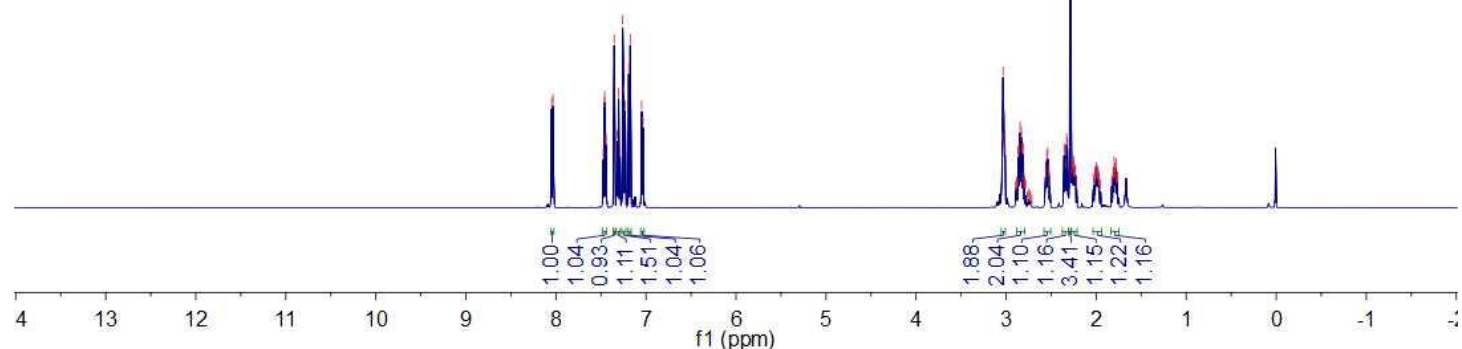


${ }^{13} \mathrm{C}$ NMR of<smiles>Cc1ccc(CCCC2CCc3ccccc3C2=O)c(Br)c1</smiles>

1b

$\mathrm{CDCl}_{3}, 126 \mathrm{MHz}$

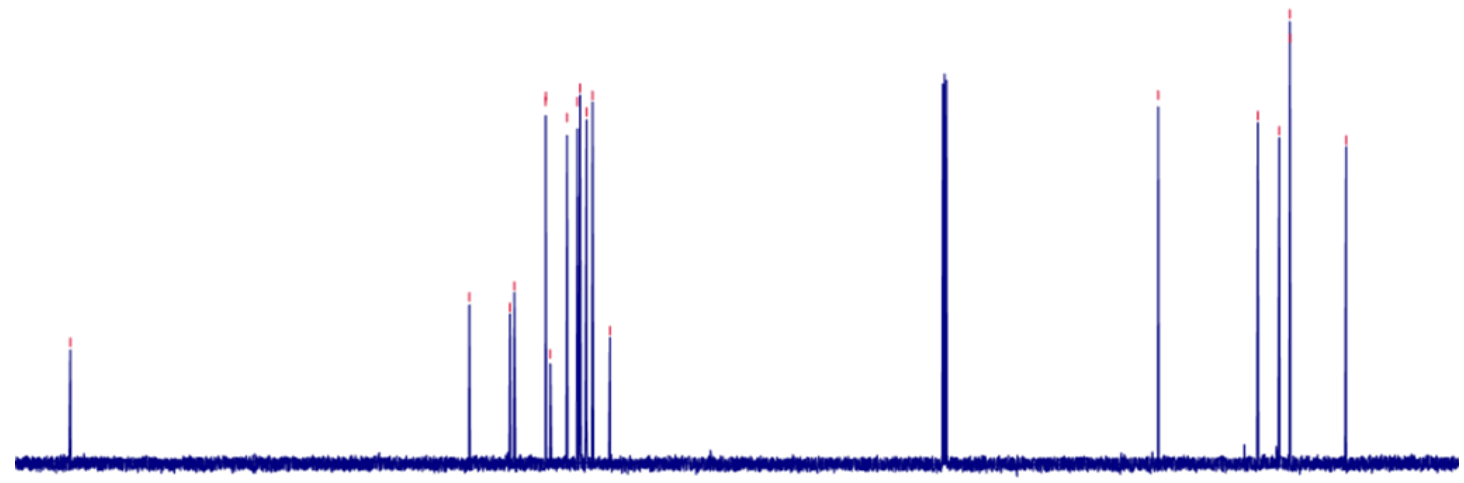

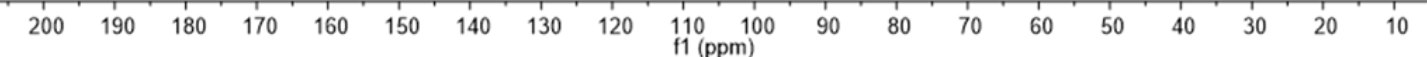

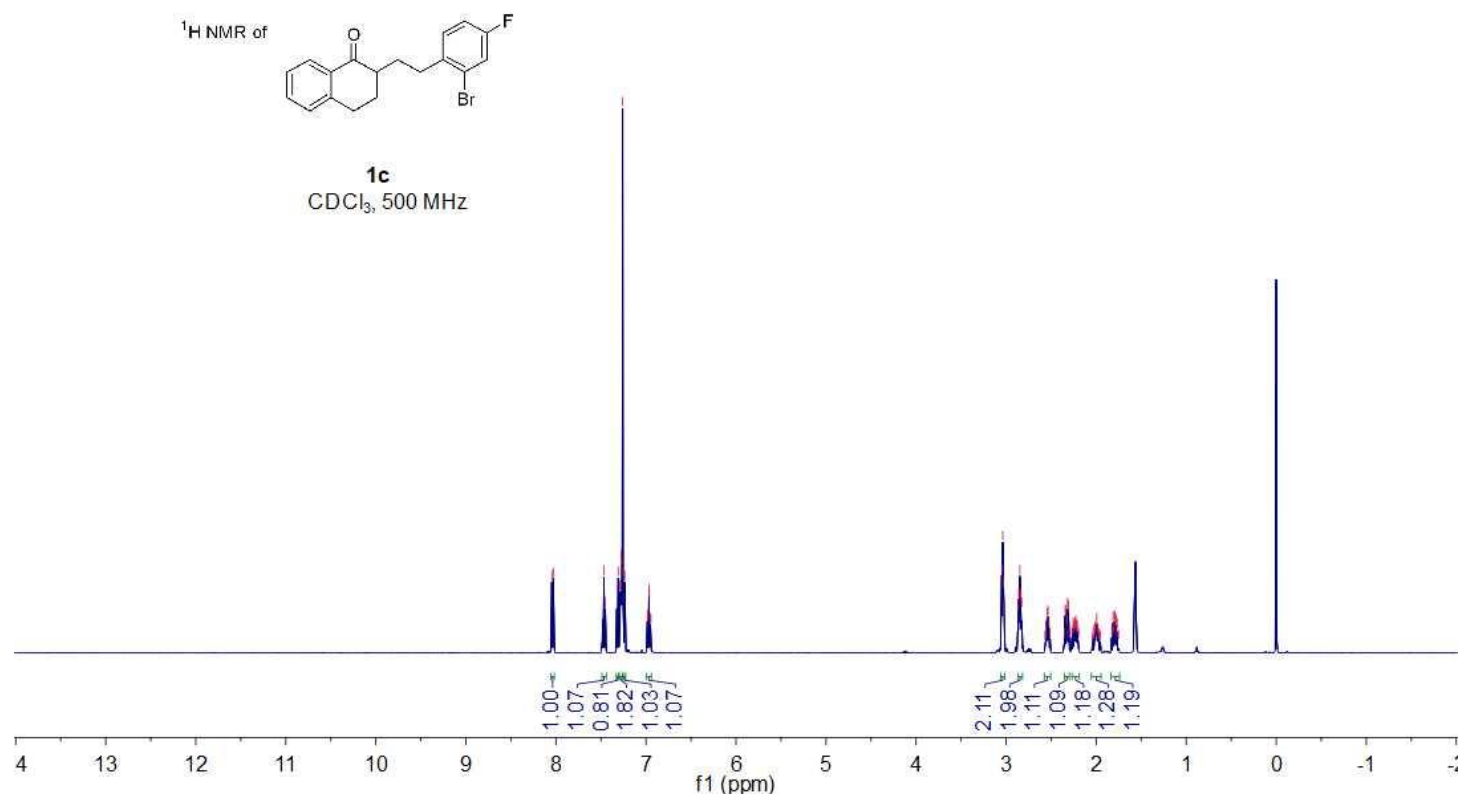


${ }^{13} \mathrm{C}$ NMR of

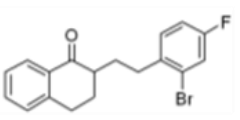

$1 \mathrm{c}$ $\mathrm{CDCl}_{3}, 126 \mathrm{MHz}$

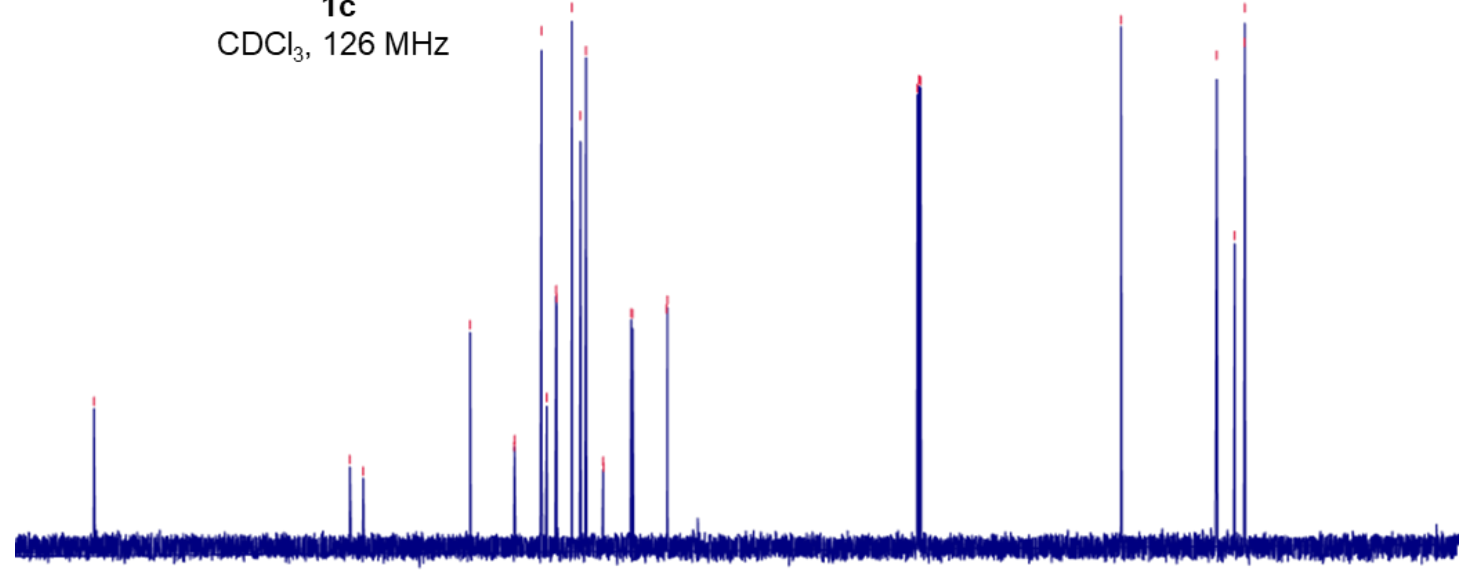

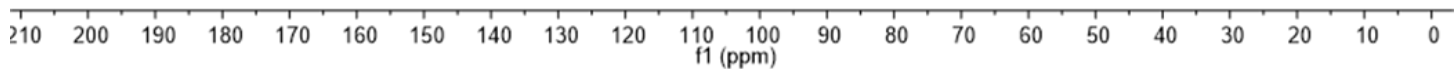

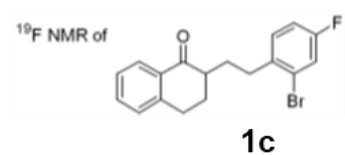

$\mathrm{CDCl}_{3}, 376 \mathrm{MHz}$

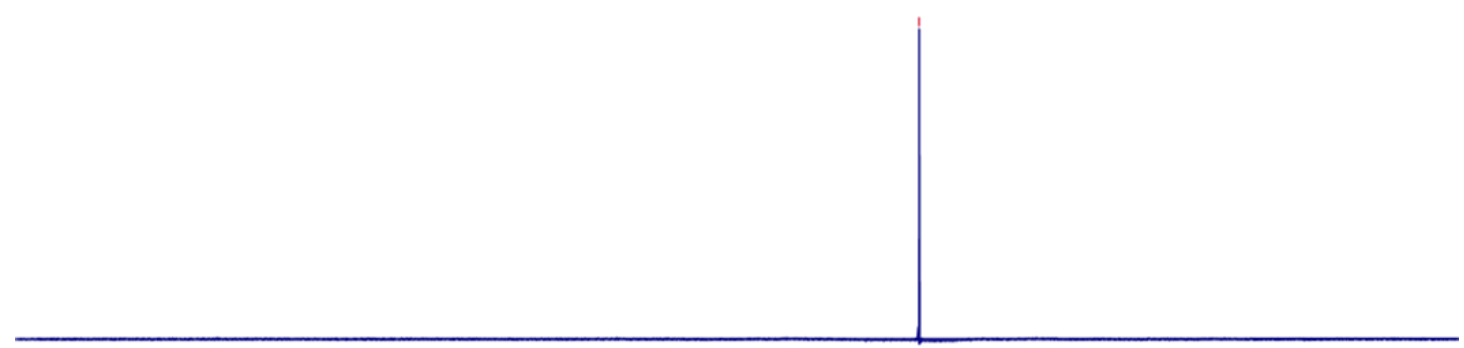

$\begin{array}{lllllllllllll}30 & 20 & 10 & 0 & -10 & -20 & -30 & -40 & -50 & -60 & -70 & -80 & -90\end{array}$ 
${ }^{1}$ H NMR of

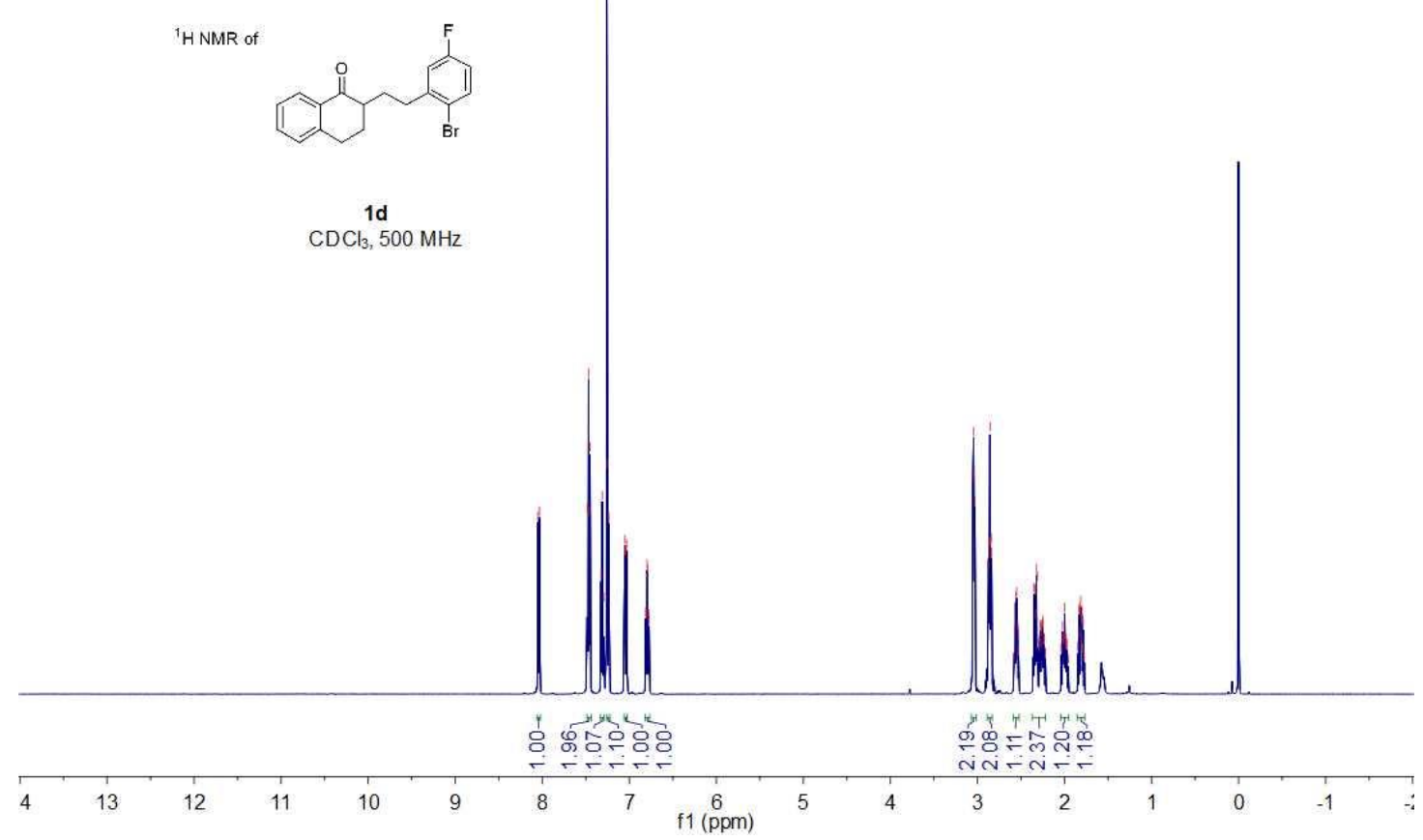

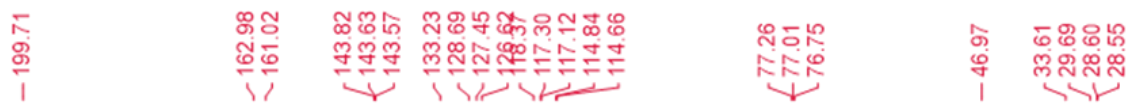

${ }^{13} \mathrm{C} \mathrm{NMR}$ of

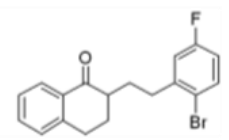

$1 d$ $\mathrm{CDCl}_{3}, 126 \mathrm{MHz}$

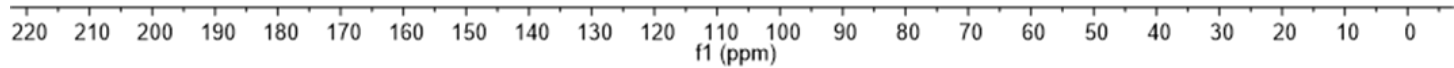




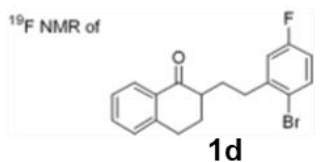

$\mathrm{CDCl}_{3}, 376 \mathrm{MHz}$

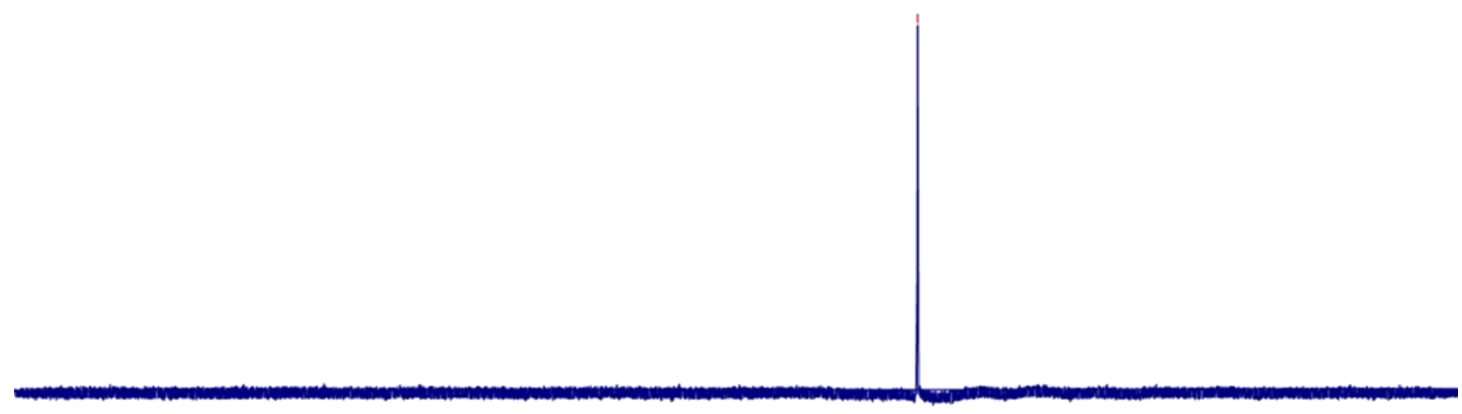

\begin{tabular}{lllllllllllllllllll}
\hline & 20 & 10 & 0 & -10 & -20 & -30 & -40 & -50 & -60 & -70 & -80 & -90 & -110 & -130 & -150 & -170 & -190
\end{tabular}

${ }^{1} \mathrm{H}$ NMR of

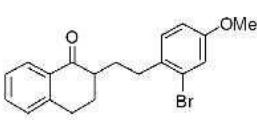

$1 e$

$\mathrm{CDCl}_{3}, 500 \mathrm{MHz}$

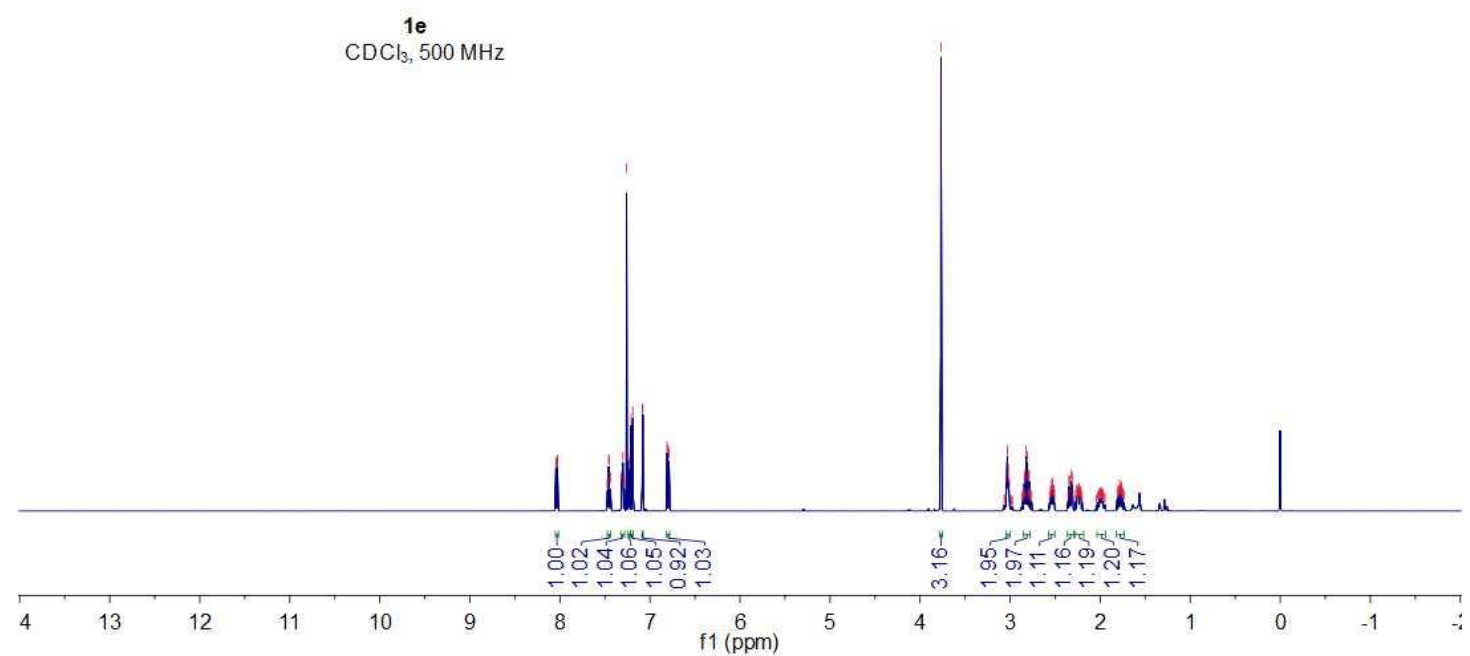




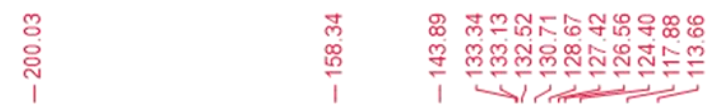

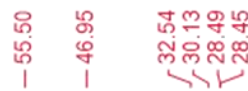

${ }^{13} \mathrm{C}$ NMR of

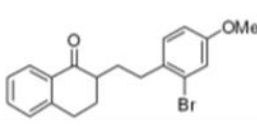

$1 \mathrm{e}$

$\mathrm{CDCl}_{3}, 126 \mathrm{MHz}$

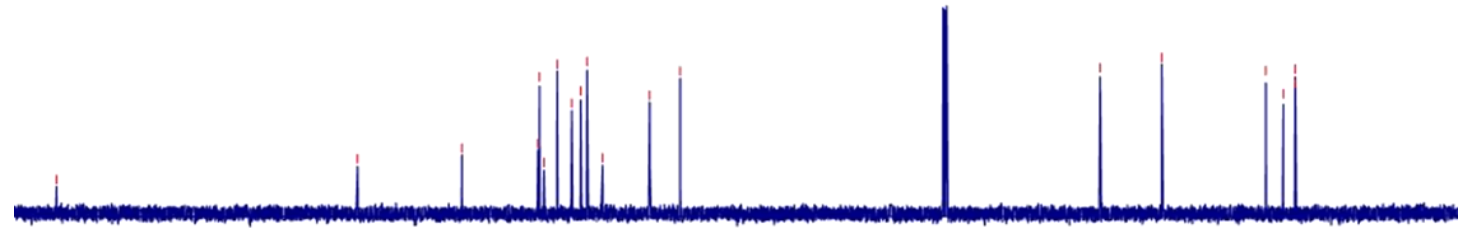

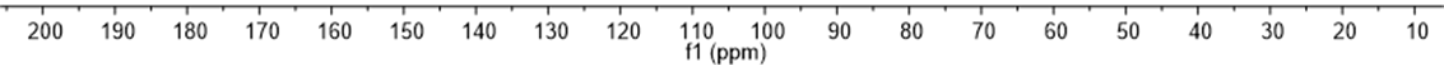

${ }^{1}$ HNMR of

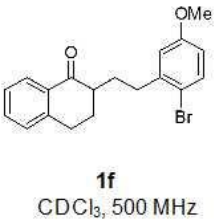

$\mathrm{CDCl}_{3}, 500 \mathrm{MHz}$

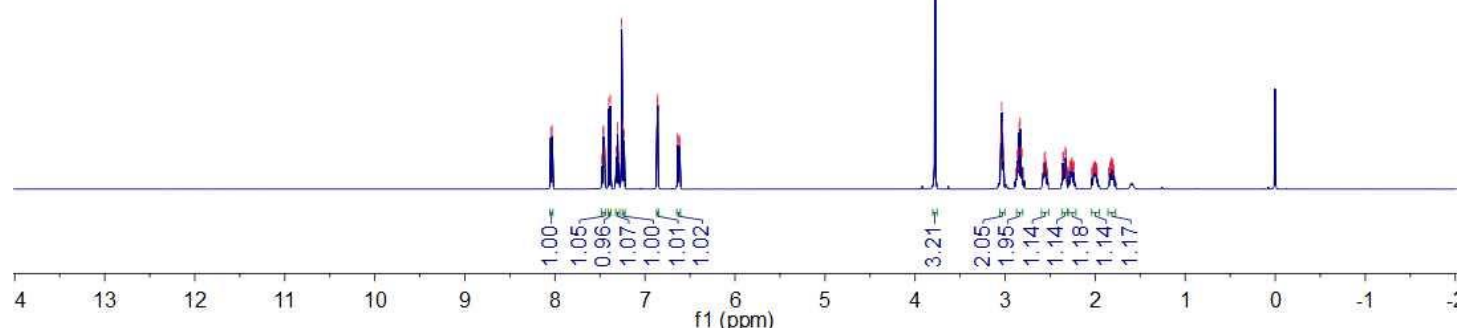



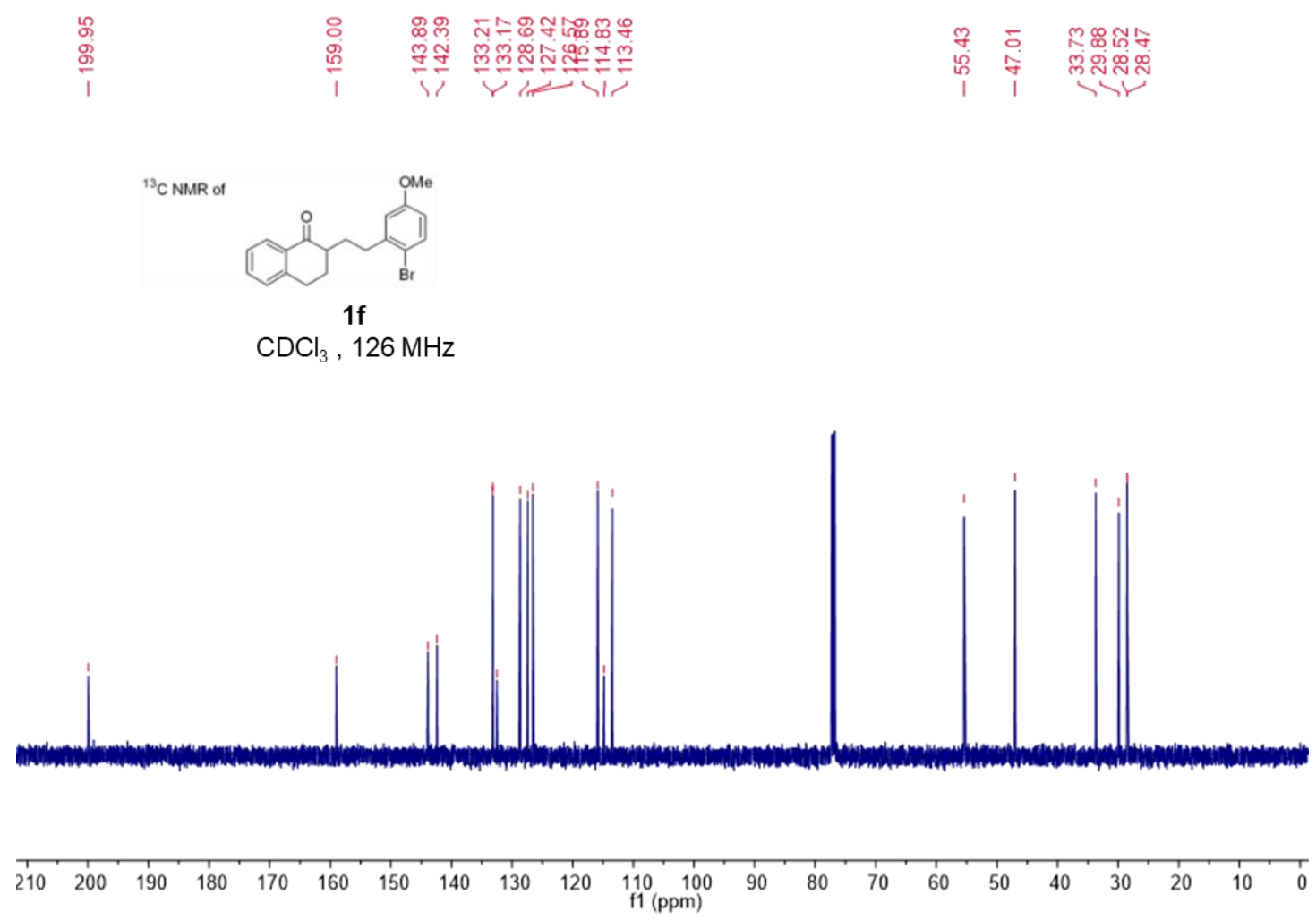

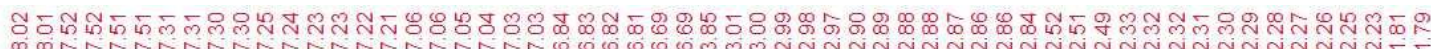

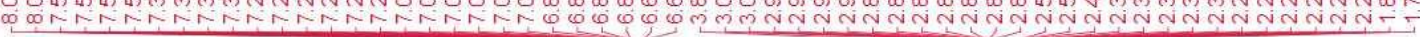

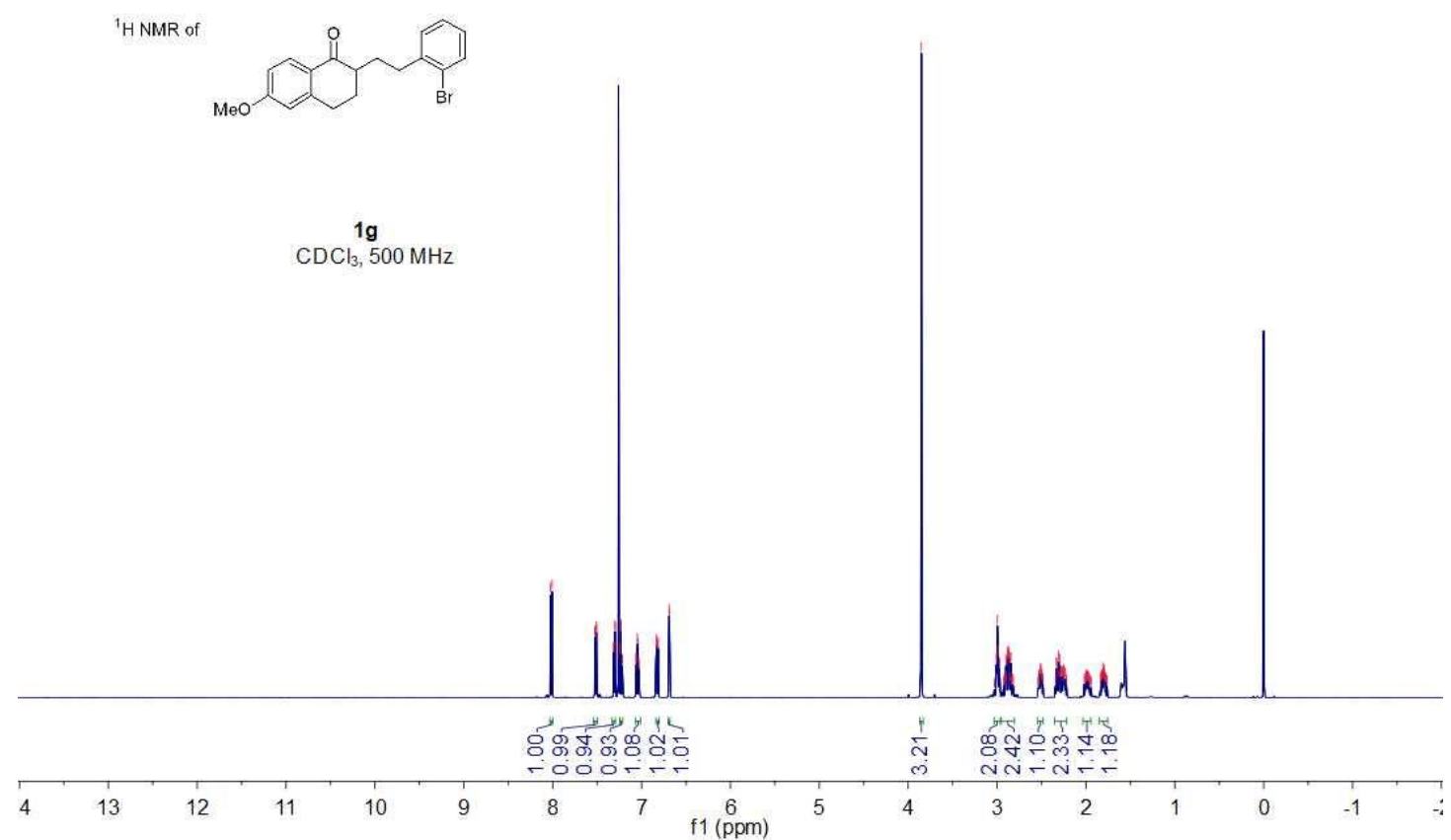




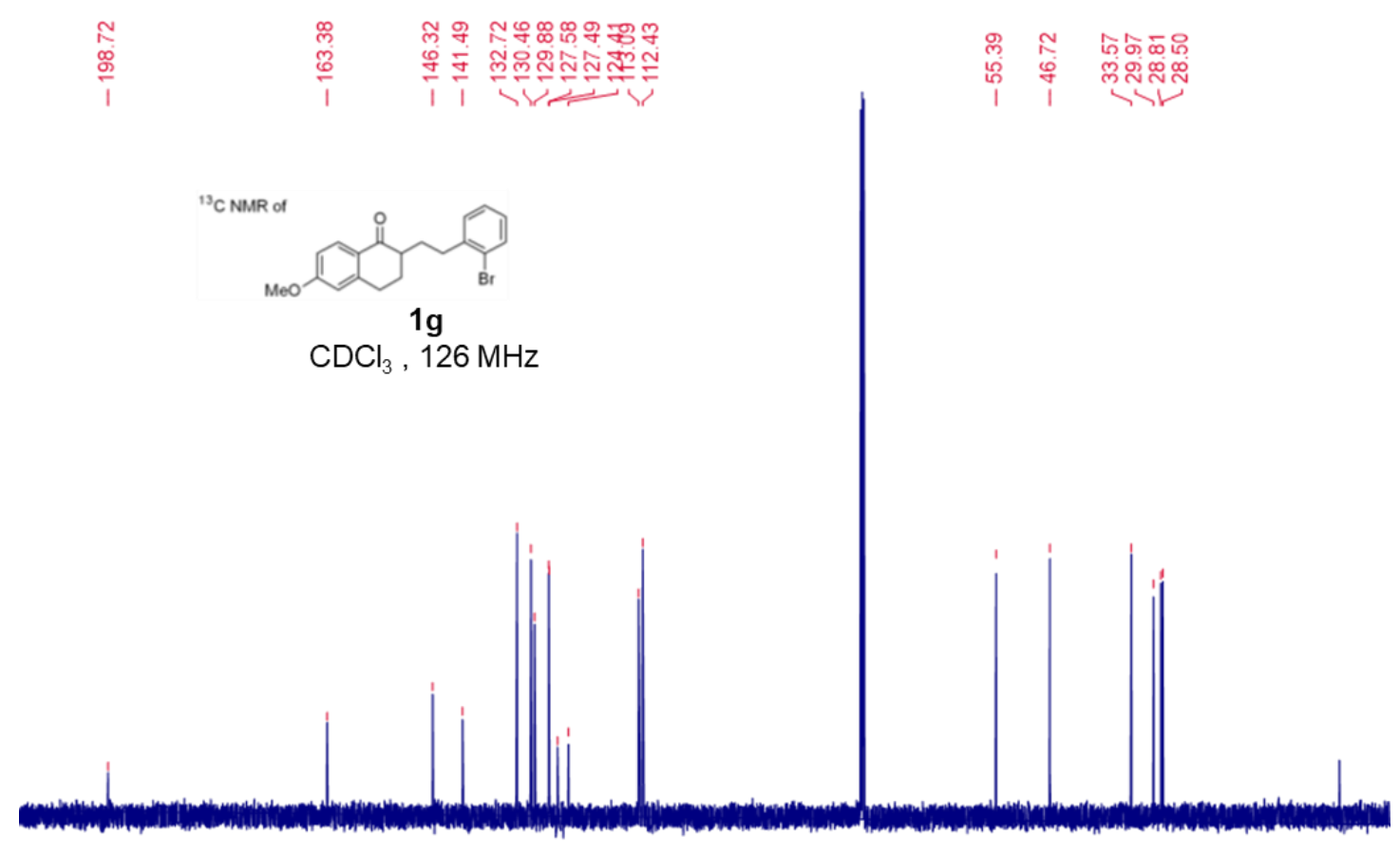

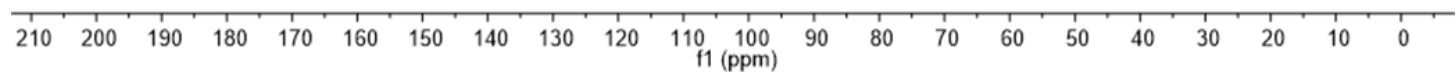

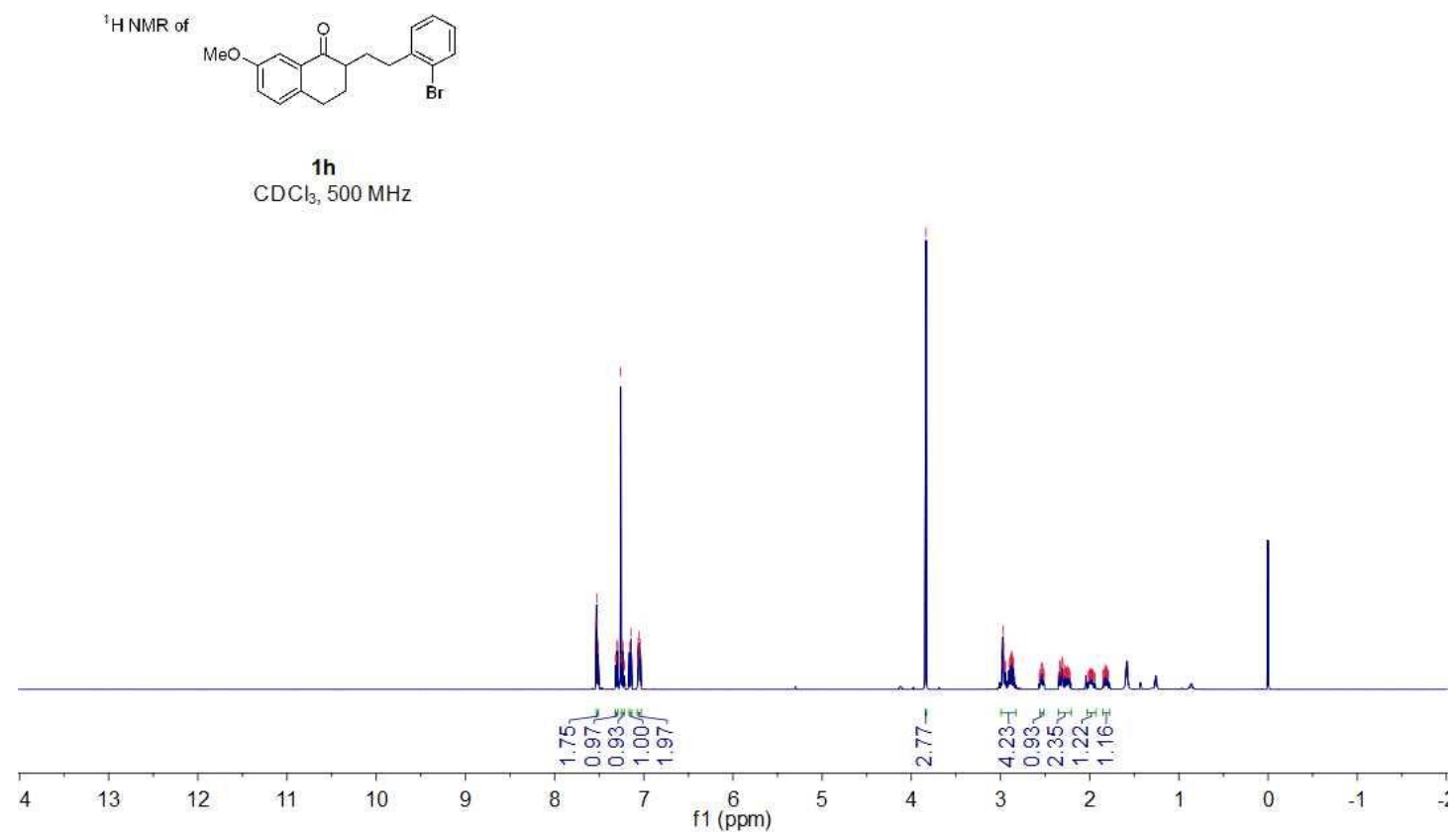



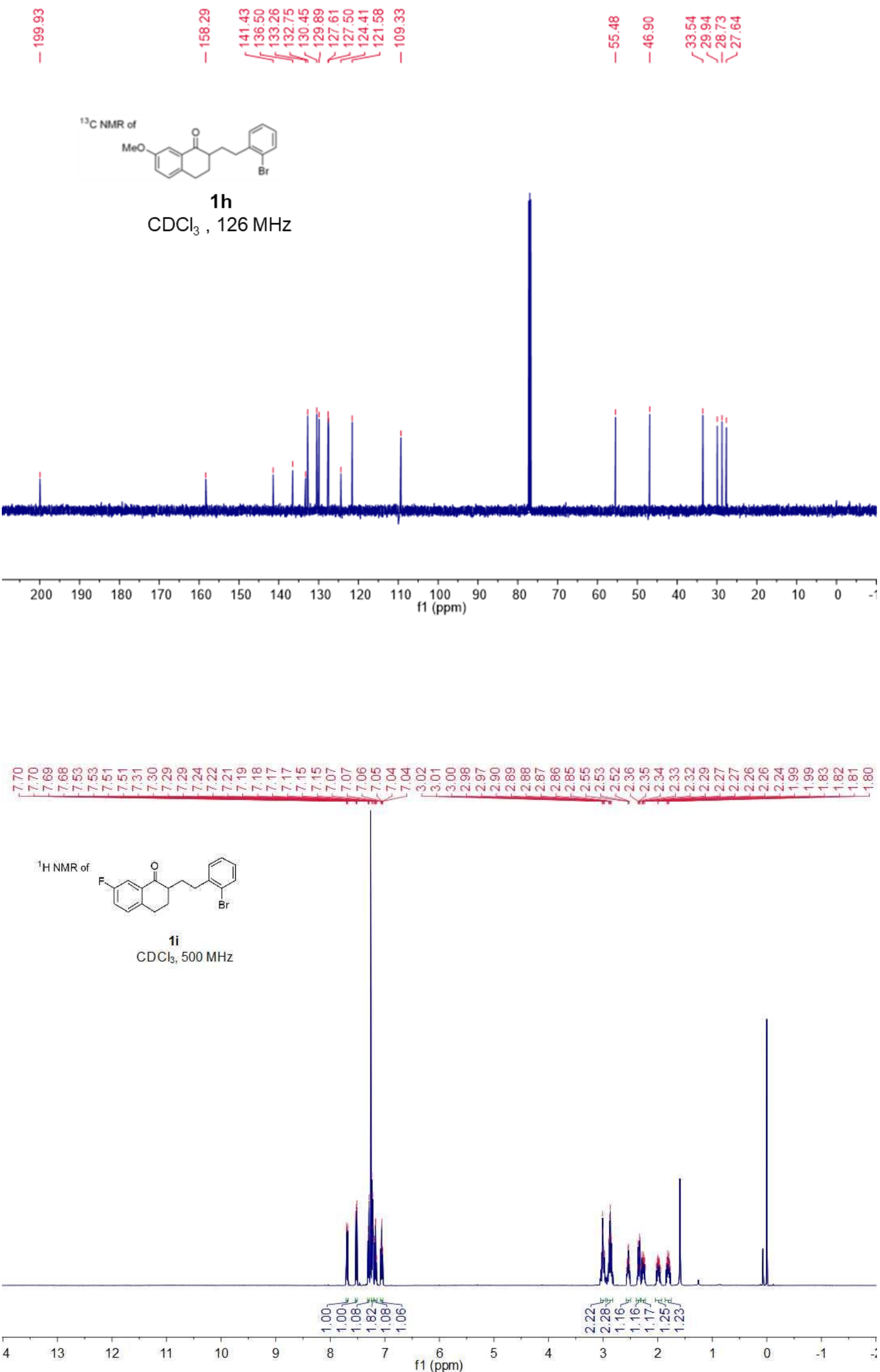


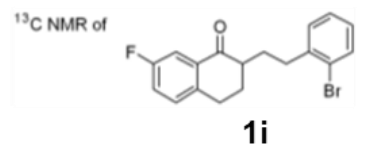

$\mathrm{CDCl}_{3}, 126 \mathrm{MHz}$

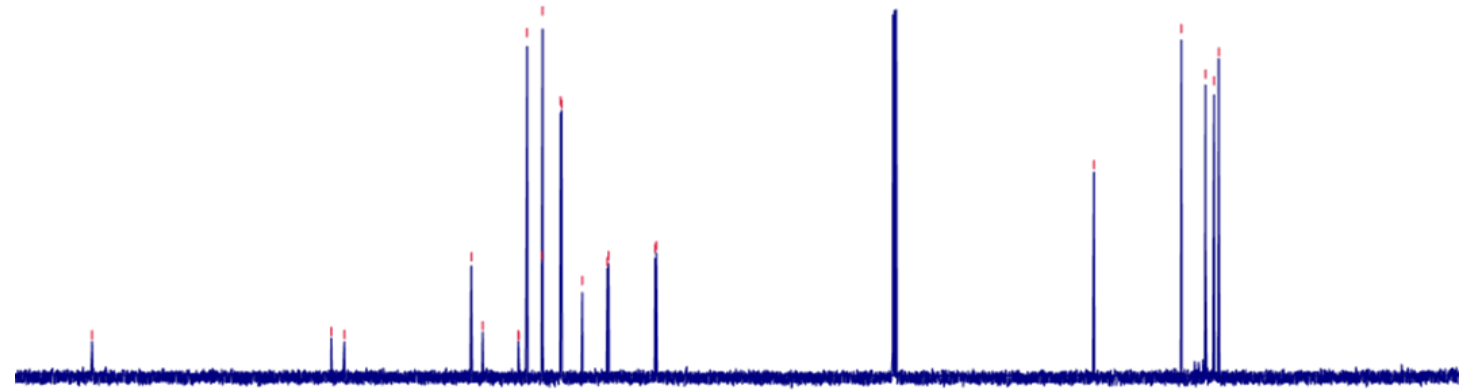

$\begin{array}{lllllllllllllllllllllllllllll}10 & 200 & 190 & 180 & 170 & 160 & 150 & 140 & 130 & 120 & 110 & 100 & 90 & 80 & 70 & 60 & 50 & 40 & 30 & 20 & 10 & 0\end{array}$

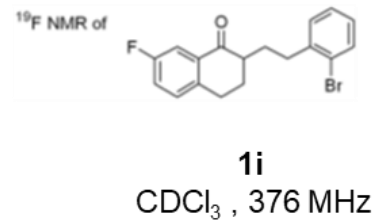

$\begin{array}{lllllllllllll}30 & 20 & 10 & 0 & -10 & -20 & -30 & -40 & -50 & -60 & -70 & -80 & -90\end{array}$ 
${ }^{1}$ HNMR of

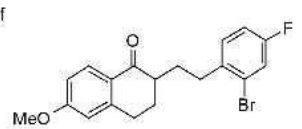

$\stackrel{\mathbf{1 j}}{\mathrm{CDCl}_{3}, 500 \mathrm{MHz}}$

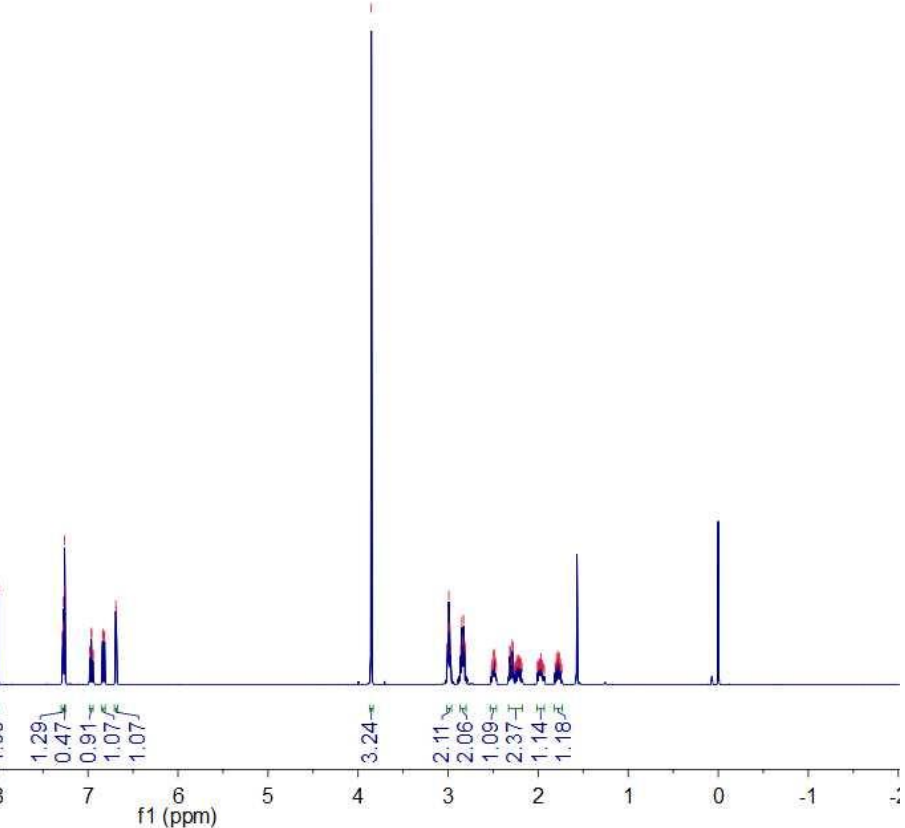

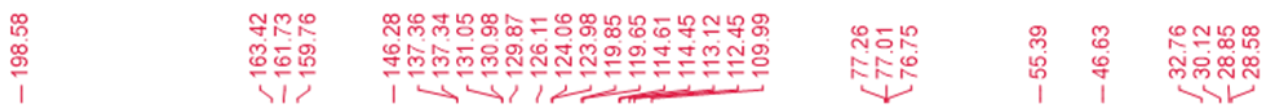

${ }^{13} \mathrm{C}$ NMR of
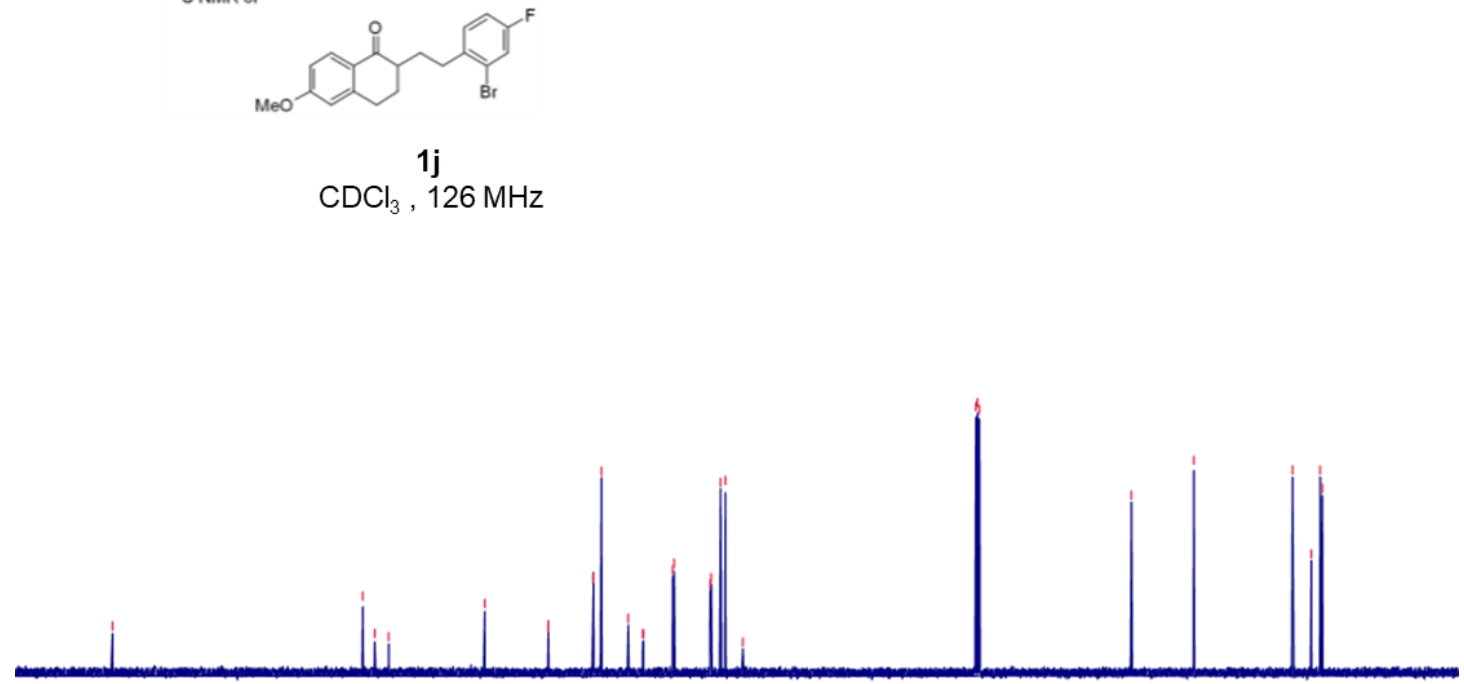

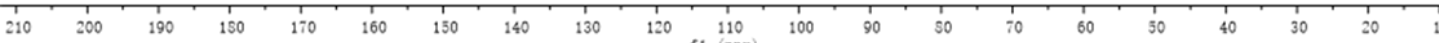



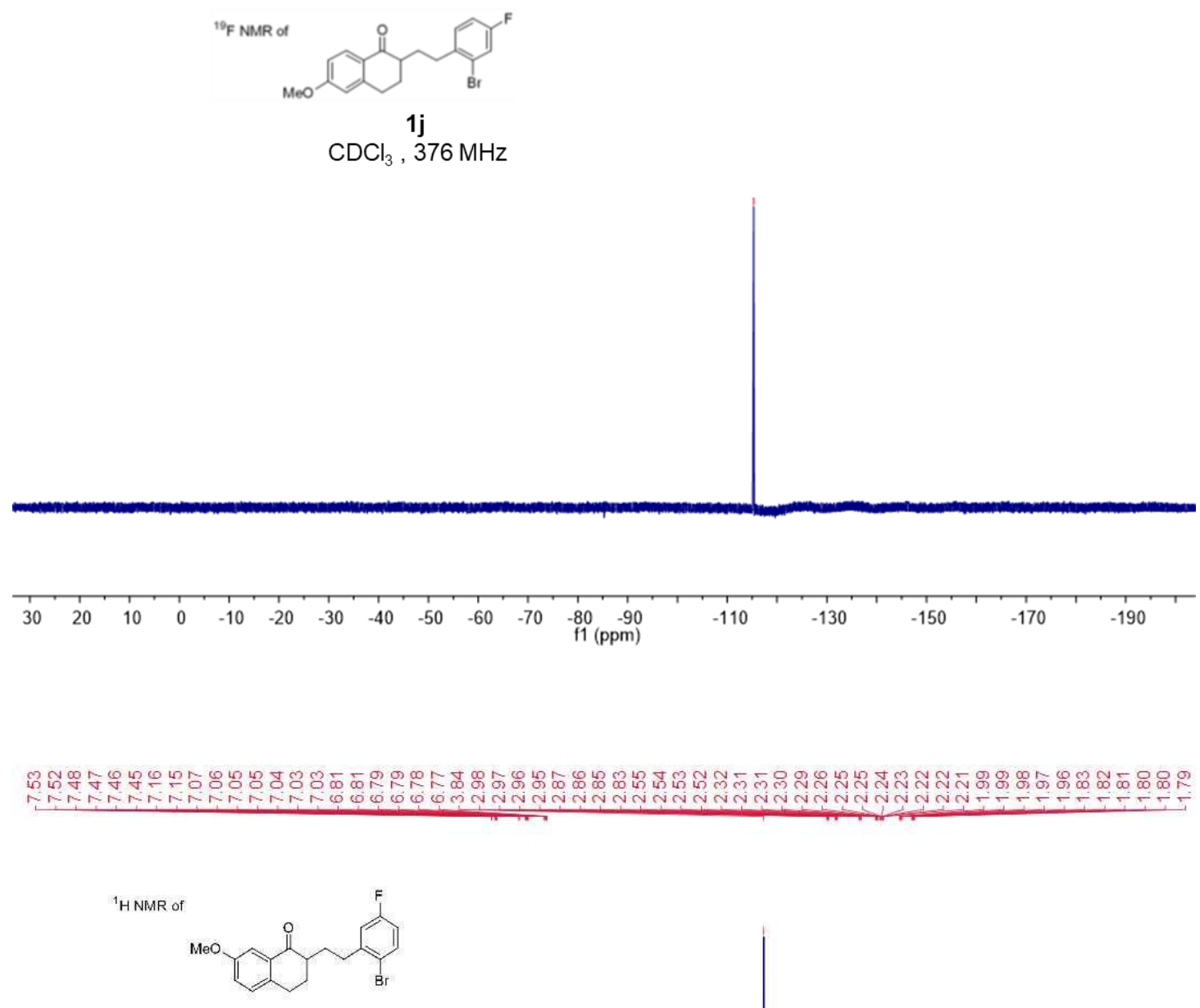

$$
\stackrel{1 \mathbf{k}}{\mathrm{CDCl}_{3}, 500 \mathrm{MHz}}
$$

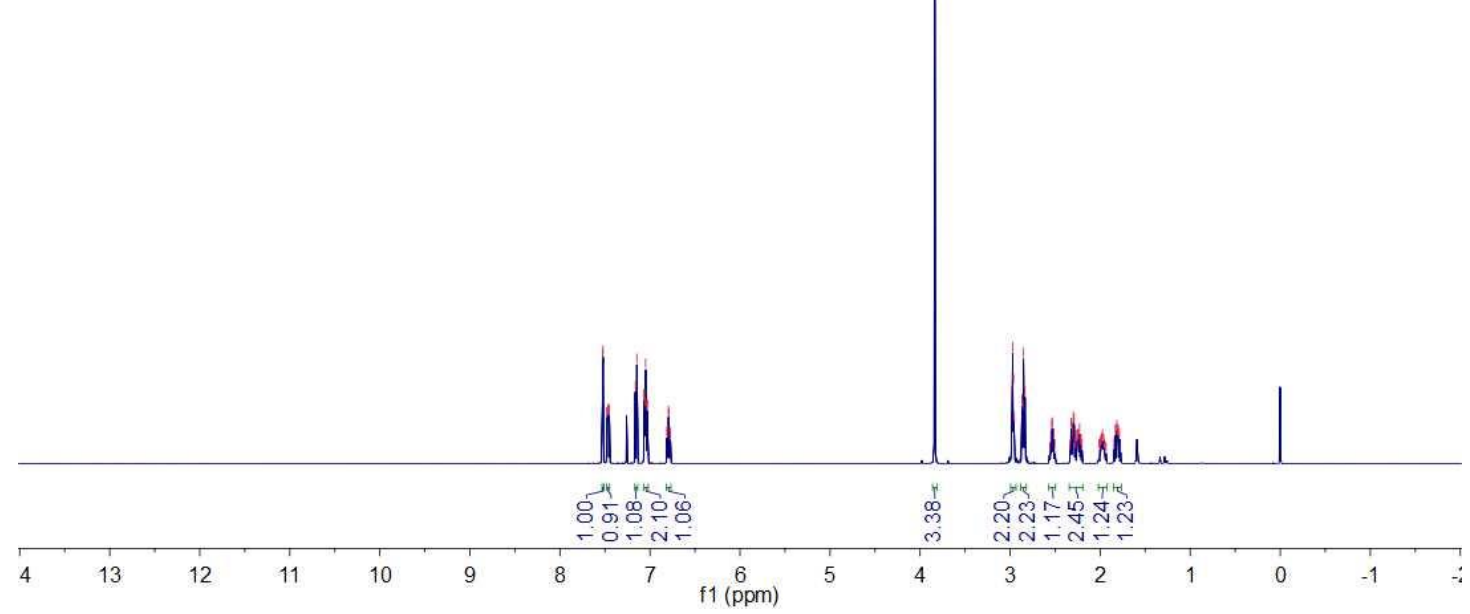




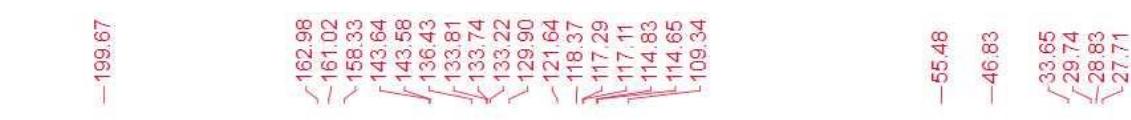

${ }^{13} \mathrm{C}$ NMR of<smiles>COc1ccc2c(c1)C(=O)C(CCc1cc(F)ccc1Br)CC2</smiles>

$1 k$

$\mathrm{CDCl}_{3}, 126 \mathrm{MHz}$
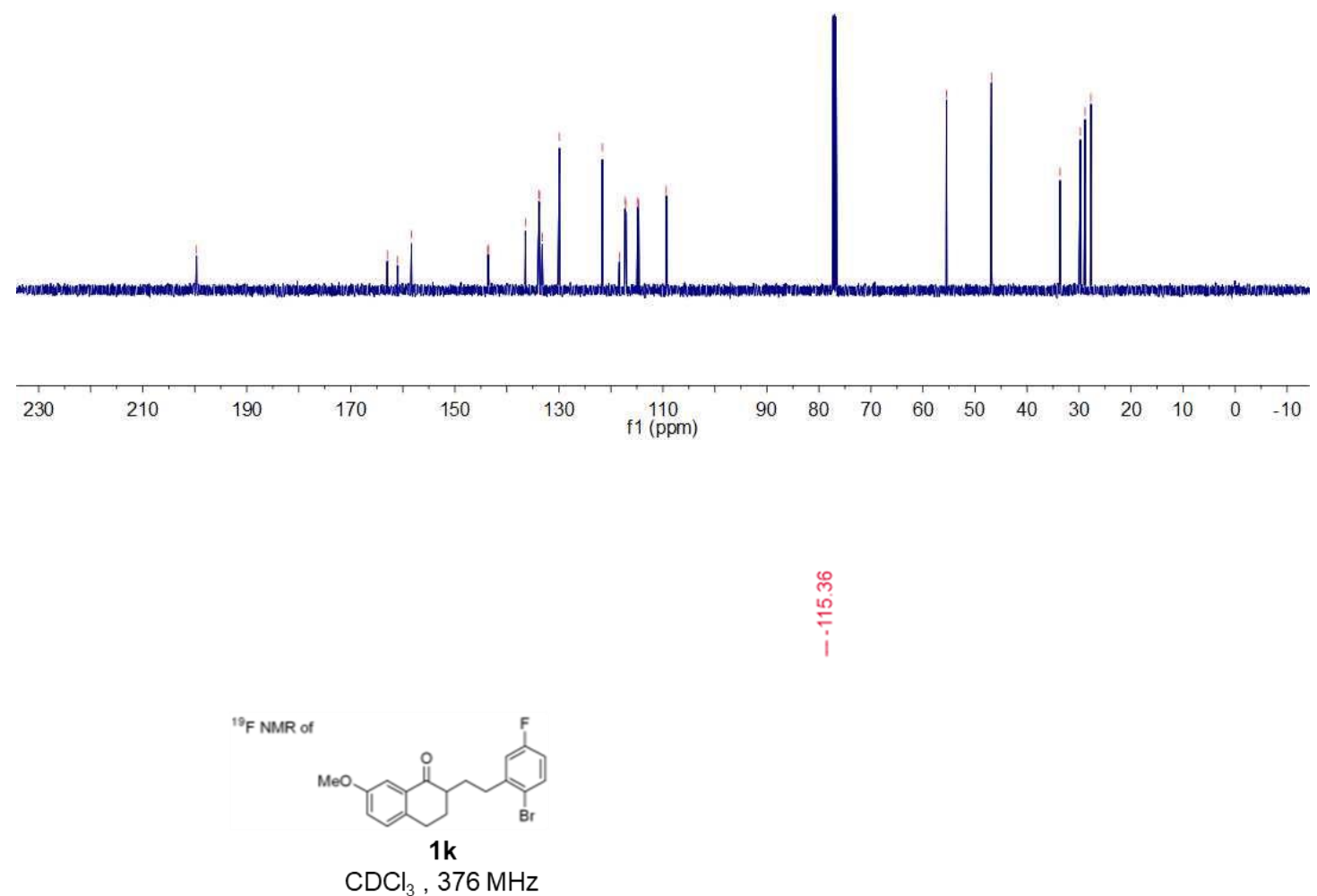

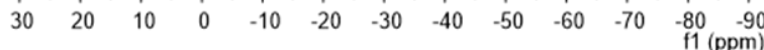



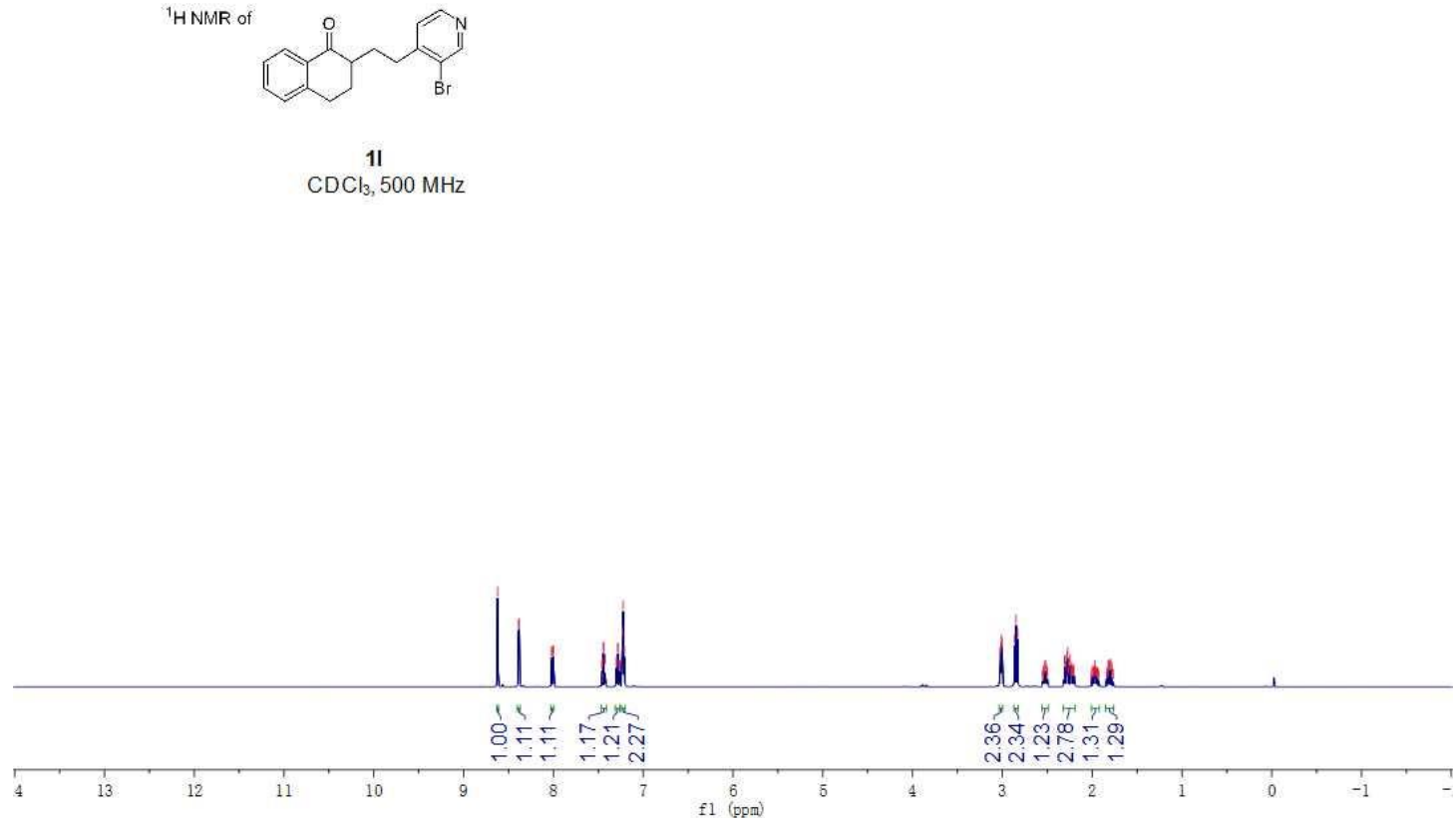

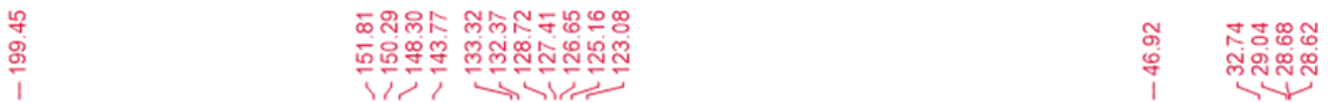

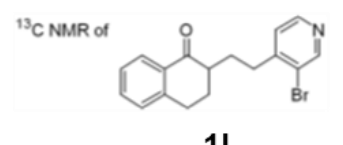

$\mathrm{CDCl}_{3}, 126 \mathrm{MHz}$
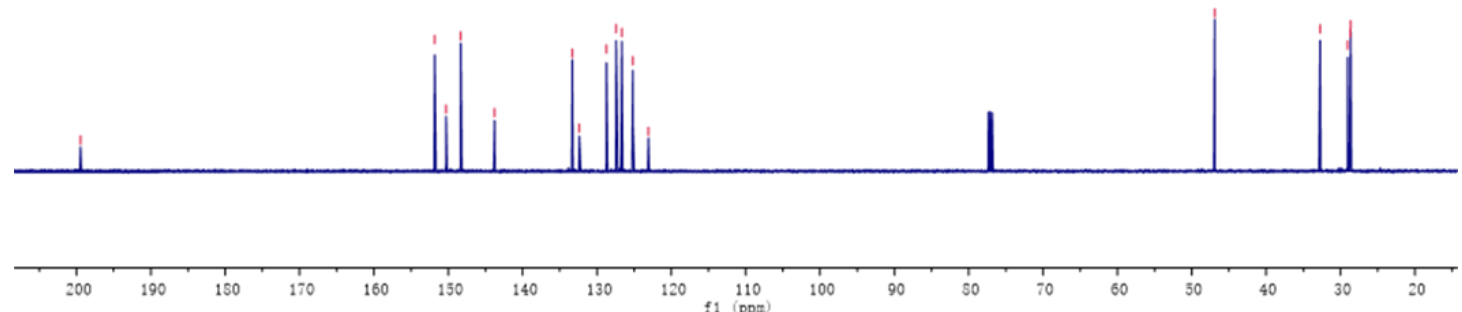


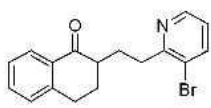

$\mathrm{CDCl}_{3}, 500 \mathrm{MHz}$

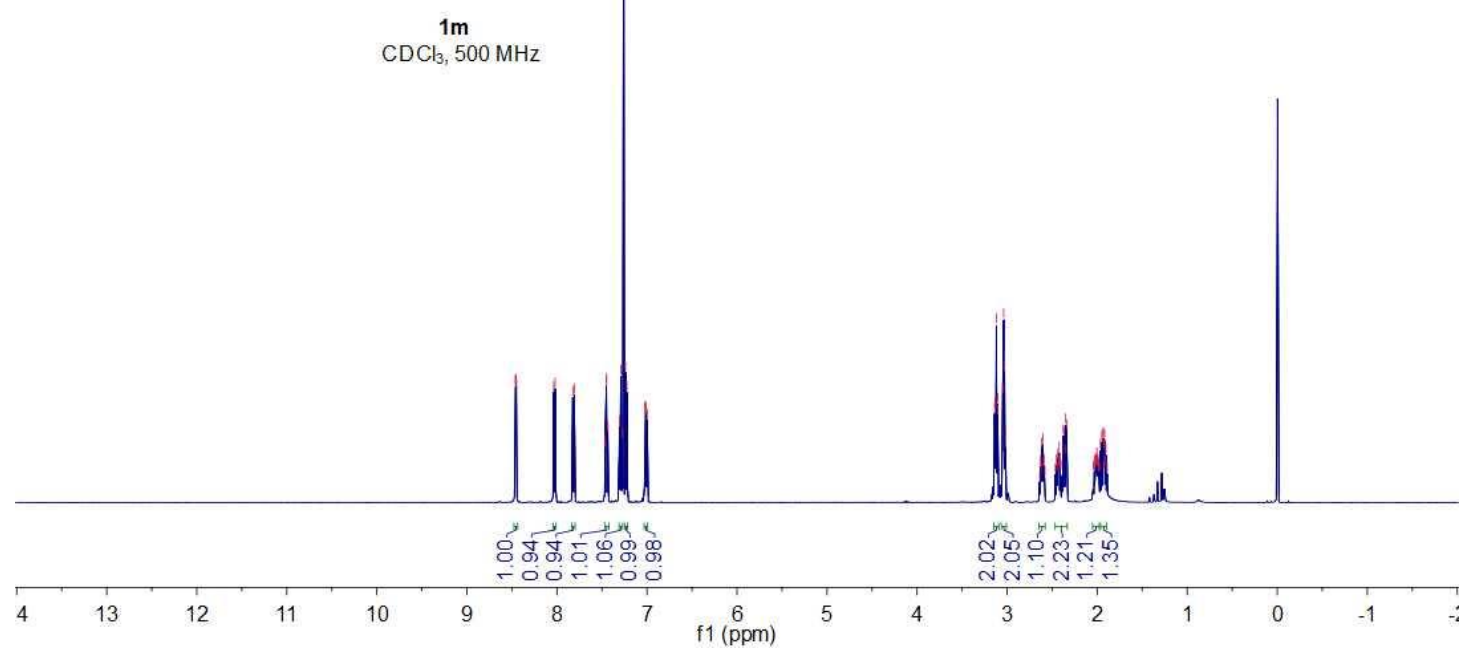

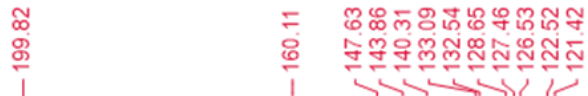

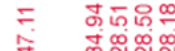

${ }^{13} \mathrm{C}$ NMR of

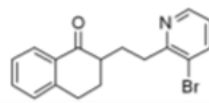

$1 \mathrm{~m}$

$\mathrm{CDCl}_{3}, 126 \mathrm{MHz}$

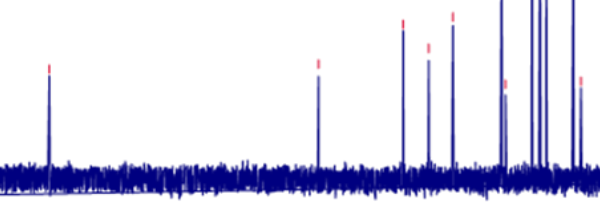

$\begin{array}{llllllllll}200 & 190 & 180 & 170 & 160 & 150 & 140 & 130 & 120 & 110 \begin{array}{l}100 \\ \mathrm{f} 1(\mathrm{ppm})\end{array}\end{array}$ 
${ }^{1} \mathrm{H}$ NMR of

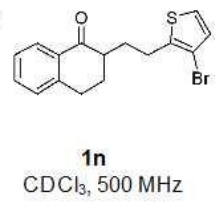

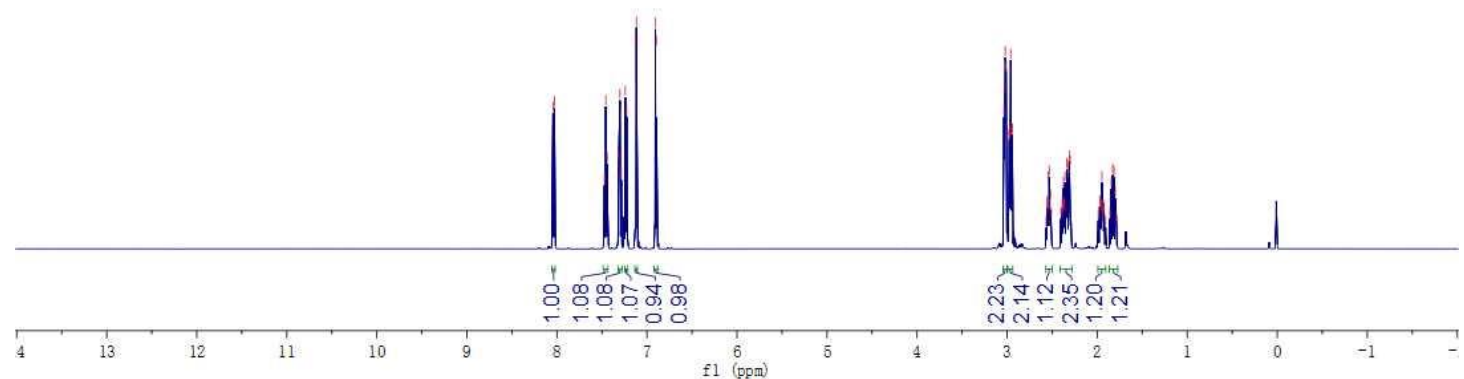
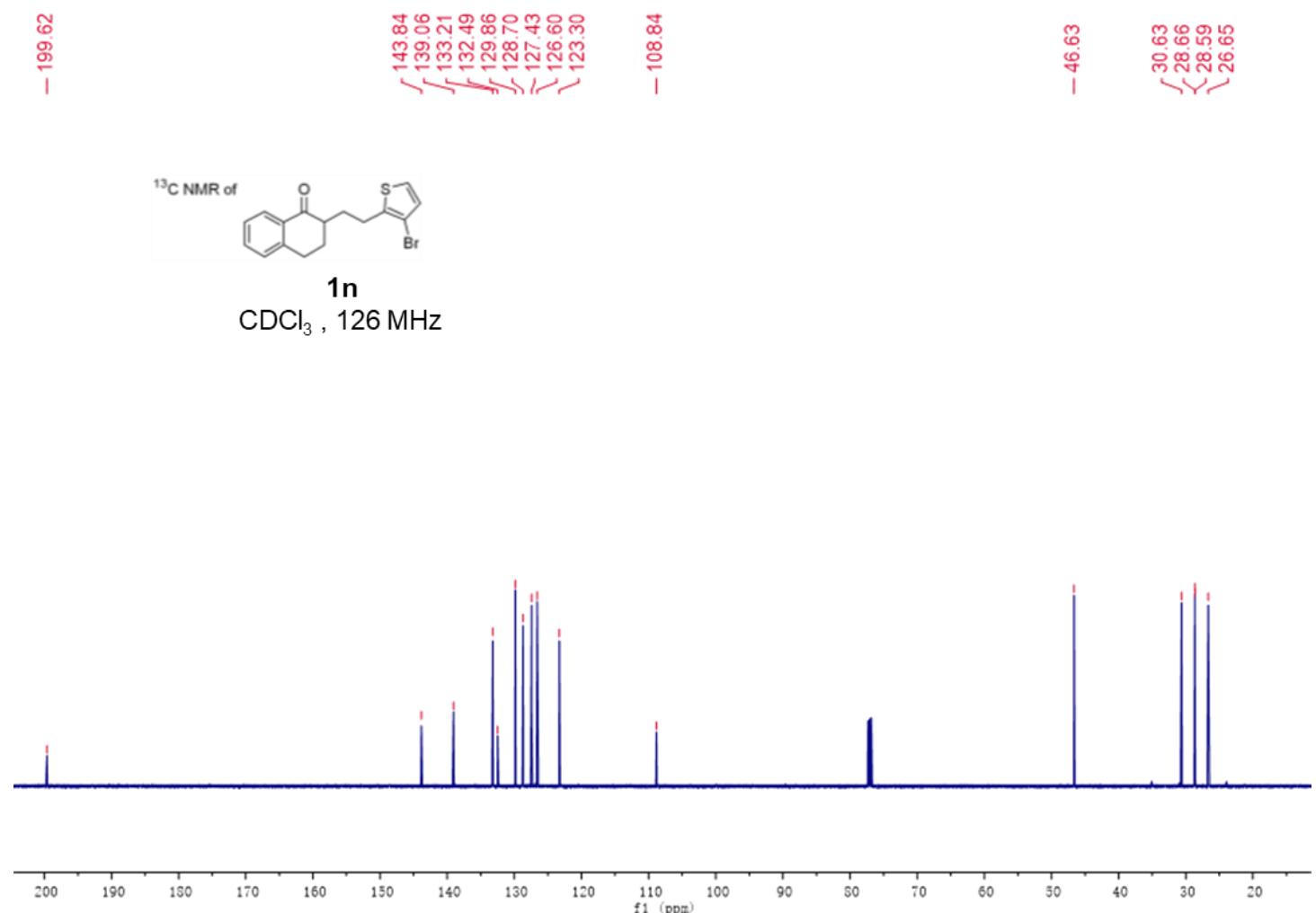
${ }^{1} \mathrm{HNMR}$ of

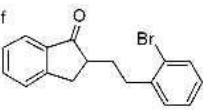

$\mathrm{CDCl}_{3}, 500 \mathrm{MHz}$
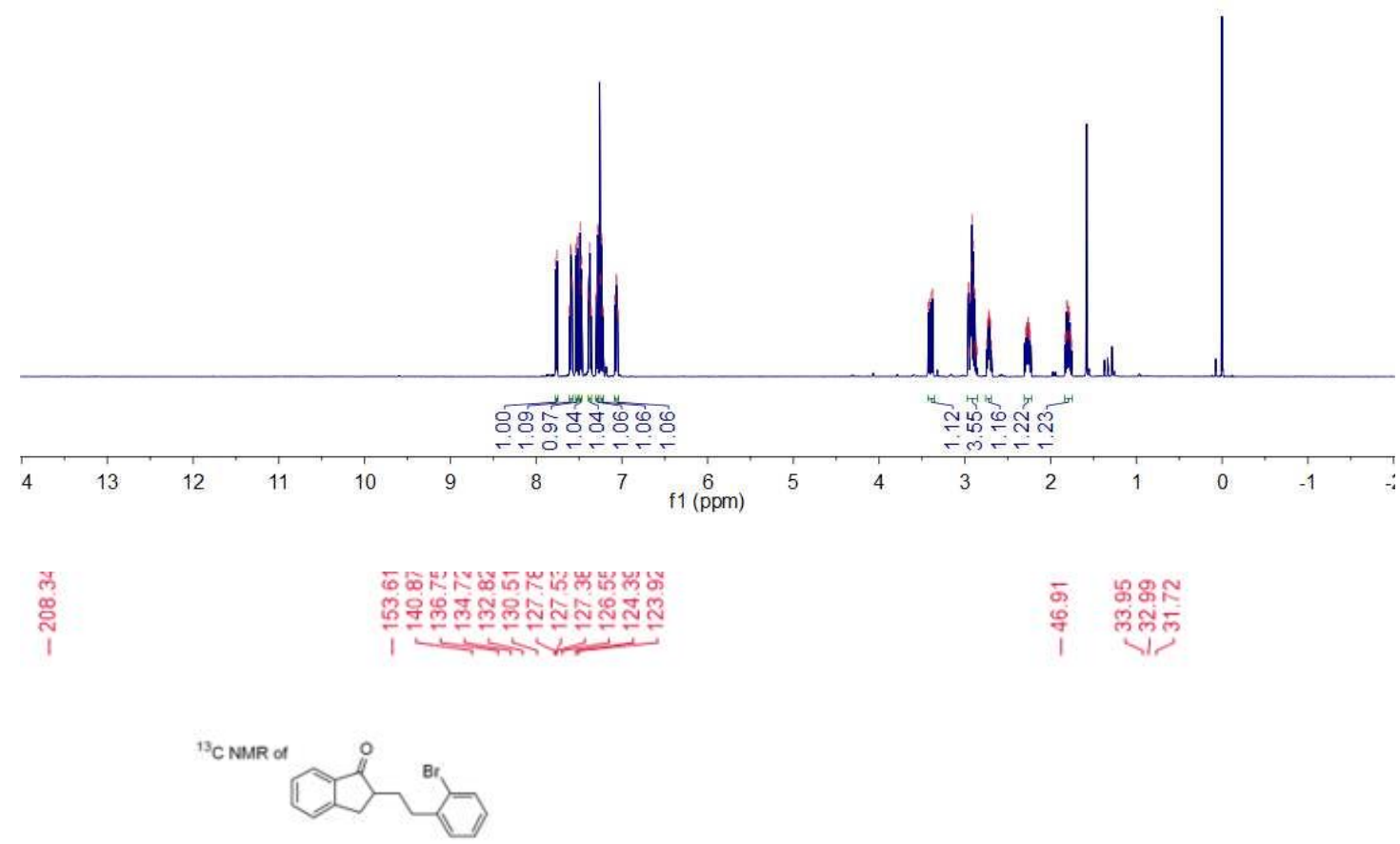

10

$\mathrm{CDCl}_{3}, 126 \mathrm{MHz}$

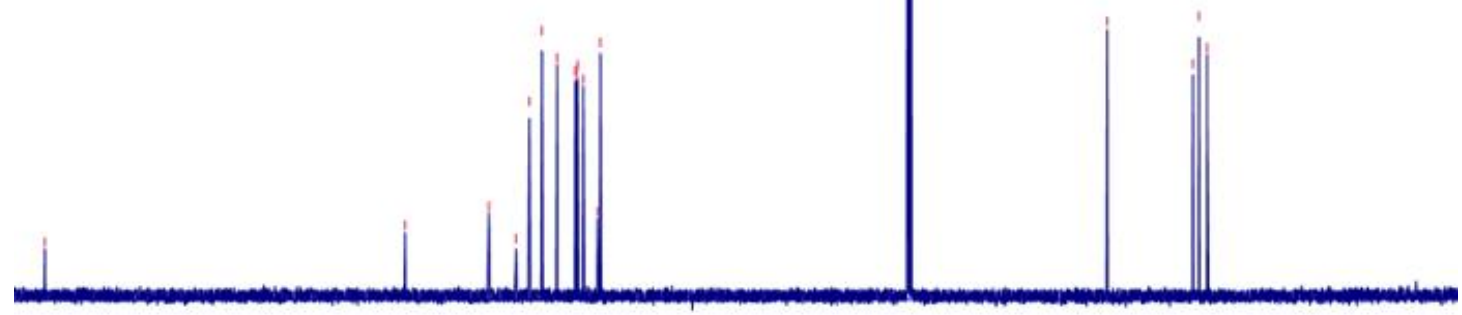

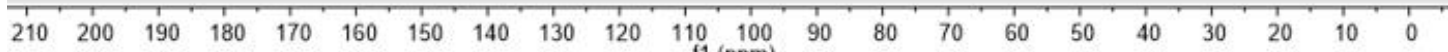



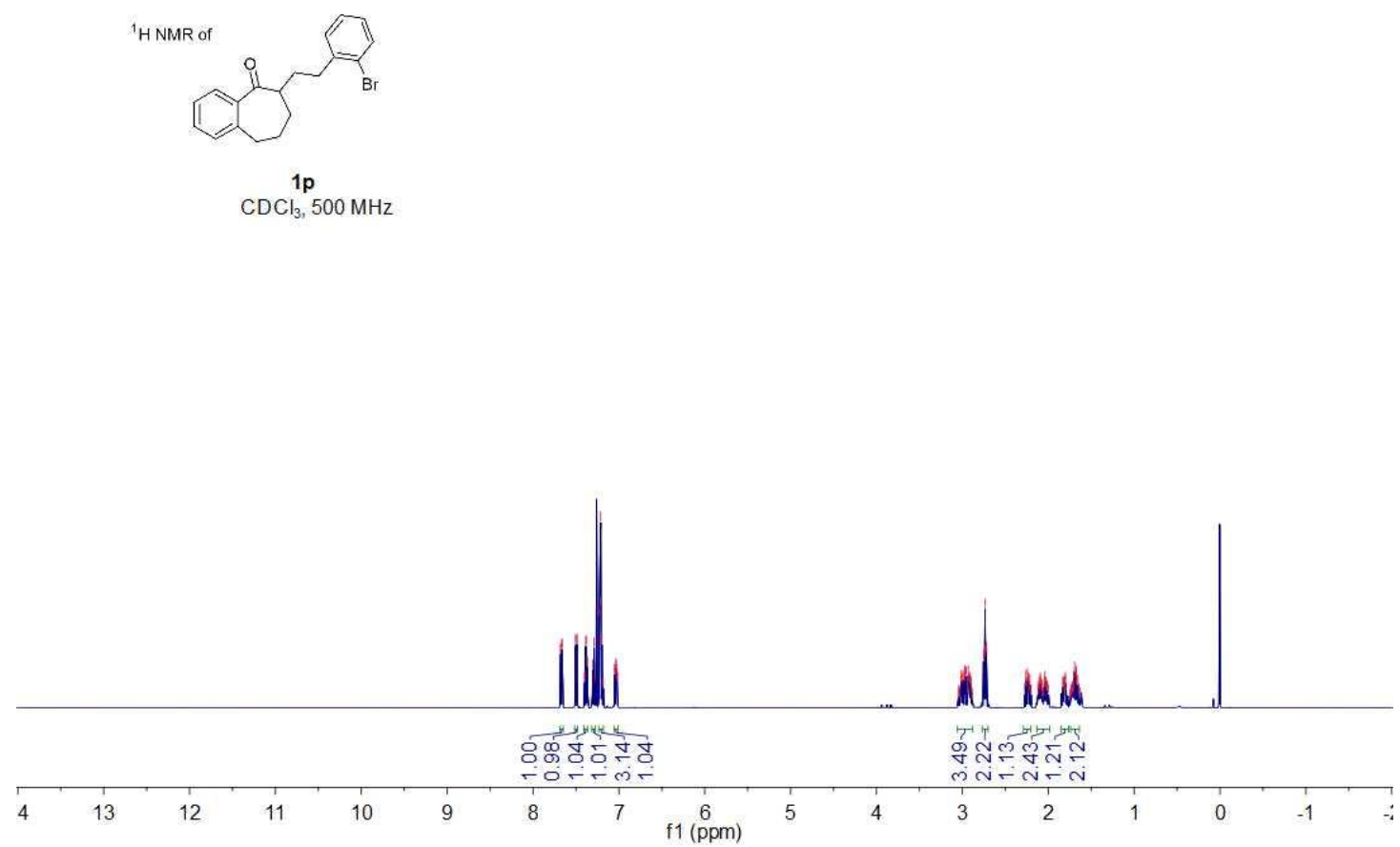

\begin{tabular}{|c|c|c|}
\hline $\begin{array}{l}\text { ป } \\
\text { ஸे } \\
\text { Nิ }\end{array}$ & 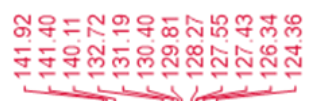 & $\begin{array}{l}\text { of } \\
\text { g }\end{array}$ \\
\hline
\end{tabular}

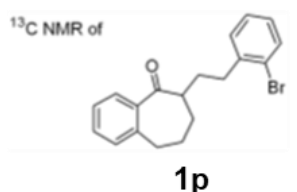

$\mathrm{CDCl}_{3}, 126 \mathrm{MHz}$

$\begin{array}{llllllllllll}210 & 200 & 190 & 180 & 170 & 160 & 150 & 140 & 130 & 120 & 110 & 100 \\ 11 & & & \end{array}$ 

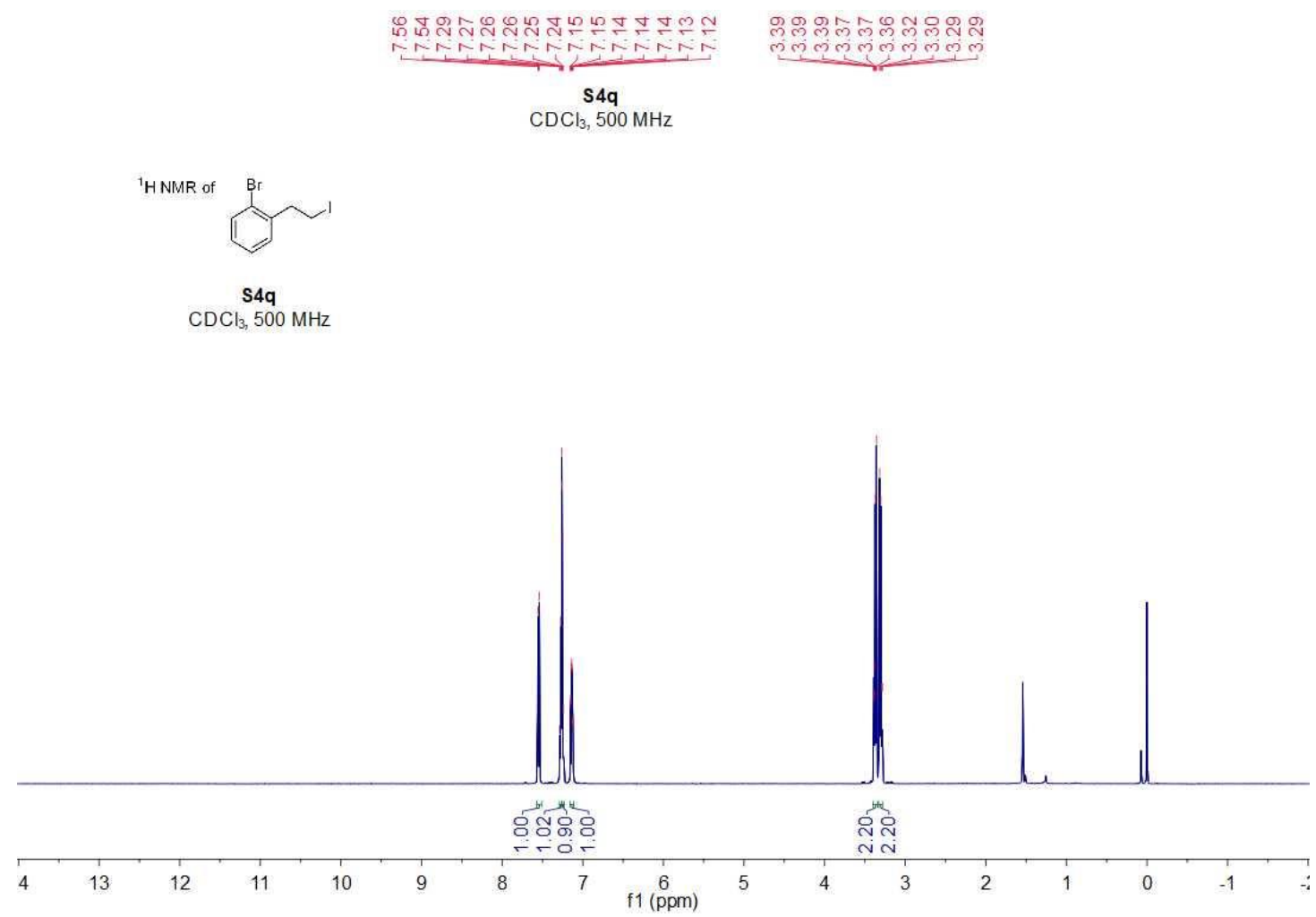

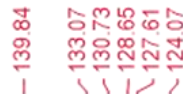
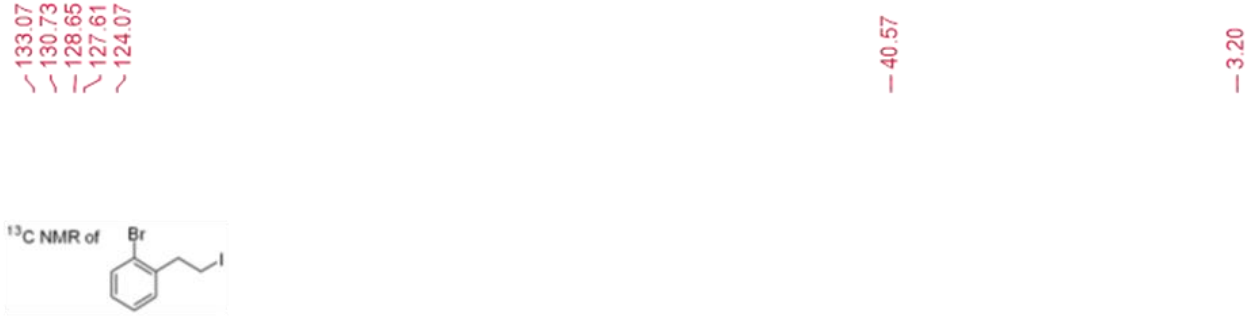

S4q

$\mathrm{CDCl}_{3}, 126 \mathrm{MHz}$
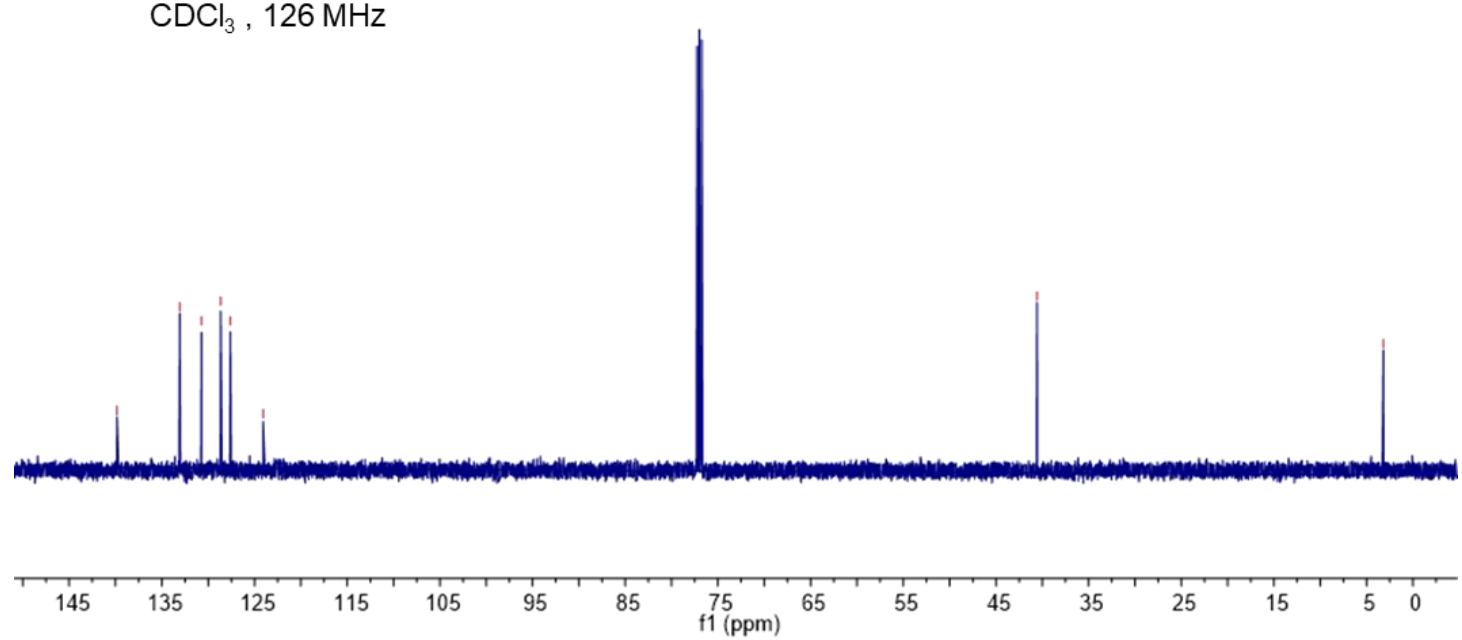
'HNMR of

S4r

$\mathrm{CDCl}_{3}, 500 \mathrm{MHz}$

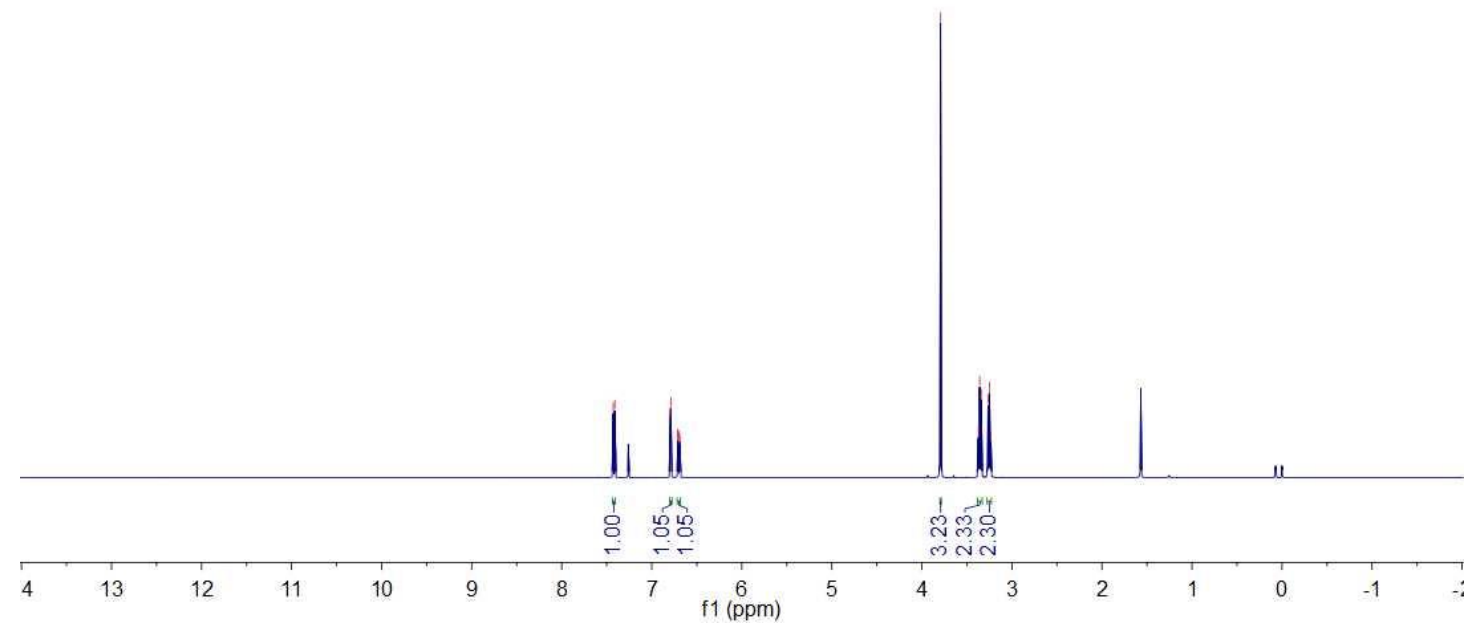

$\frac{1}{\infty}$ 年

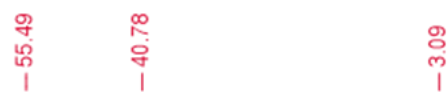

${ }^{13} \mathrm{CNMR}$ of

S4r

$\mathrm{CDCl}_{3}, 126 \mathrm{MHz}$

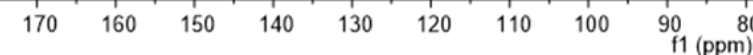




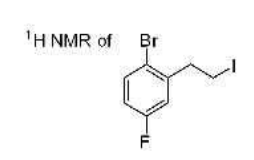

s4s

$\mathrm{CDCl}_{3}, 500 \mathrm{MHz}$
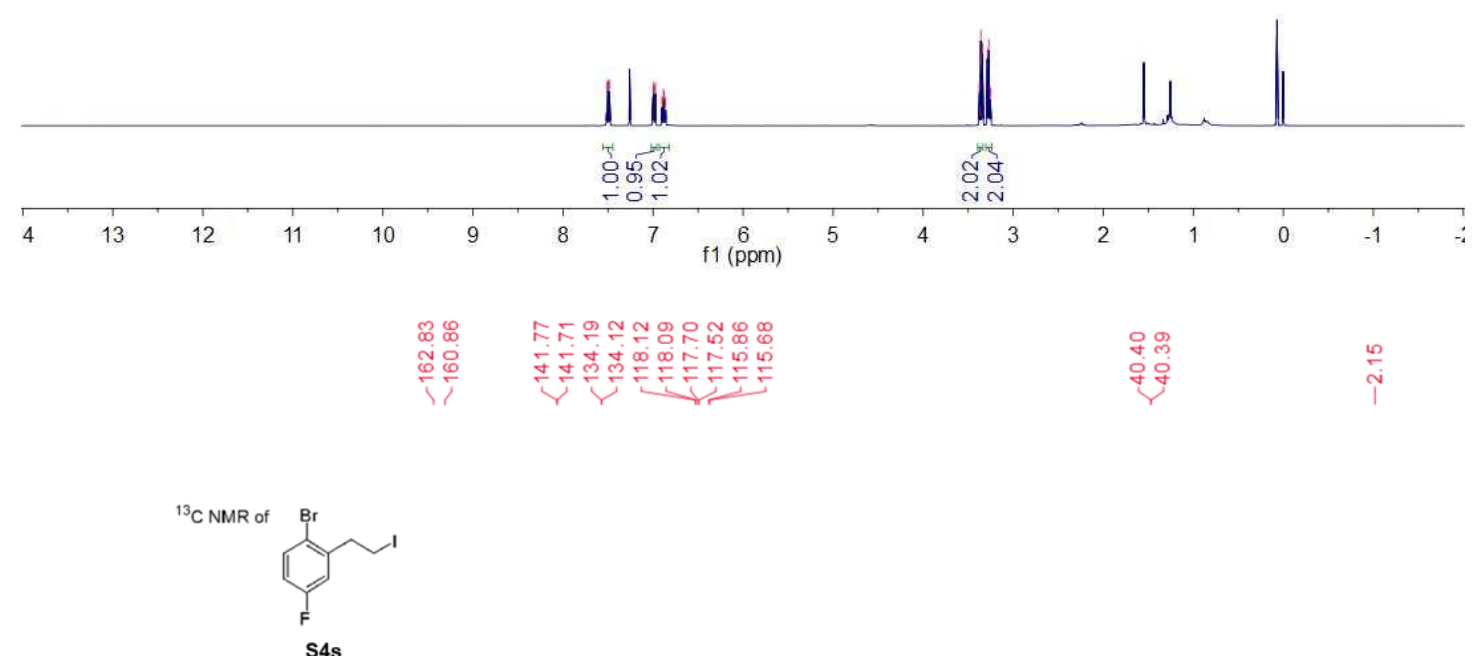

$\mathrm{CDCl}_{3}, 126 \mathrm{MHz}$

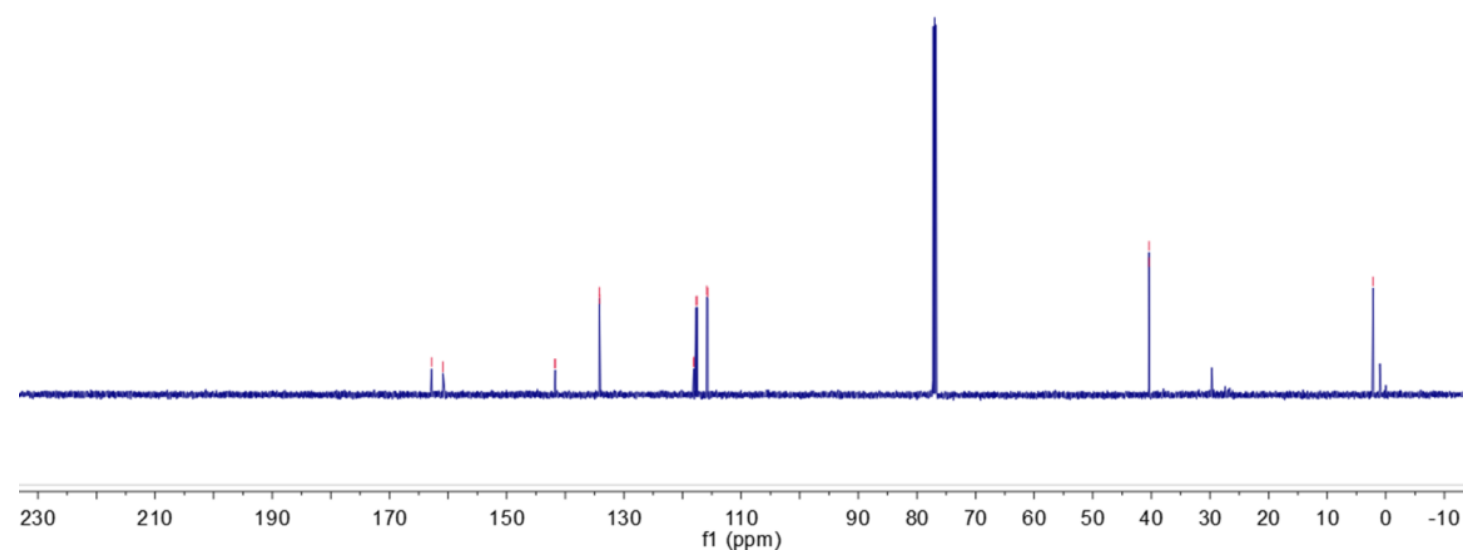




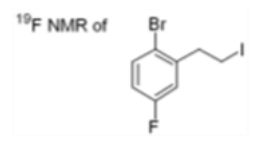

S4s

$\mathrm{CDCl}_{3}, 376 \mathrm{MHz}$
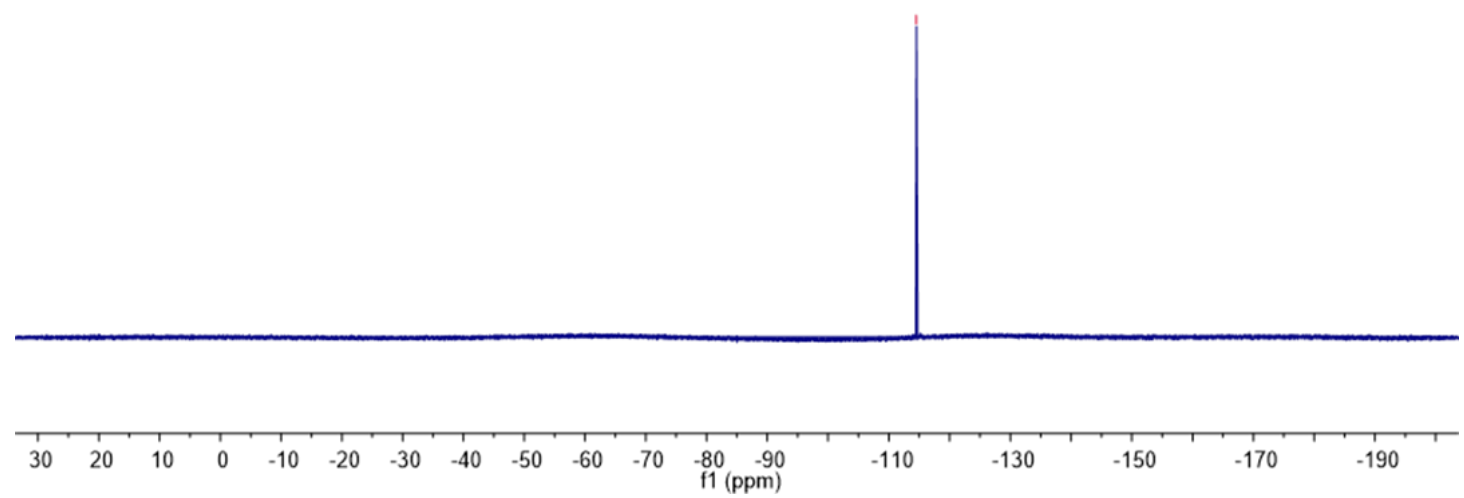

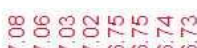

- NN

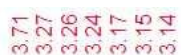

लिलाएँ

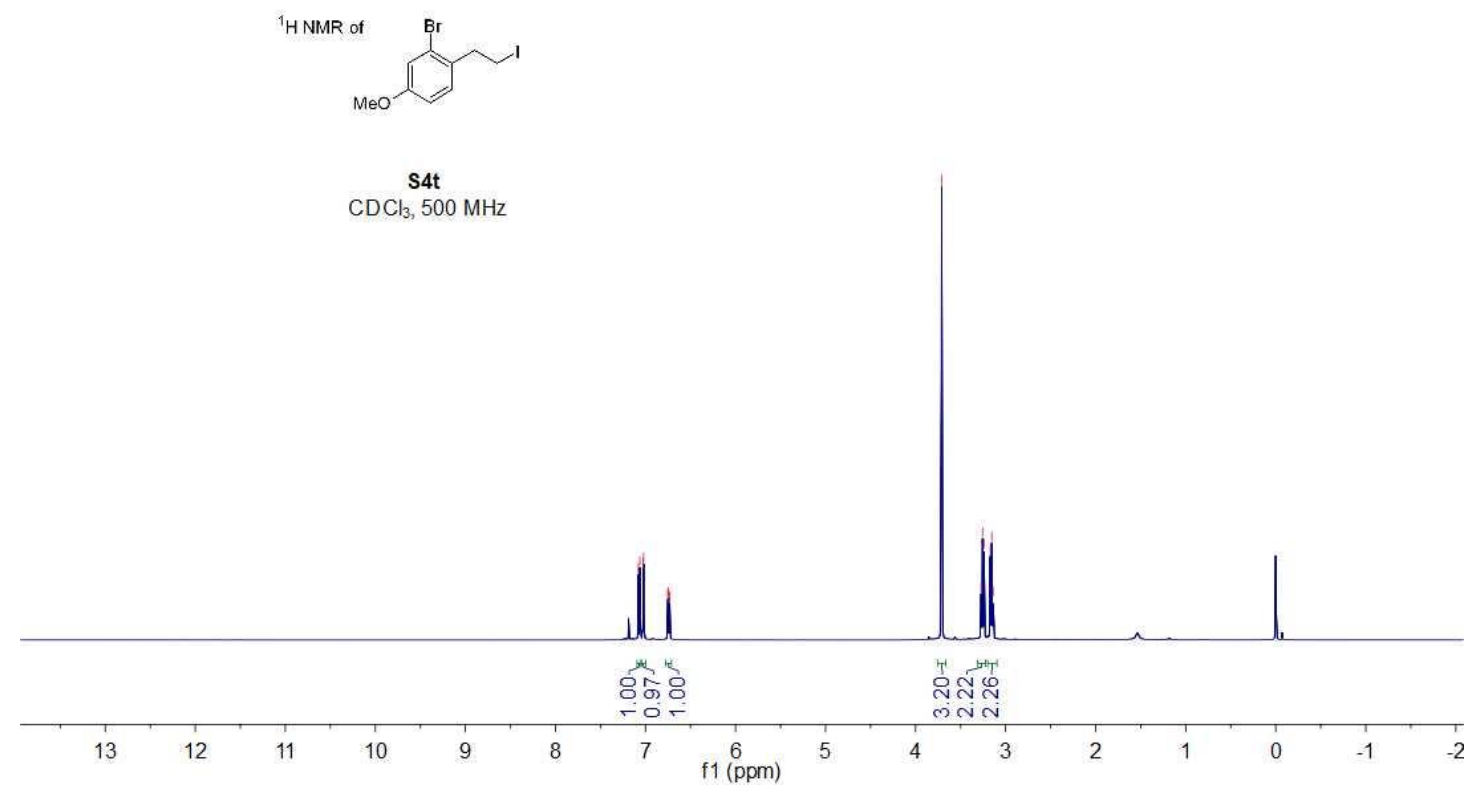




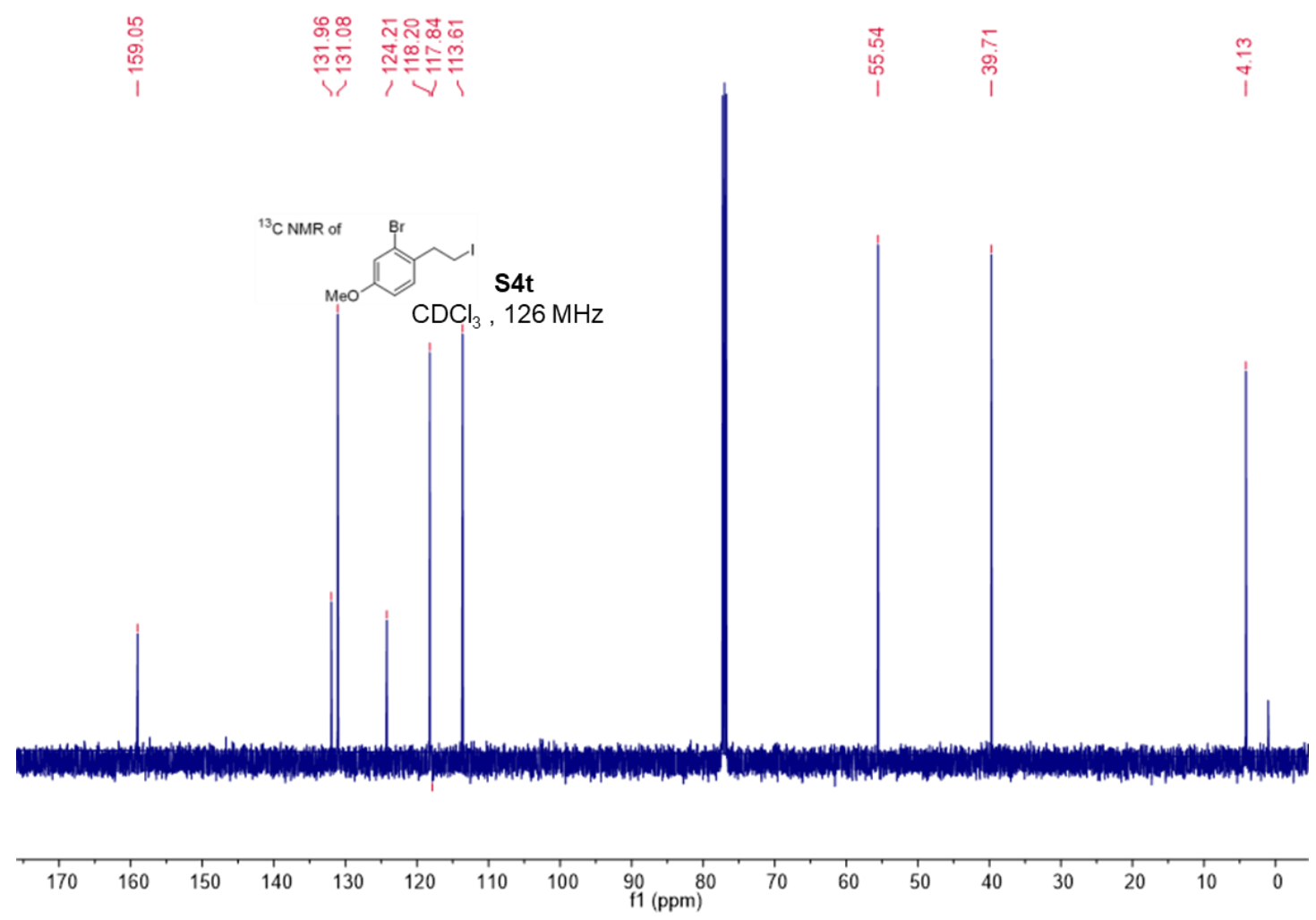

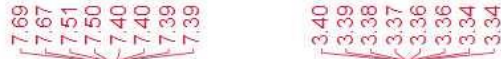

${ }^{1} \mathrm{H}$ NMR of

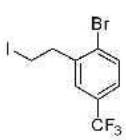

S4u

$\mathrm{CDCl}_{3}, 500 \mathrm{MHz}$

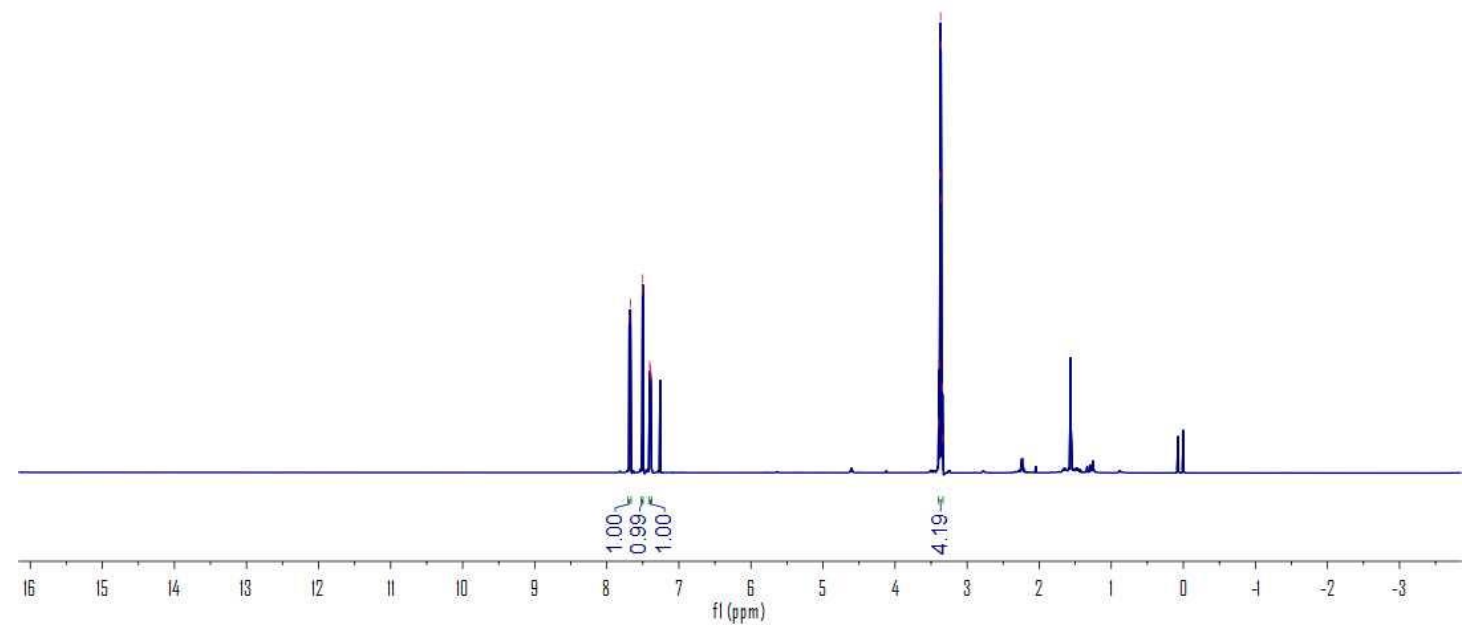




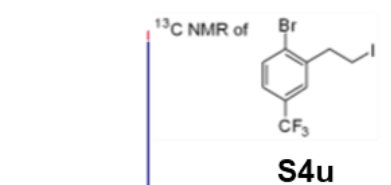

$\mathrm{CDCl}_{3}, 126 \mathrm{MHz}$
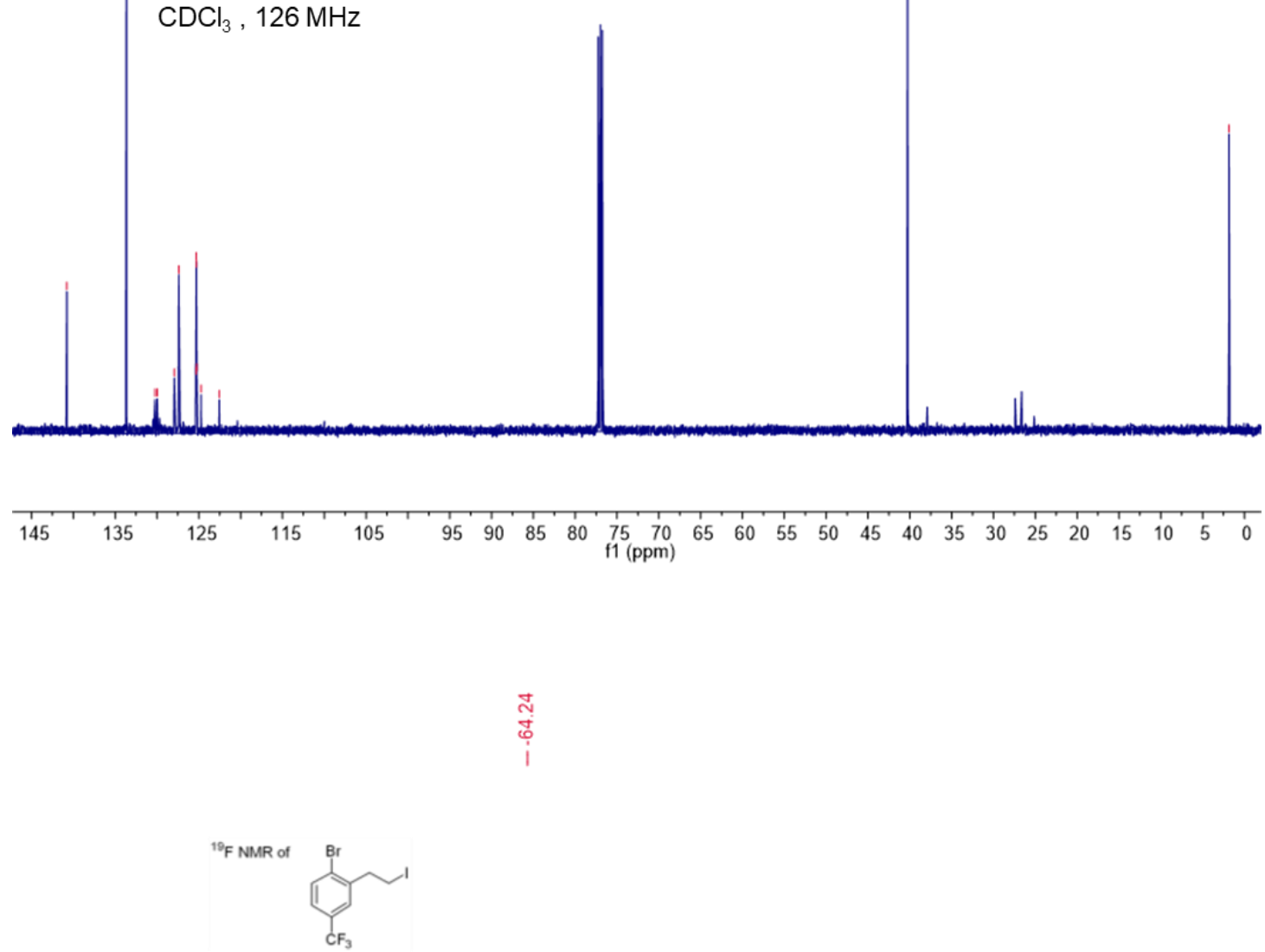

S4u

$\mathrm{CDCl}_{3}, 376 \mathrm{MHz}$

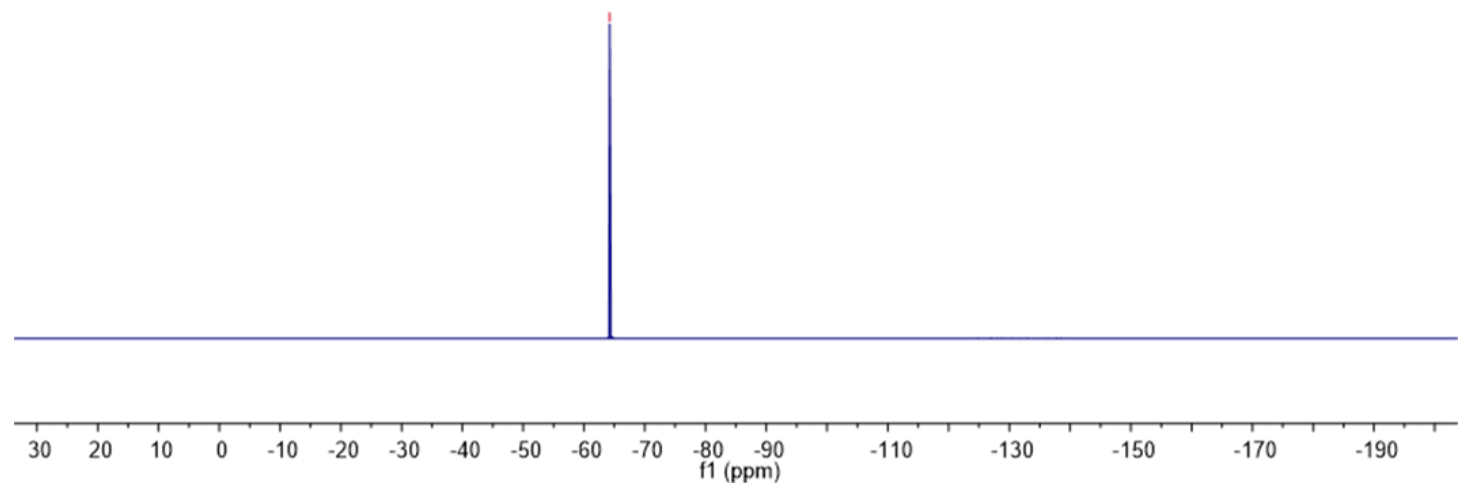




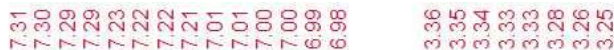
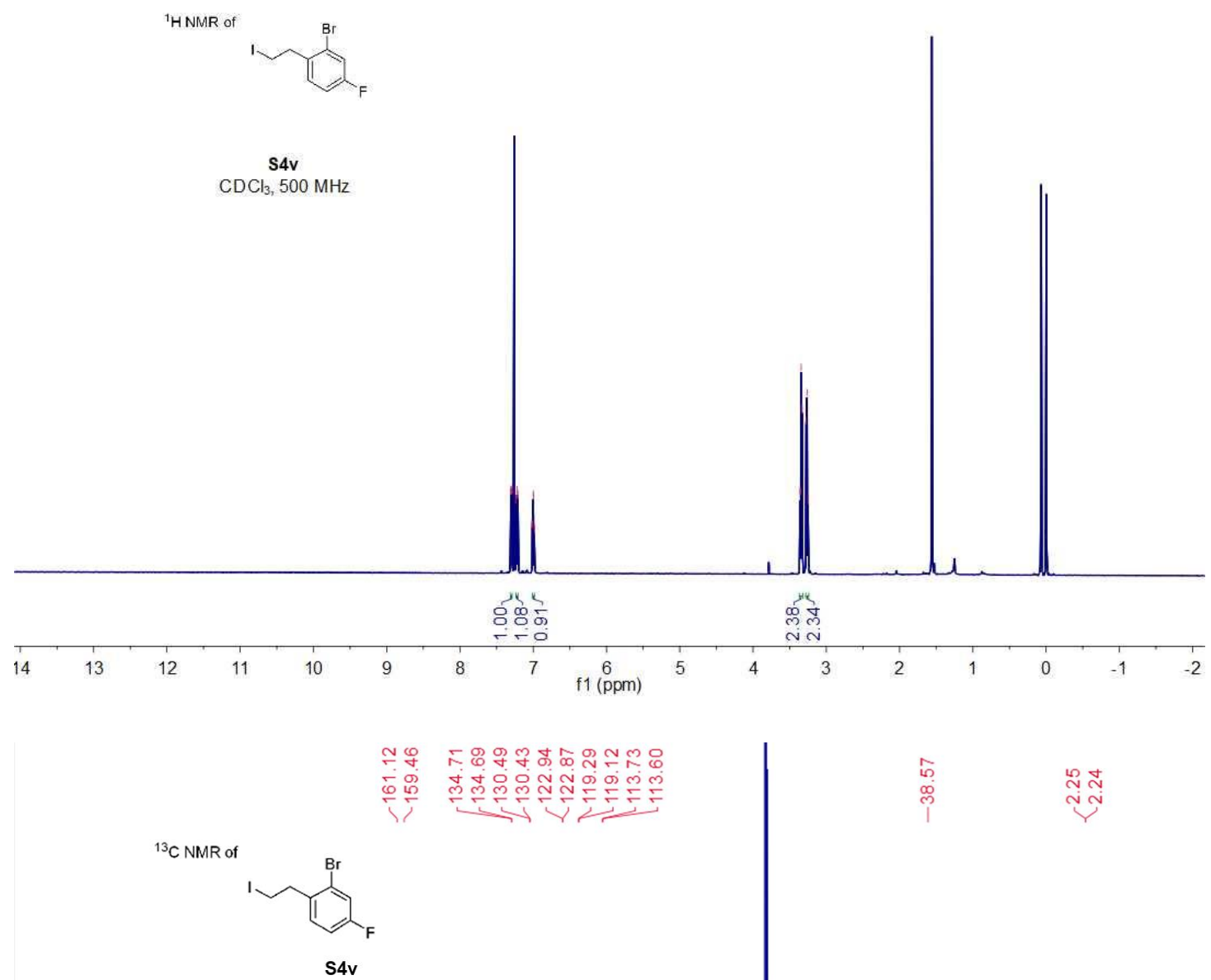

ㄴ.

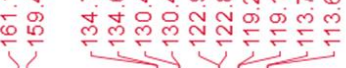

${ }^{13} \mathrm{C}$ NMR of
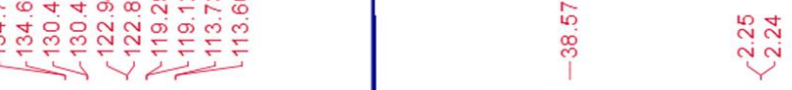

$\mathrm{CDCl}_{3}, 151 \mathrm{MHz}$

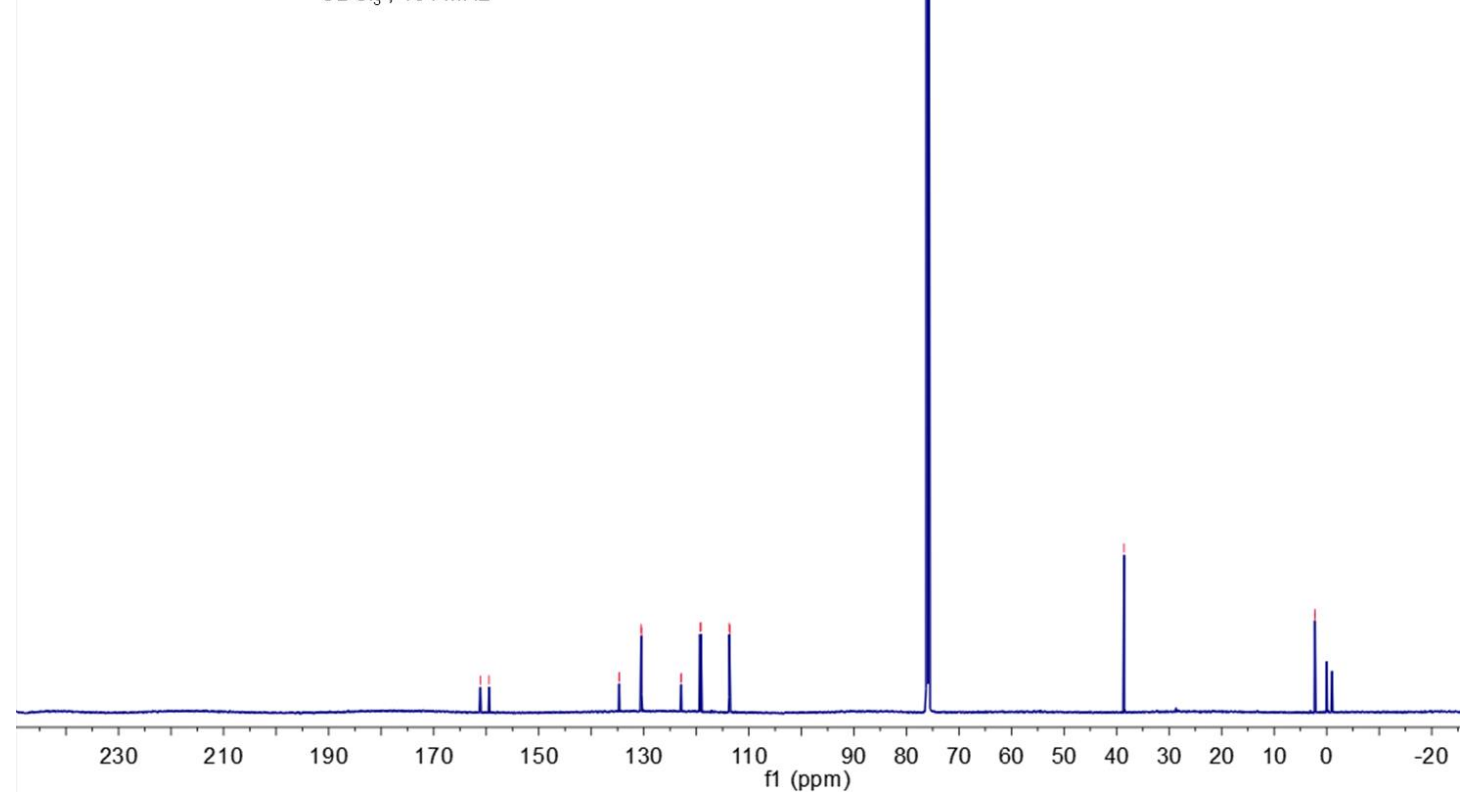




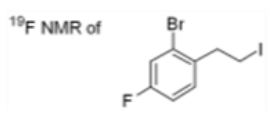

S4v

$\mathrm{CDCl}_{3}, 376 \mathrm{MHz}$

\begin{tabular}{lllllllllllllllllllll}
\hline 30 & 20 & 10 & 0 & -10 & -20 & -30 & -40 & -50 & -60 & -70 & -80 & -90 & -110 & -130 & -150 & -170 & -190 \\
& & & & &
\end{tabular}

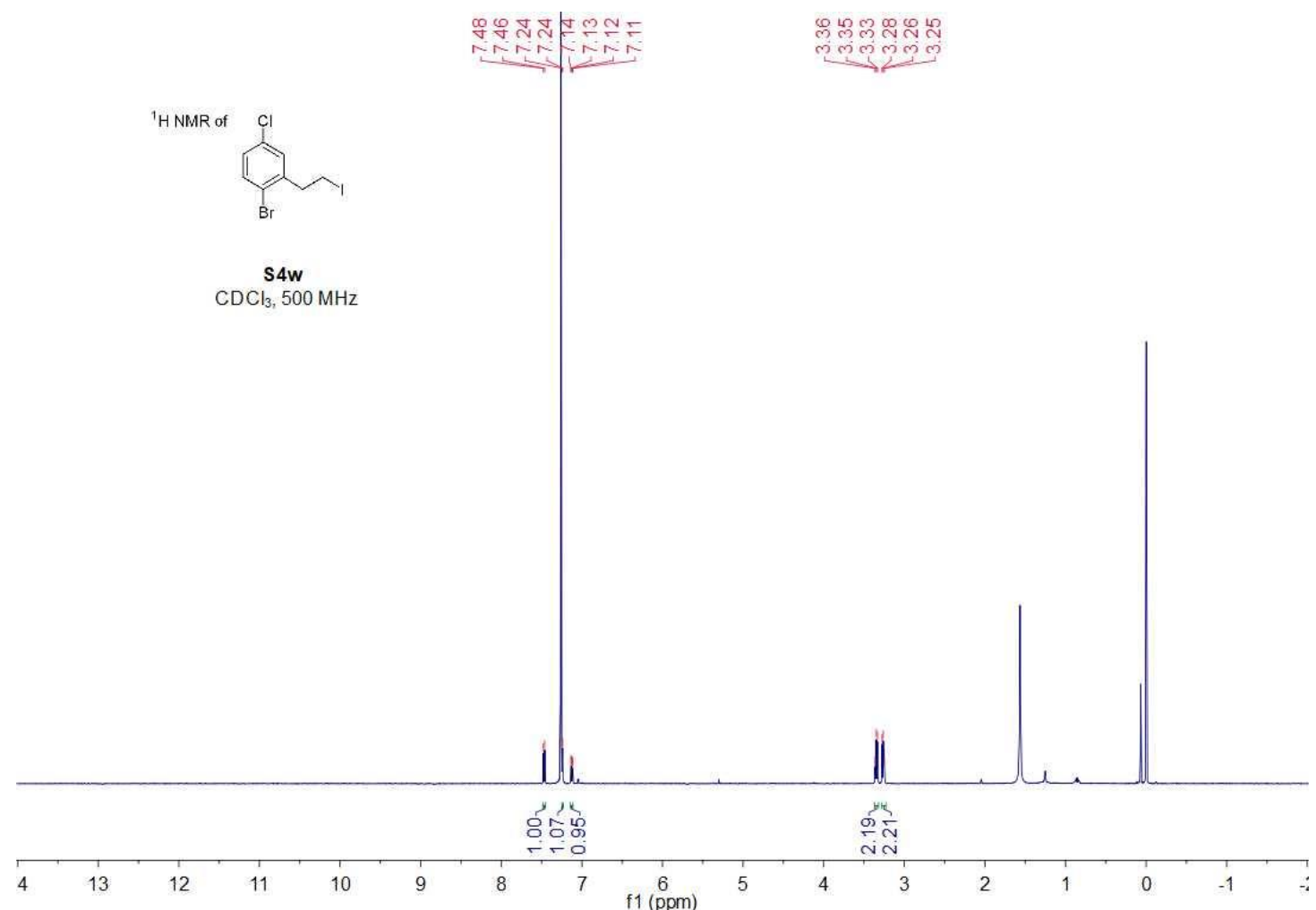



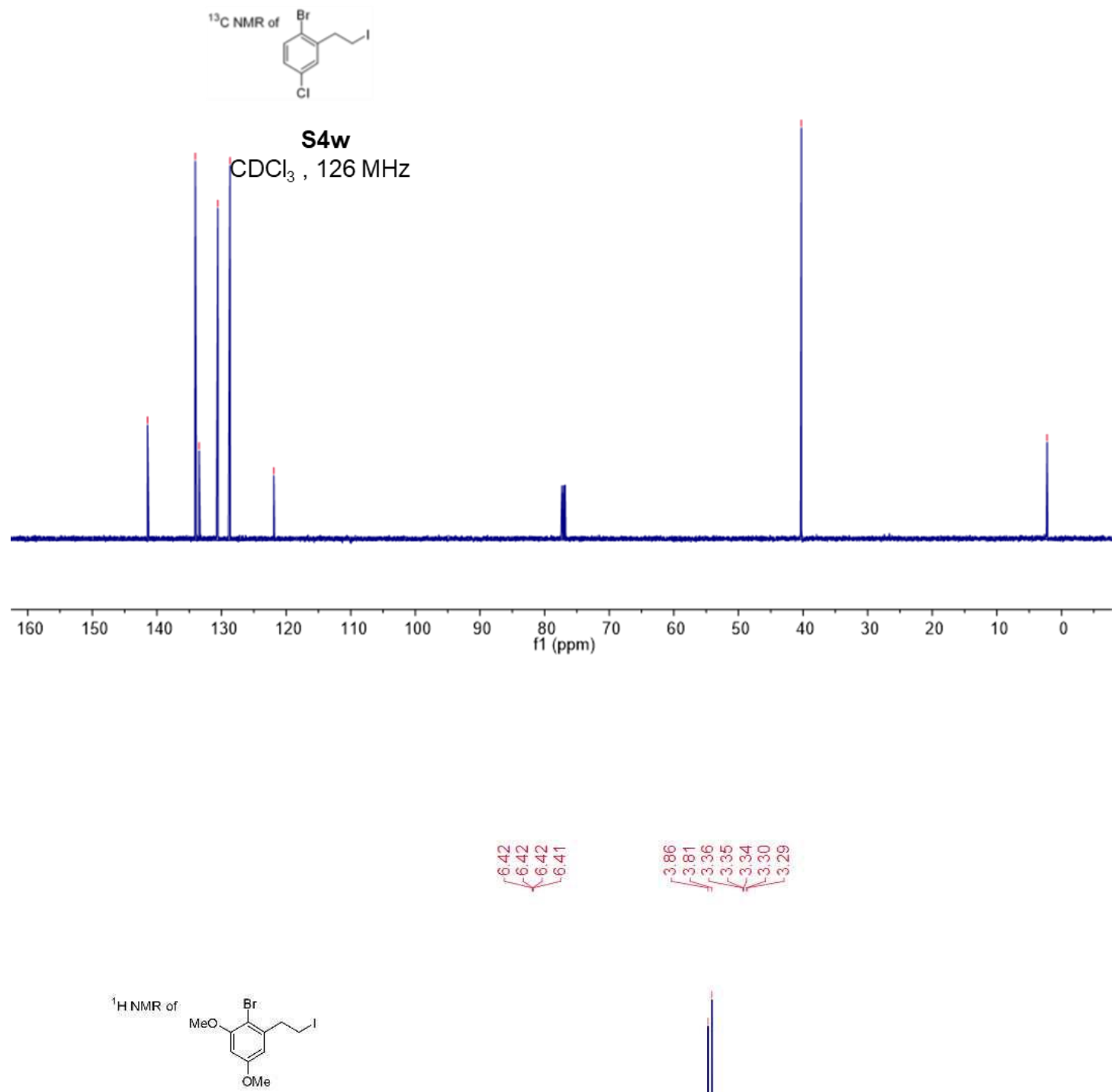

S4z

$\mathrm{CDCl}_{3}, 500 \mathrm{MHz}$

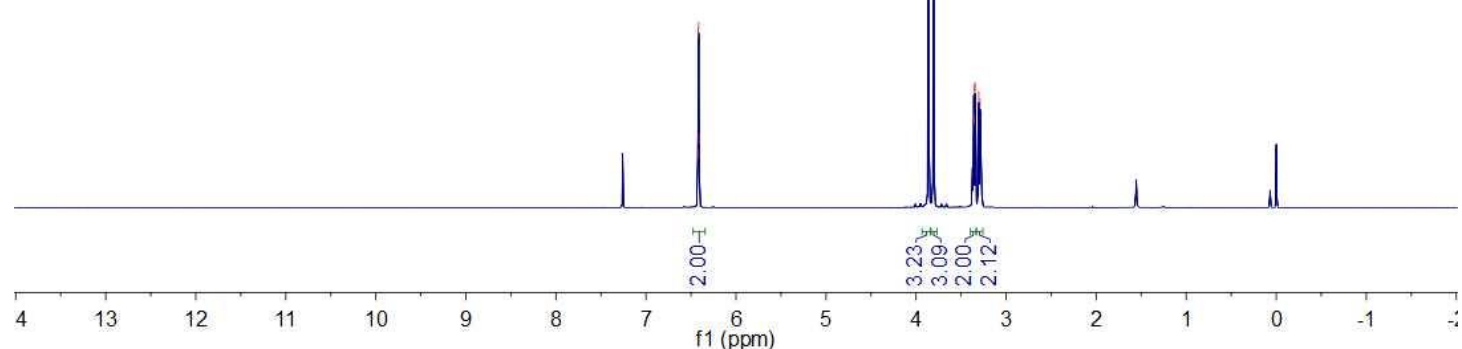




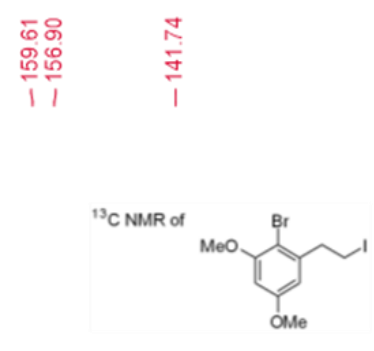

मूल
ㅎํ

लํㅛ

용

$\mathrm{CDCl}_{3}, 126 \mathrm{MHz}$

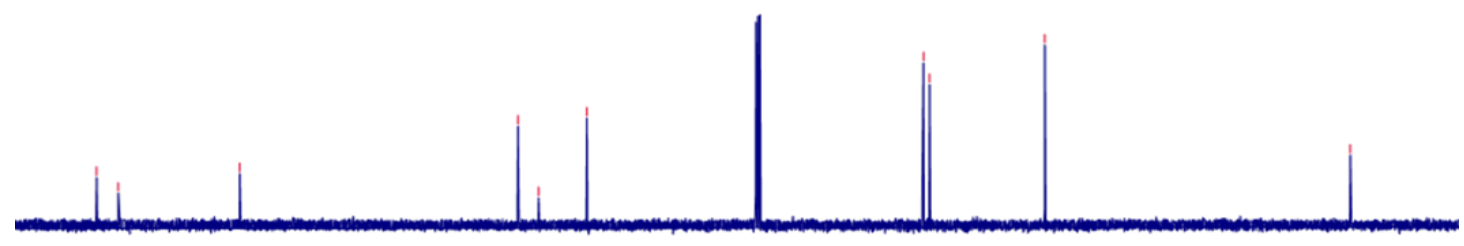

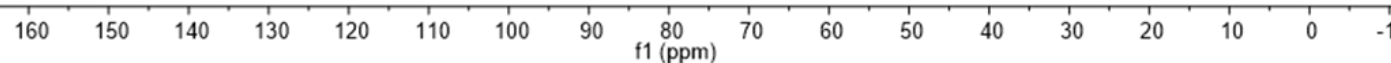

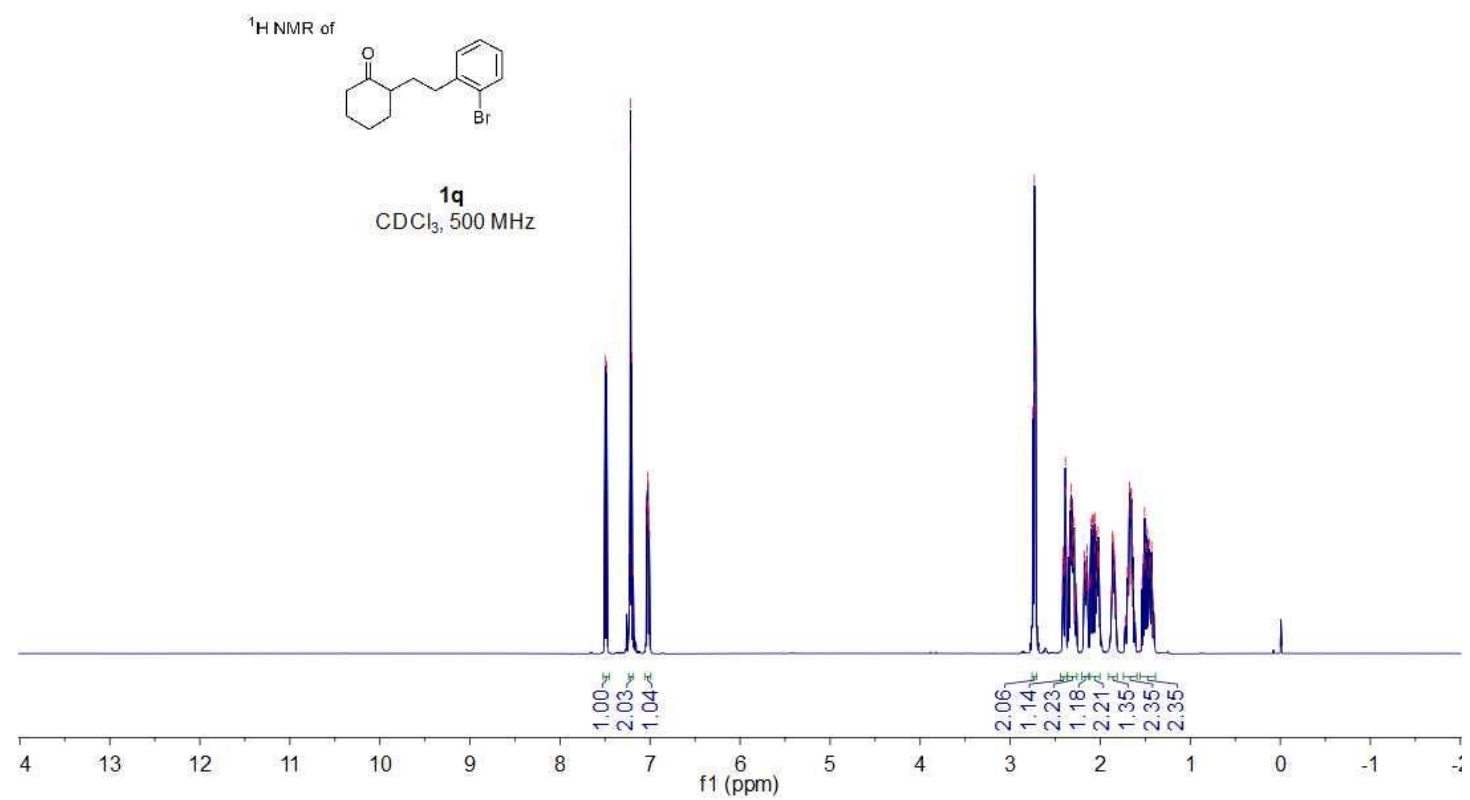




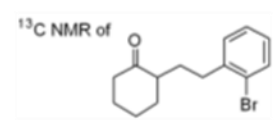

$1 \mathrm{q}$

$\mathrm{CDCl}_{3}, 126 \mathrm{MHz}$

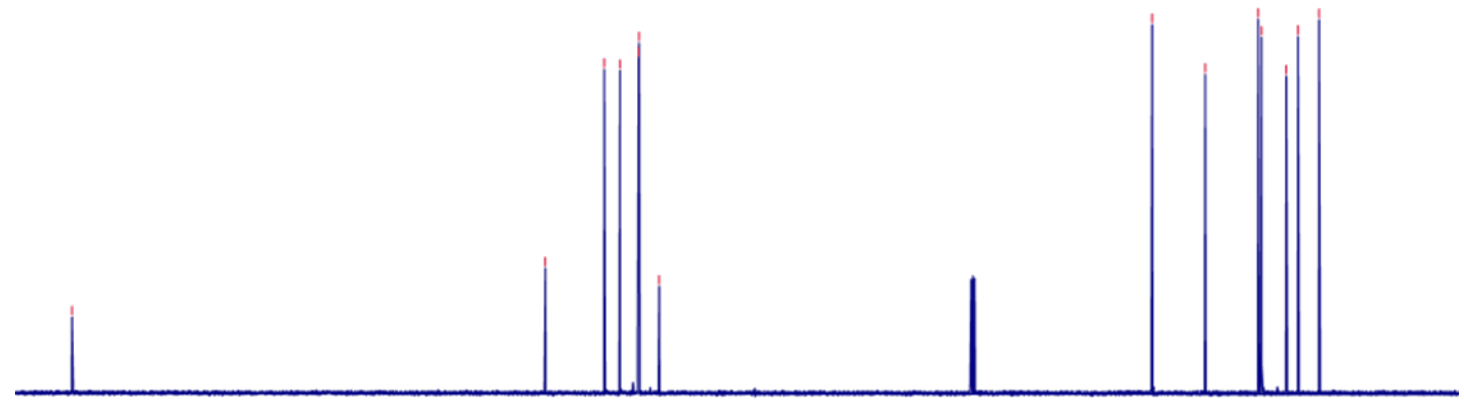

\begin{tabular}{lllllllllllllllllllllllllll}
\hline 20 & 210 & 200 & 190 & 180 & 170 & 160 & 150 & 140 & 130 & 120 & 110 & 100 & 90 & 80 & 70 & 60 & 50 & 40 & 30 & 20 & 10
\end{tabular}

${ }^{1}$ HNMR of<smiles>COc1ccc(Br)c(CCC2CCCCC2=O)c1</smiles>

$\mathrm{CDCl}_{3}, 500 \mathrm{MHz}$

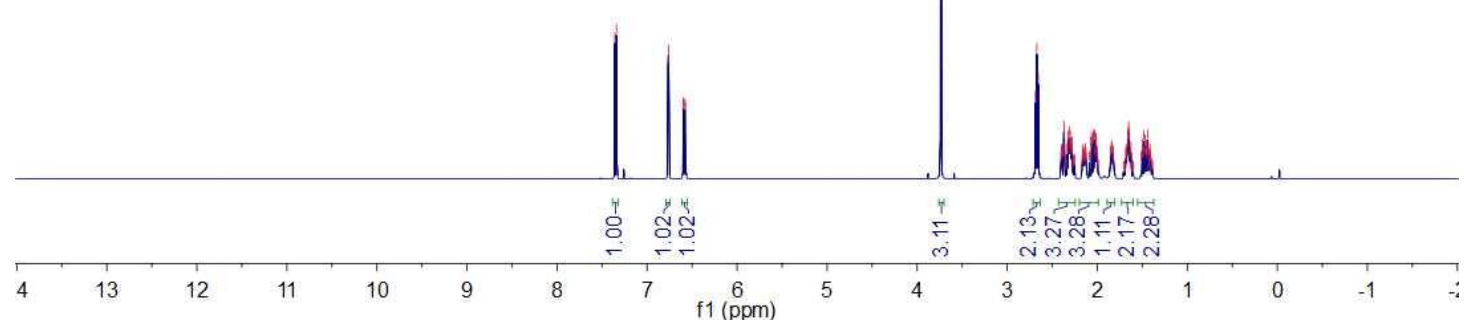




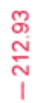

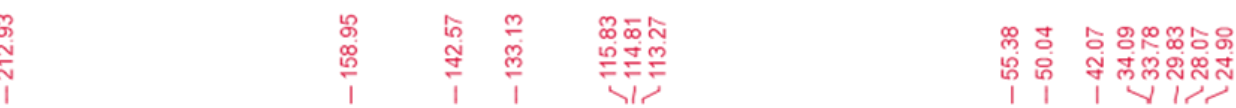

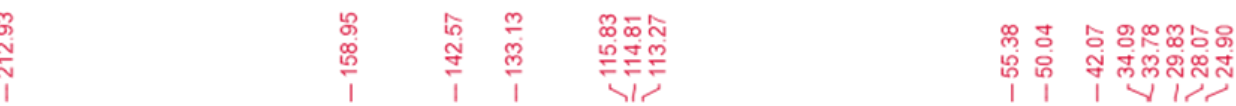

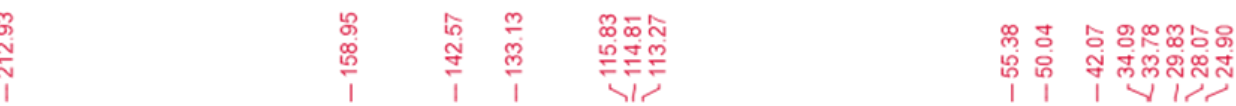

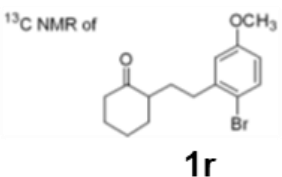

$\mathrm{CDCl}_{3}, 126 \mathrm{MHz}$

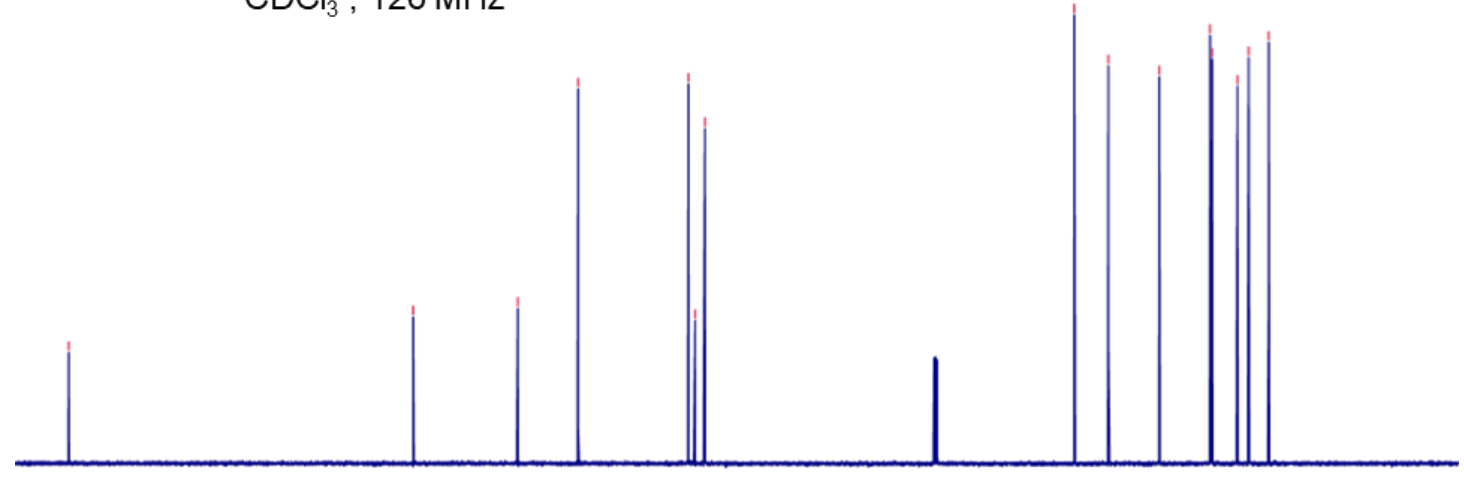

$\begin{array}{llllllllllllllllllllllllllllllllllllllll}120 & 210 & 200 & 190 & 180 & 170 & 160 & 150 & 140 & 130 & 120 & 110 & 100 & 90 & 80 & 70 & 60 & 50 & 40 & 30 & 20 & 10 & 0\end{array}$<smiles>O=C1CCCCC1CCc1cc(F)ccc1Br</smiles>

is

$\mathrm{CDCl}_{3}, 500 \mathrm{MHz}$

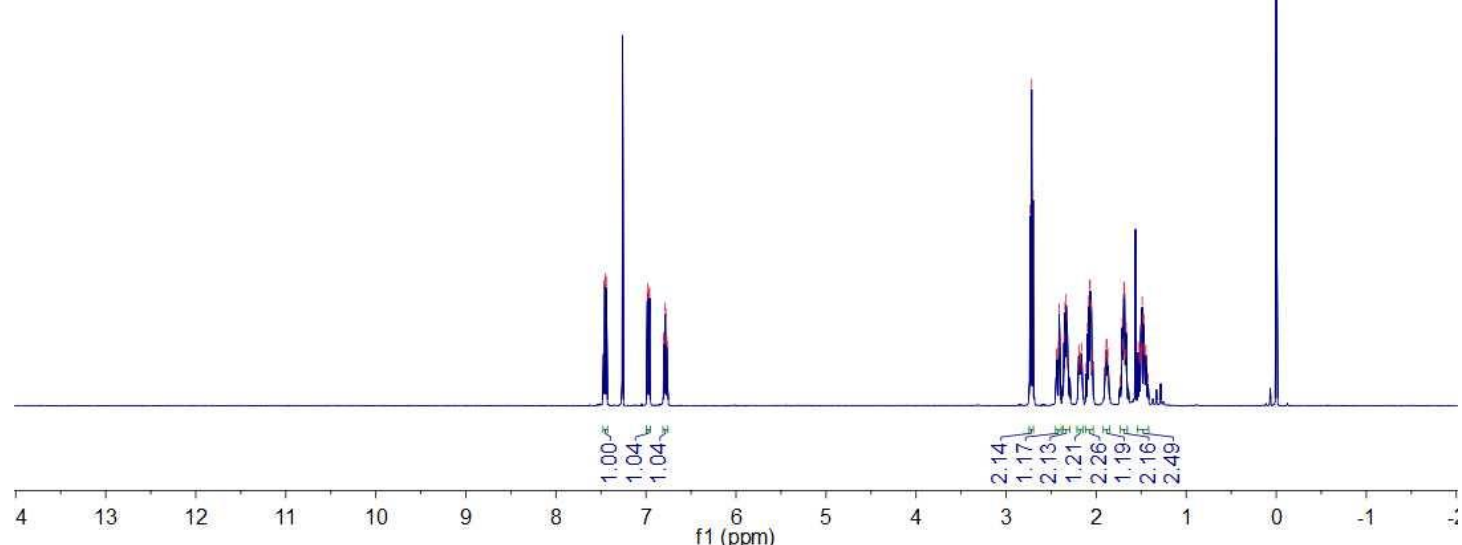


${ }^{13} \mathrm{C}$ NMR of

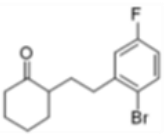

1s

$\mathrm{CDCl}_{3}, 126 \mathrm{MHz}$
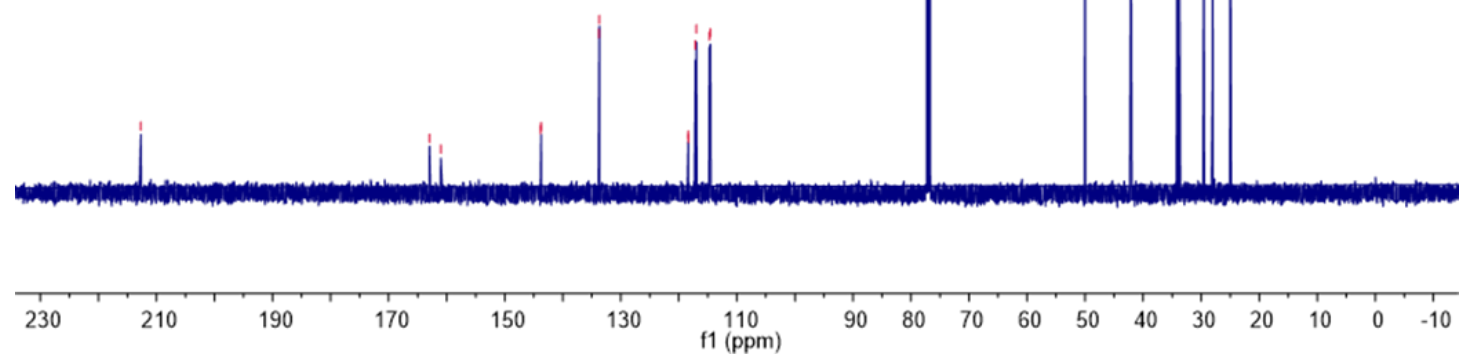

${ }^{19} \mathrm{~F}$ NMR of

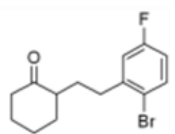

$1 \mathrm{~s}$

$\mathrm{CDCl}_{3}, 376 \mathrm{MHz}$

$\begin{array}{lllllllllllll}30 & 20 & 10 & 0 & -10 & -20 & -30 & -40 & -50 & -60 & -70 & -80 & -90 \\ & & & & & & & & & & & \end{array}$ 
${ }^{1} \mathrm{H} N M R$ of<smiles>COc1ccc(CCC2CCCCC2=O)c(Br)c1</smiles>

$\stackrel{\text { 1t }}{\mathrm{CDCl}_{3}, 500 \mathrm{MHz}}$

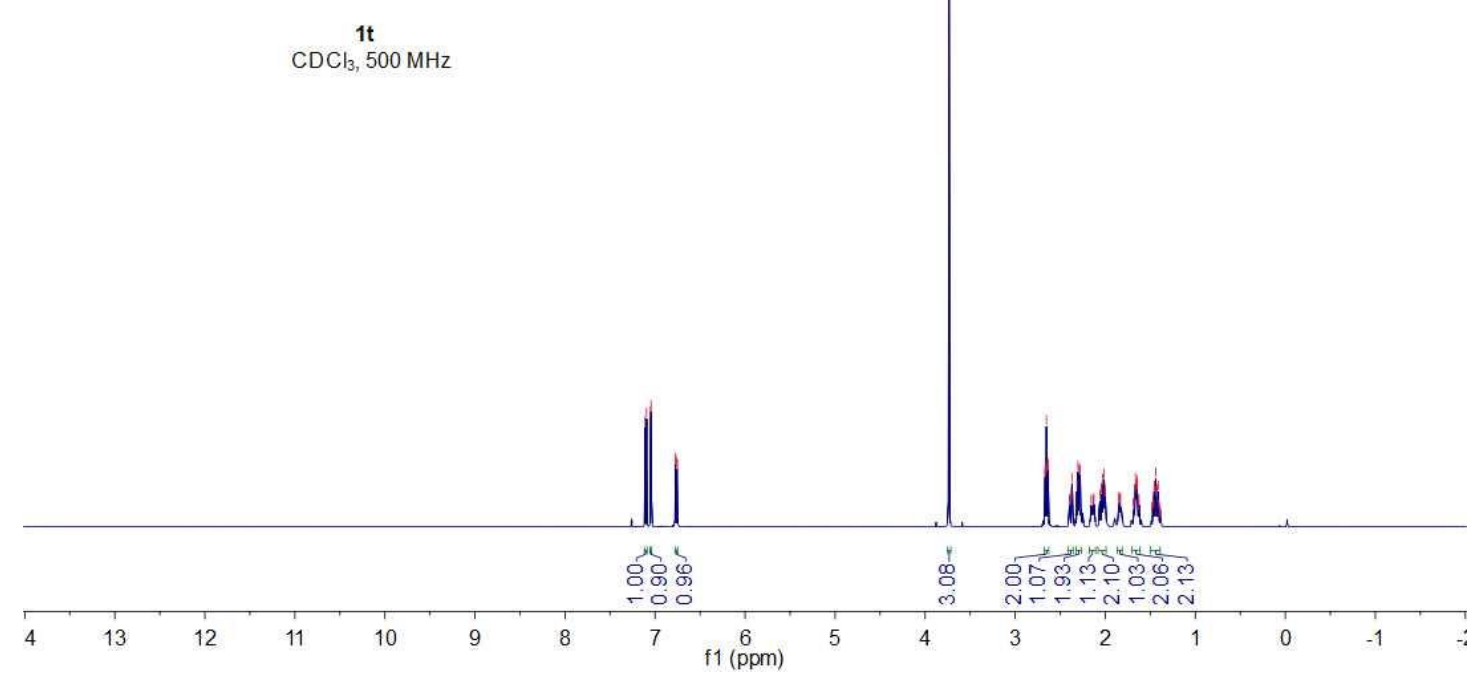

\begin{tabular}{|c|c|c|c|}
\hline 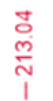 & $\underset{\substack{N \\
\infty}}{\stackrel{\leftrightarrow}{\leftarrow}}$ & 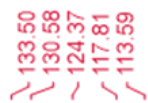 & 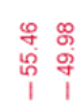 \\
\hline
\end{tabular}

${ }^{13} \mathrm{C}$ NMR of<smiles>COc1ccc(CCC2CCCCC2=O)c(Br)c1</smiles>

$1 \mathbf{t}$

$\mathrm{CDCl}_{3}, 126 \mathrm{MHz}$

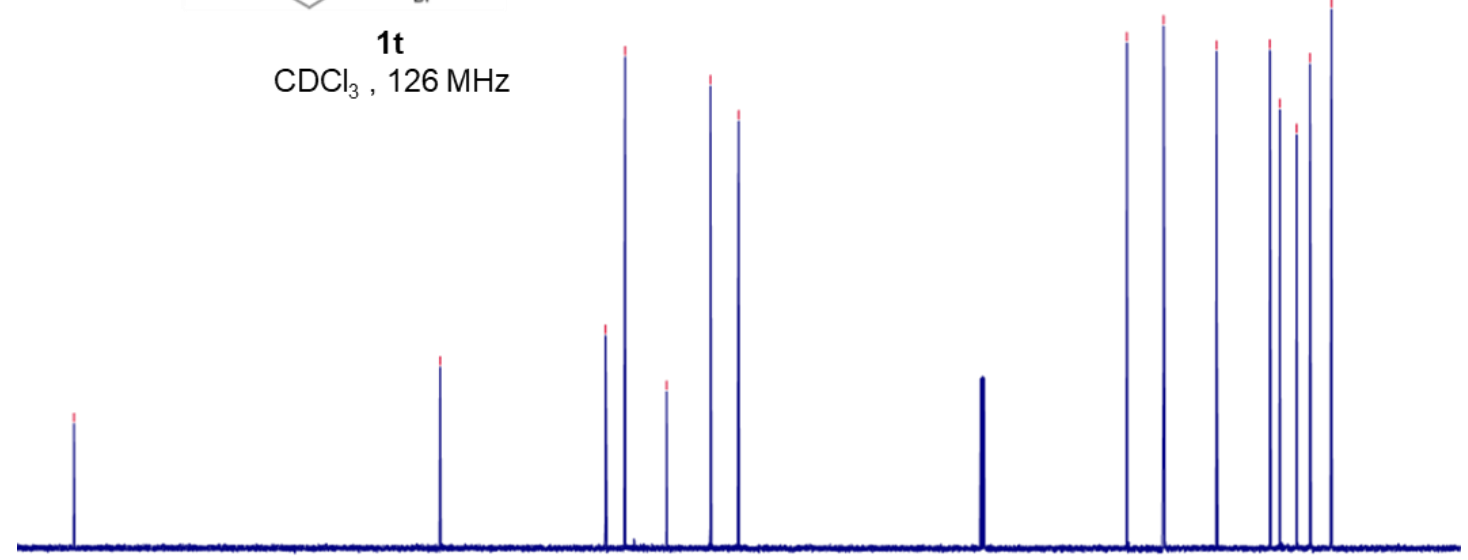
$\begin{array}{lllllllllllllllllll}120 & 210 & 200 & 190 & 180 & 170 & 160 & 150 & 140 & 130 & 120 & 1 & 1 & 1 & 1 \\ \mathrm{f} 1(\mathrm{ppm}) & 100 & 90 & 80 & 70 & 60 & 50 & 40 & 30 & 20 & 10\end{array}$ 
${ }^{1}$ HNMR of<smiles>CC(CCc1ccccc1)CCc1ccccc1</smiles>

$1 \mathrm{u}$

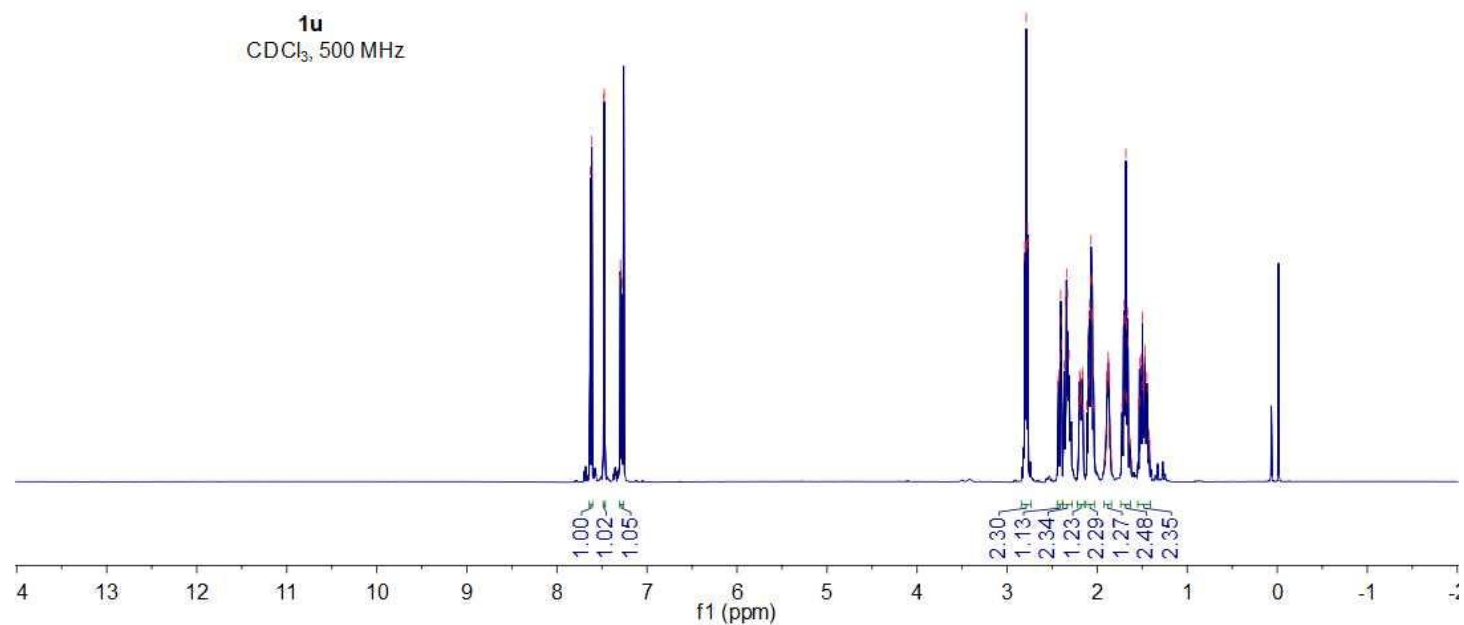

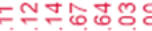
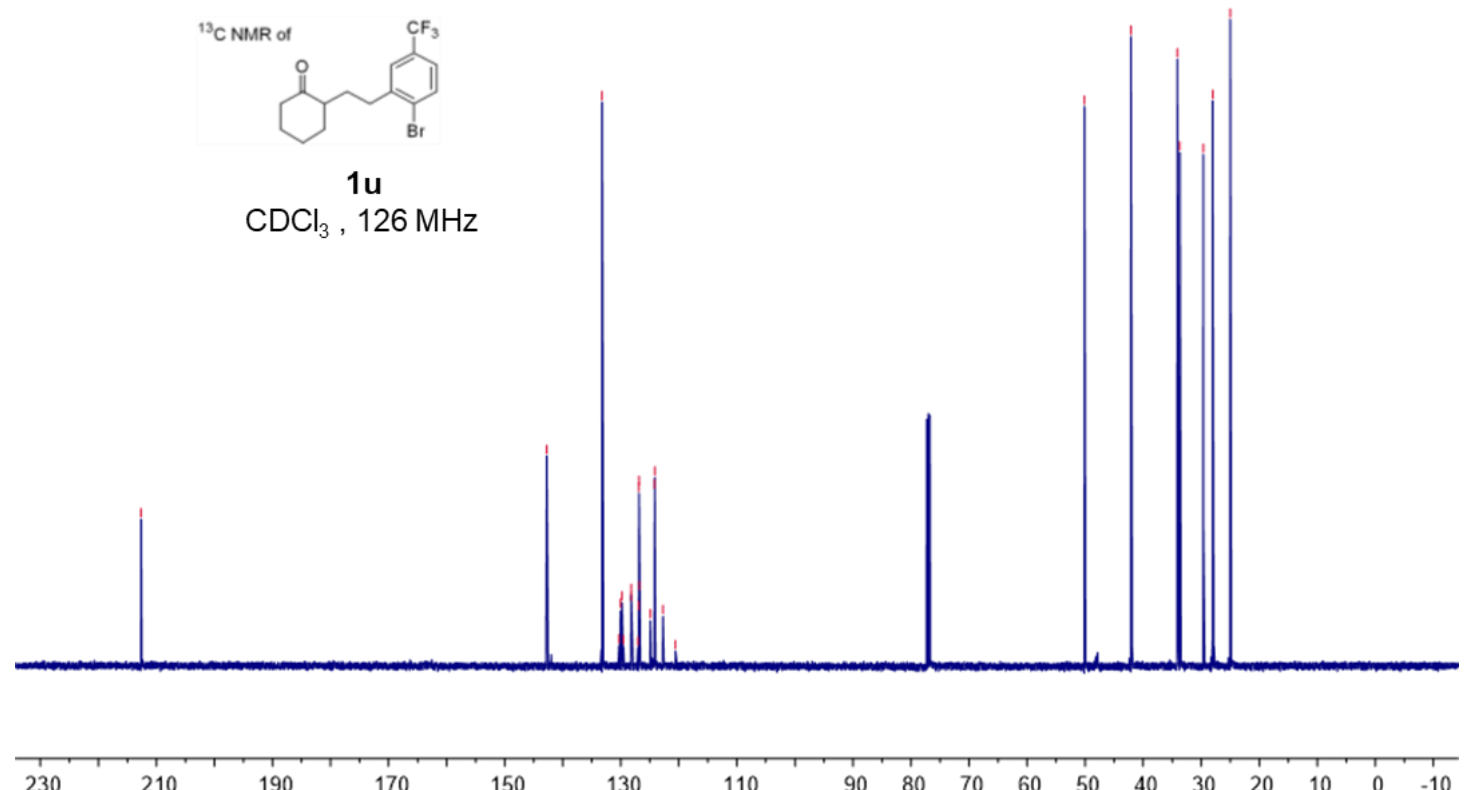

130 110

$\begin{array}{lllllllllll}90 & 80 & 70 & 60 & 50 & 40 & 30 & 20 & 10 & 0 & -10\end{array}$ 


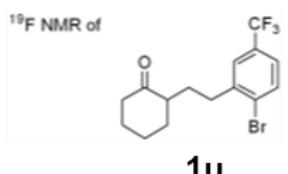

$\mathrm{CDCl}_{3}, 376 \mathrm{MHz}$

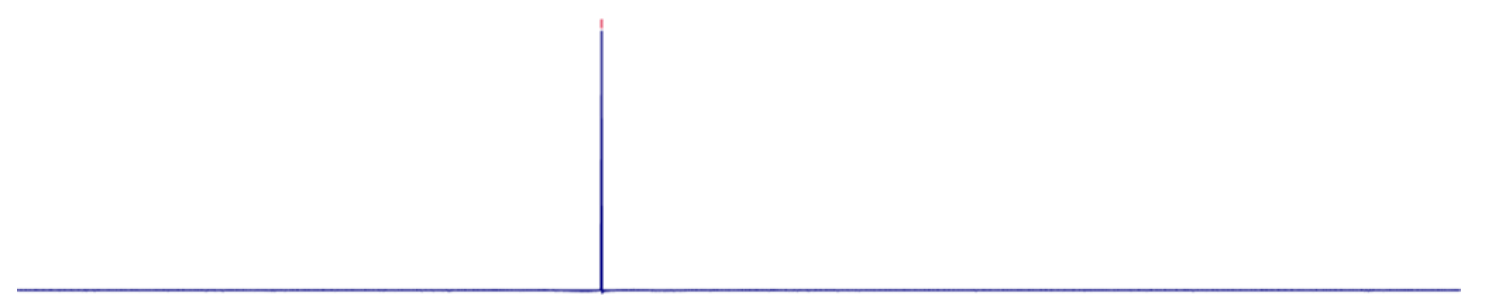

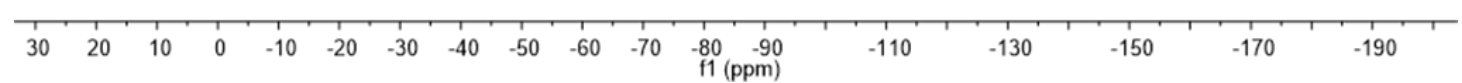

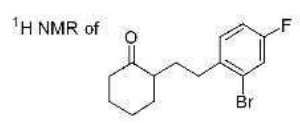

$1 v$

$\mathrm{CDCl}_{3}, 500 \mathrm{MHz}$

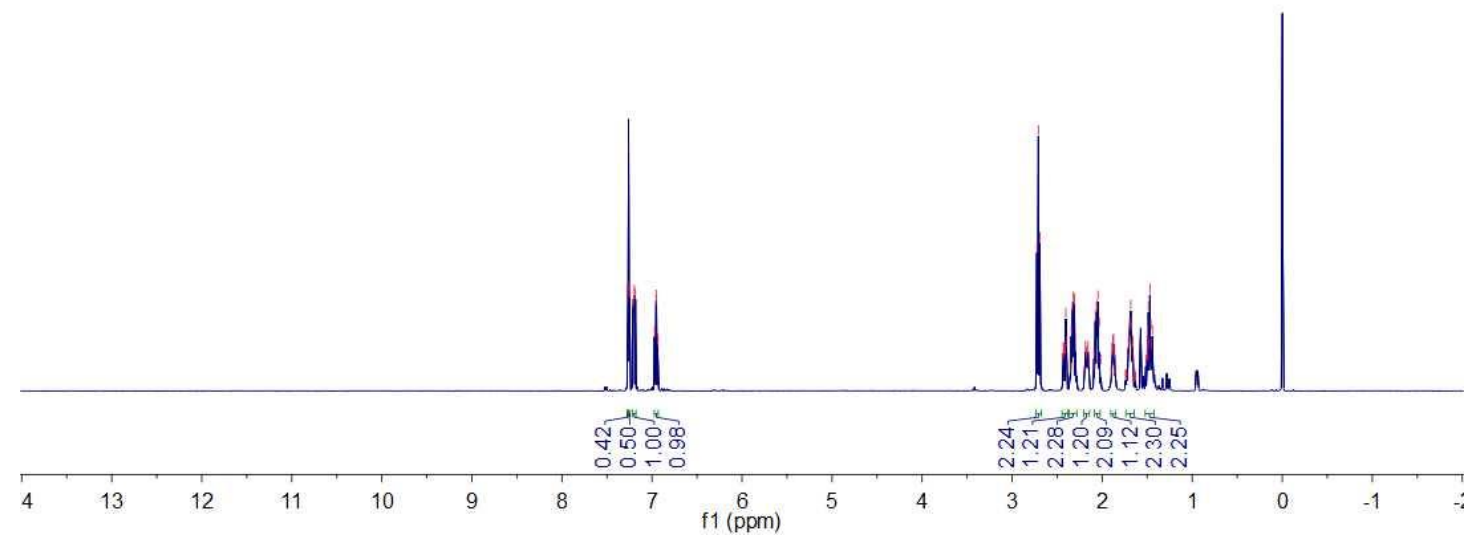



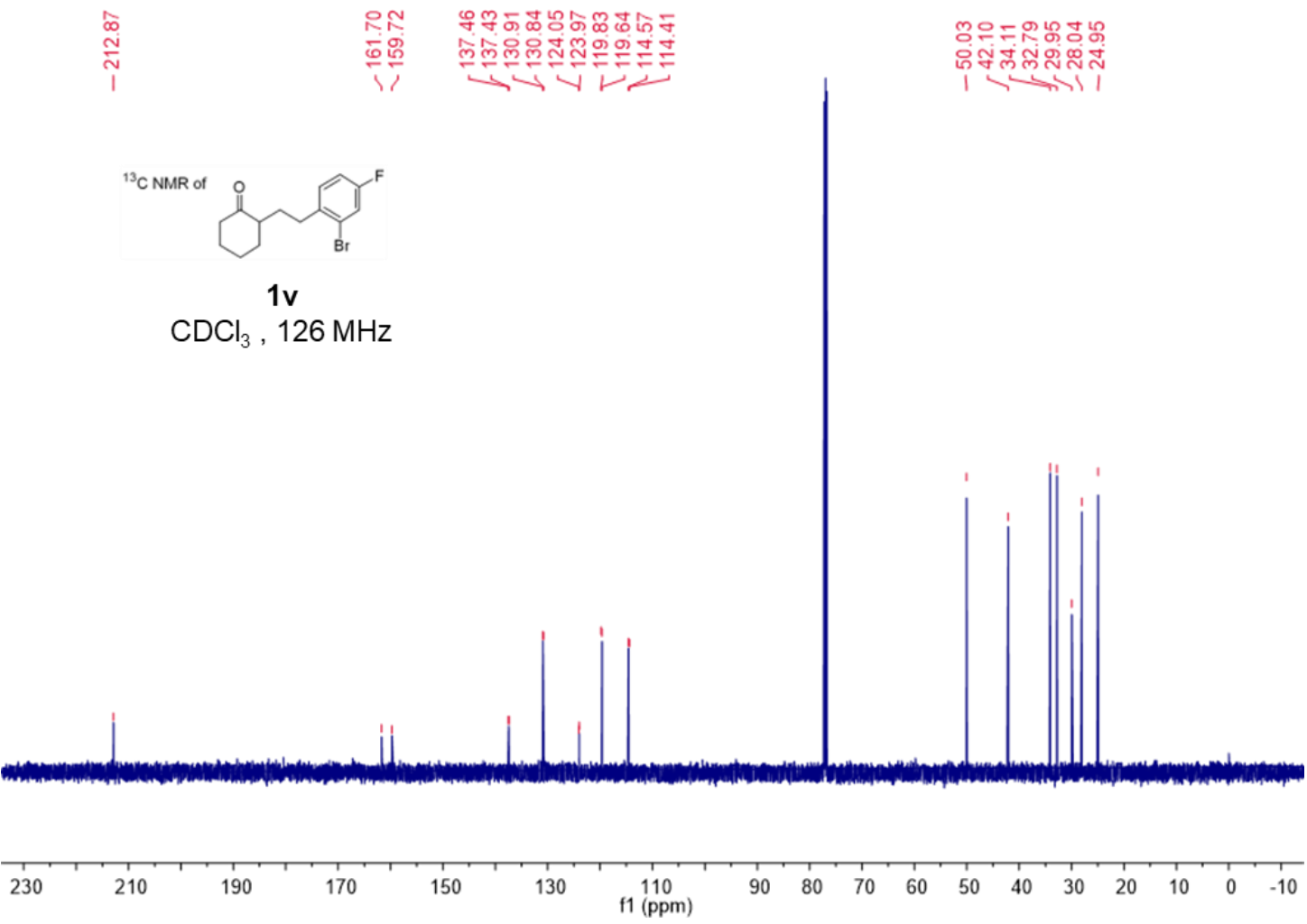

$$
\frac{\mathfrak{7}}{\frac{\mathfrak{5}}{\mathrm{i}}}
$$

${ }^{19} \mathrm{~F}$ NMR of

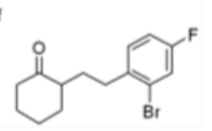

$1 v$

$\mathrm{CDCl}_{3}, 376 \mathrm{MHz}$

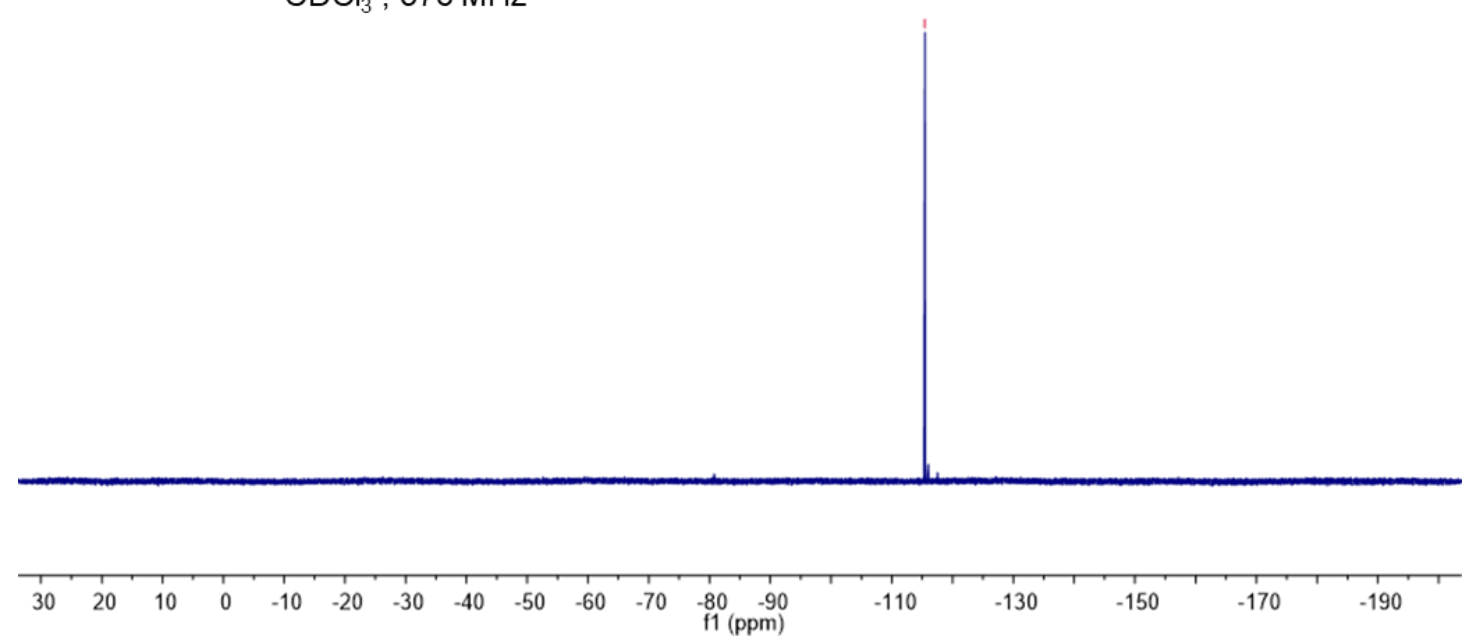



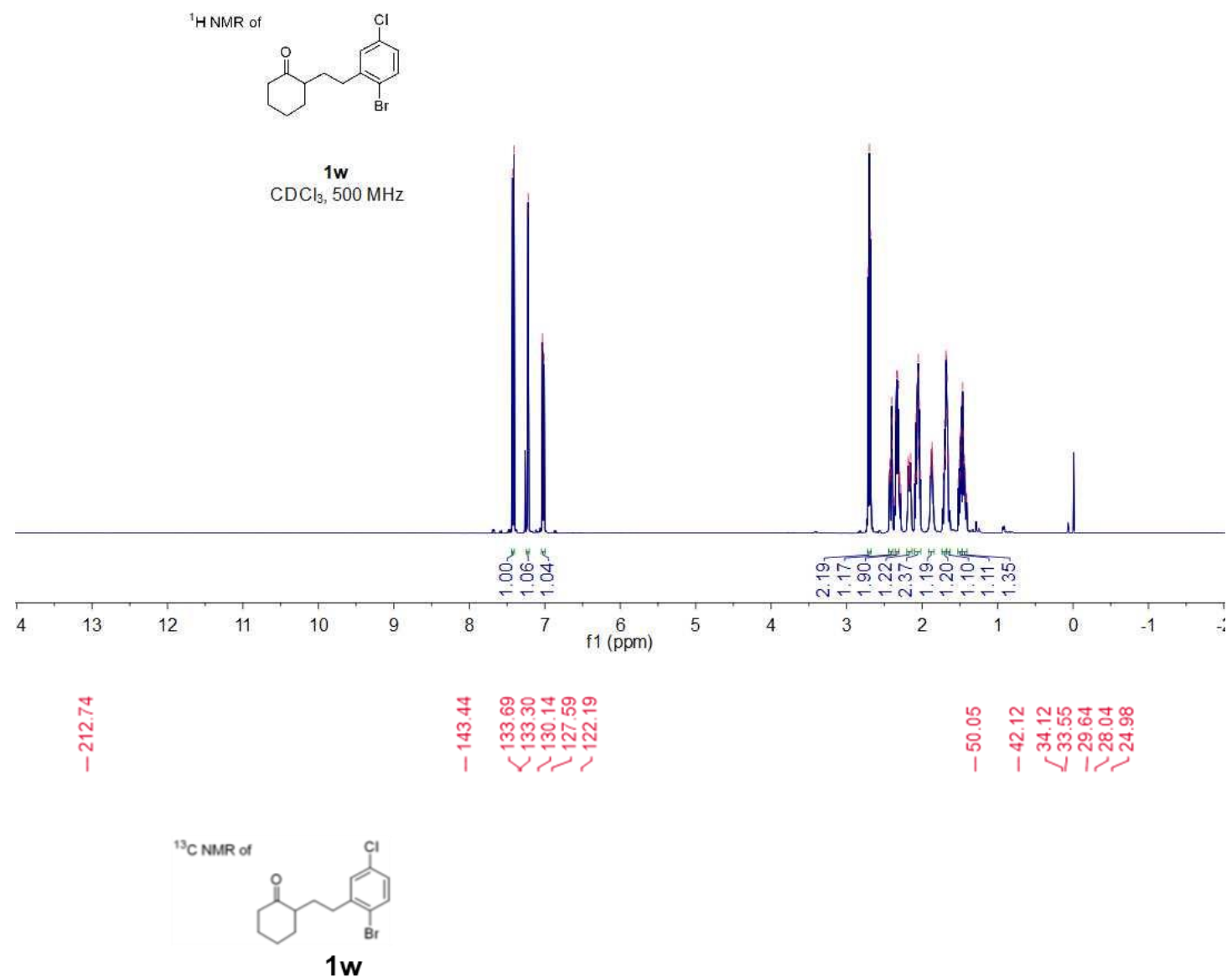

$\mathrm{CDCl}_{3}, 126 \mathrm{MHz}$

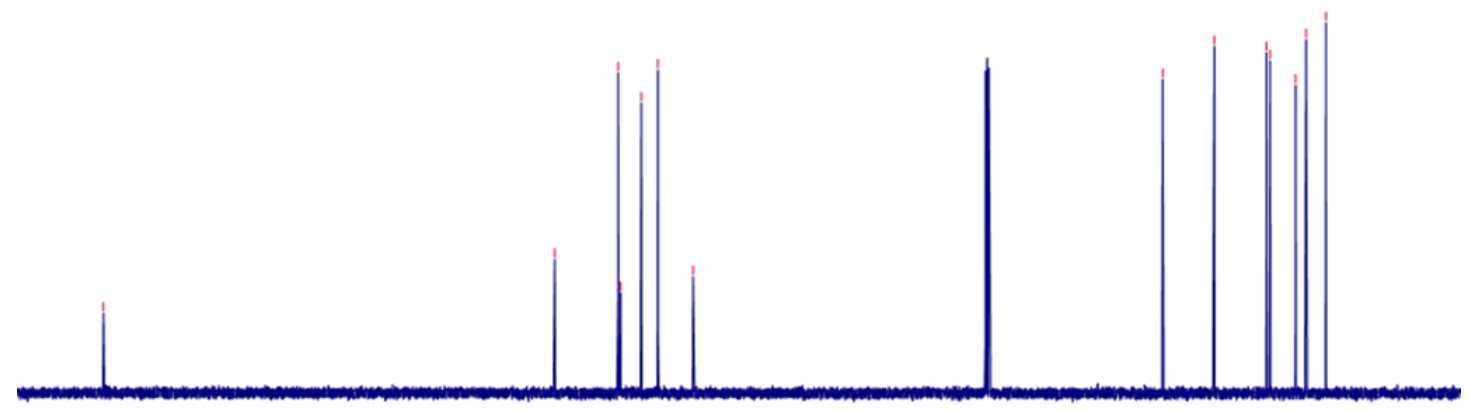

$\begin{array}{lllllllllllllllllllllllll}1 & 120 & 210 & 200 & 190 & 180 & 170 & 160 & 150 & 140 & 130 & 120 & 110 & 100 & 90 & 80 & 70 & 60 & 50 & 40 & 30 & 20 & 10\end{array}$ 

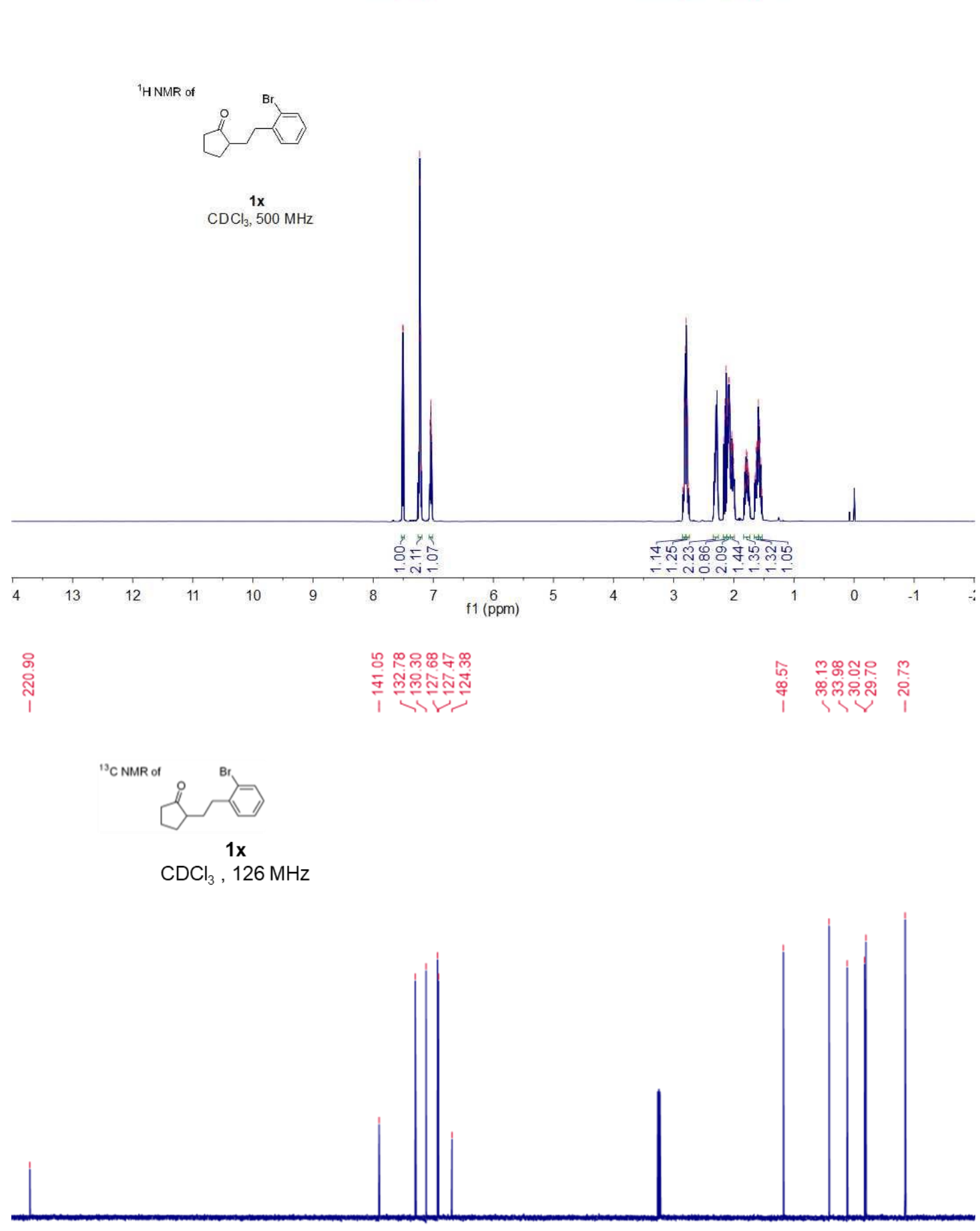

$\begin{array}{llllllllllllllllllllllllll}220 & 210 & 200 & 190 & 180 & 170 & 160 & 150 & 140 & 130 & 120 & 110 & 100 & 90 & 80 & 70 & 60 & 50 & 40 & 30 & 20 & 10\end{array}$ 

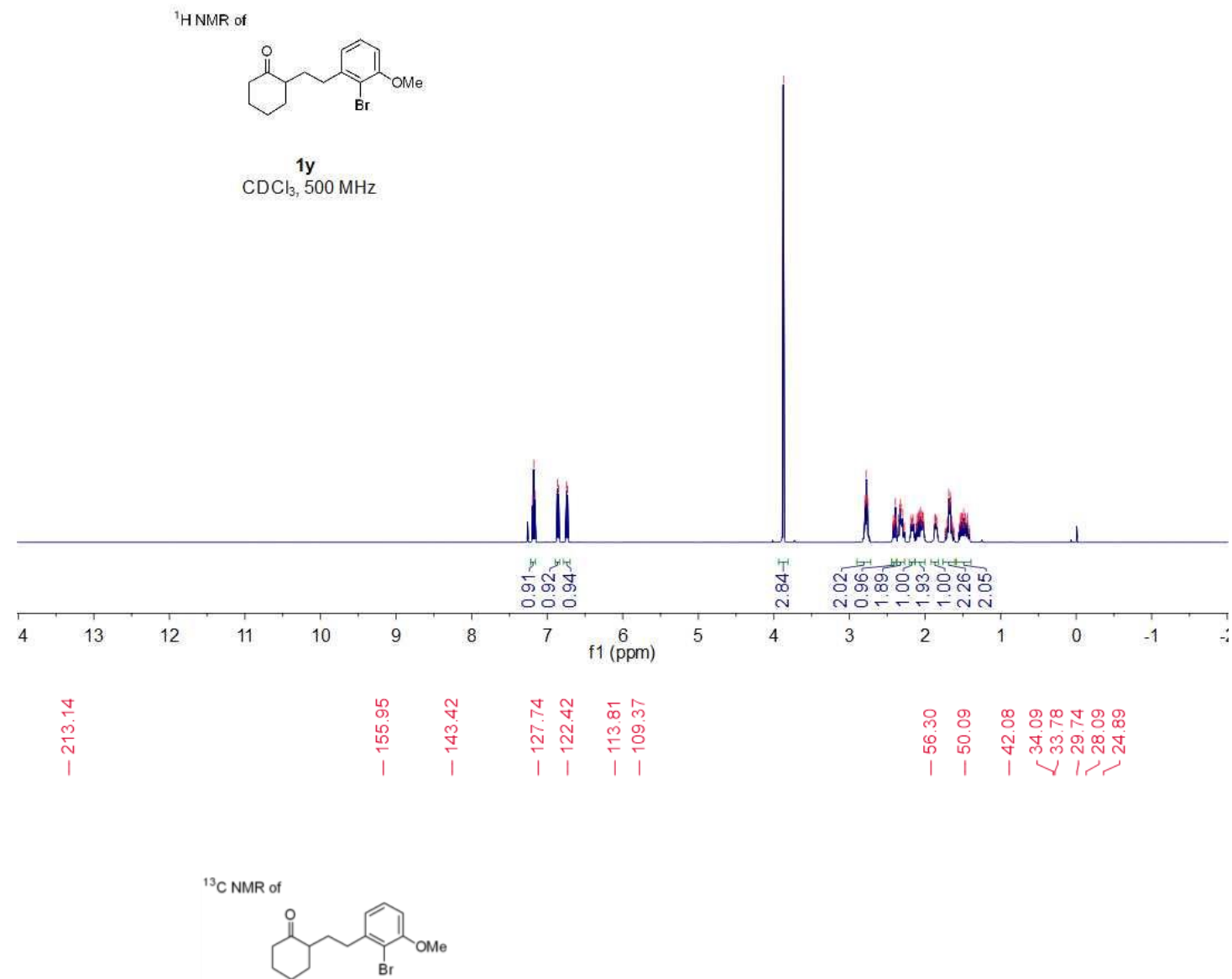

$1 y$

$\mathrm{CDCl}_{3}, 126 \mathrm{MHz}$

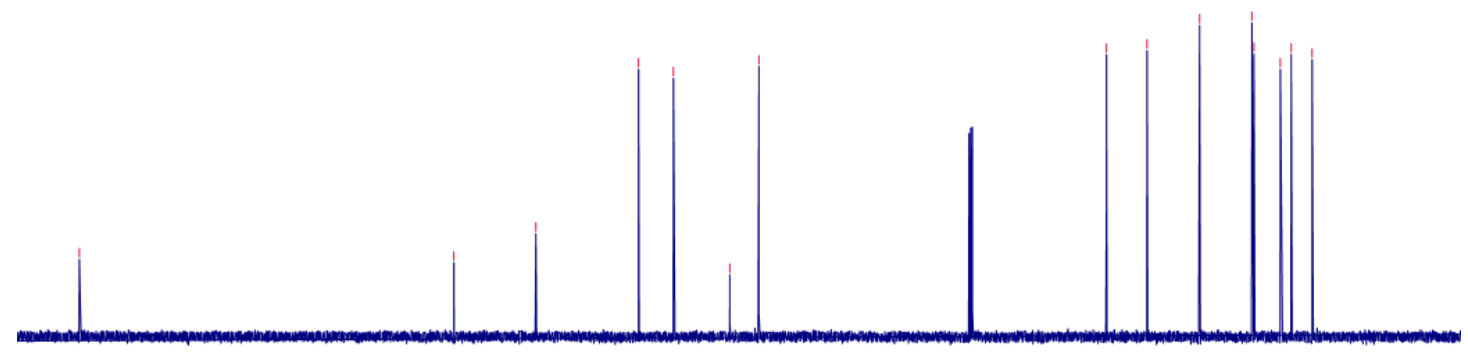

$\begin{array}{llllllllllllllllllllll}220 & 210 & 200 & 190 & 180 & 170 & 160 & 150 & 140 & 130 & 120 & 110 & 100 & 90 & 80 & 70 & 60 & 50 & 40 & 30 & 20 & 10\end{array}$ 

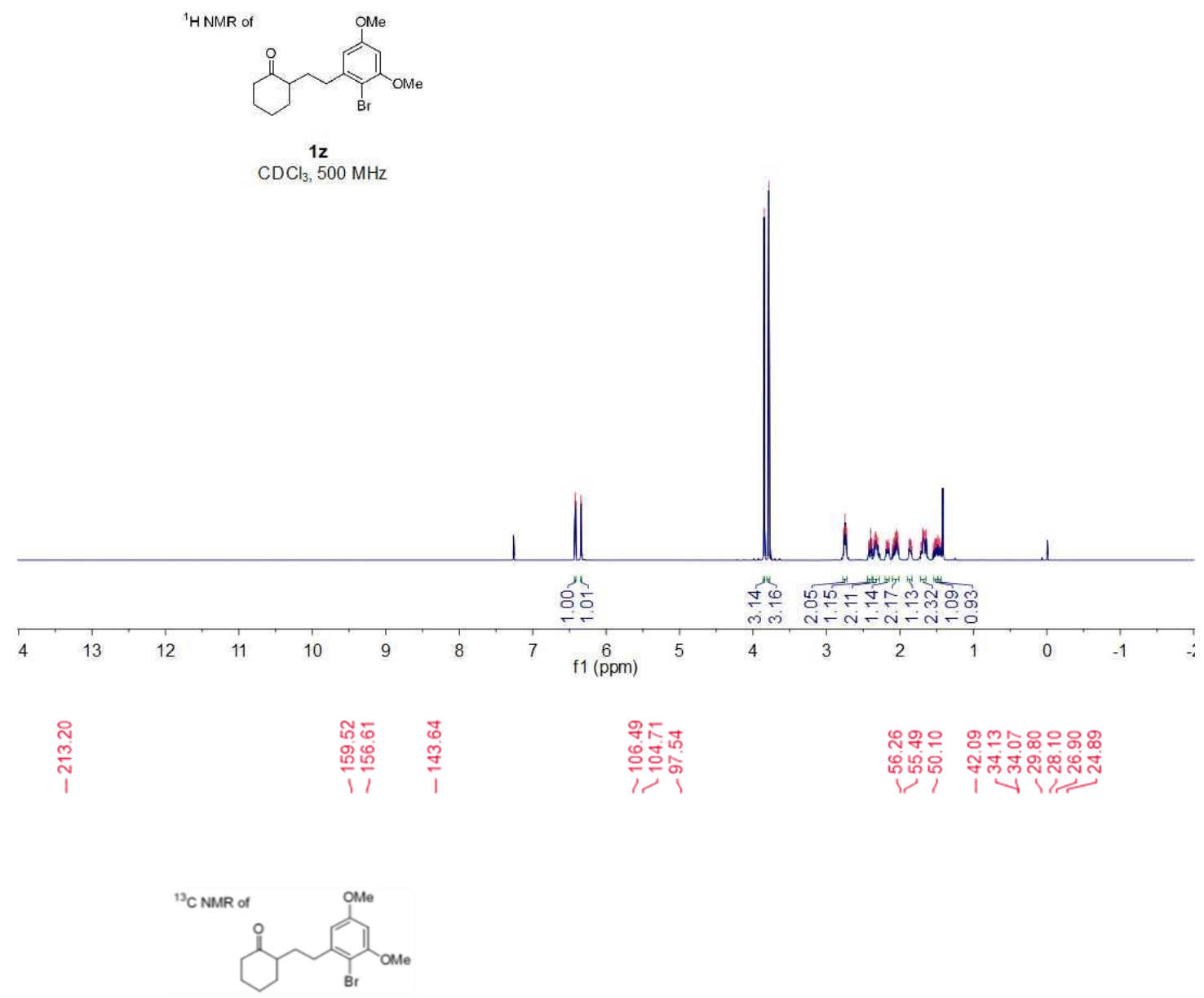

$1 \mathrm{z}$

$\mathrm{CDCl}_{3}, 126 \mathrm{MHz}$

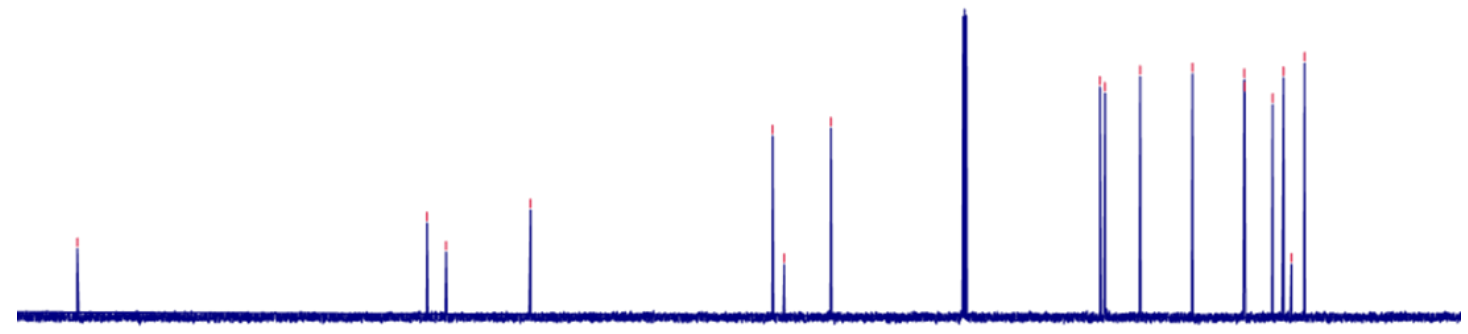

$\begin{array}{llllllllllllllllllllllllllllllll}1 & 120 & 210 & 200 & 190 & 180 & 170 & 160 & 150 & 140 & 130 & 120 & 110 & 100 & 90 & 80 & 70 & 60 & 50 & 40 & 30 & 20 & 10\end{array}$ 

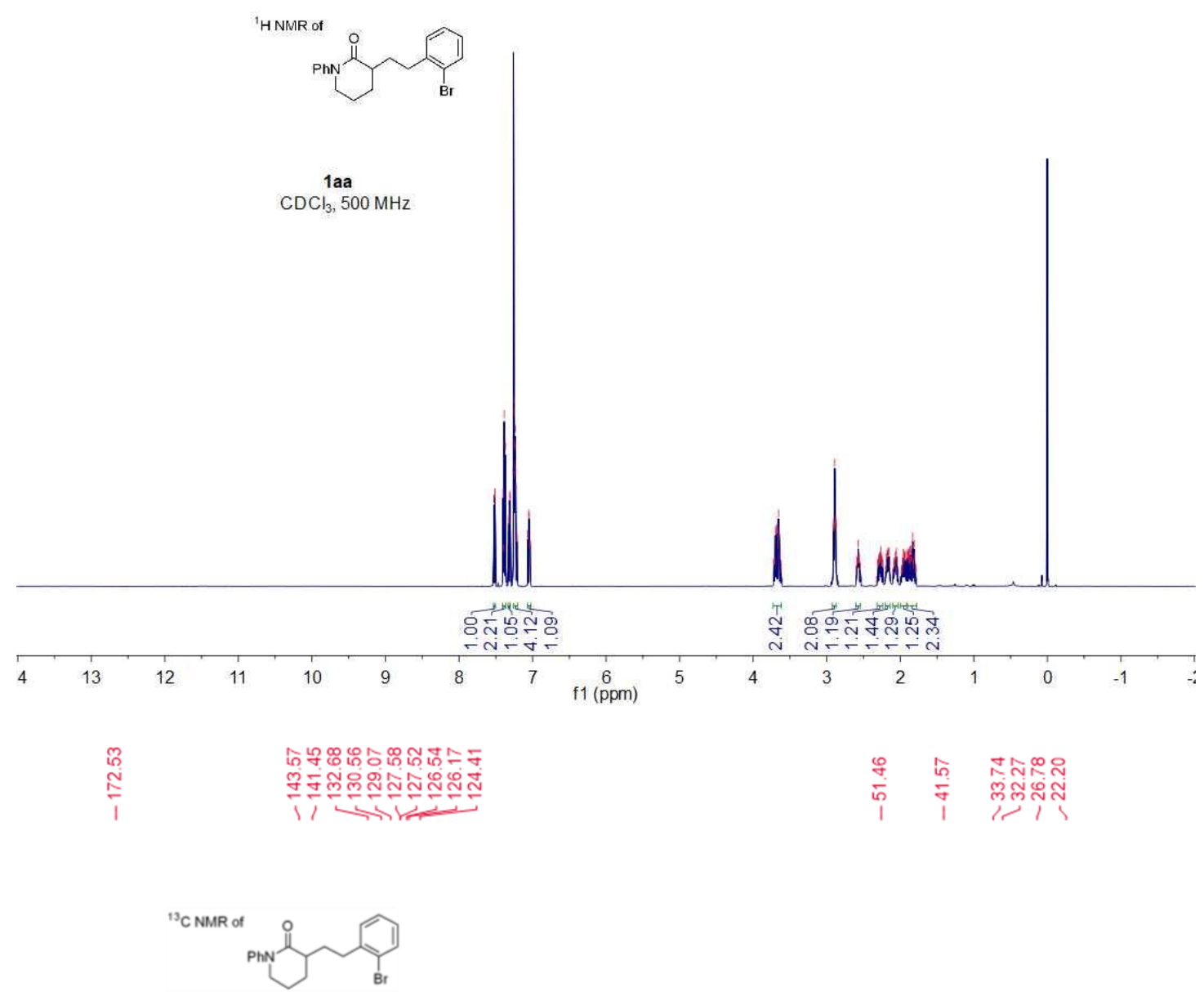

1 aa

$\mathrm{CDCl}_{3}, 126 \mathrm{MHz}$

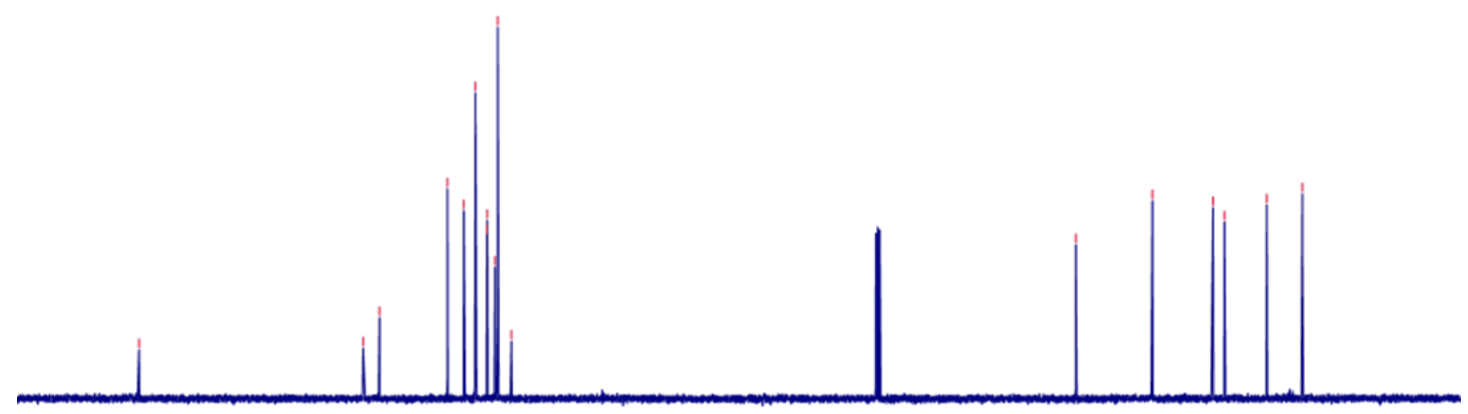

$\begin{array}{lllllllllllllllll}180 & 170 & 160 & 150 & 140 & 130 & 120 & 110 & \begin{array}{l}100 \\ \mathrm{f} 1(\mathrm{ppm})\end{array} & 80 & 70 & 60 & 50 & 40 & 30 & 20 & 10\end{array}$ 


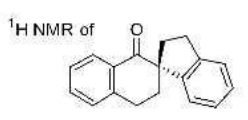

2a

$\mathrm{CDCl}_{3}, 500 \mathrm{MHz}$

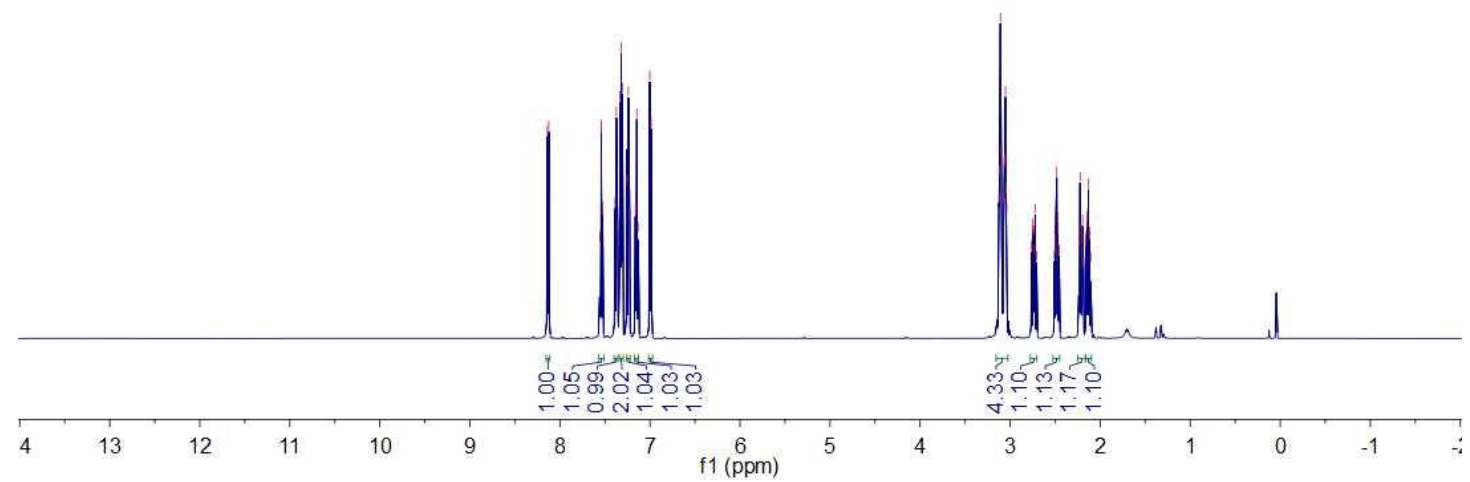

\begin{tabular}{|c|c|c|}
\hline 饮 & 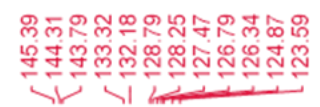 & $\underset{\substack{9 \\
\infty \\
\infty \\
\infty \\
1}}{\substack{0 \\
1}}$ \\
\hline
\end{tabular}

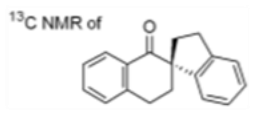

$2 a$

$\mathrm{CDCl}_{3}, 126 \mathrm{MHz}$

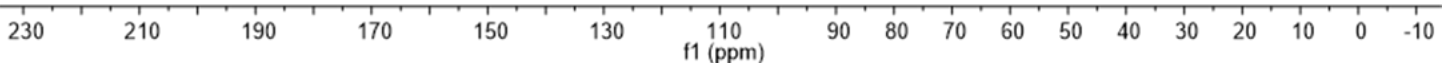




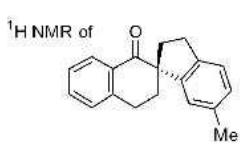

$2 b$

$\mathrm{CDCl}_{3}, 500 \mathrm{MHz}$

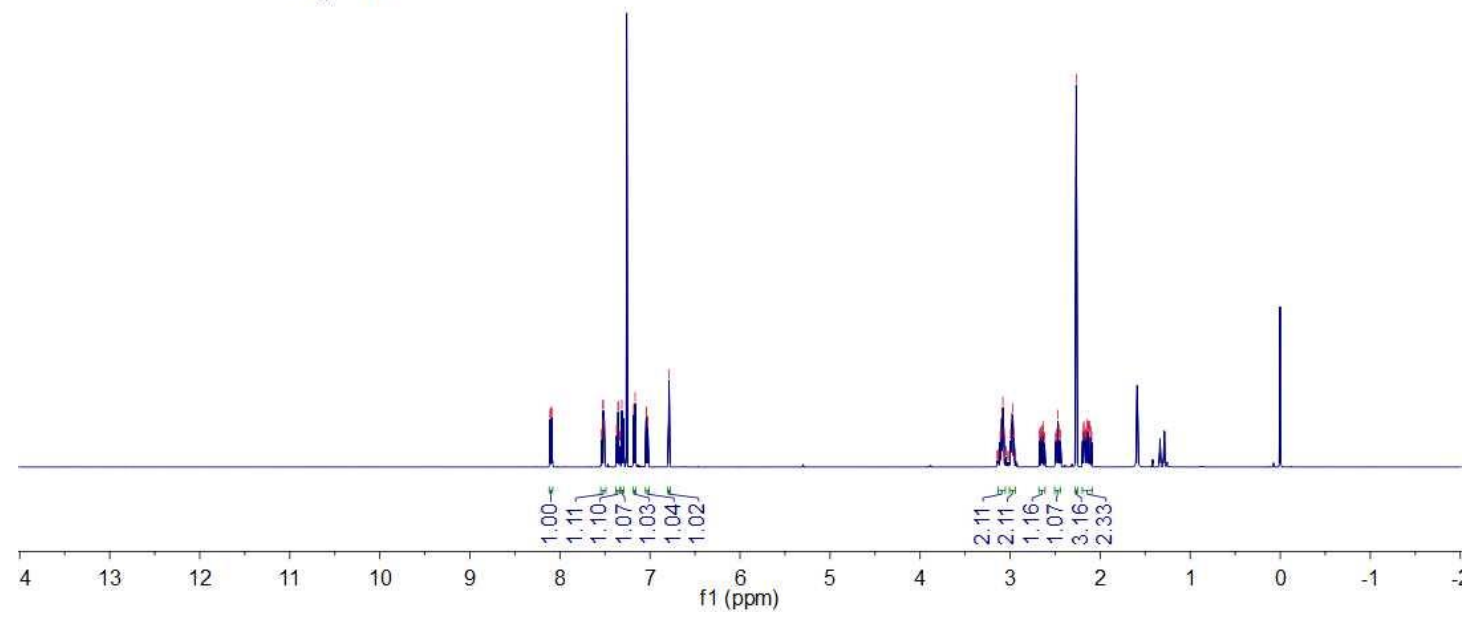

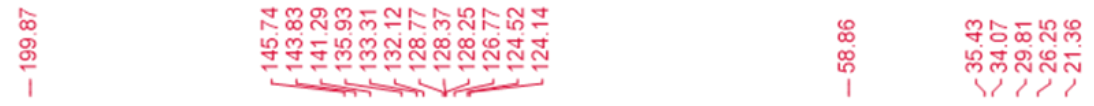

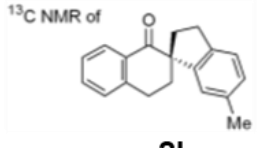

$2 b$

$\mathrm{CDCl}_{3}, 126 \mathrm{MHz}$

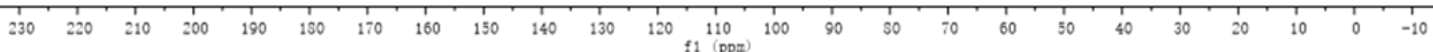




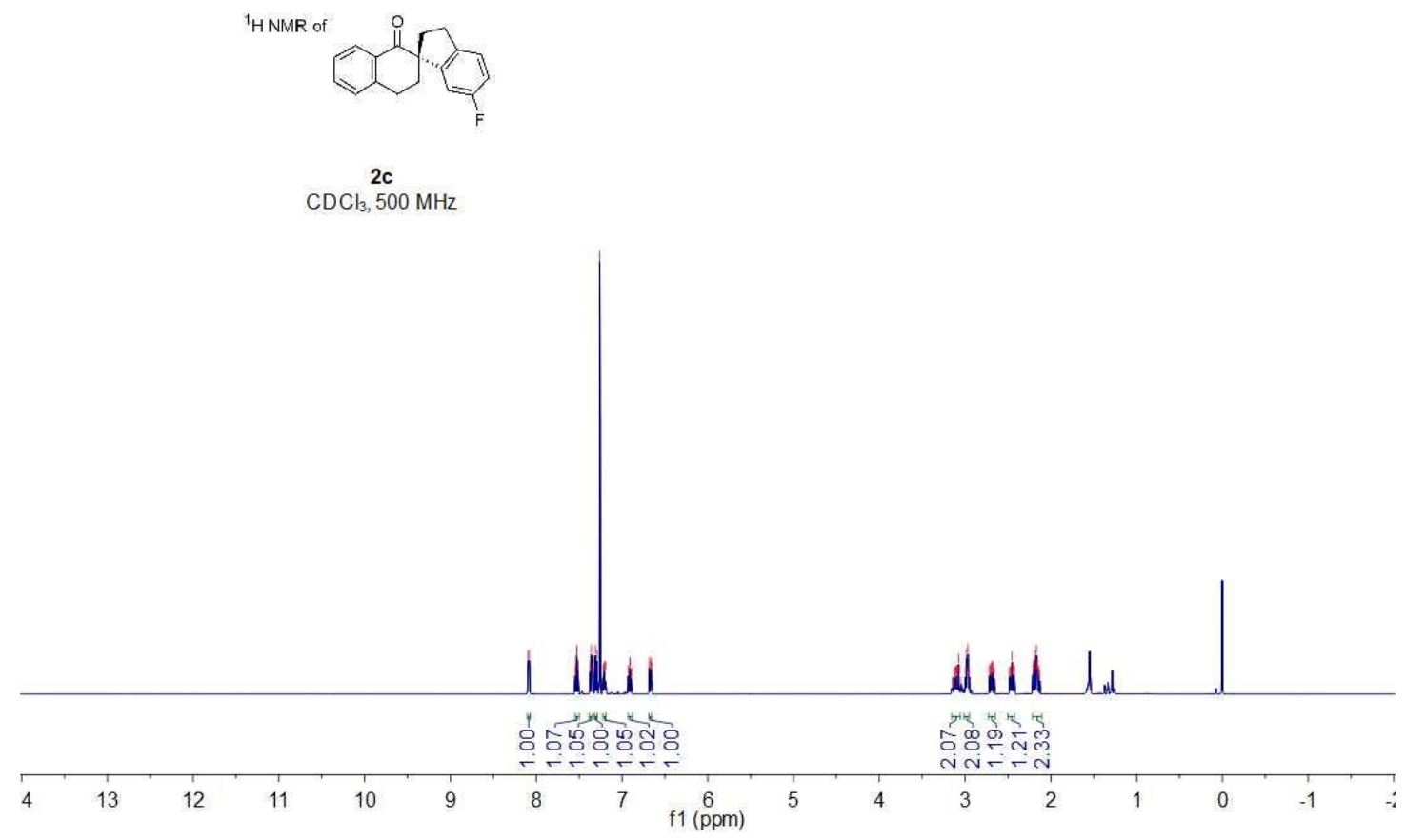

\begin{tabular}{|c|c|c|c|c|}
\hline 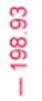 & 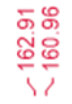 & 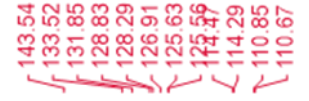 & s. & 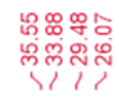 \\
\hline
\end{tabular}

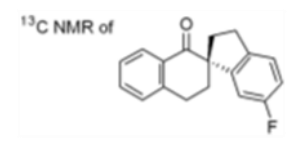

2c

$\mathrm{CDCl}_{3}, 126 \mathrm{MHz}$

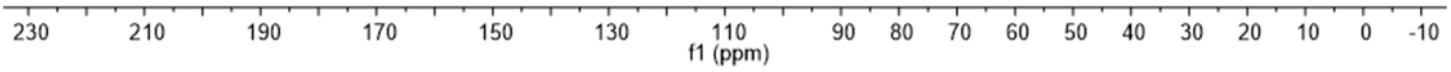



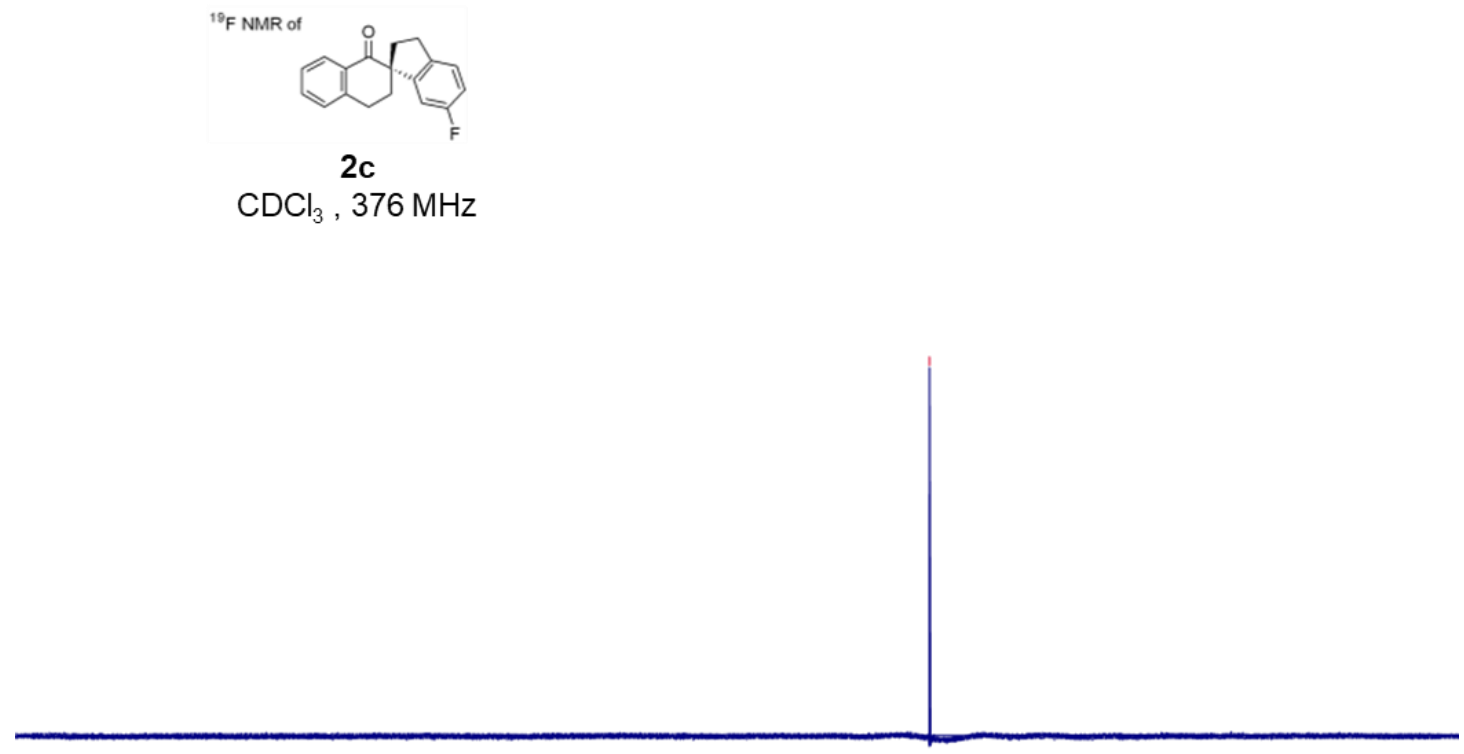

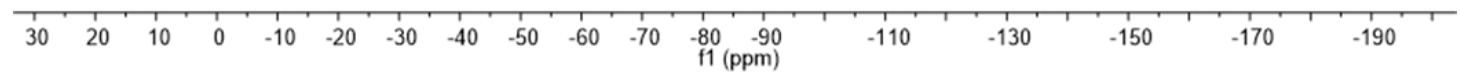

${ }^{1} \mathrm{H}$ NMR of

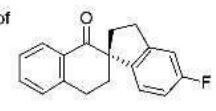

2d $\mathrm{CDCl}_{3}, 500 \mathrm{MHz}$

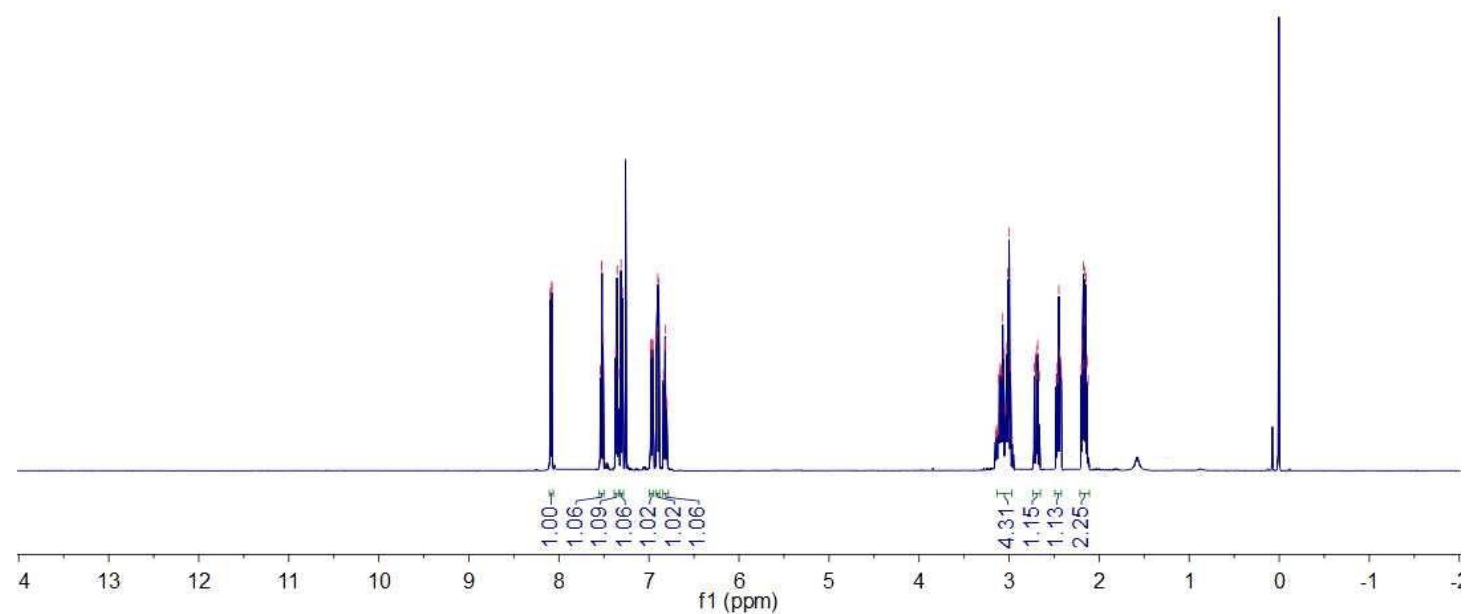



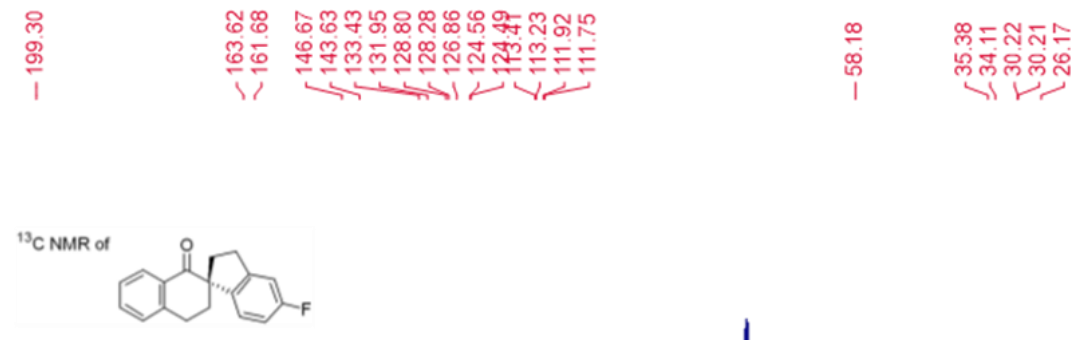

$2 d$

$\mathrm{CDCl}_{3}, 126 \mathrm{MHz}$

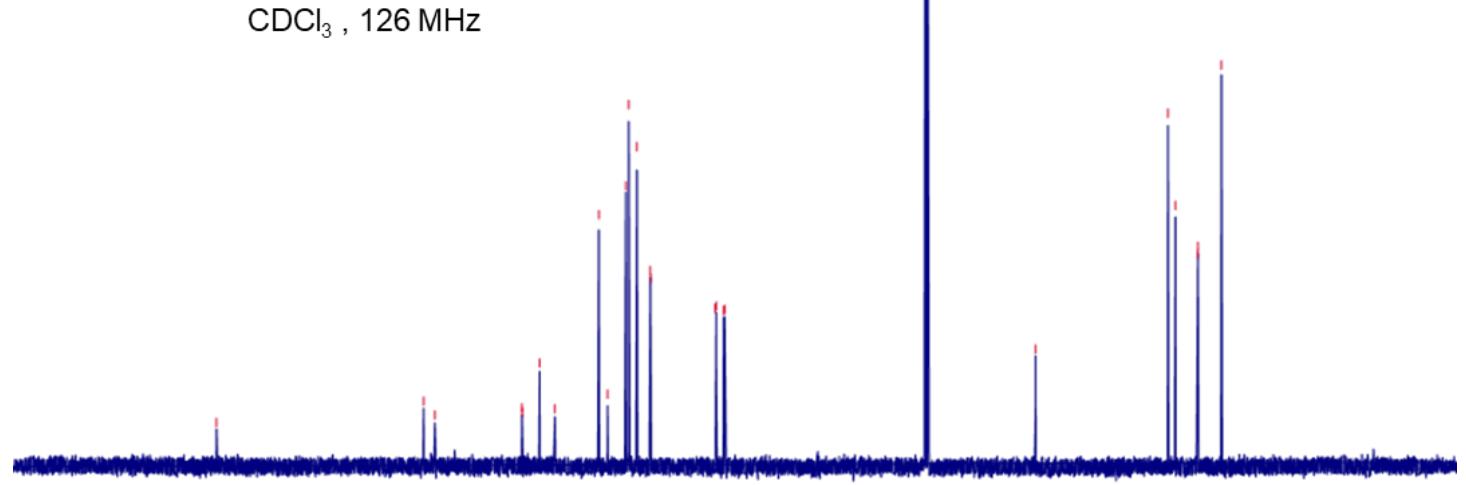

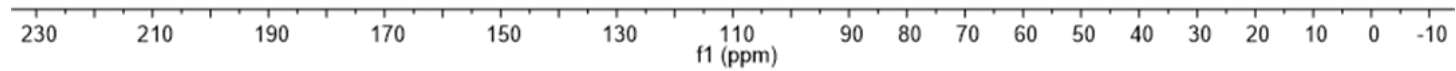

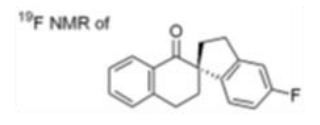

2d

$\mathrm{CDCl}_{3}, 376 \mathrm{MHz}$
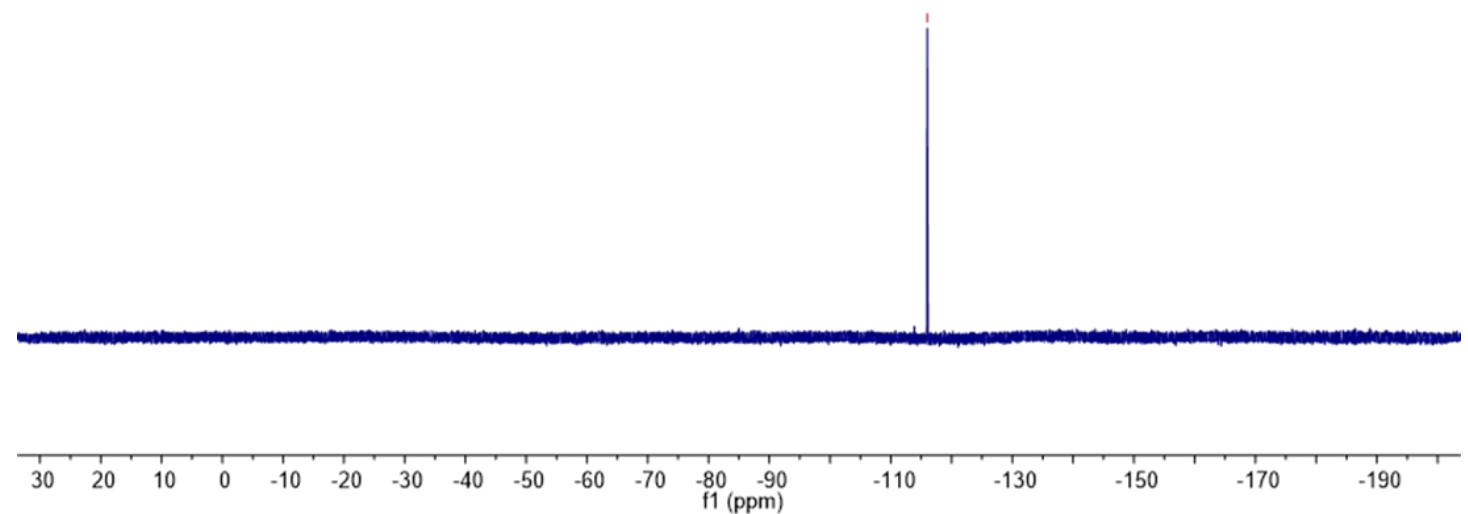


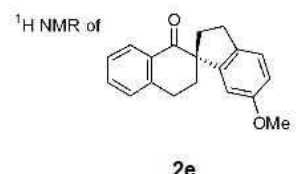

$\stackrel{2 e}{\mathrm{CDCl}_{3}, 500 \mathrm{MHz}}$

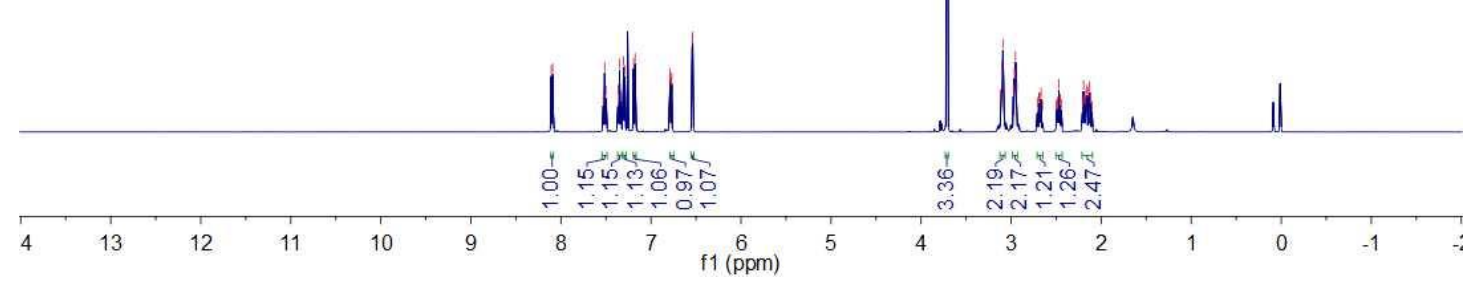

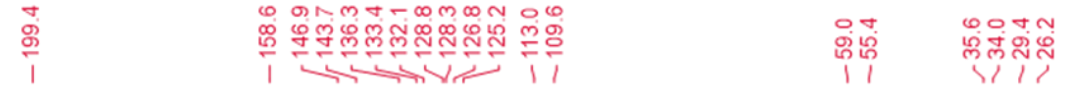

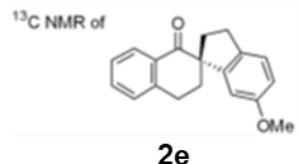

$\mathrm{CDCl}_{3}, 126 \mathrm{MHz}$

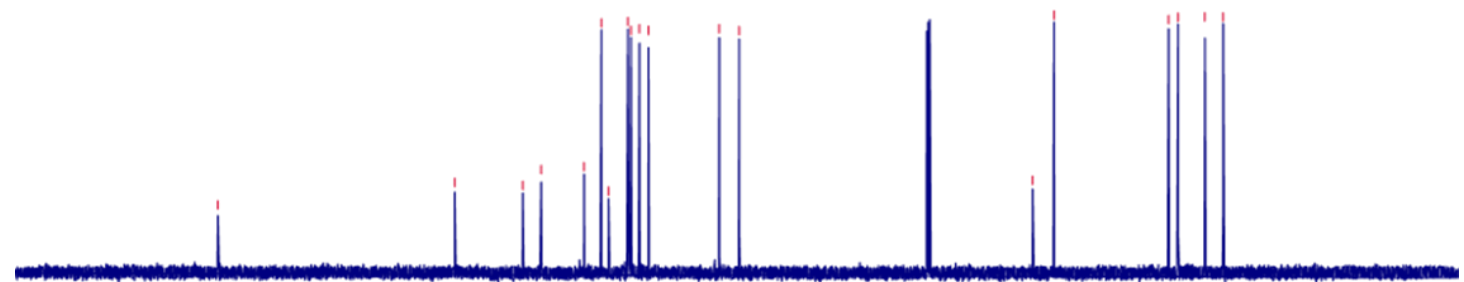

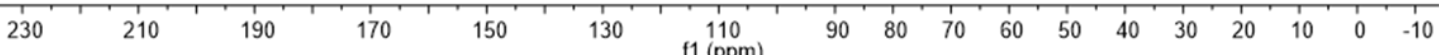


${ }^{1}$ HNMR of

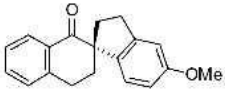

$\stackrel{2 f}{\mathrm{CDCl}_{3}, 500 \mathrm{MHz}}$
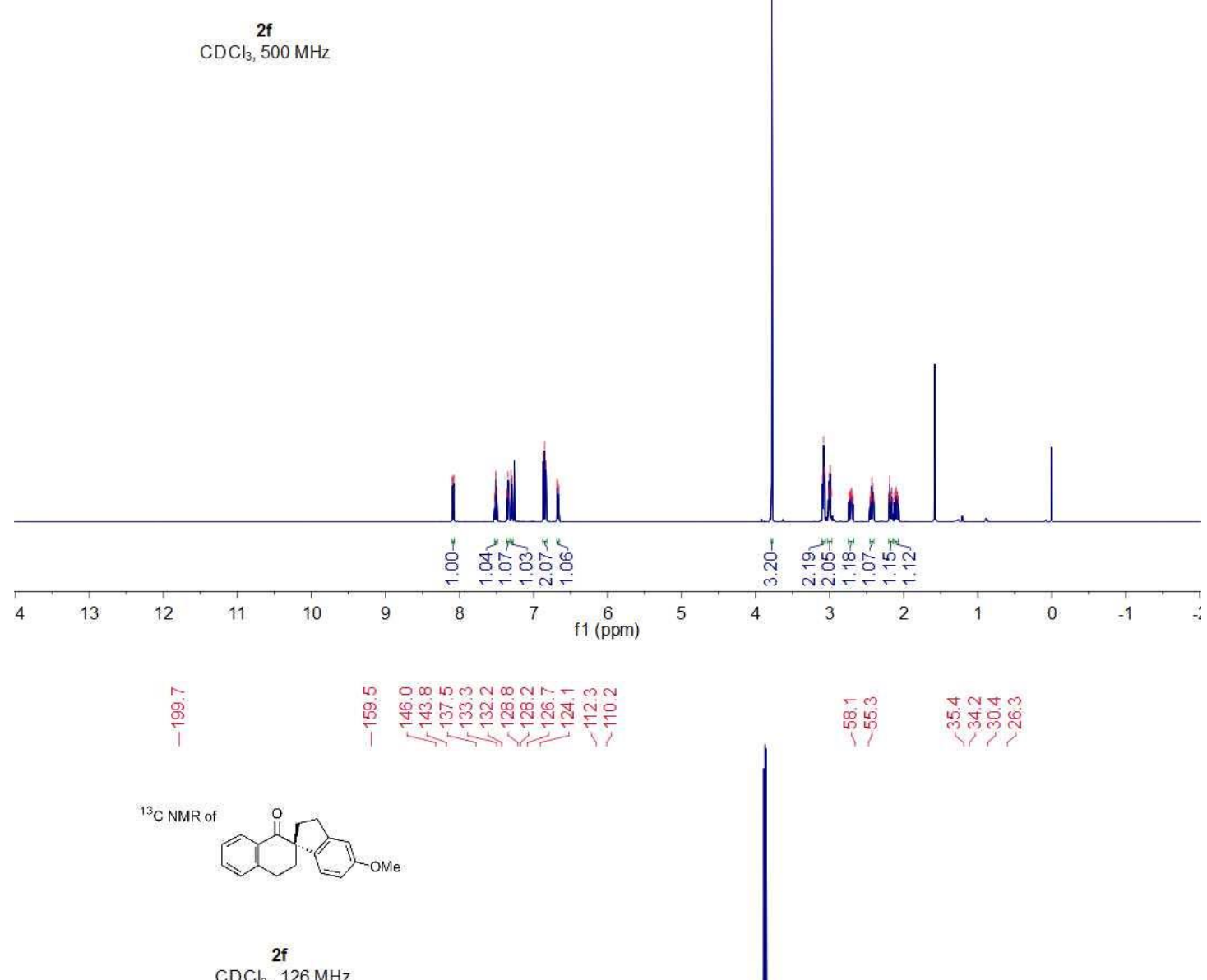

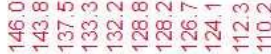

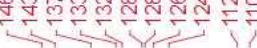

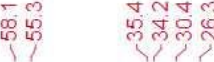

$\mathrm{CDCl}_{3}, 126 \mathrm{MHz}$

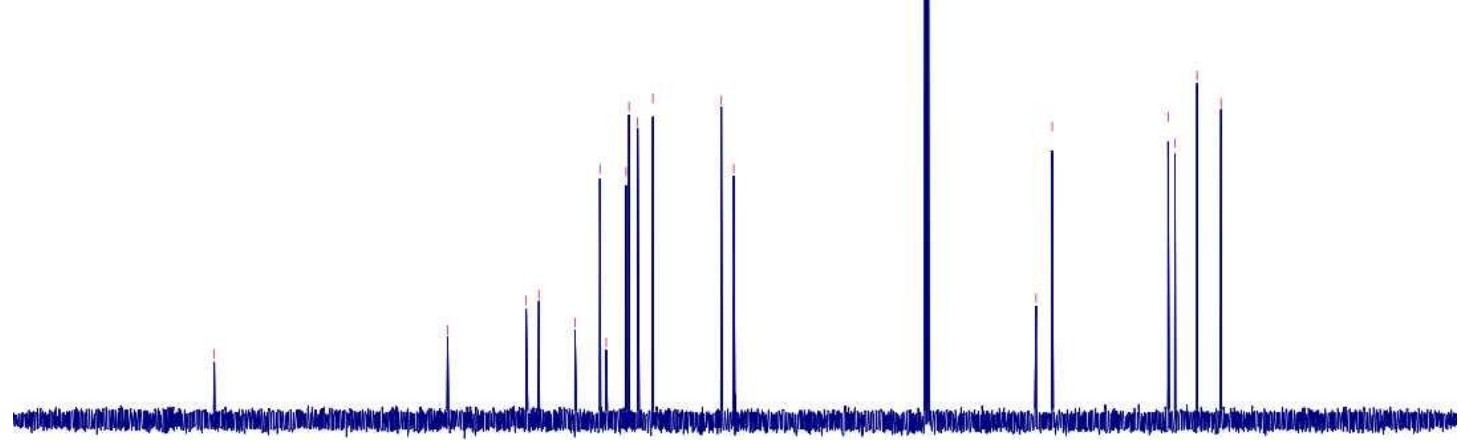

$\begin{array}{lllllllllllllllllllllllllll} & 230 & 210 & 190 & 170 & 150 & 130 & 110 & 90 & 80 & 70 & 60 & 50 & 40 & 30 & 20 & 10 & 0 & -10\end{array}$



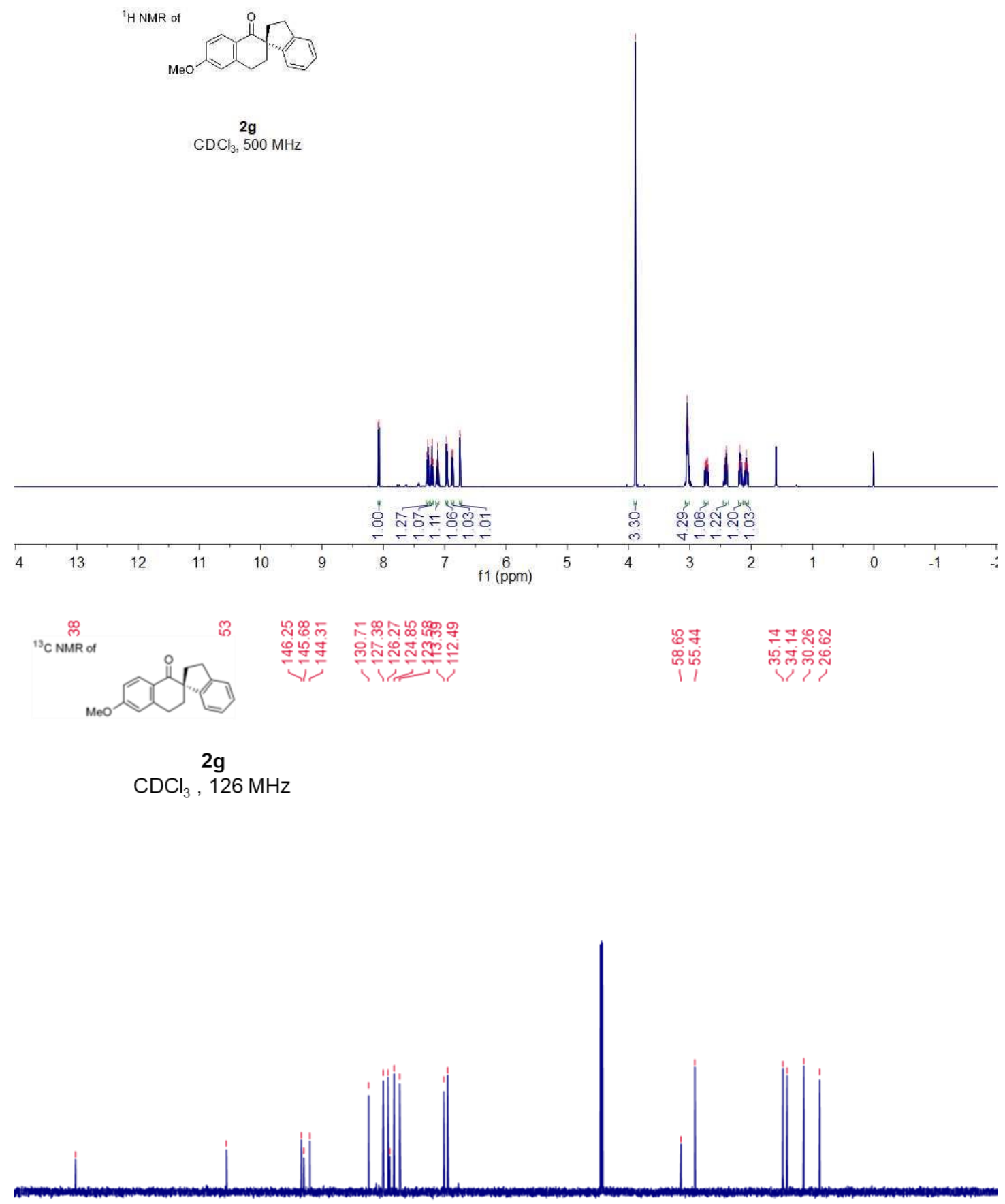

\begin{tabular}{lllllllllllllllllllllllll}
\hline & 110 & 200 & 190 & 180 & 170 & 160 & 150 & 140 & 130 & 120 & 110 & $\begin{array}{c}100 \\
\mathrm{f} 1(\mathrm{ppm})\end{array}$ & 90 & 80 & 70 & 60 & 50 & 40 & 30 & 20 & 10 & 0 & -10
\end{tabular} 


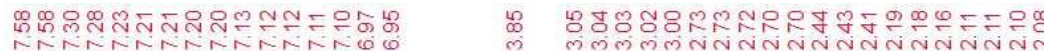

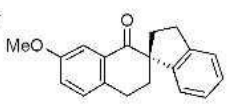

$\stackrel{2 \mathrm{~h}}{\mathrm{CDCl}_{3}, 500 \mathrm{MHz}}$

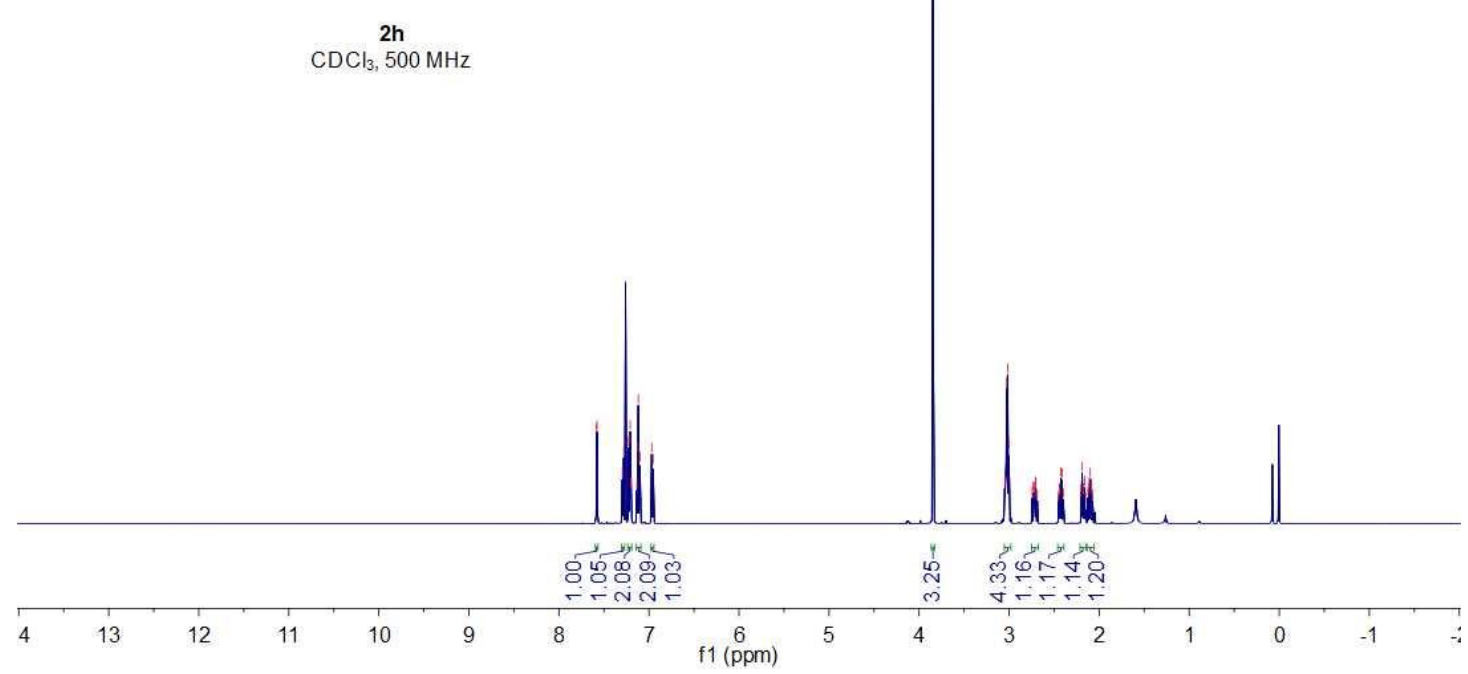

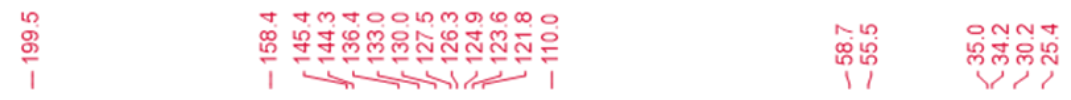

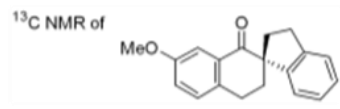

$2 \mathrm{~h}$

$\mathrm{CDCl}_{3}, 126 \mathrm{MHz}$

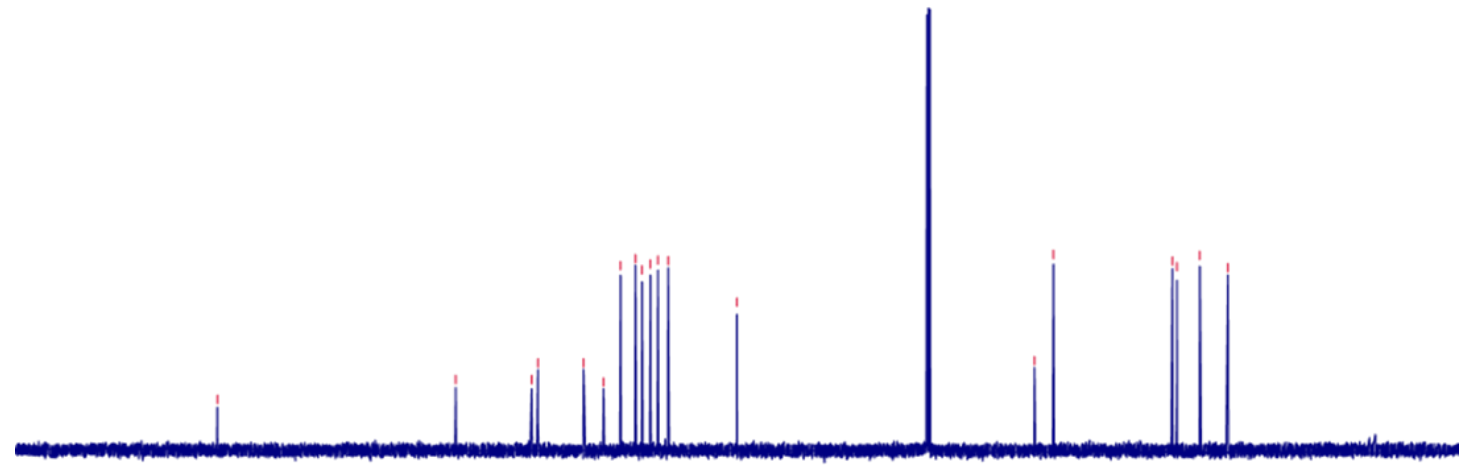

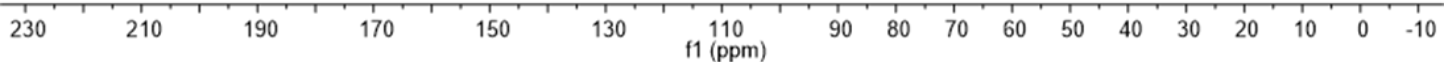


${ }^{1}$ HNMR of

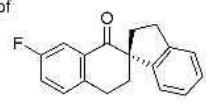

2

$\mathrm{CDCl}_{3}, 500 \mathrm{MHz}$

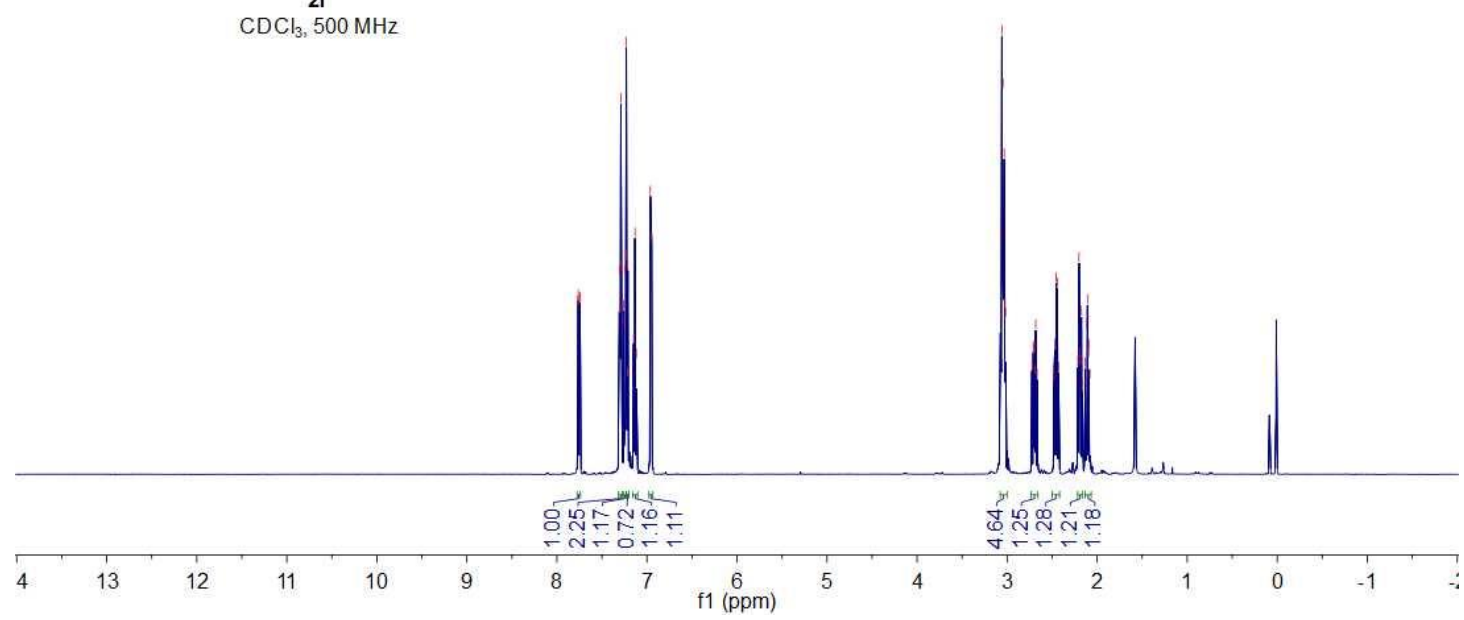

\begin{tabular}{l} 
กิ \\
$\infty$ \\
\multirow{\infty}{\sigma}{}
\end{tabular}

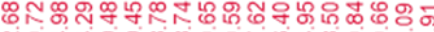

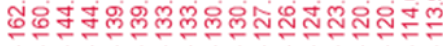

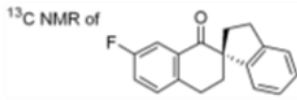

$2 \mathrm{i}$

$\mathrm{CDCl}_{3}, 126 \mathrm{MHz}$

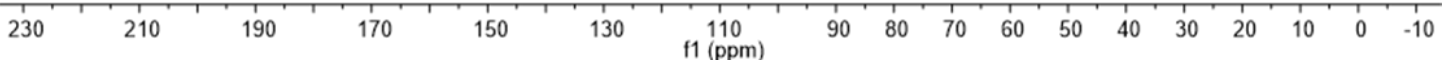




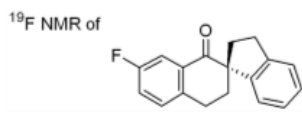

$2 \mathbf{i}$

$\mathrm{CDCl}_{3}, 376 \mathrm{MHz}$
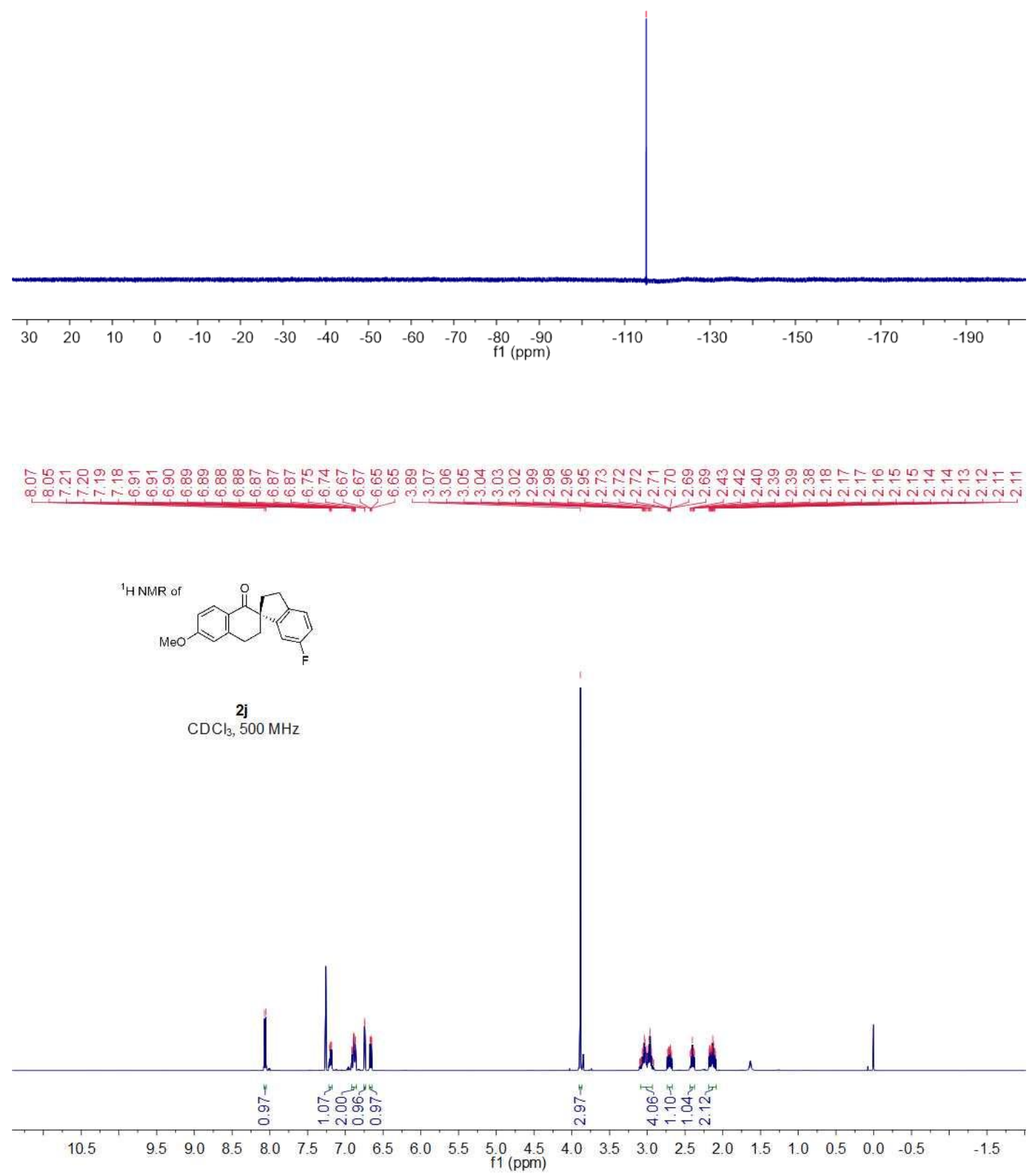


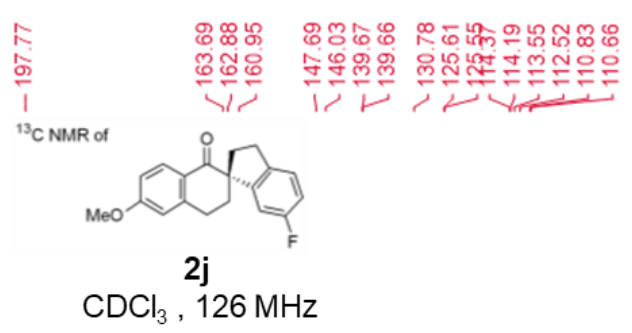

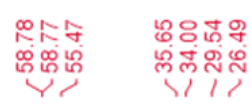
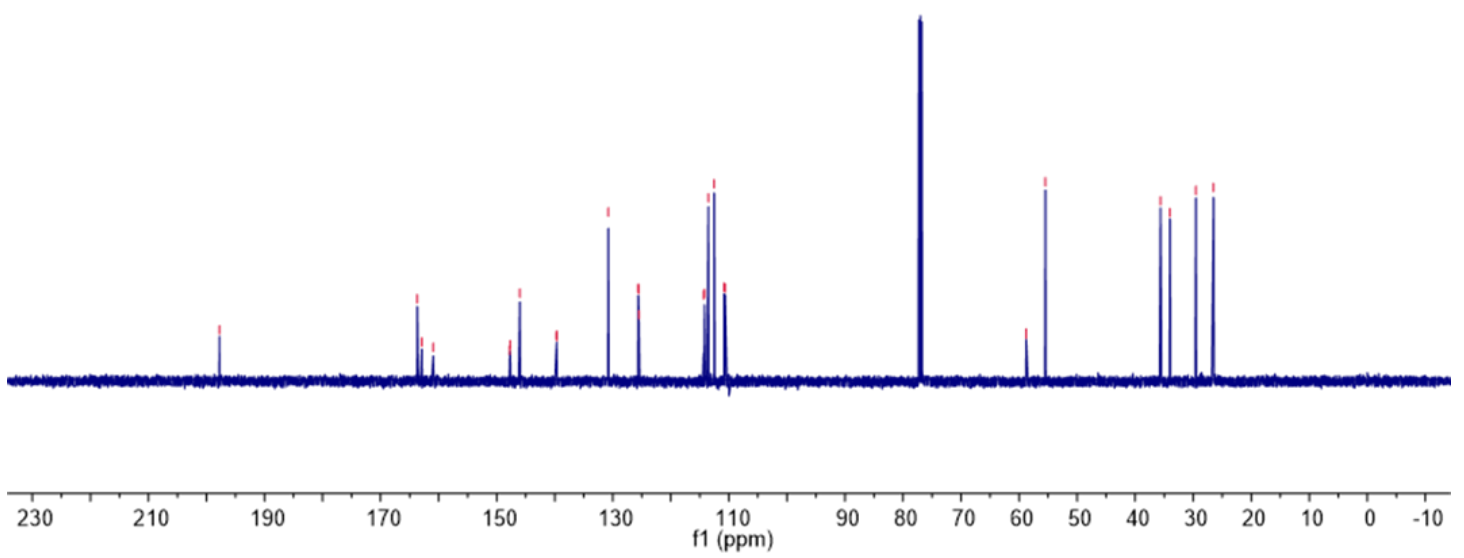

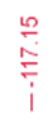
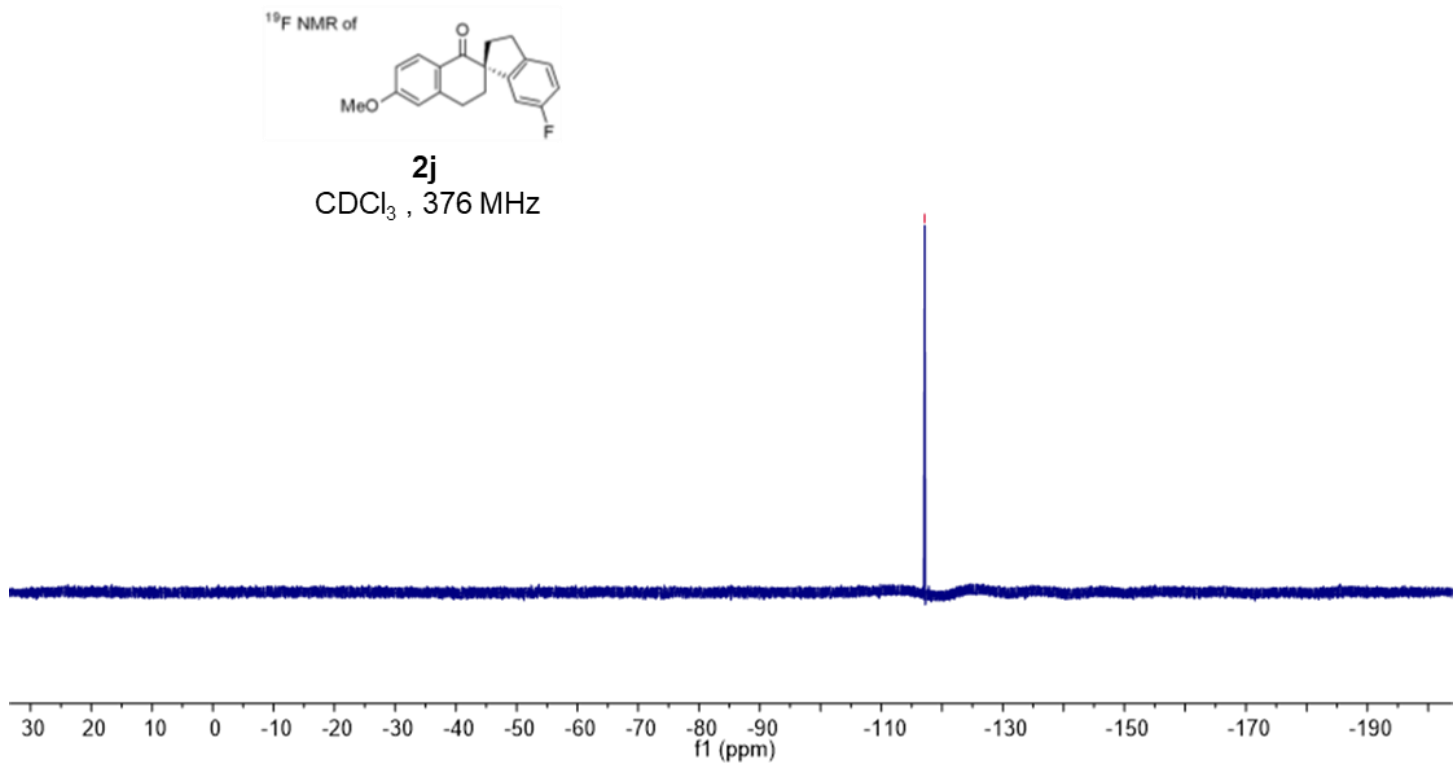

108 
${ }^{1}$ HNMR of

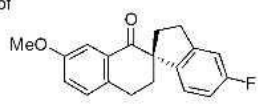

$\mathrm{CDCl}_{3}, 500 \mathrm{MHz}$
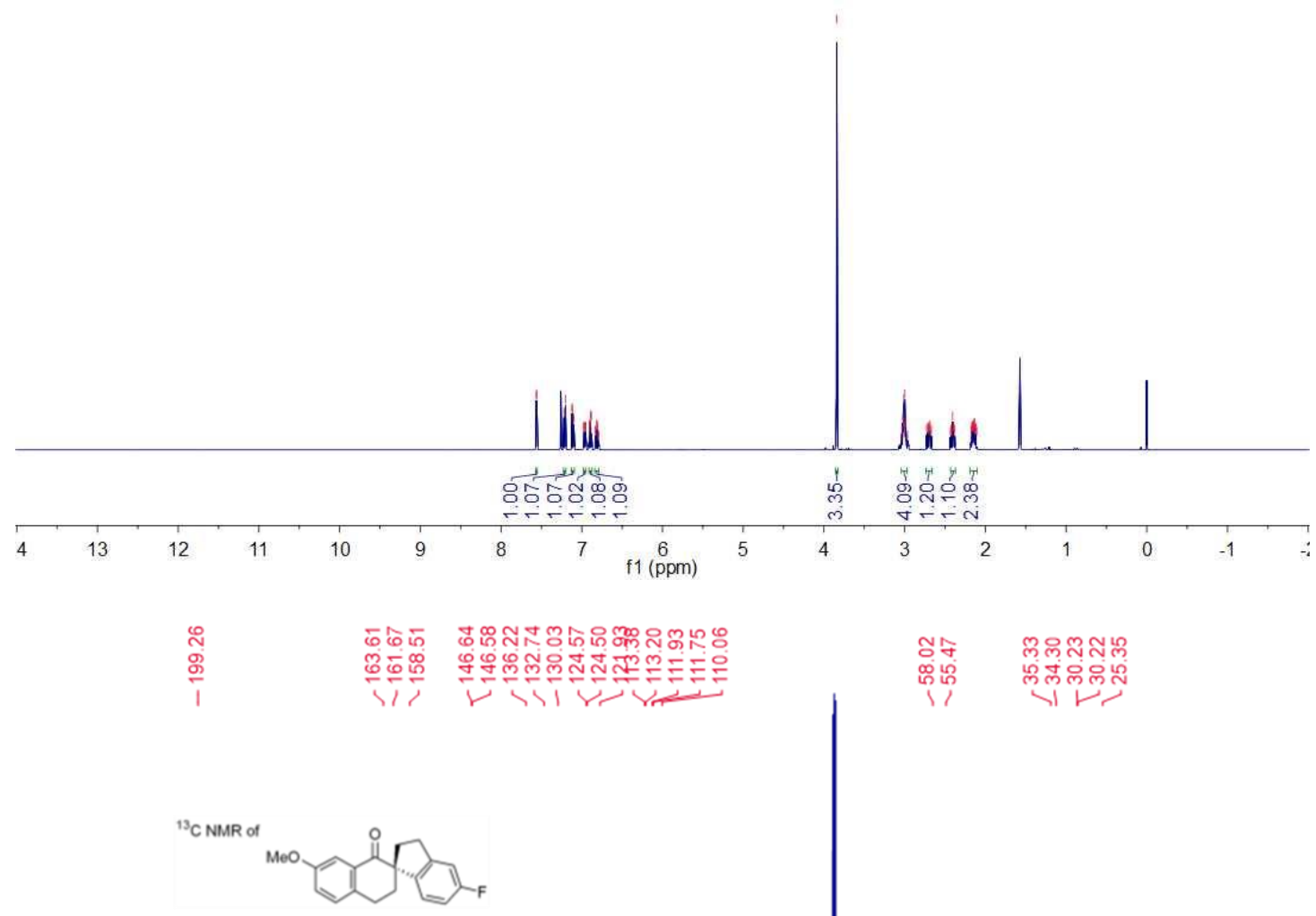

$2 \mathbf{k}$

$\mathrm{CDCl}_{3}, 126 \mathrm{MHz}$

190

170

150

30

$\stackrel{110}{f 1(\mathrm{ppm})}$

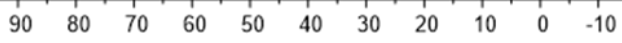



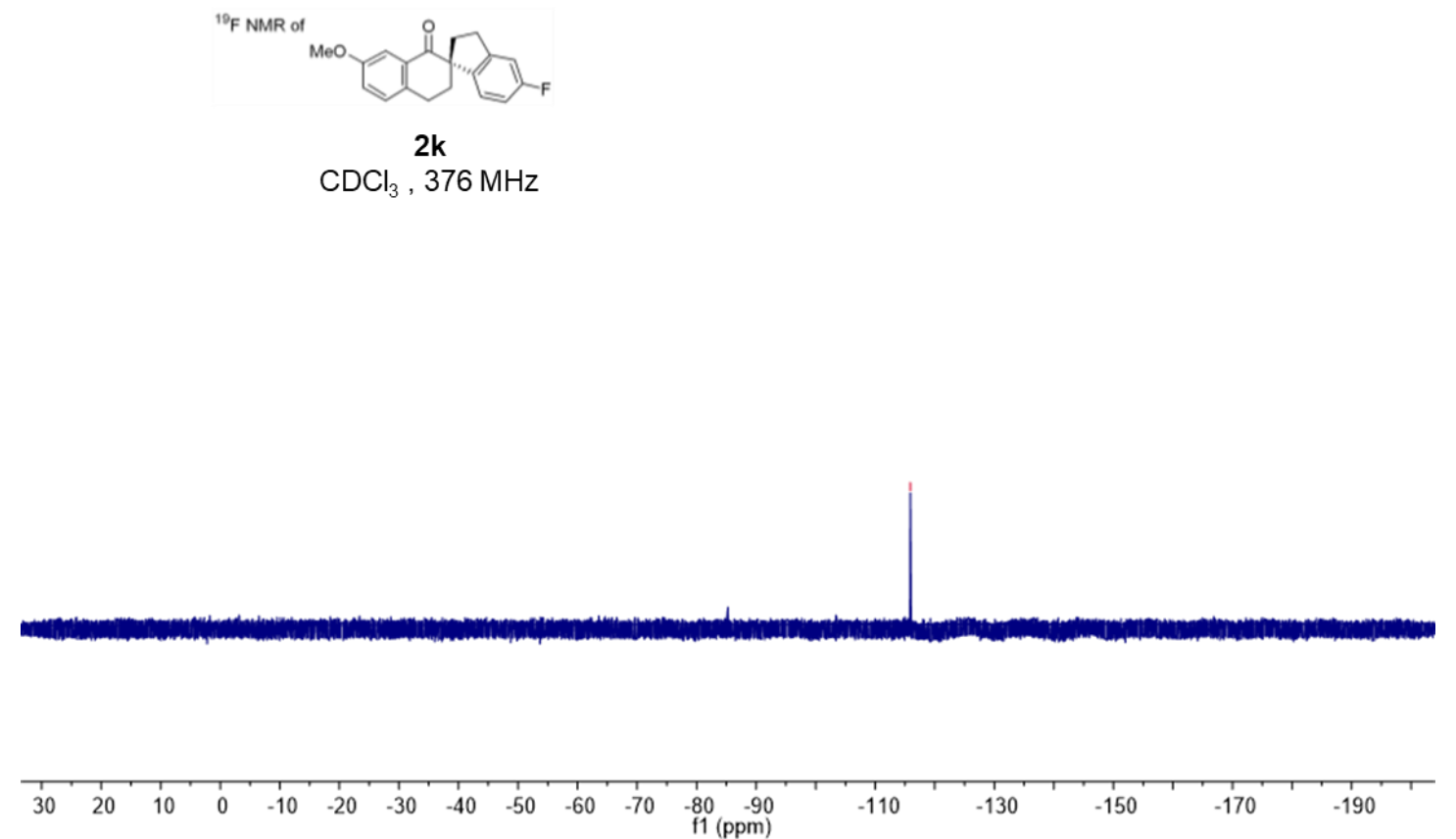

${ }^{1} \mathrm{H}$ NMR of

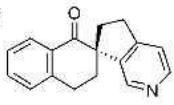

$\stackrel{21}{\mathrm{CDCl}_{3}, 500 \mathrm{MHz}}$

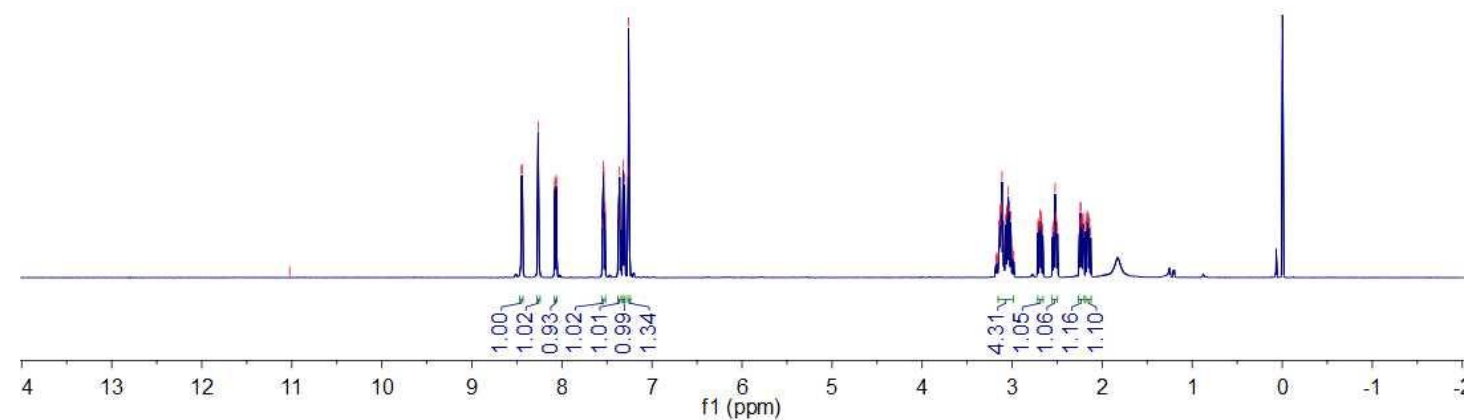



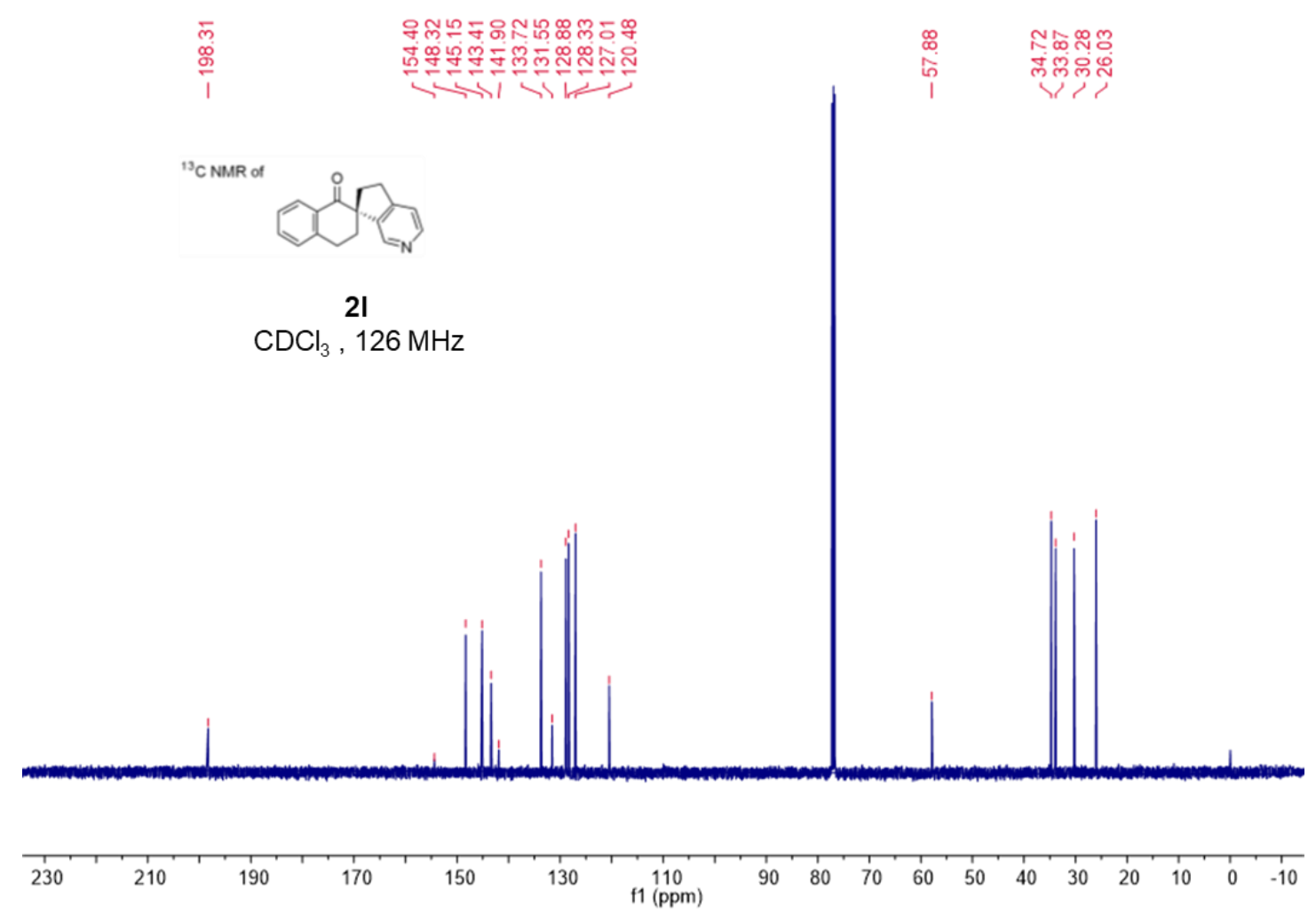

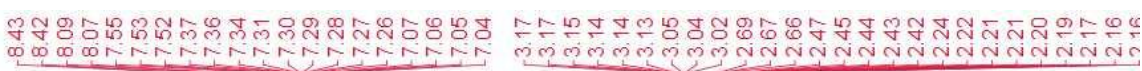

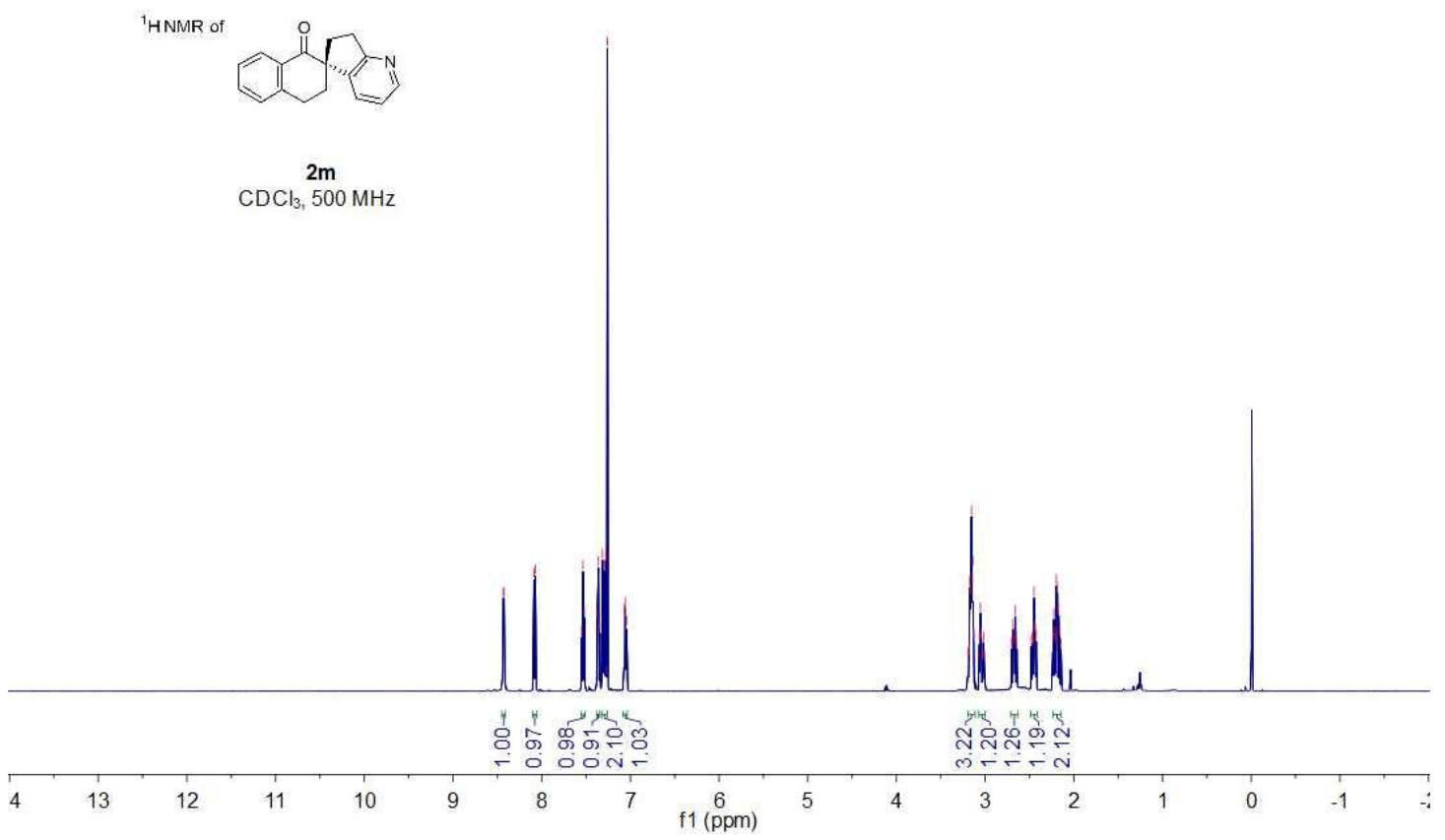

111 


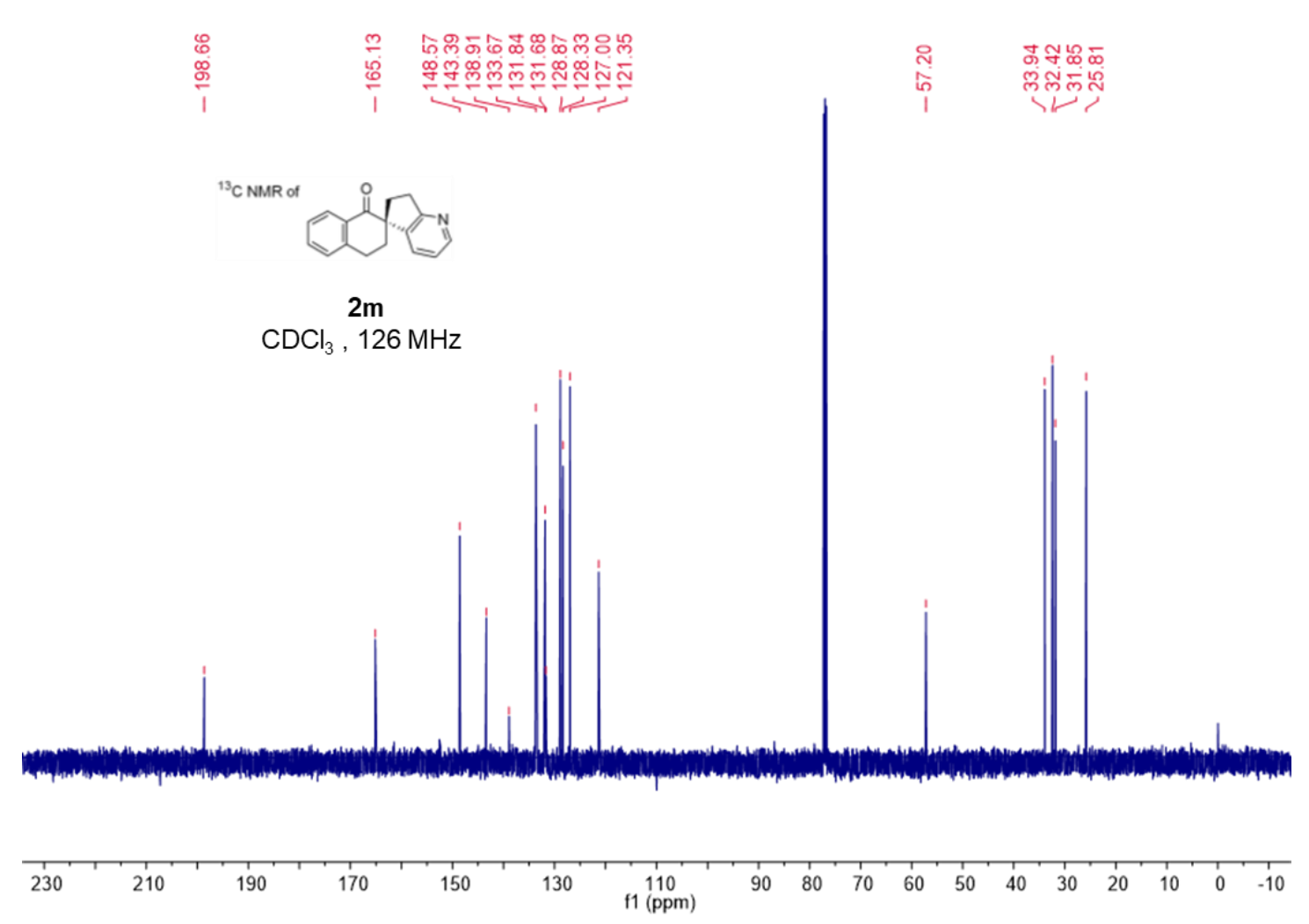

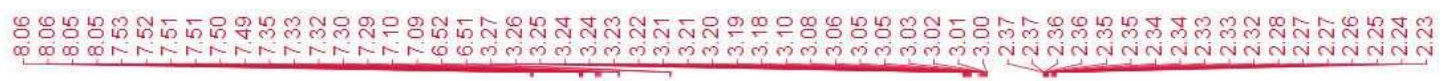

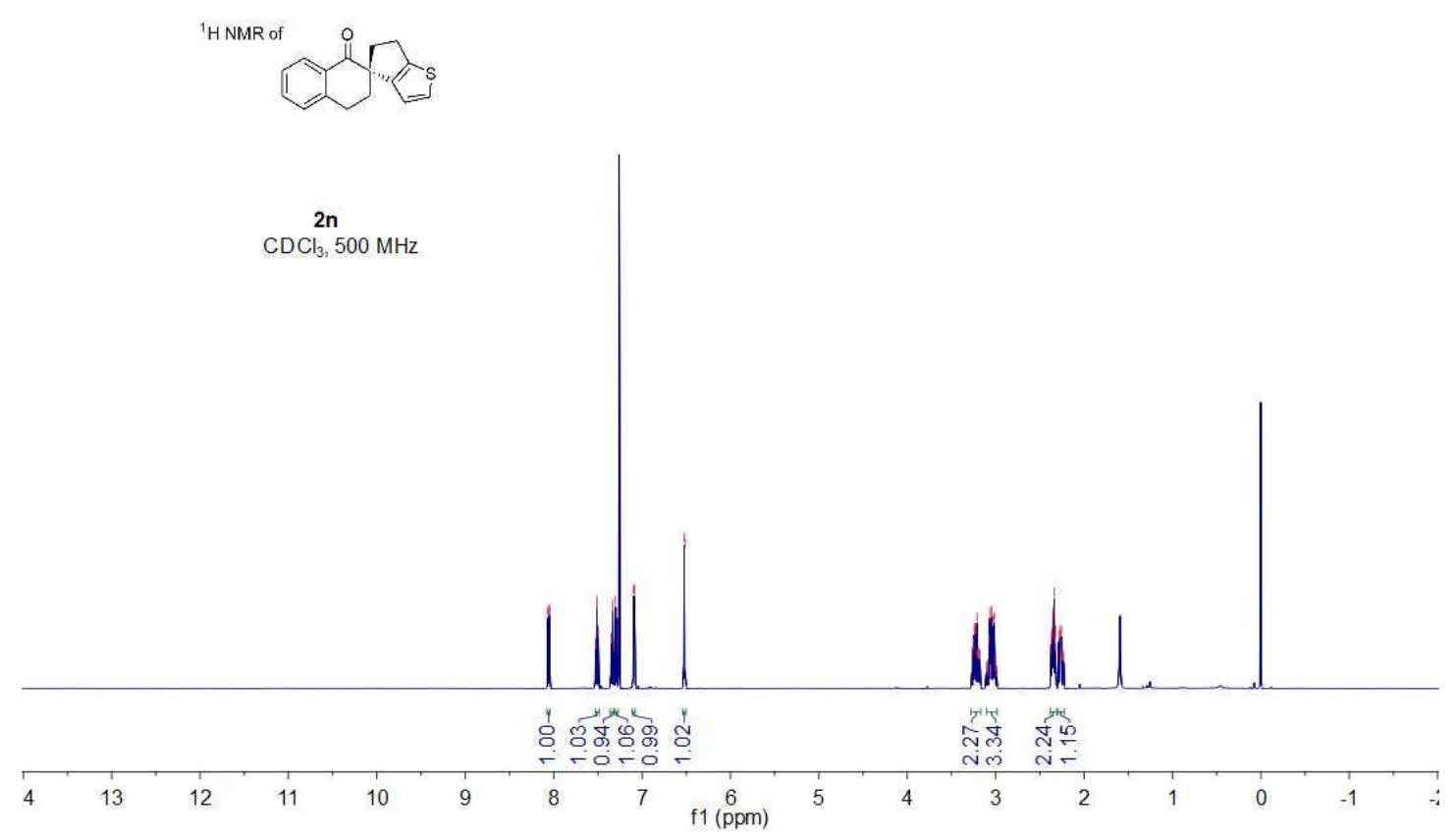

112 

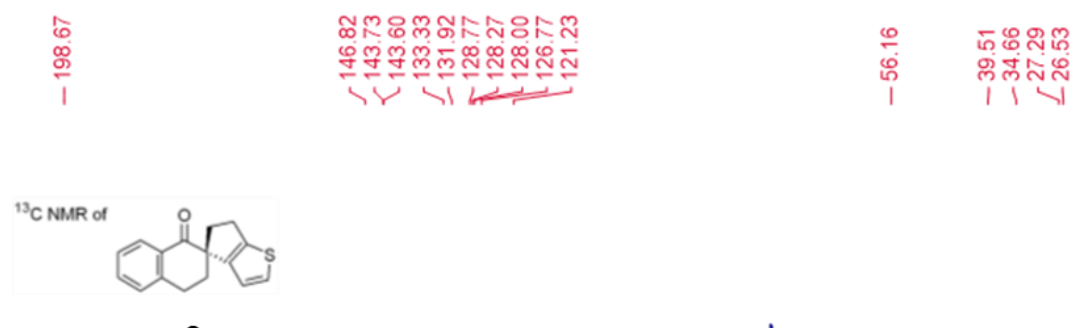

2n

$\mathrm{CDCl}_{3}, 126 \mathrm{MHz}$

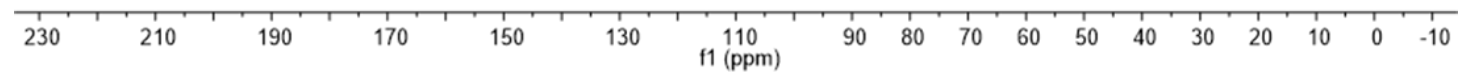
môn

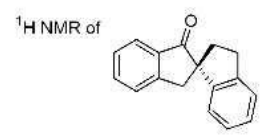

$\stackrel{20}{\mathrm{CDCl}_{3}, 500 \mathrm{MHz}}$

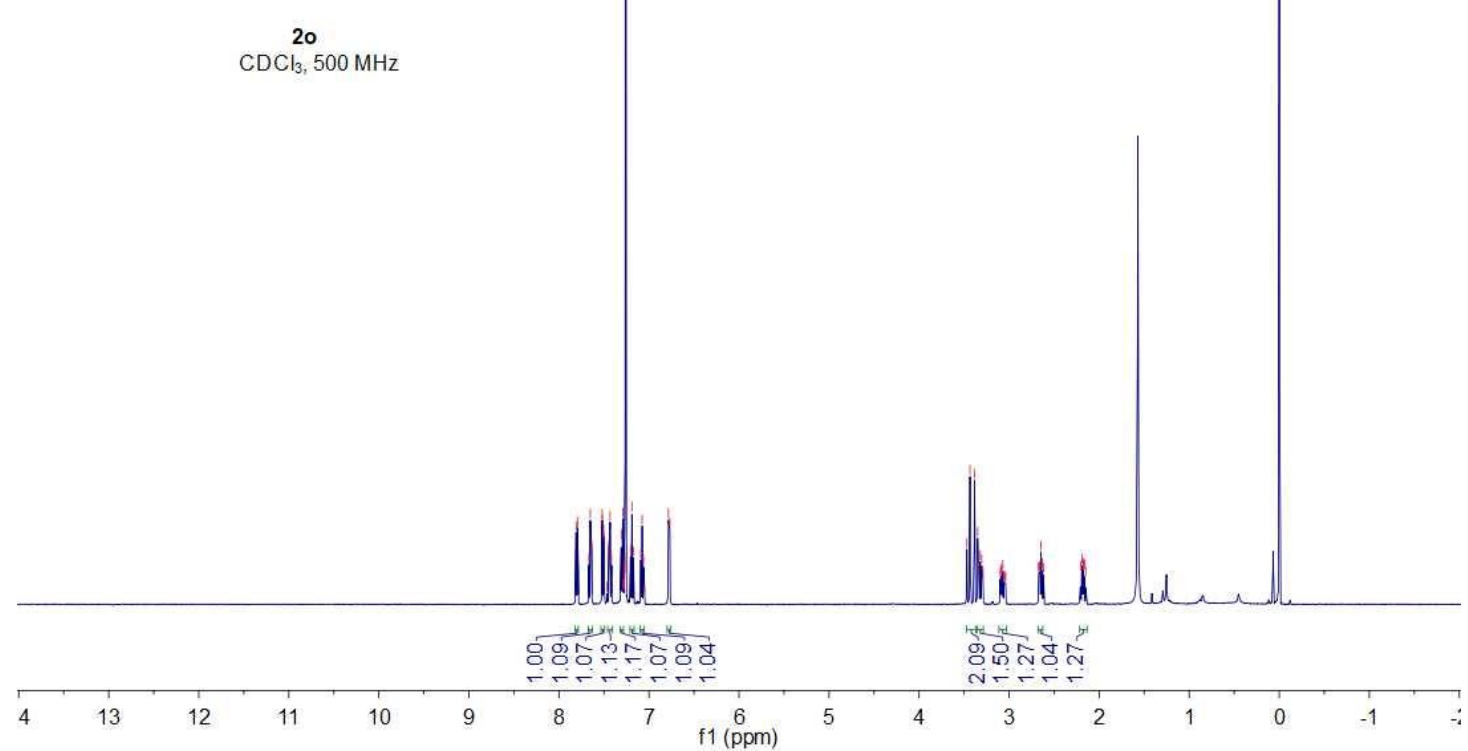


ำ

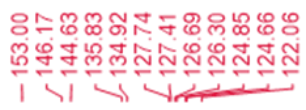

œ

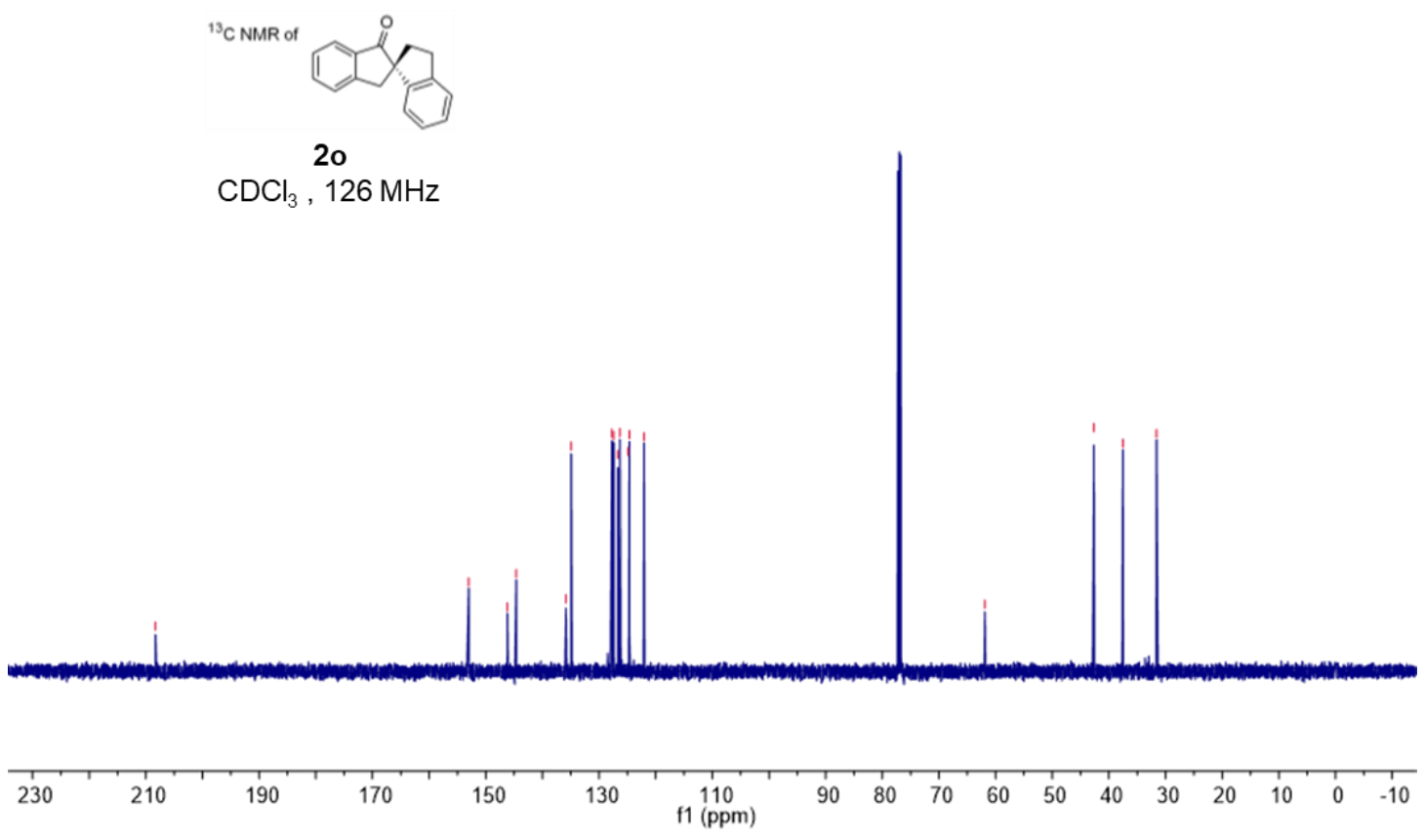

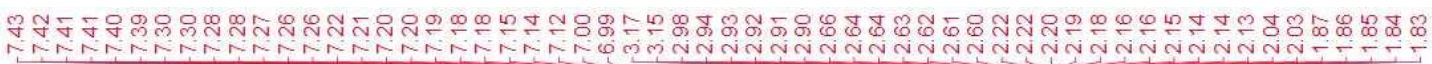

${ }^{1} \mathrm{H}$ NMR of

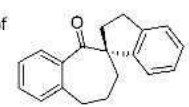

$\stackrel{2 p}{2 p} \mathrm{CDCl}_{3}, 500 \mathrm{MHz}$

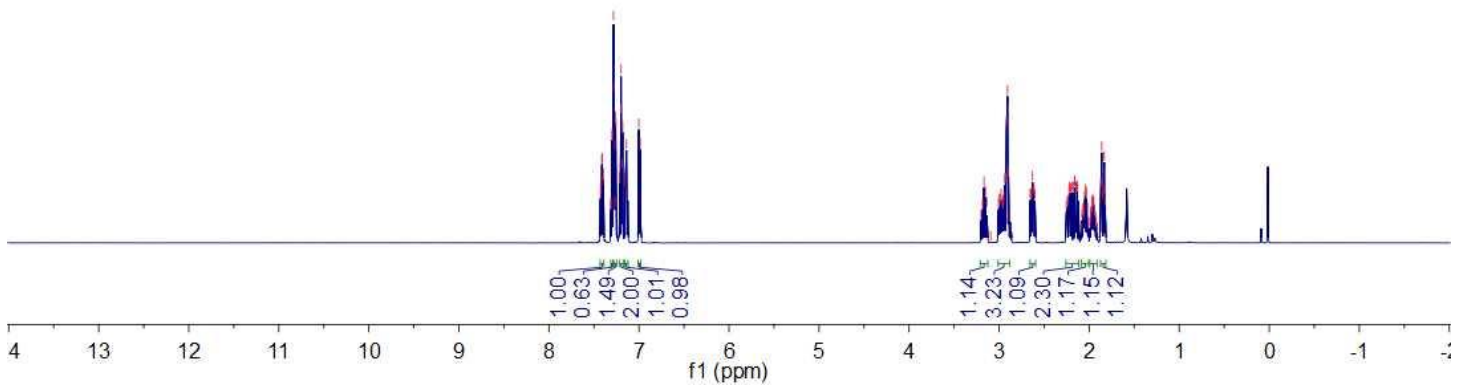

114 

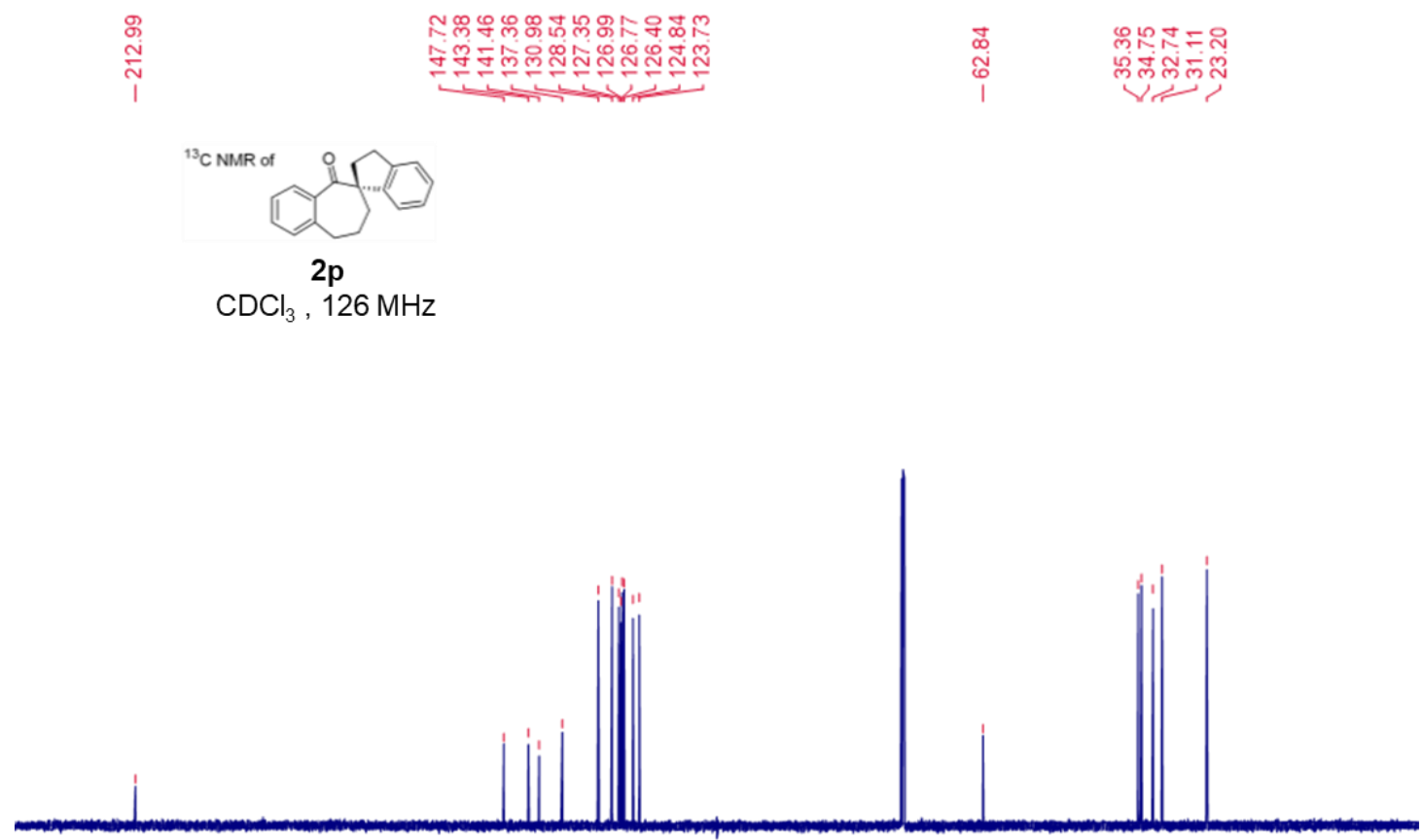

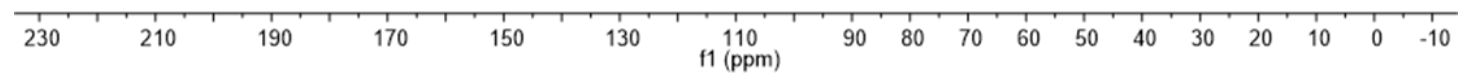

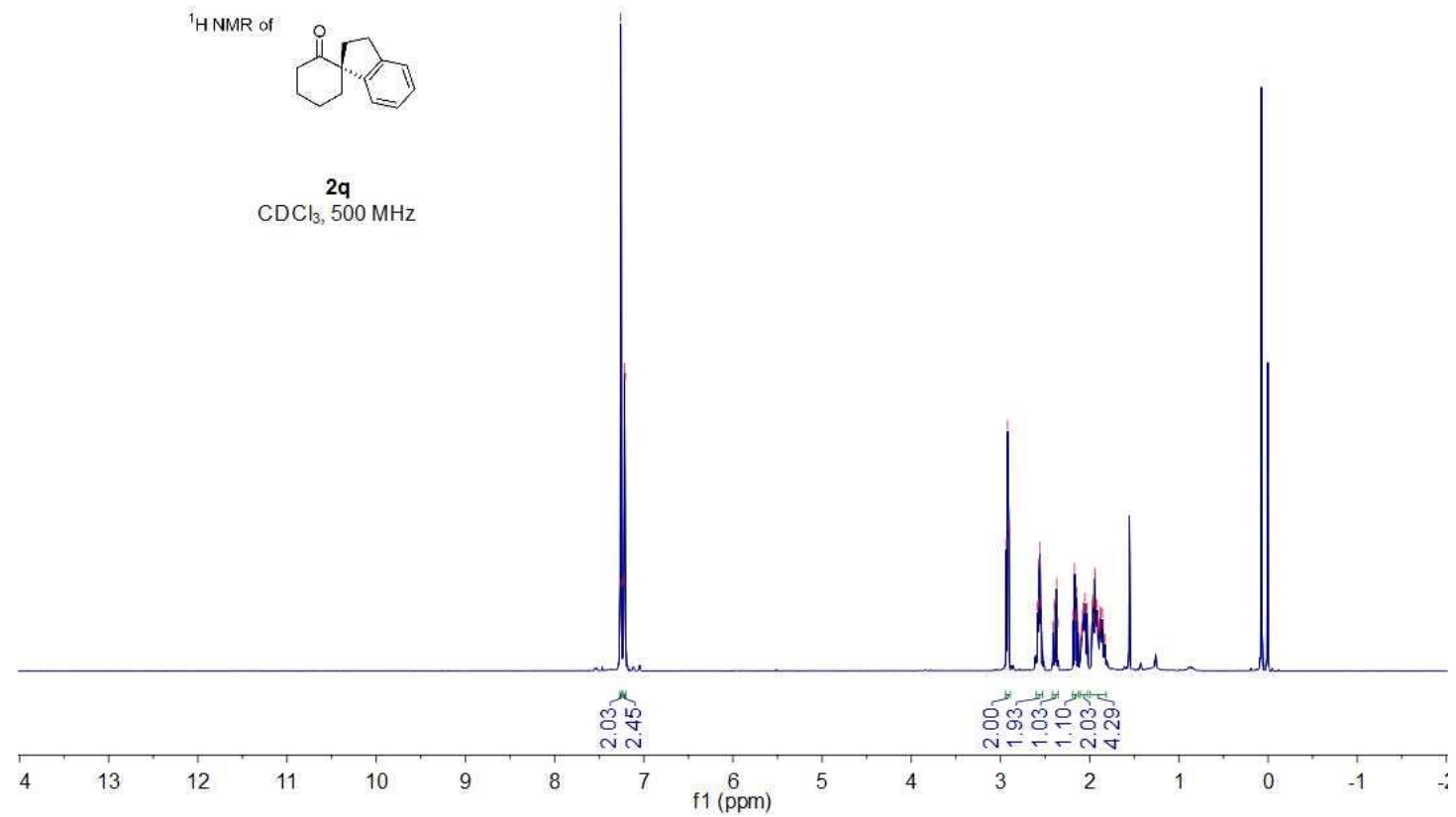




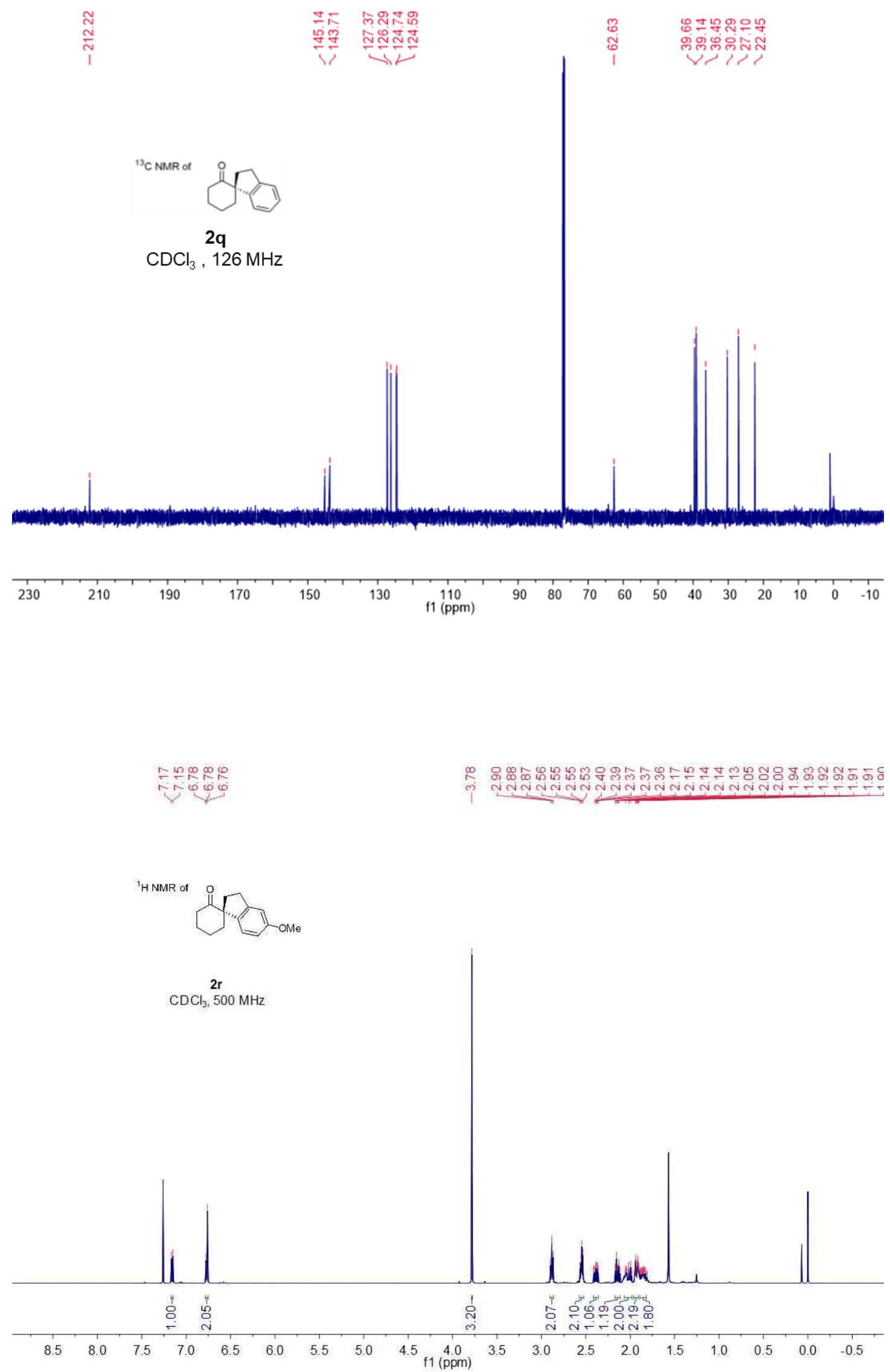



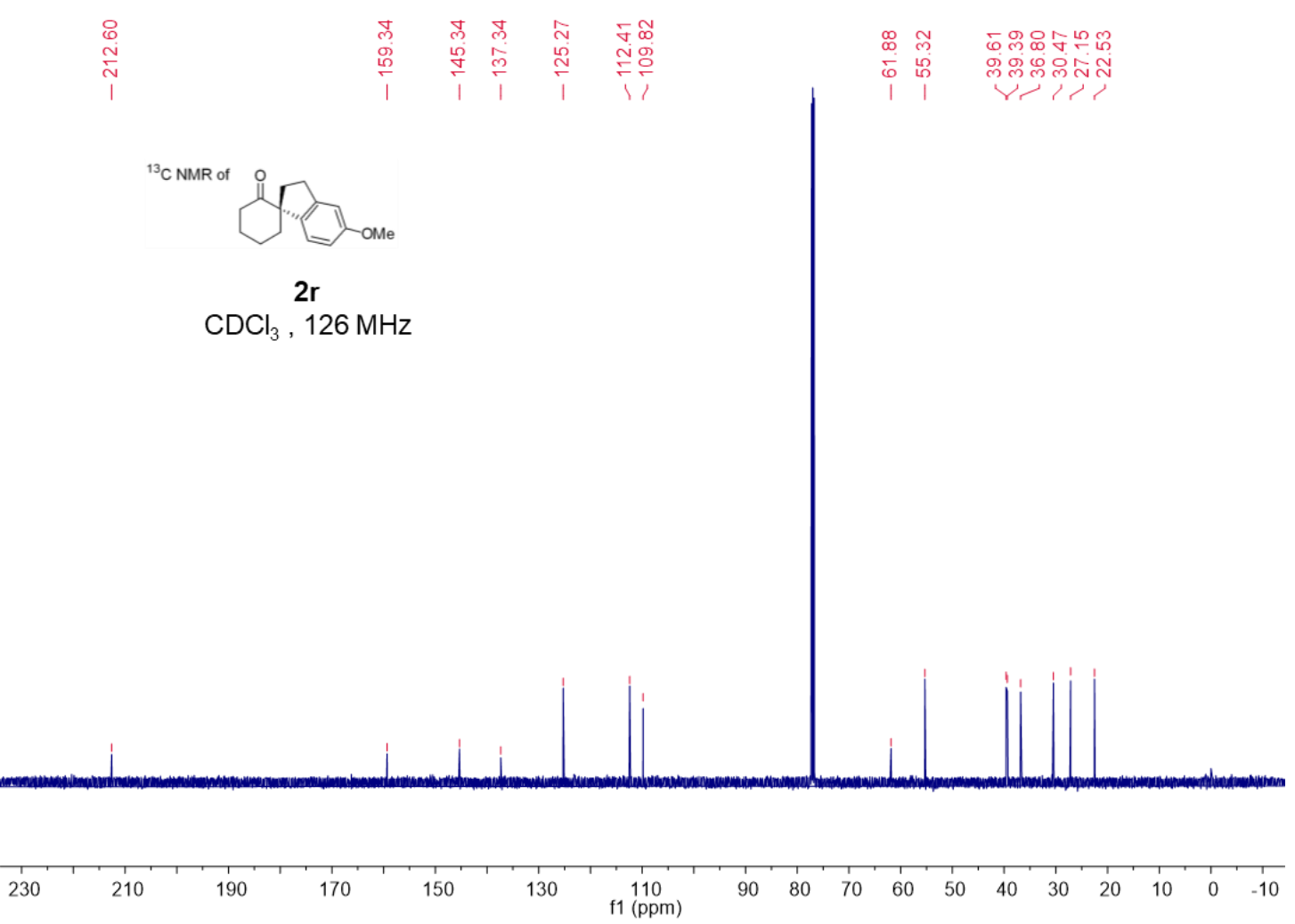

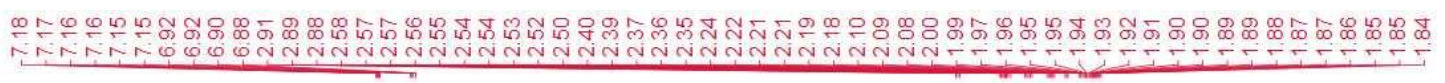

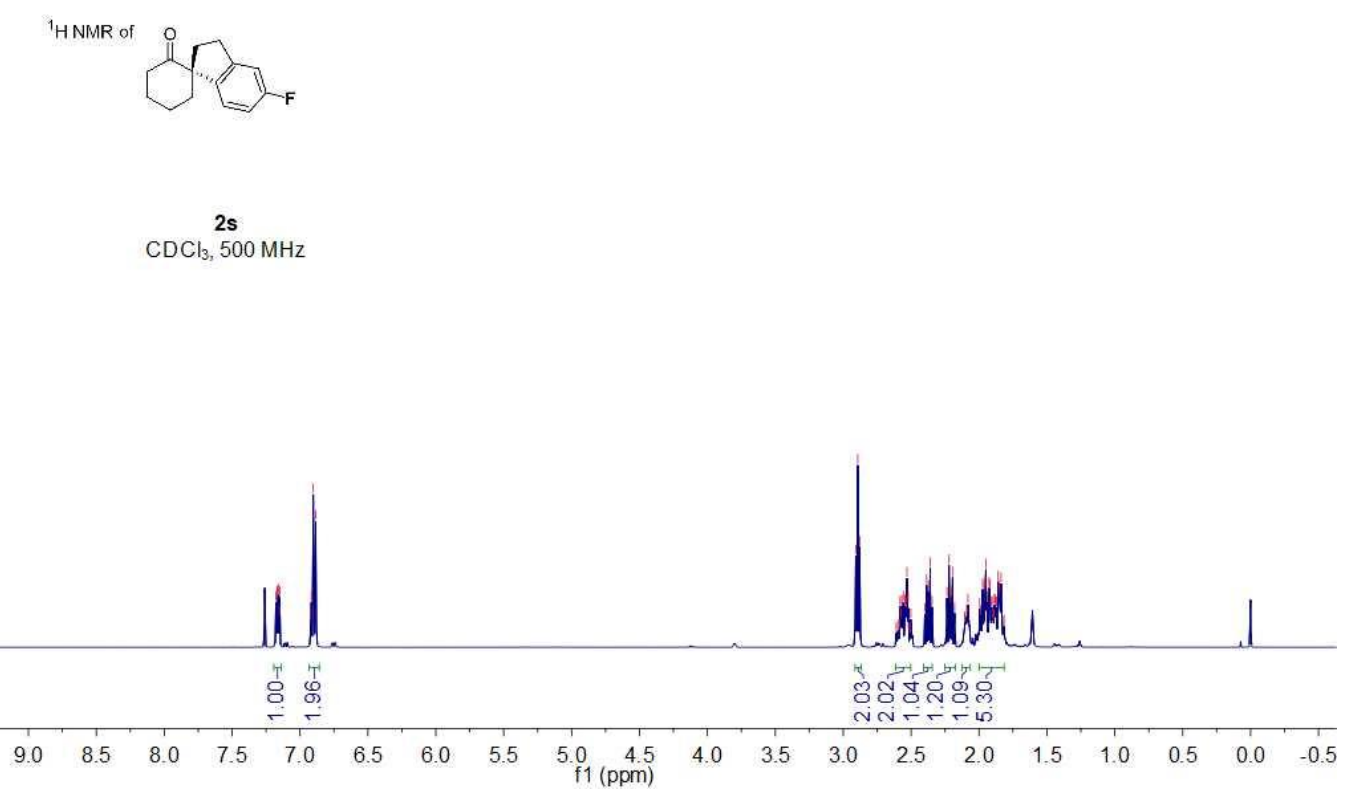

117 
$\stackrel{\frac{2}{2}}{\stackrel{1}{N}}$

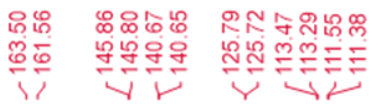

\%

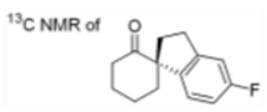

2s

$\mathrm{CDCl}_{3}, 126 \mathrm{MHz}$
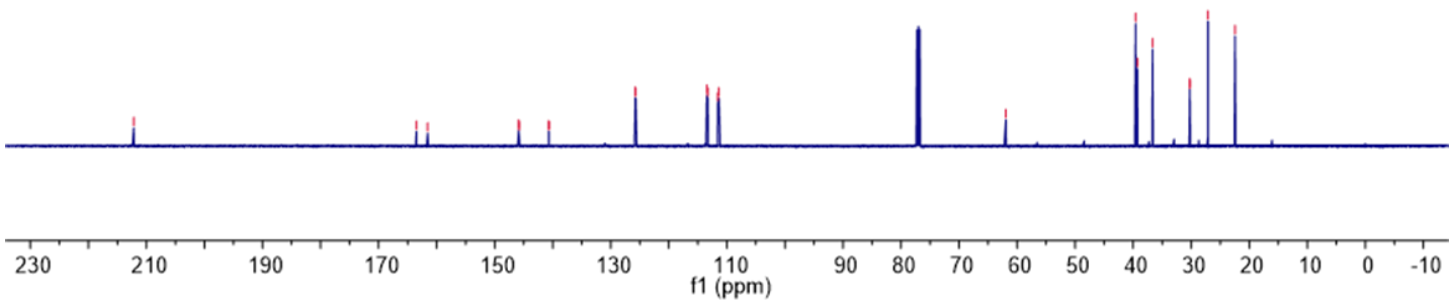

$\underset{i}{\stackrel{i}{i}}$
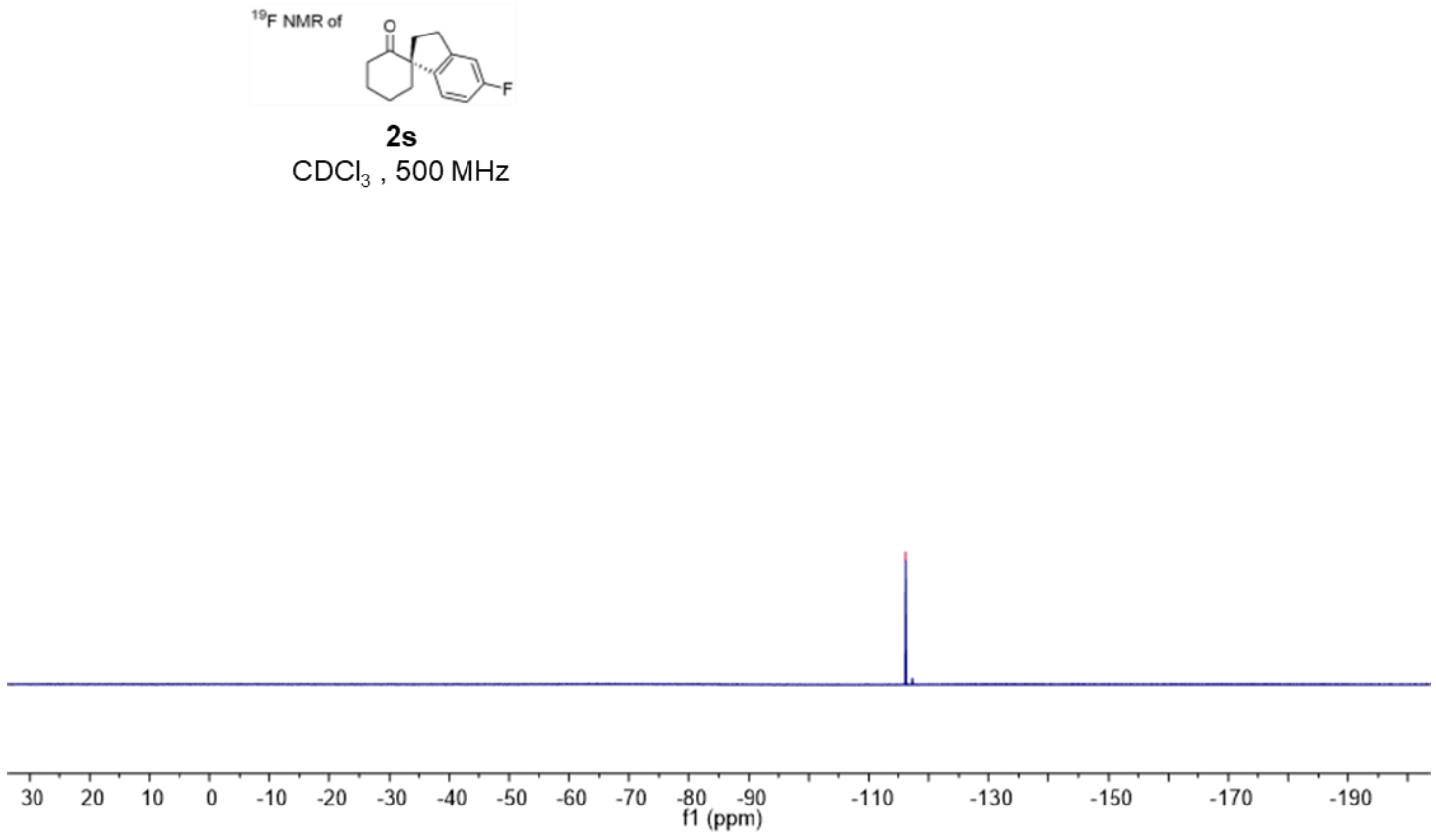

118 
1HNMR of

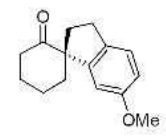

2t

$\mathrm{CDCl}_{3}, 500 \mathrm{MHz}$
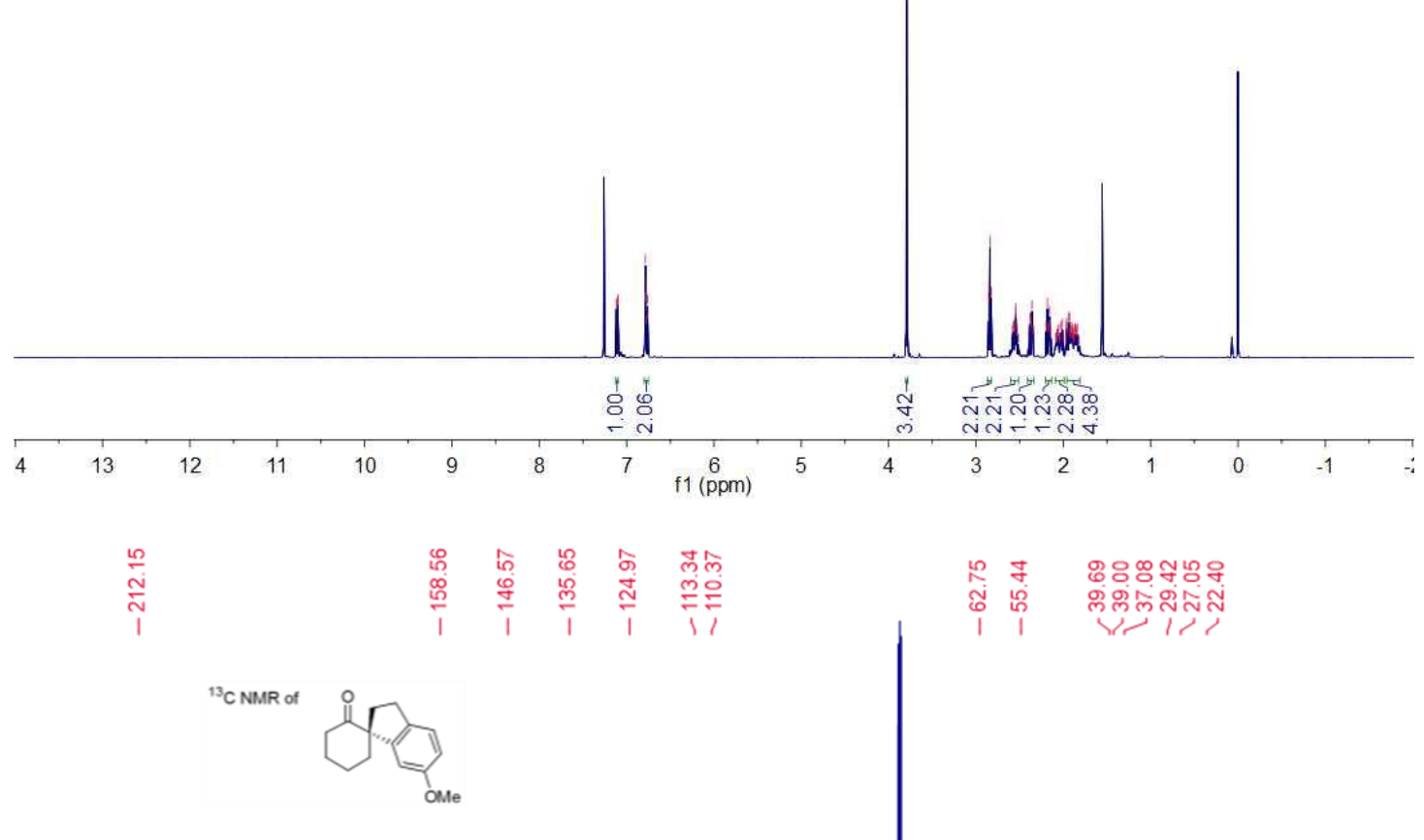

$2 \mathrm{t}$

$\mathrm{CDCl}_{3}, 126 \mathrm{MHz}$

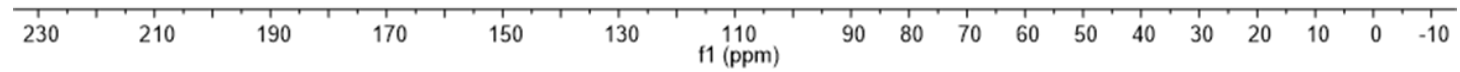



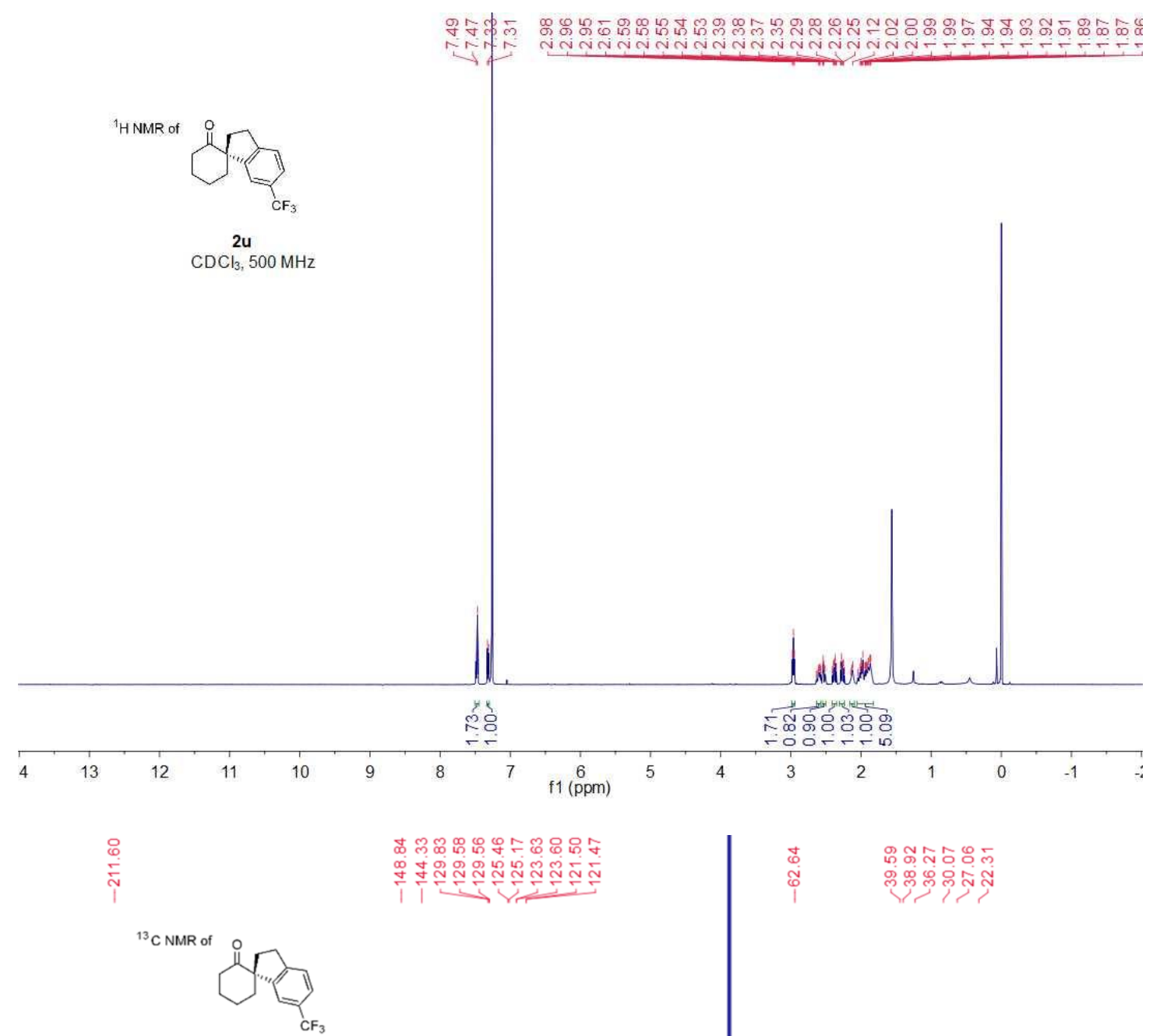

$\stackrel{2 \mathbf{u}}{\mathrm{CDCl}_{3}, 126 \mathrm{MHz}}$

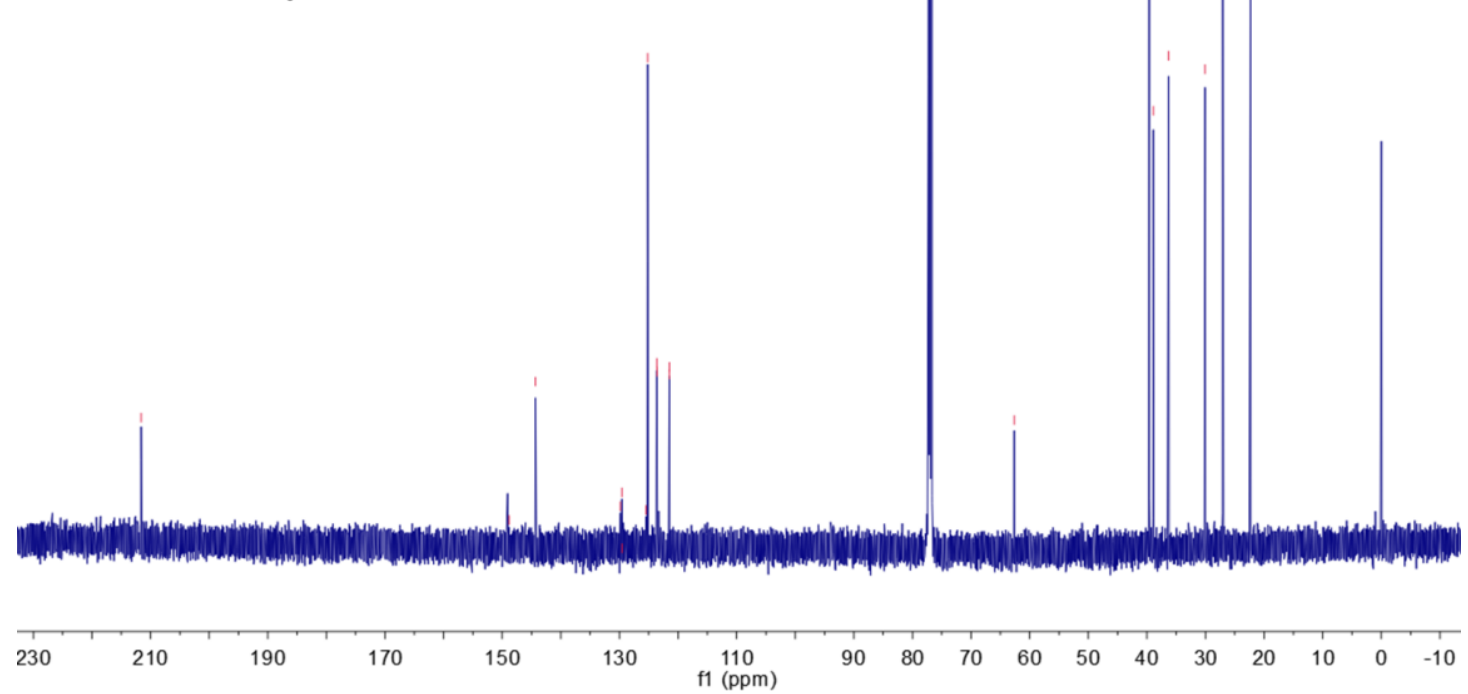




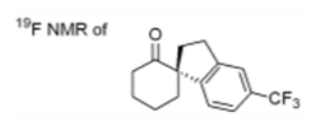

$2 \mathrm{u}$

$\mathrm{CDCl}_{3}, 376 \mathrm{MHz}$

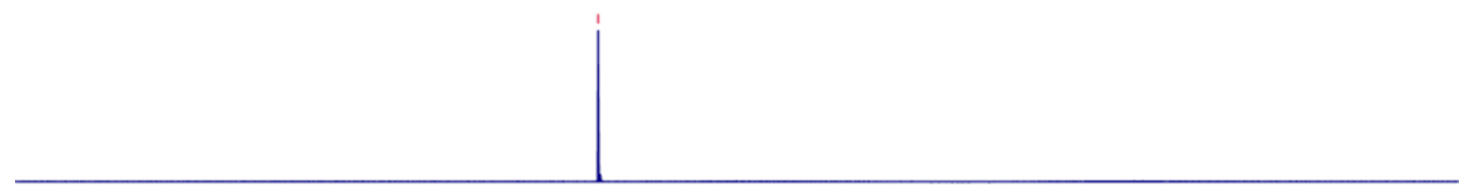

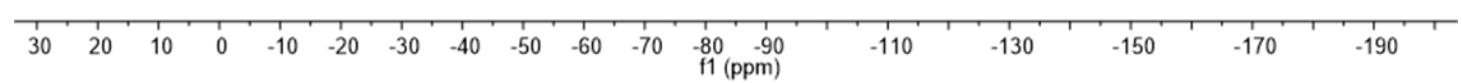

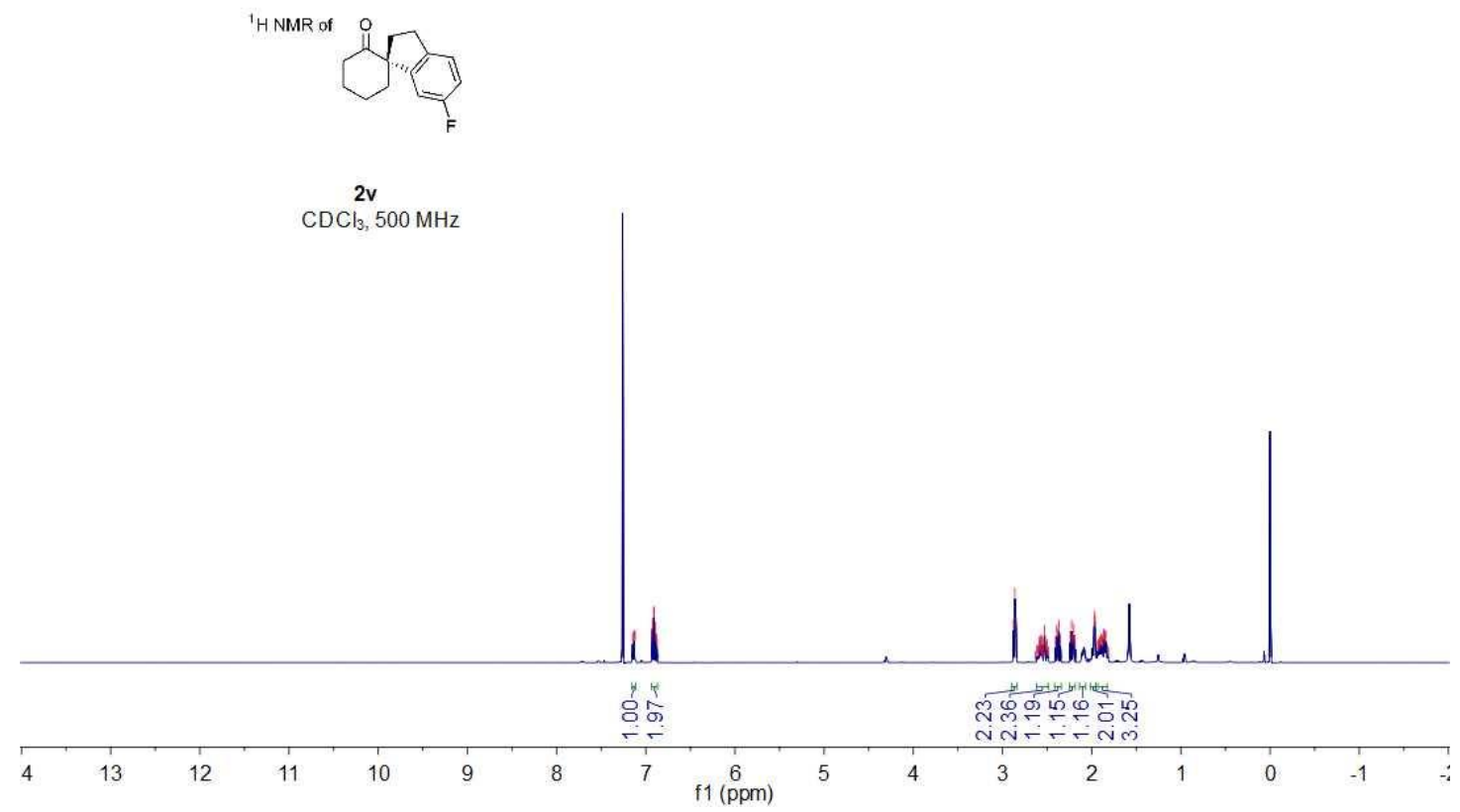



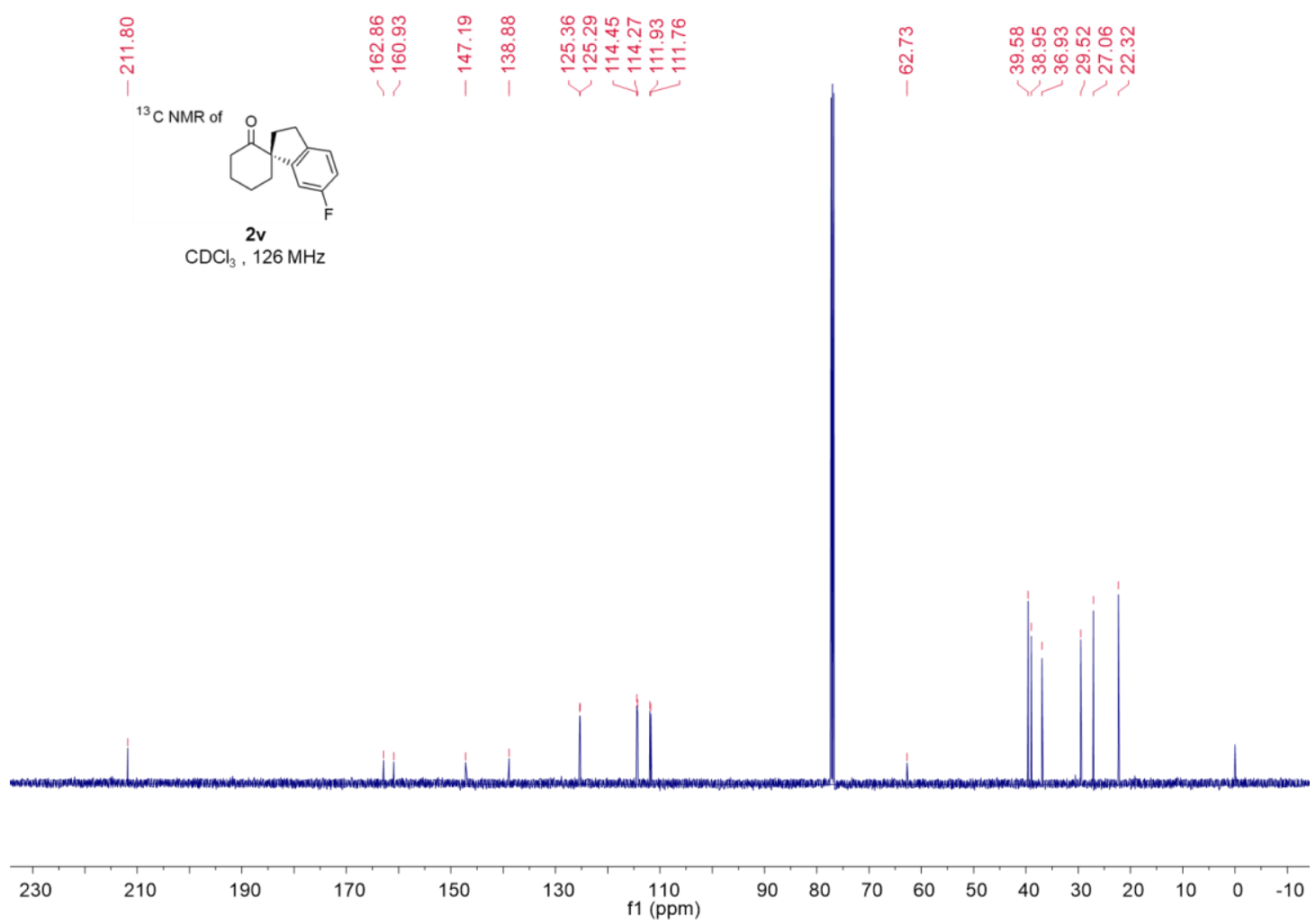

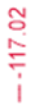

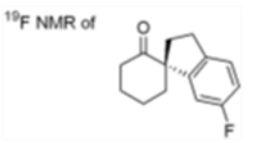

2v

$\mathrm{CDCl}_{3}, 376 \mathrm{MHz}$

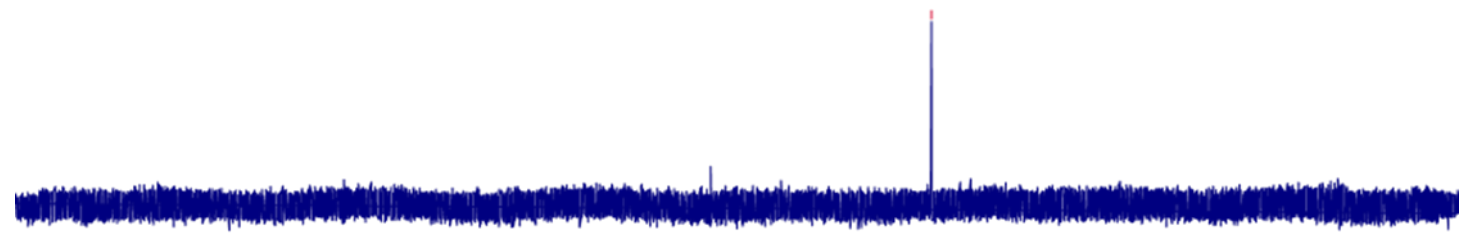

\begin{tabular}{lllllllllllllllllllll}
\hline & 20 & 10 & 0 & -10 & -20 & -30 & -40 & -50 & -60 & -70 & -80 & -90 & -110 & -130 & -150 & -170 & -190
\end{tabular} 
${ }^{1}$ H NMR of<smiles>O=C1CCCCC12CCc1cc(Cl)ccc12</smiles>

$\mathrm{CDCl}_{3}, 500 \mathrm{MHz}$

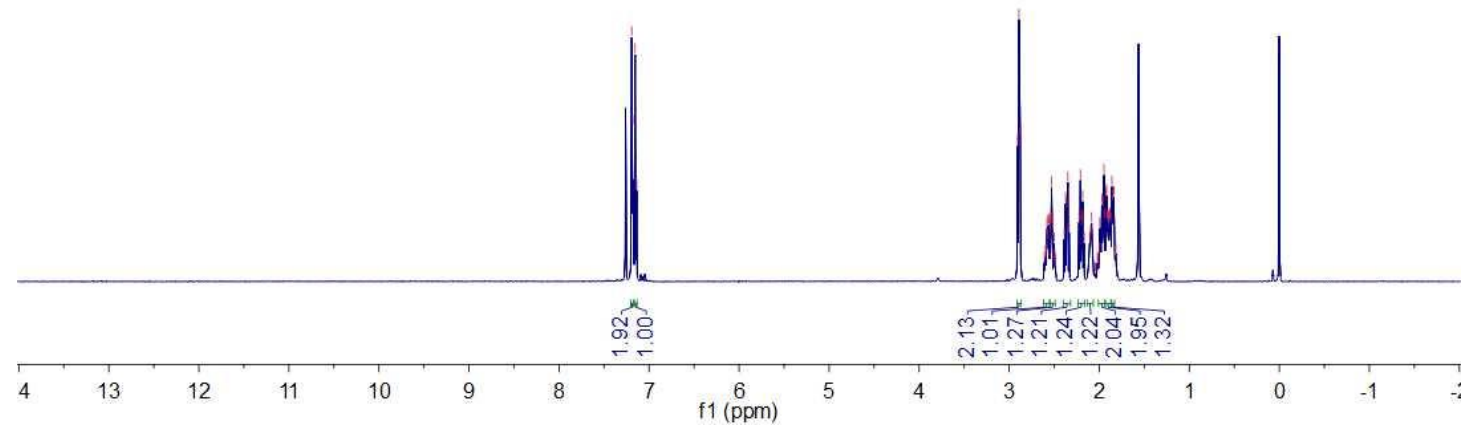

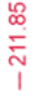

늉 윰ํㅠ

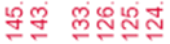

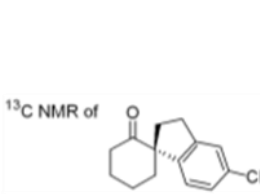

$2 w$

$\mathrm{CDCl}_{3}, 126 \mathrm{MHz}$

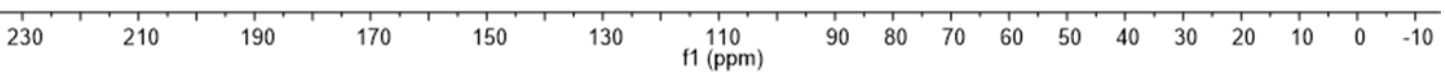


${ }^{1} \mathrm{HNMR}$ of

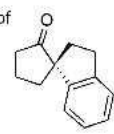

$2 x$

$\mathrm{CDCl}_{3}, 500 \mathrm{MHz}$

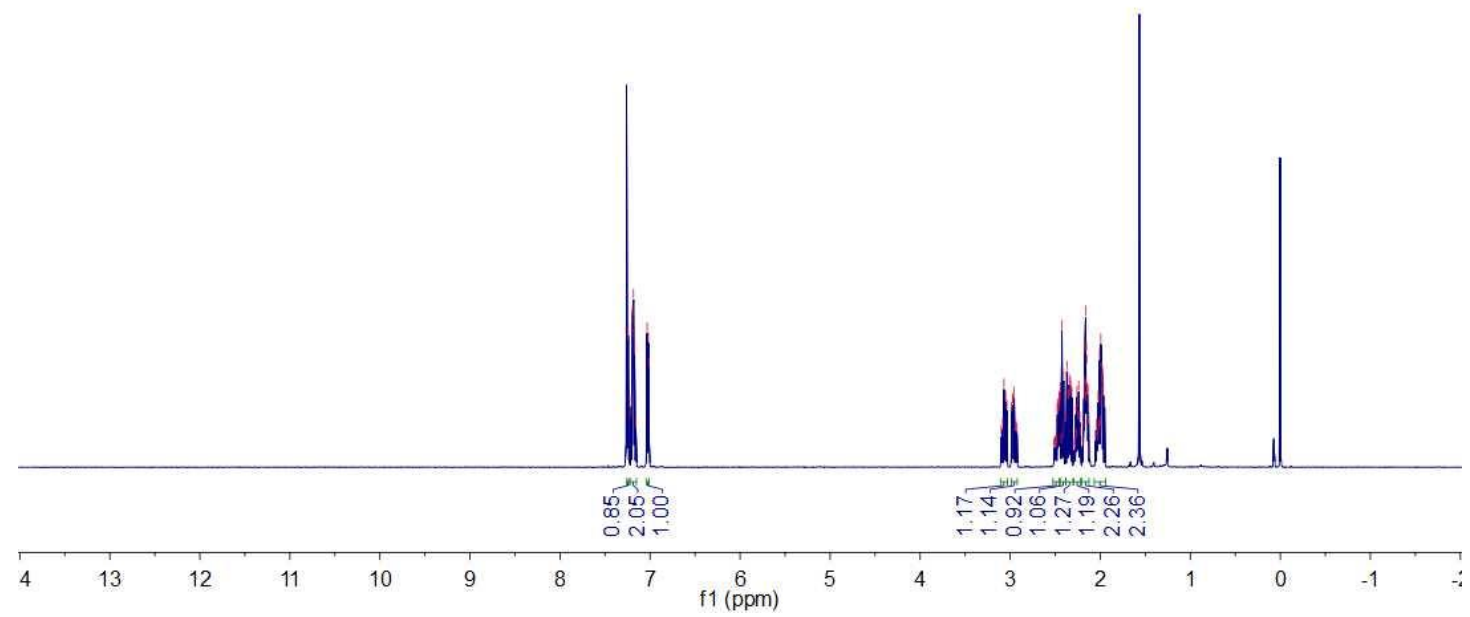

$\underset{\sim}{\infty}$

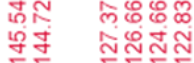

西

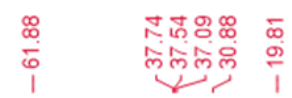

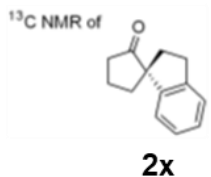

$\mathrm{CDCl}_{3}, 126 \mathrm{MHz}$

230

190

170

150

130 $\mathrm{f1}(\mathrm{ppm})$

$\begin{array}{lllllllllll}90 & 80 & 70 & 60 & 50 & 40 & 30 & 20 & 10 & 0 & -10\end{array}$ 
'H NMR of<smiles>COc1cccc2c1C1(C)C(=O)CCCC1C2</smiles>

$2 y$

$\mathrm{CDCl}_{3}, 500 \mathrm{MHz}$

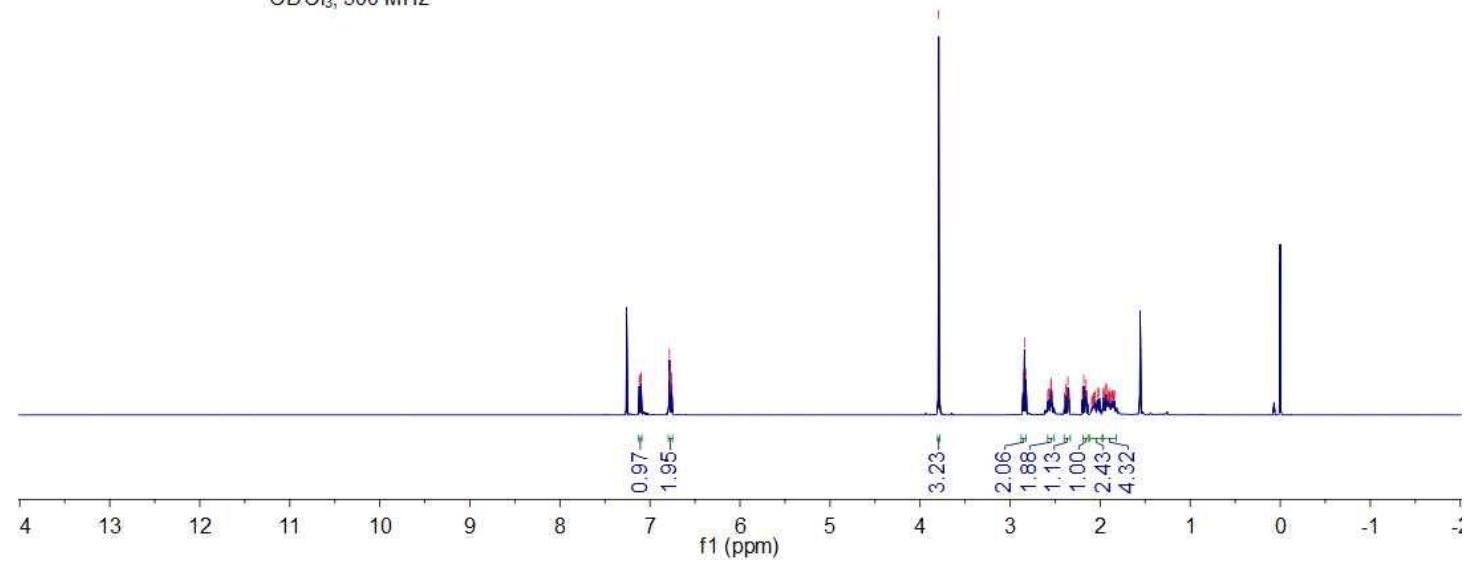

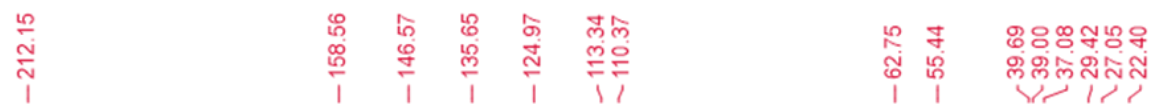

${ }^{13} \mathrm{CNMR}$ of

$2 y$

$\mathrm{CDCl}_{3}, 126 \mathrm{MHz}$

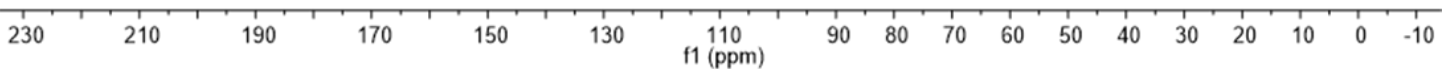


${ }^{1} \mathrm{H}$ NMR of<smiles>COc1ccc2c(c1)CCCC2=O</smiles>

$2 z$ $\mathrm{CDCl}_{3}, 500 \mathrm{MHz}$
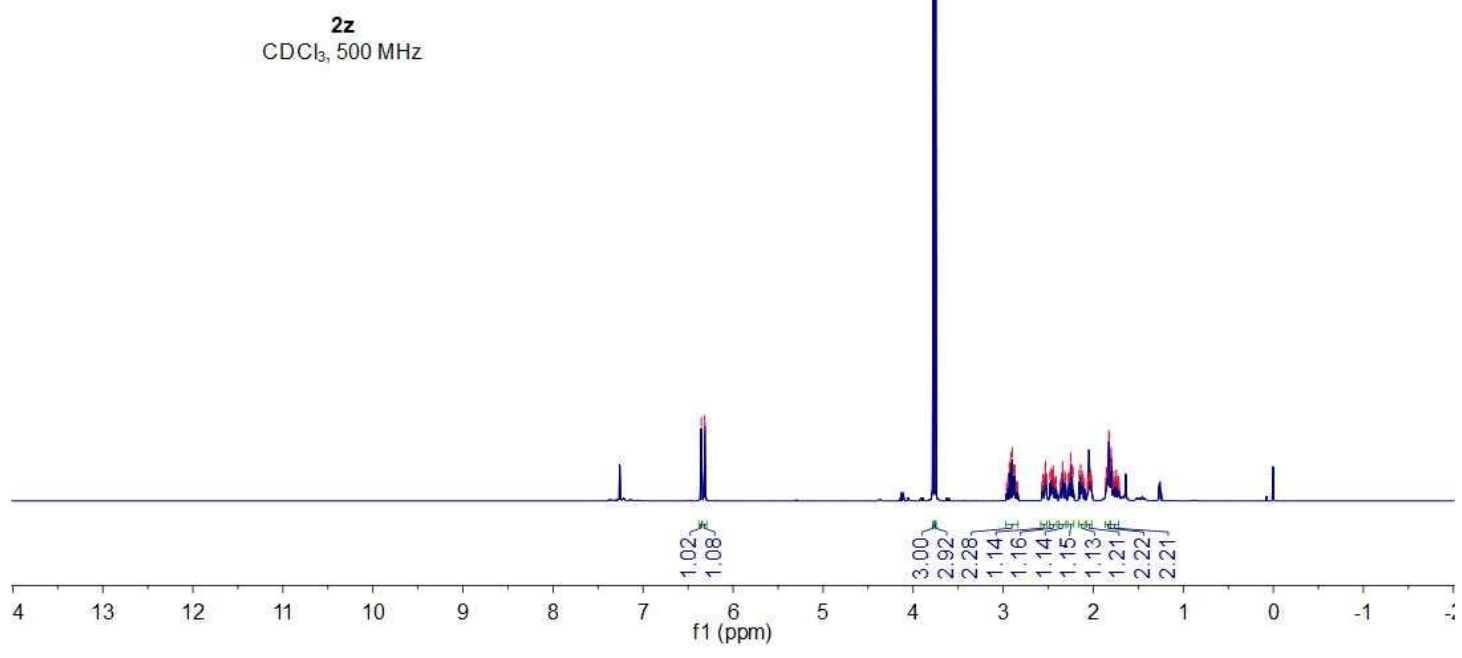

\begin{tabular}{|c|c|c|c|c|c|c|}
\hline$\frac{\infty}{\stackrel{N}{N}}$ & 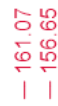 & 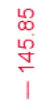 & $\begin{array}{l}\text { \% } \\
\stackrel{\text { Lj }}{\sim} \\
i\end{array}$ & $\begin{array}{l}9 \\
01 \\
80 \\
\circ 5 \\
11\end{array}$ & 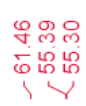 & 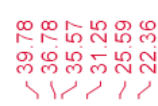 \\
\hline
\end{tabular}
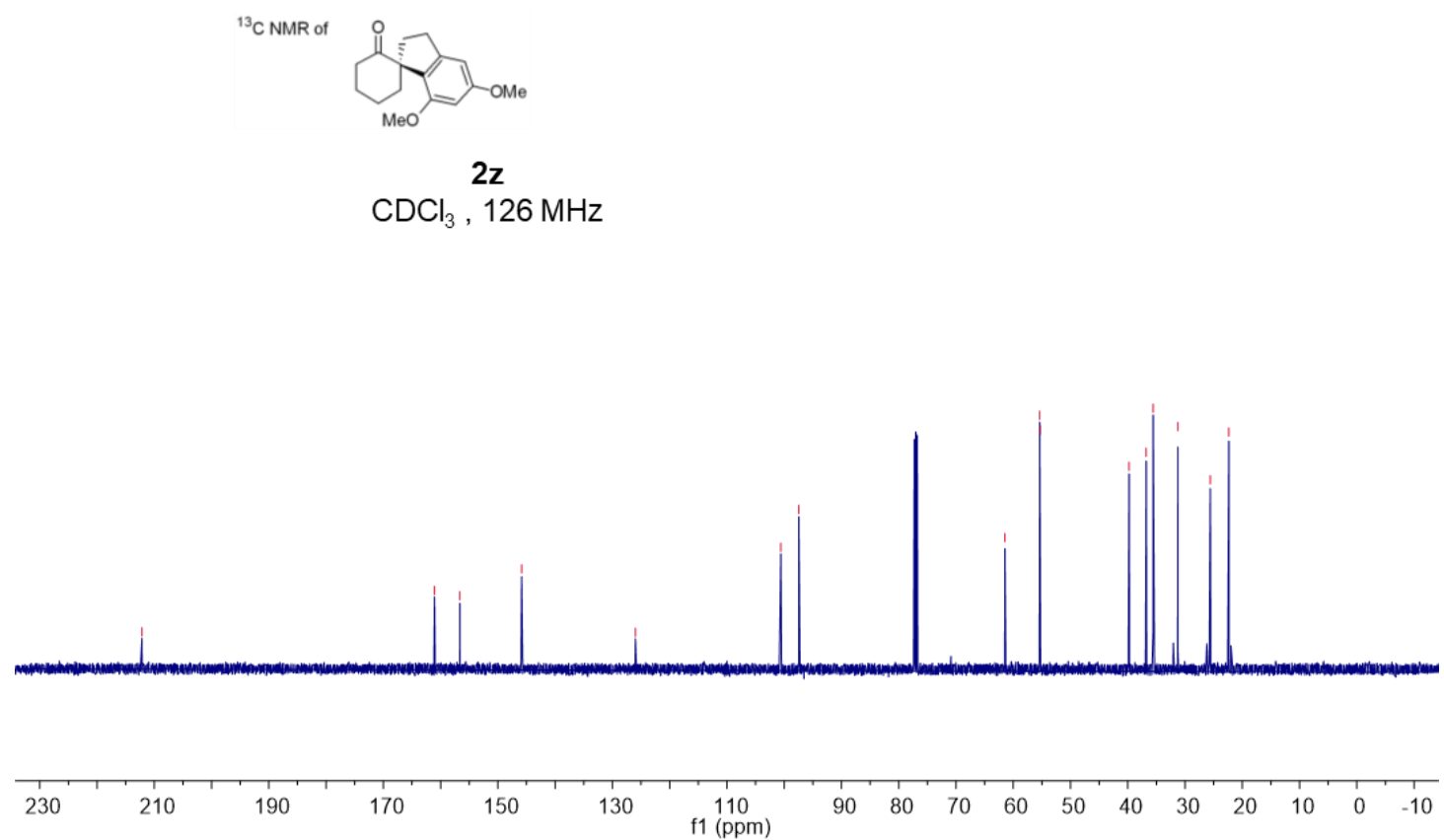
${ }^{1}$ HNMR of

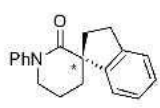

$\mathrm{CDCl}_{3}, 500 \mathrm{MHz}$

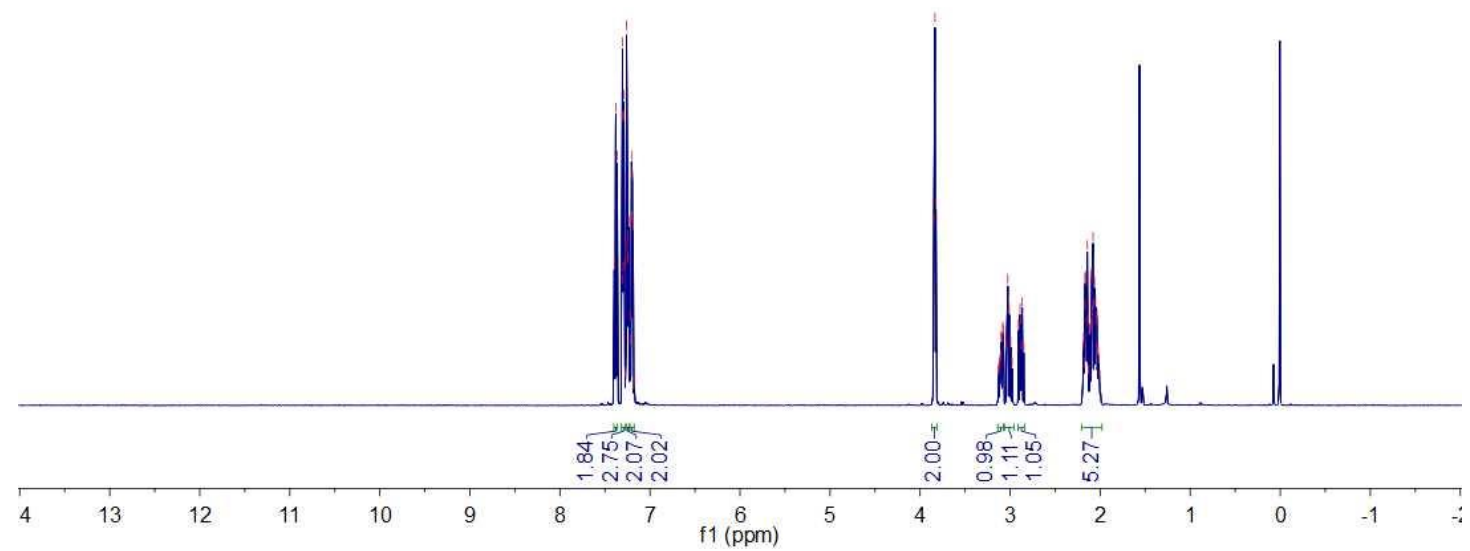

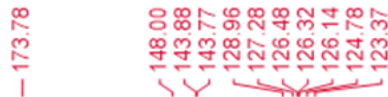

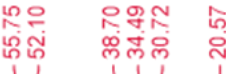

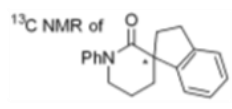

2aa

$\mathrm{CDCl}_{3}, 126 \mathrm{MHz}$
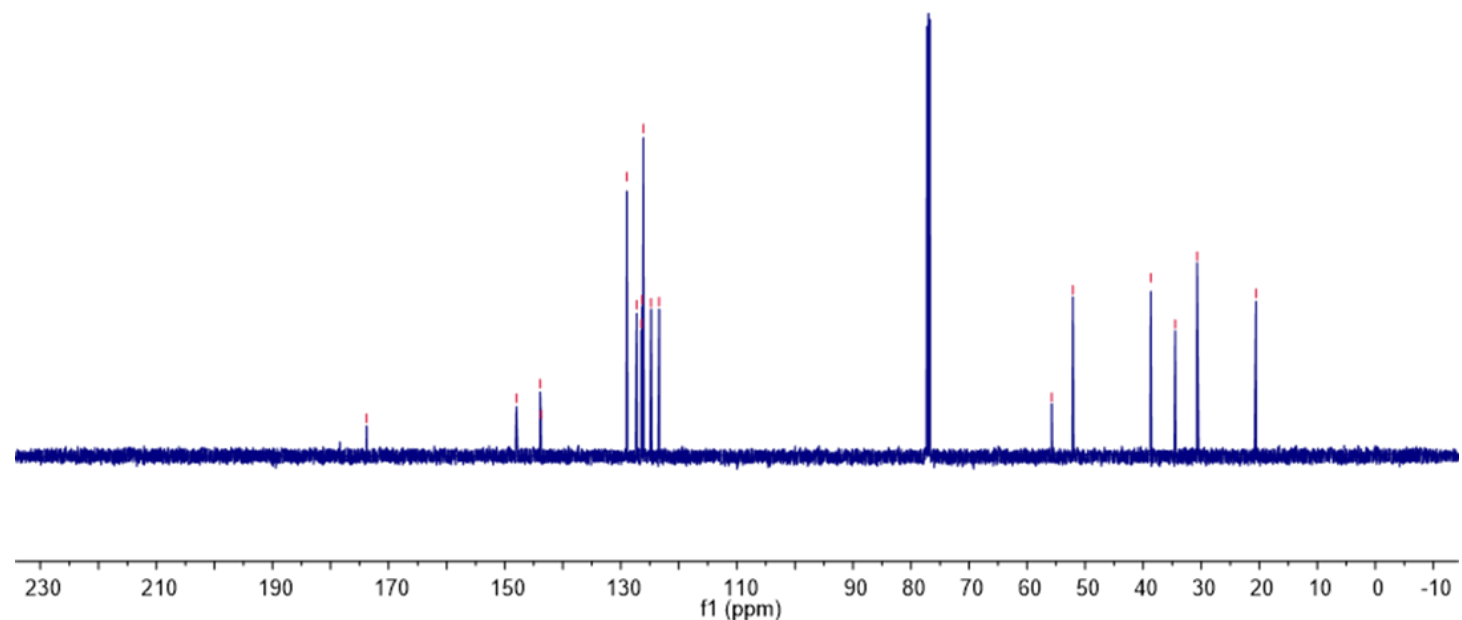
'HNMR of

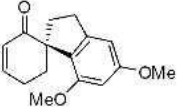

$\mathrm{CDCl}_{3}, 500 \mathrm{MHz}$

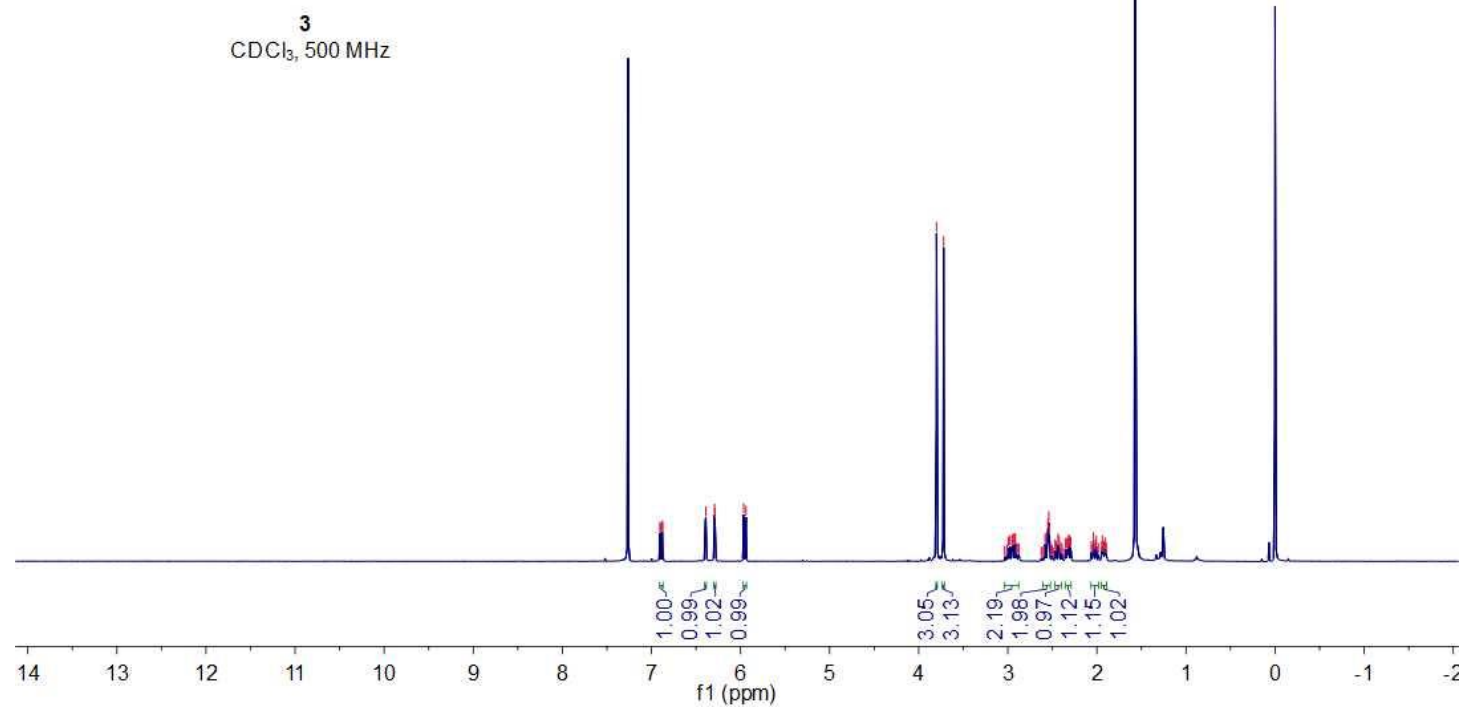

焉

${ }^{13} \mathrm{C} \mathrm{NMR}$ of

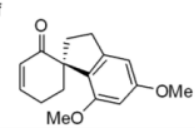

$\mathrm{CDCl}_{3}, 126 \mathrm{MHz}$

$\begin{array}{llllllllllllllllllllll}230 & 210 & 190 & 170 & 150 & 130 & 110 & 90 & 80 & 70 & 60 & 50 & 40 & 30 & 20 & 10 & 0 & -10\end{array}$




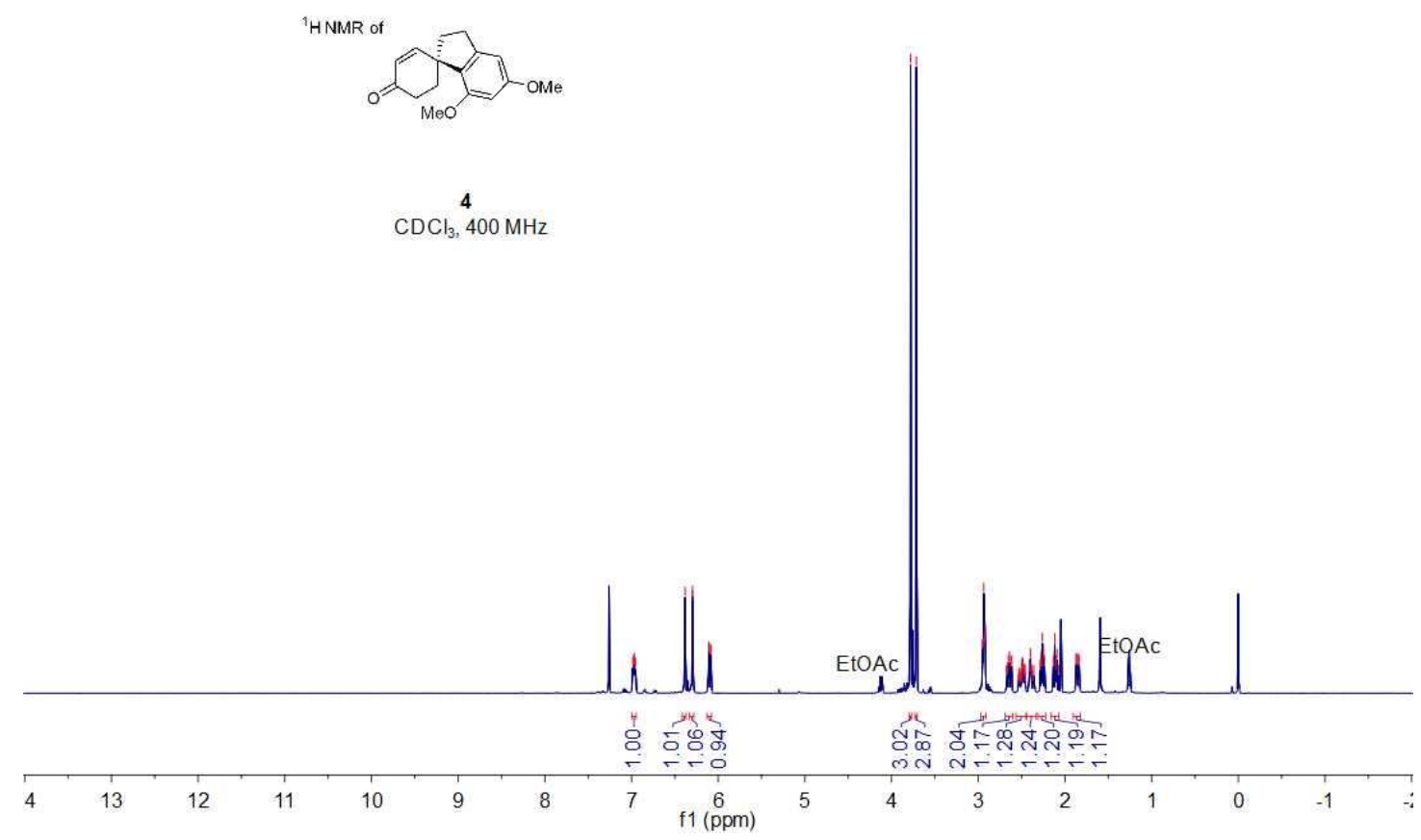

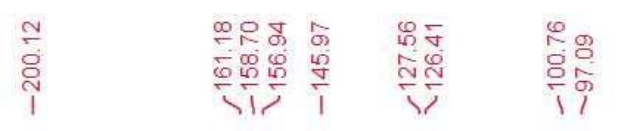

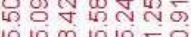

レ้

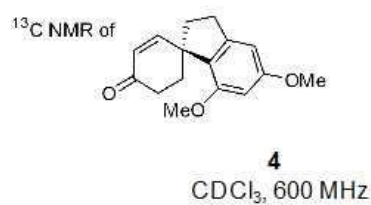

$\mathrm{CDCl}_{3}, 600 \mathrm{MHz}$

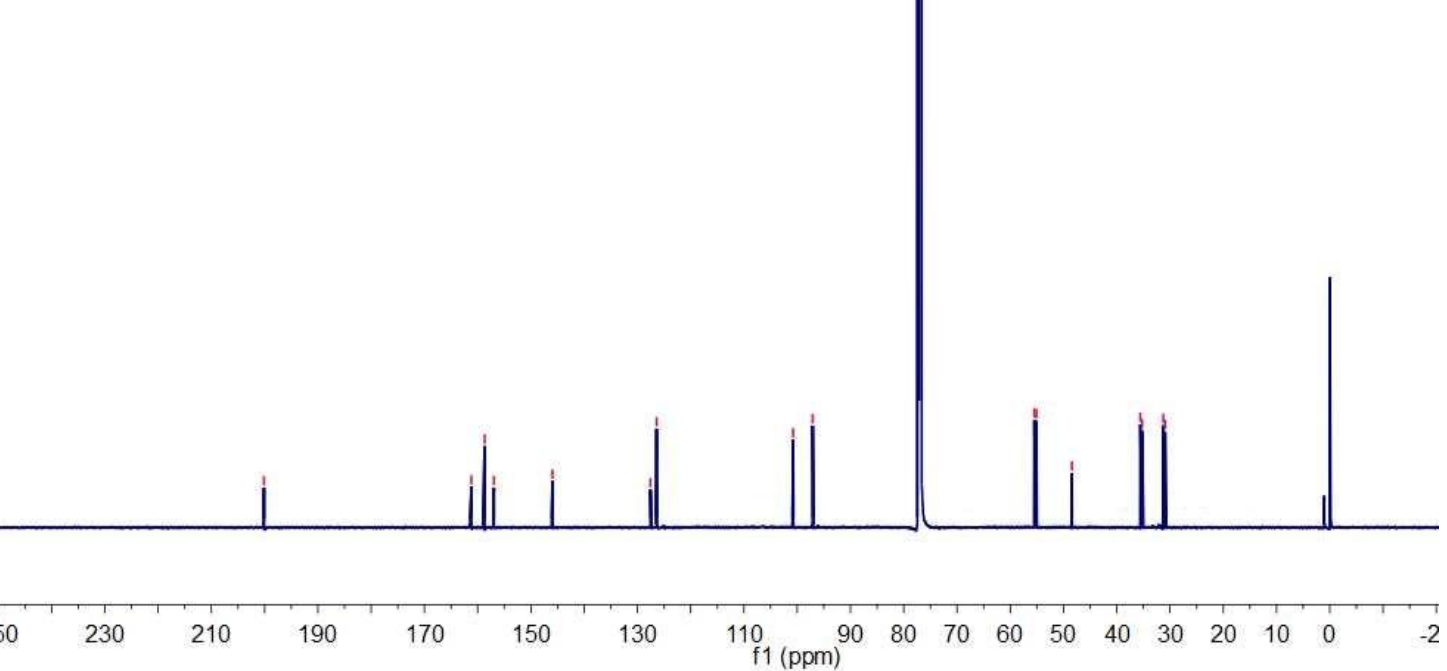




\section{X-ray information of 2a}

CD 1993958 contain the supplementary crystallographic data for this paper. These data can be obtained free of charge via www.ccdc.cam.ac.uk/conts/retrieving.html (or from the Cambridge Crystallographic Data Centre, 12 Union Road, Cambridge CB21EZ, UK; fax: (+44)1223-336-033; or deposit@ccdc.cam.ac.uk).

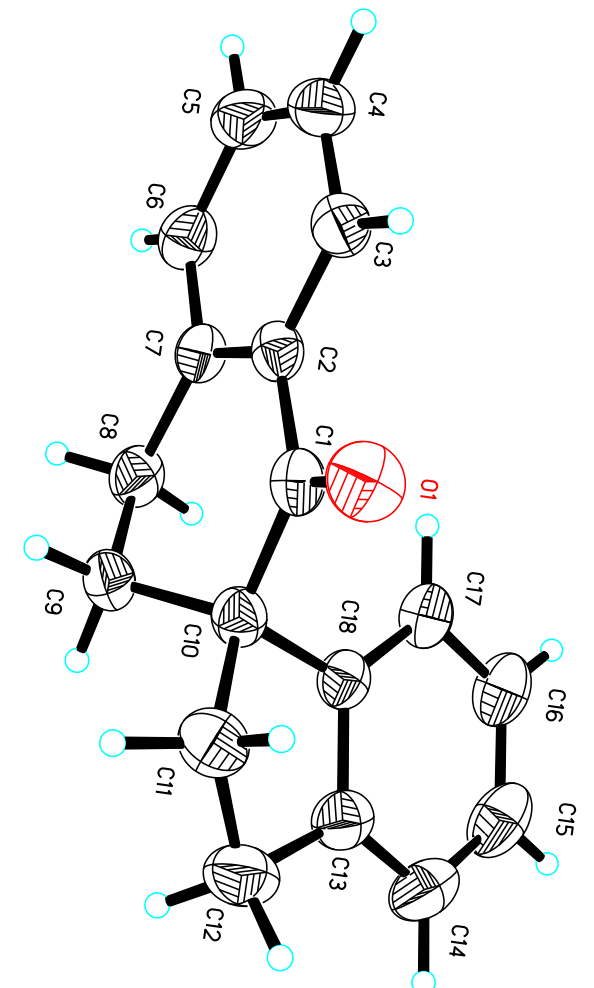

Figure 1. X-ray structure of $\mathbf{2 a}$ (Thermal ellipsoids were drawn at the $50 \%$ probability levels)

The crystals of $\mathbf{2 a}$ were obtained by dissolving $\mathbf{2 a}$ in tetrahydrofuran followed by slow evaporation of tetrahydrofuran.

Table 1. Crystal data and structure refinement for cu_d8v191038_0m.

Identification code cu_d8v191038_0m

Empirical formula C18 H16 O

Formula weight 248.31 
Temperature

Wavelength

Crystal system

Space group

Unit cell dimensions

Volume

Z

Density (calculated)

Absorption coefficient

$\mathrm{F}(000)$

Crystal size

Theta range for data collection

Index ranges

Reflections collected

Independent reflections

Completeness to theta $=67.679^{\circ}$

Absorption correction

Max. and min. transmission

Refinement method

Data / restraints / parameters

Goodness-of-fit on $\mathrm{F}^{2}$

Final R indices [I $>2 \operatorname{sigma}(\mathrm{I})]$

$\mathrm{R}$ indices (all data)

Absolute structure parameter
293(2) K

$1.54178 \AA$

Monoclinic

P 21

$\mathrm{a}=6.69450(10) \AA \quad \alpha=90^{\circ}$.

$\mathrm{b}=7.7519(2) \AA \quad \beta=101.4110(10)^{\circ}$.

$c=13.1155(3) \AA \quad \gamma=90^{\circ}$.

$667.18(3) \AA^{3}$

2

$1.236 \mathrm{Mg} / \mathrm{m}^{3}$

$0.579 \mathrm{~mm}^{-1}$

264

$0.180 \times 0.150 \times 0.130 \mathrm{~mm}^{3}$

8.167 to $67.490^{\circ}$.

$-8<=\mathrm{h}<=8,-9<=\mathrm{k}<=9,-15<=\mathrm{l}<=15$

9471

$2330[\mathrm{R}(\mathrm{int})=0.0317]$

$96.4 \%$

Semi-empirical from equivalents

0.7533 and 0.6151

Full-matrix least-squares on $\mathrm{F}^{2}$

$2330 / 1 / 173$

1.074

$\mathrm{R} 1=0.0349, \mathrm{wR} 2=0.0953$

$\mathrm{R} 1=0.0356, \mathrm{wR} 2=0.0962$

$0.15(11)$ 


\section{Extinction coefficient}

Largest diff. peak and hole
$0.23(3)$

0.100 and -0.098 e. $\AA^{-3}$ 